\title{
Symplectic reflection algebras, Calogero-Moser space, and deformed Harish-Chandra homomorphism
}

\author{
Pavel Etingof and Victor GinzBurg
}

\begin{abstract}
To any finite group $\Gamma \subset S p(V)$ of automorphisms of a symplectic vector space $V$ we associate a new multi-parameter deformation, $\mathrm{H}_{\kappa}$, of the algebra $\mathbb{C}[V] \# \Gamma$, smash product of $\Gamma$ with the polynomial algebra on $V$. The parameter $\kappa$ runs over points of $\mathbb{P}^{r}$, where $r=$ number of conjugacy classes of symplectic reflections in $\Gamma$. The algebra $\mathrm{H}_{\kappa}$, called a symplectic reflection algebra, is related to the coordinate ring of a Poisson deformation of the quotient singularity $V / \Gamma$. This leads to a symplectic analogue of McKay correspondence, which is most complete in case of wreath-products. If $\Gamma$ is the Weyl group of a root system in a vector space $\mathfrak{h}$ and $V=\mathfrak{h} \oplus \mathfrak{h}^{*}$, then the algebras $\mathbf{H}_{\kappa}$ are certain 'rational' degenerations of the double affine Hecke algebra introduced earlier by Cherednik.

Let $\Gamma=S_{n}$, the Weyl group of $\mathfrak{g}=\mathfrak{g l}_{n}$. We construct a 1-parameter deformation of the Harish-Chandra homomorphism from $\mathcal{D}(\mathfrak{g})^{\mathfrak{g}}$, the algebra of invariant polynomial differential operators on $\mathfrak{g l}_{n}$, to the algebra of $S_{n}$-invariant differential operators with rational coefficients on the space $\mathbb{C}^{n}$ of diagonal matrices. The second order Laplacian on $\mathfrak{g}$ goes, under the deformed homomorphism, to the Calogero-Moser differential operator on $\mathbb{C}^{n}$, with rational potential. Our crucial idea is to reinterpret the deformed Harish-Chandra homomorphism as a homomorphism: $\mathcal{D}(\mathfrak{g})^{\mathfrak{g}} \rightarrow$ spherical subalgebra in $\mathrm{H}_{\kappa}$, where $\mathrm{H}_{\kappa}$ is the symplectic reflection algebra associated to the group $\Gamma=S_{n}$. This way, the deformed Harish-Chandra homomorphism becomes nothing but a description of the spherical subalgebra in terms of 'quantum' Hamiltonian reduction.

In the 'classical' limit $\kappa \rightarrow \infty$, our construction gives an isomorphism between the spherical subalgebra in $\mathrm{H}_{\infty}$ and the coordinate ring of the Calogero-Moser space. We prove that all simple $\mathrm{H}_{\infty}$-modules have dimension $n$ !, and are parametrised by points of the Calogero-Moser space. The family of these modules forms a distinguished vector bundle on the CalogeroMoser space, whose fibers carry the regular representation of $S_{n}$. Moreover, we prove that the algebra $\mathrm{H}_{\infty}$ is isomorphic to the endomorphism algebra of that vector bundle.
\end{abstract}

1. Introduction

\section{Table of Contents}

PART 1. Symplectic reflection algebras

2. Koszul patterns and deformations

3. Representation theory of the algebra $\mathrm{H}_{0, c}$

4. The rational Cherednik algebra

5. Automorphisms and derivations of the algebra $\mathrm{H}_{\kappa}$

PART 2. Harish-Chandra homomorphism

6. The radial part construction

7. Deformation of the Harish-Chandra homomorphism

8. Example: $\mathfrak{g}=\mathfrak{s l}_{2}$

9. The kernel of the Harish-Chandra homomorphism

10. Proof of Theorem 7.3

11. Calogero-Moser space for wreath-products

APPENDICES.

12. Appendix A: Almost commuting matrices

13. Appendix B: Geometric construction of $V_{k}$

14. Appendix C: Small representations

15. Appendix D: Deformations, Poisson brackets and cohomology

16. Appendix E: Some examples

17. Appendix F: Open questions

18. Appendix G: Results of Kostant and shift operators 


\section{Introduction}

Given an action of a finite group $\Gamma$ on a smooth affine algebraic variety $X$, one can study the algebraic structure of the orbifold $X / \Gamma$ through its coordinate ring $\mathbb{C}[X]^{\Gamma}$, the ring of $\Gamma$-invariant regular functions on $X$. It is known, however, that if the $\Gamma$-action on $X$ is not free the (possibly singular) space $X / \Gamma$ does not necessarily reflect the features of the $\Gamma$ action quite adequately. For instance, one is led to introduce various "stringy" topological invariants, cf. [Ba], which do not coincide with the ordinary topological invariants of $X / \Gamma$, but are rather related to certain "resolutions" of $X / \Gamma$. A standard approach to this problem, see e.g. $[\mathrm{Co}]$, is to replace the commutative coordinate ring $\mathbb{C}[X]^{\Gamma}$ by the smash-product algebra $\mathbb{C}[X] \# \Gamma$. The two algebras are Morita equivalent, hence lead to equivalent theories, provided the $\Gamma$-action on $X$ is free, but are not equivalent in general. Although the algebra $\mathbb{C}[X] \# \Gamma$ is non-commutative, it is believed that the "right" geometry of the $\Gamma$-action on $X$ can be read off from the "non-commutative algebraic geometry" of $\mathbb{C}[X] \# \Gamma$. This is the approach that we adopt in the present paper.

Specifically, we will study deformations of some interesting orbifolds $X / \Gamma$ through non-commutative deformations of the corresponding algebra $\mathbb{C}[X] \# \Gamma$. We will see that a systematic use of this ideology leads, via Morita equivalence, to various new results in conventional "commutative" algebraic geometry which have been unaccessible by purely "commutative" methods.

Another fascinating feature of our approach is that genuine deformations of $X / \Gamma$ coming from "commutative" algebraic varieties stand on an equal footing with purely "quantum" deformations that are not associated with any algebraic varieties whatsoever. "Commutative" and "quantum" deformations only differ by the values of deformation parameters. Thus, "quantum" deformations may be viewed as quantizations of "commutative" ones, and Kontsevich's quantization plays a role here. On the other hand, the algebras arising in such "quantum" deformations are closely related to the algebras of invariant polynomial differential operators on semisimple Lie algebras and Quiver varieties. Thus, on the "quantum" side, we enter the realm of representation theory, which was our original motivation.

Symplectic reflection algebras. Let $V$ be a finite dimensional vector space over $\mathbb{C}$, and $T V$ its tensor algebra. Let $\Gamma \subset \mathrm{GL}(V)$ be a finite group, and $(T V) \# \Gamma$ the smashproduct of $T V$ with $\mathbb{C} \Gamma$, the group algebra of $\Gamma$. Given a skew-symmetric $\mathbb{C}$-bilinear pairing $\kappa: V \times V \rightarrow \mathbb{C} \Gamma$, put

$$
\mathrm{H}_{\kappa}:=(T V \# \Gamma) / I\left\langle x \otimes y-y \otimes x-\kappa(x, y) \in T^{2} V \oplus \mathbb{C} \Gamma\right\rangle_{x, y \in V},
$$

where $I\langle\ldots\rangle$ stands for the two-sided ideal in $(T V) \# \Gamma$ generated by the indicated set. Thus, $\mathrm{H}_{\kappa}$ is an associative algebra.

In the special case $\kappa=0$, the situation simplifies drastically since all the generators $x \in V$ commute with each other. The resulting algebra is therefore isomorphic to the smash-product algebra: $\mathrm{H}_{\kappa=0}=(S V) \# \Gamma$. Note that this algebra has a natural grading obtained by placing $\mathbb{C} \Gamma$ in grade degree zero, and $V$ in grade degree 1 . 
In general, if $\kappa \neq 0$, the defining relations, see (1.1), become inhomogeneous, hence assigning $\mathbb{C} \Gamma$ grade degree zero, and $V$ grade degree 1 , one only gets an increasing filtration $F_{\bullet}\left(\mathrm{H}_{\kappa}\right)$ on the corresponding algebra $\mathrm{H}_{\kappa}$, such that, for any $i, j \geq 0$, we have: $F_{i}\left(\mathrm{H}_{\kappa}\right) \cdot F_{j}\left(\mathrm{H}_{\kappa}\right) \subset F_{i+j}\left(\mathrm{H}_{\kappa}\right)$. Let $\operatorname{gr}\left(\mathrm{H}_{\kappa}\right)=\bigoplus_{i} F_{i}\left(\mathrm{H}_{\kappa}\right) / F_{i-1}\left(\mathrm{H}_{\kappa}\right)$ denote the associated graded algebra. It is immediate from (1.1) that, for any $x, y \in V \subset \operatorname{gr}\left(\mathrm{H}_{\kappa}\right)$, in $\operatorname{gr}\left(\mathrm{H}_{\kappa}\right)$ we have: $x y-y x=0$. Therefore the tautological imbedding: $V \hookrightarrow \operatorname{gr}\left(\mathrm{H}_{\kappa}\right)$ extends to a well-defined and surjective graded algebra homomorphism: $\mathrm{H}_{\kappa=0}=(S V) \# \Gamma \longrightarrow \operatorname{gr}\left(\mathrm{H}_{\kappa}\right)$. We say that Poincaré-Birkhoff-Witt (PBW-)property holds for $\mathrm{H}_{\kappa}$ if this morphism is an isomorphism.

From now on we assume, in addition, that $V$ is a symplectic vector space with symplectic 2-form $\omega$, and that $\Gamma \subset S p(V)$. The triple $(V, \omega, \Gamma)$ is said to be indecomposable if there is no $\omega$-orthogonal direct sum decomposition: $V=V_{1} \oplus V_{2}$, where $V_{i}$ are $\Gamma$-stable proper symplectic vector subspaces in $V$.

Definition 1.2 An element $s \in \Gamma$ is called a symplectic reflection if $\operatorname{rk}(\operatorname{Id}-s)=2$.

Let $S$ denote the set of symplectic reflections in $\Gamma$. The group $\Gamma$ acts on $S$ by conjugation. For each $s \in S$, write $\omega_{s}$ for the (possibly degenerate) skew-symmetric form on $V$ which coincides with $\omega$ on $\operatorname{Im}(\operatorname{Id}-s)$, and has $\operatorname{Ker}(\operatorname{Id}-s)$ as the radical (recall that there is an $\omega$-orthogonal direct sum decomposition $V=\operatorname{Im}(\operatorname{Id}-s) \oplus \operatorname{Ker}(\operatorname{Id}-s)$ ).

Theorem 1.3 Assume that $(V, \omega, \Gamma)$ is an indecomposable triple. Then, $P B W$-property holds for $\mathrm{H}_{\kappa}$ if and only if there exists a constant $t \in \mathbb{C}$, and an $\mathrm{Ad} \Gamma$-invariant function $c: S \rightarrow \mathbb{C}, s \mapsto c_{s}$, such that the pairing $\kappa$ has the form:

$$
\kappa(x, y)=t \cdot \omega(x, y) \cdot 1+\sum_{s \in S} c_{s} \cdot \omega_{s}(x, y) \cdot s \quad, \quad \forall x, y \in V .
$$

Remarks (i) Our proof of the Theorem shows that the algebra $\mathrm{H}_{\kappa}$ associated to a pairing $\kappa$ of the type described by the formula above always satisfies the PBW-property, even if the triple $(V, \omega, \Gamma)$ is decomposable.

(ii) An analogue of Theorem 1.3 holds in a more general setup, where $V$ is any (not necessarily symplectic) vector space, and $\Gamma \subset \mathrm{GL}(V)$ is a finite subgroup containing no elements $s \in \Gamma$ such that $\operatorname{rk}(\operatorname{Id}-s)=1$. The proof of Theorem 1.3 given in $\S 2$ shows that, in this generality, PBW-property holds for $\mathrm{H}_{\kappa}$ if and only if $\kappa(x, y)=$ $\omega(x, y) \cdot 1+\sum_{s \in S} \omega_{s}(x, y) \cdot s, \forall x, y \in V$, where $\omega$ is a (possibly degenerate) skew-symmetric $\Gamma$-invariant form on $V$, symplectic reflections are defined as in Definition 1.2 (involving no reference to any symplectic structure), and $\left\{\omega_{s}\right\}_{s \in S}$ stands for an arbitrary $\Gamma$-equivariant collection of skew-symmetric forms such that: $\operatorname{Ker}(\operatorname{Id}-s) \subset \operatorname{Radical}\left(\omega_{s}\right), \forall s \in S$.

Theorems 1.51 .7 below also hold in this generality. $\diamond$

Let $\mathrm{C}$ denote the vector space of Ad $\Gamma$-invariant functions on $S$. We have

$$
\operatorname{dim} C=\text { number of Ad } \Gamma \text {-conjugacy classes in } S .
$$


From now on, we assume that the pairing $\kappa$ in (1.1) has the form described in Theorem 1.3, for a certain $t \in \mathbb{C}$ and $c \in \mathrm{C}$. The corresponding algebra $\mathrm{H}_{t, c}:=\mathrm{H}_{\kappa}$ will be referred to as a symplectic reflection algebra.

Let $\mathbf{e}=\frac{1}{|\Gamma|} \sum_{g \in \Gamma} g \in \mathbb{C} \Gamma$, be the 'symmetriser' idempotent, viewed as an element of $\mathrm{H}_{t, c}$. The algebra $\mathbf{e H}_{t, c} \mathbf{e} \subset \mathrm{H}_{t, c}$ will be called the spherical subalgebra in $\mathrm{H}_{t, c}$. For $t=0$ and $c=0$, we have: $\mathbf{e H}_{0,0} \mathbf{e}=\mathbf{e}(S V \# \Gamma) \mathbf{e} \simeq(S V)^{\Gamma}$, the algebra of $\Gamma$-invariants. Furthermore, for any $(t, c) \in \mathbb{C} \oplus \mathrm{C}$, Theorem 1.3 yields: $\operatorname{gr}\left(\mathbf{e H}_{t, c} \mathbf{e}\right) \simeq(S V)^{\Gamma}$, see (2.4).

The space $\mathrm{H}_{t, c} \mathbf{e}$ has an obvious left $\mathrm{H}_{t, c}$-module structure, and right $\mathbf{e} \mathrm{H}_{t, c} \mathbf{e}$-module structure. Given an algebra $A$, and a left, resp. right, $A$-module $M$, we set: $M^{\vee}=$ $\operatorname{Hom}_{A \text {-left }}(M, A)$, resp. ${ }^{\vee} M=\operatorname{Hom}_{A \text {-right }}(M, A)$.

Theorem 1.5 (i) $\mathbf{e H}_{t, c} \mathbf{e}$ is a finitely generated Gorenstein algebra without zero divisors.

(ii) $\mathrm{H}_{t, c} \mathbf{e}$ is a finitely generated, Cohen-Macaulay right $\mathbf{e H}_{t, c} \mathbf{e}$-module.

(iii) We have: ${ }^{\vee}\left(\mathrm{H}_{t, c} \mathbf{e}\right)=\mathbf{e H}_{t, c}$, and $\left(\mathbf{e H}_{t, c}\right)^{\vee}=\mathrm{H}_{t, c} \mathbf{e}$. In particular, $\mathrm{H}_{t, c} \mathbf{e}$ is reflexive.

(iv) The left $\mathrm{H}_{t, c}$-action induces an algebra isomorphism: $\mathrm{H}_{t, c} \stackrel{\sim}{\longrightarrow} \operatorname{End}_{\mathbf{e H}_{t, c} \mathbf{e}}\left(\mathrm{H}_{t, c} \mathbf{e}\right)$.

Remark. For a definition of a (not necessarily commutative) Gorenstein ring, resp. Cohen-Macaulay module, which, in the commutative case reduces to the standard definition as given, e.g. in $[\mathrm{BBG}]$, the reader is referred to $\S 3$ (proof of Thm. 1.5).

For any $t \neq 0$, let $\mathrm{A}_{t}=T V / I\langle x \cdot y-y \cdot x-t \cdot \omega(x, y)\rangle_{x, y \in V}$ be the Weyl algebra of the symplectic vector space $(V, t \cdot \omega)$; the algebras $\mathrm{A}_{t}$ are isomorphic to each other, for all $t \neq 0$. We observe that, for $c=0$ (and any $t \neq 0$ ), we have: $\mathrm{H}_{t, 0}=\mathrm{A}_{t} \# \Gamma$. We will show (see Theorem 2.16) that, for any fixed $t \neq 0$, the family $\left\{\mathrm{H}_{t, c}\right\}_{c \in C}$ gives a universal deformation of the algebra $A_{t} \# \Gamma$. Furthermore, the spherical subalgebra $\mathbf{e H}_{t, 0} \mathbf{e}$ is isomorphic to $A_{t}^{\Gamma}$, the subalgebra of $\Gamma$-invariants in the Weyl algebra, and (for any fixed $t \neq 0$ ) the family $\left\{\mathbf{e H}_{t, c} \mathbf{e}\right\}_{c \in C}$ gives a universal deformation of the algebra $A_{t}^{\Gamma}$.

Theorem 1.6 For any $c \in \mathrm{C}$, the algebra $\mathbf{e H}_{t, c} \mathbf{e}$ is commutative if and only if $t=0$.

Example: Kleinian singularity. Let $\Gamma \subset S L_{2}(\mathbb{C})$ be a finite subgroup acting on $V=\mathbb{C}^{2}$. The action on $V \backslash\{0\}$ is free, and any element of $\Gamma \backslash\{1\}$ is a symplectic reflection. This case has been studied by Crawley-Boevey and Holland $[\mathrm{CBH}]$.

Quasi-classical case: $\kappa=(\mathbf{0}, \mathbf{c})$. Assume next that $t=0$ and $c \neq 0$. The algebra $\mathrm{H}_{0, c}, c \neq 0$, has some very interesting special features making it quite different from

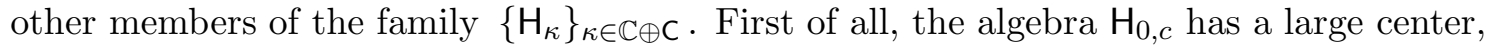
$\mathrm{Z}_{0, c}=\mathrm{Z}\left(\mathrm{H}_{0, c}\right)$, as opposed to the algebras $\mathrm{H}_{\kappa}$ for generic $\kappa$. In more detail, the standard filtration on $\mathrm{H}_{0, c}$ induces a filtration on $\mathrm{Z}\left(\mathrm{H}_{0, c}\right)$, and we will show that:

$$
\operatorname{gr} \mathrm{Z}\left(\mathrm{H}_{0, c}\right)=\mathrm{Z}\left(\mathrm{H}_{0,0}\right)=\mathrm{Z}(S V \# \Gamma)=(S V)^{\Gamma} \text {. }
$$

Further, for any $c \in \mathrm{C}$, we use the construction of Hayashi [Ha], to make both the spherical subalgebra $\mathbf{e H}_{0, c} \mathbf{e}$ and the center $\mathrm{Z}_{0, c}$ Poisson algebras, which reduce to the standard 
Poisson structure on $(S V)^{\Gamma}$, if $c=0$. Unlike the case $c=0$, the center of $\mathrm{H}_{0, c}$ is harder to describe explicitly. To this end, we generalize the classical Satake isomorphism for an affine Hecke algebra (as proposed by Lusztig [Lu]), and show that the map: $z \mapsto z \cdot \mathbf{e}$ establishes a Poisson algebra isomorphism: $\mathrm{Z}_{0, c} \stackrel{\sim}{\longrightarrow} \mathbf{e H}_{0, c} \mathbf{e}$, which we call the Satake isomorphism.

Let $\mathcal{R}$ denote the coherent sheaf on Spec $Z_{0, c}$ corresponding to $\mathrm{H}_{0, c} \mathbf{e}$, viewed as a (finitely generated) $Z_{0, c}$-module, that is: $\Gamma\left(\operatorname{Spec} Z_{0, c}, \mathcal{R}\right)=\mathrm{H}_{0, c} \mathbf{e}$. We will view points of Spec $Z_{0, c}$ as algebra homomorphisms $\chi: Z_{0, c} \longrightarrow \mathbb{C}$. Then, for any point $\chi \in \operatorname{Spec} Z_{0, c}$, the geometric fiber of the sheaf $\mathcal{R}$ at $\chi$ equals: $\mathrm{H}_{0, c} \mathbf{e} \bigotimes_{\mathrm{z}_{0, c}} \chi$. The left action of $\mathrm{H}_{0, c}$ on $\mathrm{H}_{0, c}$ e gives an $\mathrm{H}_{0, c}$-module structure on each geometric fiber of $\mathcal{R}$.

In the theorem below, we fix $U \subset$ Spec $Z_{0, c}$, a Zariski-open affine subset, and use the subscript ' $U$ ' to denote 'restriction to $U$ '. In particular we write $Z_{U}:=\mathbb{C}[U] \supset Z_{0, c}$, and $\mathrm{H}_{U}:=\mathrm{Z}_{U} \bigotimes_{\mathrm{Z}_{0, c}} \mathrm{H}_{0, c}$, and also $\mathcal{R}_{U}:=\left.\mathcal{R}\right|_{U}$.

Theorem 1.7 Let $U$ be an affine Zariski-open subset contained in the smooth locus of Spec $\mathrm{Z}_{0, c}$. Then we have:

(i) The sheaf $\mathcal{R}_{U}$ is locally free, and the algebra $\mathrm{H}_{U}$ is isomorphic to $\operatorname{End}_{\mathrm{z}_{U}}\left(\mathcal{R}_{U}\right)$.

(ii) The algebra $\mathrm{H}_{U}$ is Morita equivalent to $\mathbf{Z}_{U}$, i.e. the categories of finitely generated $\mathrm{H}_{U}$-modules and $\mathrm{Z}_{U}$-modules are equivalent.

(iii) Any simple $\mathrm{H}_{U}$-module is isomorphic to one of the form: $\mathrm{H}_{U} \mathbf{e} \bigotimes_{\mathrm{z}_{U}} \chi$, for a certain character $\chi: Z_{U} \rightarrow \mathbb{C}$. In particular, the action of the center defines a bijection between the isomorphism classes of simple $\mathrm{H}_{U}$-modules and points of $U$.

(iv) Any simple $\mathrm{H}_{U}$-module has dimension $|\Gamma|$ and is isomorphic, as a $\Gamma$-module, to the regular representation of $\Gamma$.

If Spec $\mathrm{Z}_{0, c}$ is itself smooth then claims (i) -(iv) hold for $U=\operatorname{Spec} \mathrm{Z}_{0, c}$, and $\mathrm{H}_{U}=\mathrm{H}_{0, c}$.

Following $[\mathrm{Alv}]$, on the group algebra $\mathbb{C} \Gamma$, define an increasing filtration $F_{\bullet}(\mathbb{C} \Gamma)$ by letting $F_{k}(\mathbb{C} \Gamma), k \geq 0$, be the $\mathbb{C}$-linear span of the elements $g \in \Gamma$ such that $\mathrm{rk}(\mathrm{Id}-g) \leq k$. This filtration is obviously compatible with the algebra structure on $\mathbb{C} \Gamma$. Let $F_{\bullet}(Z \Gamma)$ denote the induced filtration on $\mathrm{Z} \Gamma$, the center of $\mathbb{C} \Gamma$. Write $\mathrm{gr}_{\bullet} F(\mathrm{Z} \Gamma)$ for the corresponding associated graded algebra.

Further, write $H H^{\bullet}(\mathcal{A})$ for the Hochschild cohomology of an associative algebra $\mathcal{A}$, and $H^{\bullet}(-)$ for the ordinary singular cohomology of a topological space (with complex coefficients). Part (i) of the theorem below is a generalization of [AFLS] and [Alv], while part (ii), may be viewed as a 'symplectic generalization' of McKay correspondence, cf. $[\operatorname{Re}]$.

Theorem 1.8 For any $c \in \mathrm{C}$ we have:

(i) For all $t \in \mathbb{C}^{*}$, except possibly a countable set, there is a natural graded algebra isomorphism: $H^{\bullet}\left(\mathrm{H}_{t, c}\right) \simeq \mathrm{gr}_{\bullet}^{F}(\mathrm{Z \Gamma})$. 
(ii) If the variety $\operatorname{Spec} \mathrm{Z}_{0, c}$ is smooth, then the Poisson structure on $\mathrm{Z}_{0, c}$ makes Spec $Z_{0, c}$ a symplectic manifold; Furthermore, there is a graded algebra isomorphism: $H^{\bullet}\left(\operatorname{Spec} \mathrm{Z}_{0, c}\right) \simeq \operatorname{gr}_{\bullet} F(\mathrm{Z} \Gamma)$.

Corollary 1.9 If $\operatorname{Spec} Z_{0, c}$ is smooth, then $\operatorname{dim} H^{\text {odd }}\left(\operatorname{Spec} Z_{0, c}\right)=0$, and for all $i \geq 0$, we have: $\operatorname{dim} H^{2 i}\left(\operatorname{Spec} \mathrm{Z}_{0, c}\right)=\mathbf{n}(i)$, where

$$
\mathbf{n}(i):=\text { number of conjugacy classes of } g \in \Gamma \text { such that } \operatorname{rk}(\mathrm{Id}-g)=2 i \text {. }
$$

Recall the result of Batyrev [Ba, Thm. 4.8] saying that, for any crepant (hence, symplectic, see e.g. [Ve]) resolution: $\widehat{V / \Gamma} \rightarrow V / \Gamma$, one has: $\operatorname{dim} H^{2 i}(\widehat{V / \Gamma})=\mathbf{n}(i)$. It has been conjectured by E. Vasserot [Va] (and also by the second author) that, for a resolution $\widehat{V / \Gamma}$ as above, there is a graded algebra isomorphism: $H^{\bullet}(\widehat{V / \Gamma}) \simeq \mathrm{gr}_{\bullet}^{F}(\mathrm{Z} \Gamma)$. This conjecture is related to Theorem 1.8 as follows. According to [Ka2], if the symplectic orbifold $V / \Gamma$ has a smooth symplectic resolution of singularities, $\widehat{V / \Gamma}$, then this resolution can be deformed to a smooth affine symplectic manifold. We expect that the latter coincides with the variety $\operatorname{Spec} Z_{0, c}$, for an appropriate value of the parameter $c \in C$, in particular, Spec $Z_{0, c}$ is smooth for generic $c \in C$. Our expectation is based on Proposition 17.4 (of Appendix F) that would conjecturally imply that any Poisson deformation of $V / \Gamma$, in particular the one constructed by Kaledin, is given by a certain variety $\operatorname{Spec} Z_{0, c}$. If our expectations are correct, then any smooth symplectic resolution, $\widehat{V / \Gamma}$, has the same cohomology algebra as Spec $\mathrm{Z}_{0, c}$. Thus, Theorem 1.8 would imply the conjecture in [Va]. In many concrete examples the space $\widehat{V / \Gamma}$ has a natural hyper-Kähler structure such that Spec $\mathrm{Z}_{0, c}$ is obtained from $\widehat{V / \Gamma}$ by 'rotating' the complex structure. Hence the two spaces are even diffeomorphic, and Theorem 1.8 applies, cf. Question 17.2. This is the case, for instance, for $\Gamma=S_{n}$ acting diagonally on $V=\mathbb{C}^{n} \oplus \mathbb{C}^{n}$. In that case, $\widehat{V / \Gamma}=\operatorname{Hilb}^{n}\left(\mathbb{C}^{2}\right)$ is the Hilbert scheme of $n$ points on the plane, and the corresponding result on the algebra structure of $H^{\bullet}\left(\mathrm{Hilb}^{n}\left(\mathbb{C}^{2}\right)\right)$ has been recently proved in [LSo] and [Va] (independently). Their approach is totally different from ours.

Further, write $K(X)$ for the algebraic $K$-theory of an algebraic variety $X$, that is, for the Grothendieck group formed by algebraic vector bundles on $X$. Now let $X=\operatorname{Spec} Z_{0, c}$, and assume that it is smooth so that the sheaf $\mathcal{R}$ is locally free, by Theorem 1.7(i). We view $\mathcal{R}$ as an algebraic vector bundle on $\operatorname{Spec} Z_{0, c}$. Given an irreducible $\Gamma$-representation $E$, we let $\mathcal{R}^{E}:=E \otimes_{\Gamma} \mathcal{R}=\operatorname{Hom}_{\Gamma}\left(E^{*}, \mathcal{R}\right)$ denote the $E$-isotypic component of $\mathcal{R}$, which is again an algebraic vector bundle on $\operatorname{Spec} Z_{0, c}$.

Proposition 1.10 If the variety Spec $Z_{0, c}$ is smooth, then $K\left(\operatorname{Spec} Z_{0, c}\right)$ is a free abelian group with the basis $\left\{\mathcal{R}^{E}\right\}_{E \in \operatorname{Irreps}(\Gamma)}$. Moreover, the Chern character map gives an isomorphism: $\mathbb{C} \otimes_{\mathbb{Z}} K\left(\operatorname{Spec} \mathrm{Z}_{0, c}\right) \stackrel{\sim}{\longrightarrow} H^{\mathrm{ev}}\left(\operatorname{Spec} \mathrm{Z}_{0, c}\right)$.

Wreath-product case. An infinite series of interesting examples of groups generated by symplectic reflections is provided by a so-called wreath-product construction. Fix a

\footnotetext{
${ }^{1}$ see $\mathrm{Coh}$ for a classification of finite groups generated by symplectic reflections.
} 
finite subgroup $\Gamma \subset S L_{2}(\mathbb{C})$ and an integer $n>1$. We write $L=\mathbb{C}^{2}$ for the tautological 2-dimensional $\Gamma$-module, form the vector space $V=L^{\oplus n}$ with the symplectic structure $\omega_{V}$ induced from the standard one on $L=\mathbb{C}^{2}$, and let $S_{n}$, the Symmetric group, act on $V$ by permutations of the direct summands. The group $\boldsymbol{\Gamma}_{n}:=S_{n} \ltimes(\Gamma \times \Gamma \times \ldots \times \Gamma) \subset S p(V)$ acts naturally on $V=L^{\oplus n}$ and is called the wreath-product of $S_{n}$ with $\Gamma$.

It is known that $\mathrm{C}\left(\boldsymbol{\Gamma}_{n}\right)$, the space of class functions on the set of symplectic reflections in $\boldsymbol{\Gamma}_{n}$, can be identified, cf. (11.1), with $\mathrm{Z} \Gamma$, the center of the group algebra of $\Gamma$. Thus there is a symplectic reflection algebra, $\mathrm{H}_{0, c}\left(\boldsymbol{\Gamma}_{n}\right)$, attached to the triple $\left(V, \omega_{V}, \boldsymbol{\Gamma}_{n}\right)$, and any parameter $c \in \mathrm{Z} \Gamma \simeq \mathrm{C}\left(\boldsymbol{\Gamma}_{n}\right)$.

On the other hand, let $\mathbf{V}=\mathbb{C} \Gamma^{\oplus n}$ be the direct sum of $n$ copies of the regular representation of $\Gamma$. Following Nakajima [Na1,Na2], to any $c \in Z \Gamma$ one associates an affine algebraic variety of quiver data:

$$
\mathbb{M}_{\Gamma}^{c}(\mathbf{V}):=\left\{(\nabla, I, J) \in\left(\operatorname{Hom}_{\mathbb{C}}\left(\mathbf{V}, \mathbf{V} \otimes_{\mathbb{C}} L\right) \oplus \mathbf{V} \oplus \mathbf{V}^{*}\right)^{\Gamma}|[\nabla, \nabla]+I \otimes J=c|_{\mathbf{V}}\right\},
$$

where $I \otimes J \in \operatorname{End}_{\Gamma} \mathbf{V}$, and $[\nabla, \nabla] \in\left(\operatorname{End}_{\Gamma} \mathbf{V}\right) \otimes \bigwedge^{2} L \simeq \operatorname{End}_{\Gamma} \mathbf{V}$. The natural action on $\left(\operatorname{Hom}_{\mathbb{C}}\left(\mathbf{V}, \mathbf{V} \otimes_{\mathbb{C}} L\right) \oplus \mathbf{V} \oplus \mathbf{V}^{*}\right)^{\Gamma}$ of the group $G_{\Gamma}(\mathbf{V})$ of $\Gamma$-equivariant automorphisms of $\mathbf{V}$ preserves the subvariety $\mathbb{M}_{\Gamma}^{c}(\mathbf{V})$. The quotient: $\mathfrak{M}_{\Gamma, n, c}=\mathbb{M}_{\Gamma}^{c}(\mathbf{V}) / G_{\Gamma}(\mathbf{V})$ is a particular case of the Nakajima quiver variety. In general, the quotient is understood in the sense of Geometric Invariant Theory, but in our case the GIT quotient coincides with the naive one because of the following well-known result (which is essentially Proposition 11.11), see also [Na1]

Lemma 1.12 If $c \in \mathrm{Z} \Gamma$ is generic enough, then the induced $G_{\Gamma}(\mathbf{V})$-action on $\mathbb{M}_{\Gamma}^{c}(\mathbf{V})$ is free. Furthermore, $\mathfrak{M}_{\Gamma, n, c}$ is a smooth symplectic affine algebraic variety of dimension $2 n$.

Write $\mathrm{Z}_{0, c}\left(\boldsymbol{\Gamma}_{n}\right)$ for the center of the algebra $\mathrm{H}_{0, c}\left(\boldsymbol{\Gamma}_{n}\right)$. One of our main results is

Theorem 1.13 If $c \in \mathrm{Z} \Gamma$ is generic enough, then $\operatorname{Spec} \mathrm{Z}_{0, c}\left(\boldsymbol{\Gamma}_{n}\right)$ is isomorphic to $\mathfrak{M}_{\Gamma, n, c}$, as a Poisson algebraic variety.

Corollary 1.14 If $c \in \mathrm{Z} \Gamma$ is generic enough, then we have:

(i) $\operatorname{Spec} \mathrm{Z}_{0, c}\left(\boldsymbol{\Gamma}_{n}\right)$ is a smooth symplectic variety.

(ii) Simple $\mathrm{H}_{0, c}\left(\boldsymbol{\Gamma}_{n}\right)$-modules all have dimension $n ! \cdot|\Gamma|^{n}$, and are parametrized by points of the quiver variety $\mathfrak{M}_{\Gamma, n, c}$.

(iii) There is a canonical algebraic vector bundle $\mathcal{R}$ on $\mathfrak{M}_{\Gamma, n, c}$ whose fibers carry the regular representation of the group $\boldsymbol{\Gamma}_{n}$. The classes: $\left\{\mathcal{R}^{E}\right\}_{E \in \operatorname{Irrep}\left(\boldsymbol{\Gamma}_{n}\right)}$ form a basis of $K\left(\mathfrak{M}_{\Gamma, n, c}\right)$.

(iv) There is an algebra isomorphism: $H^{\bullet}\left(\mathfrak{M}_{\Gamma, n, c}\right) \simeq \operatorname{gr}_{\bullet}^{F} \mathbf{Z} \boldsymbol{\Gamma}_{n}$. 
Rational Cherednik algebra. All indecomposable triples $(V, \omega, \Gamma)$ fall into two large groups: (1) those where $V$ is an irreducible $\Gamma$-module, and (2) those where $V=\mathfrak{h} \oplus \mathfrak{h}^{*}$ is a direct sum of two $\Gamma$-stable irreducible Lagrangian subspaces. In the second case, the action on $\mathfrak{h}$ by any symplectic reflection $g \in \Gamma$ has a pointwise fixed codimension 1 hyperplane in $\mathfrak{h}$, hence is a complex reflection in $\mathfrak{h}$. Thus, in case (2), saying that $\Gamma$ is generated by symplectic reflections amounts to saying that $\Gamma=W$ is a finite complex reflection group in a vector space $\mathfrak{h}$, acting on $V=T^{*} \mathfrak{h} \simeq \mathfrak{h}+\mathfrak{h}^{*}$ by induced symplectic automorphisms. Any (complex) reflection $s \in W$ induces the corresponding symplectic reflection of $\mathfrak{h}+\mathfrak{h}^{*}$.

In this paper, we will be mostly interested in the special case where $W$ is the Weyl group of a finite reduced root system $R \subset \mathfrak{h}^{*}$. The set $S$ of reflections in $W$ forms either one or two conjugacy classes, depending on whether all roots $\alpha \in R$ are of the same length or not. Thus, giving a $W$-invariant function $c: S \rightarrow \mathbb{C}$ amounts to giving a map: $\alpha \mapsto c_{\alpha} \in \mathbb{C}$, where $c_{\alpha}$ depends only on the length of $\alpha \in R$.

Let $\mathrm{H}_{t, c}$ be the symplectic reflection algebra associated to a complex number $t \in \mathbb{C}$, a map $c$ as above, and the vector space $V=\mathfrak{h}+\mathfrak{h}^{*}$. Explicitly, write $\alpha^{\vee} \in \mathfrak{h}$ for the coroot corresponding to a root $\alpha \in R$, and $s_{\alpha} \in W$ for the reflection relative to $\alpha$. Then, formula (1.1) shows that the algebra $\mathrm{H}_{t, c}$ is generated by the spaces $\mathfrak{h}, \mathfrak{h}^{*}$, and the group $W$, subject to the following defining relations:

$$
\begin{array}{ll}
w \cdot x \cdot w^{-1}=w(x), w \cdot y \cdot w^{-1}=w(y), & \forall y \in \mathfrak{h}, x \in \mathfrak{h}^{*}, w \in W \\
{\left[x_{1}, x_{2}\right]=0=\left[y_{1}, y_{2}\right],} & \forall y_{1}, y_{2} \in \mathfrak{h}, x_{1}, x_{2} \in \mathfrak{h}^{*} \\
{[y, x]=t \cdot\langle y, x\rangle-\frac{1}{2} \cdot \sum_{\alpha \in R} c_{\alpha} \cdot\langle y, \alpha\rangle\left\langle\alpha^{\vee}, x\right\rangle \cdot s_{\alpha},} & \forall y \in \mathfrak{h}, x \in \mathfrak{h}^{*},
\end{array}
$$

(the factor $\frac{1}{2}$ on the RHS of the last equation accounts to the fact that roots $\alpha$ and $-\alpha$ give rise to the same reflection).

We will refer to the algebra $\mathrm{H}_{t, c}$ above as a rational Cherednik algebra because it is a certain 'rational' degeneration of the double affine Hecke algebra studied by Cherednik [Ch. In the case of type $\mathbf{A}_{\mathbf{1}}$, the algebra $\mathrm{H}_{\kappa}$ was discussed in detail in ChM.

Olshanetsky-Perelomov $[\mathrm{OP}]$, have associated a Calogero-Moser type integrable system to any Weyl group $W$. We show that the space $\operatorname{Spec} Z\left(\mathrm{H}_{0, c}\right)$ for the rational Cherednik algebra attached to $W$ is a natural completion of the phase space of that integrable system. The affine algebraic variety $\operatorname{Spec} \mathrm{Z}\left(\mathrm{H}_{0, c}\right)$ is always an irreducible normal Gorenstein variety with a Poisson structure. We propose to call it the Calogero-Moser space associated to the Weyl group $W$, with parameter $c$. For Weyl groups of type: $\mathbf{A}_{\mathbf{n}}, \mathbf{B}_{\mathbf{n}}, \mathbf{C}_{\mathbf{n}}$ and sufficiently general parameters $c \in \mathrm{C}$, the variety $\operatorname{Spec} \mathrm{Z}\left(\mathrm{H}_{0, c}\right)$ is a smooth symplectic manifold, by Theorem 1.13 .

Relation to the double-affine Hecke algebra. The Cherednik algebra $\mathrm{H}_{t, c}$ may be thought of as the following two-step degeneration of the double affine Hecke algebra $\mathbf{H}$ defined in $[\mathrm{Ch}]$ :

$$
\mathbf{H}=\mathbf{H}^{\text {elliptic }} \rightsquigarrow \mathbf{H}^{\text {trigonometric }} \rightsquigarrow \mathbf{H}^{\text {rational }}=\mathbf{H}_{t, c} .
$$

In more detail, let $G$ be the Lie group corresponding to the Lie algebra $\mathfrak{g}$, and $T$ a maximal torus of $G$. The algebra $\mathbf{H}$ contains the group algebras of two (dual) lattices: 
$X=\operatorname{Hom}\left(\mathbb{C}^{*}, T\right)$ and $Y=\operatorname{Hom}\left(T, \mathbb{C}^{*}\right)$. Degeneration from $\mathbf{H}$ to $\mathbf{H}^{\text {trigonometric }}$ amounts, effectively, to replacing the group algebra of the lattice $X=\operatorname{Hom}\left(\mathbb{C}^{*}, T\right)$ by the Symmetric algebra of the vector space $\operatorname{Hom}_{\mathbb{C}}(\mathbb{C}, \operatorname{Lie} T)=\mathfrak{h}$, while the group algebra of the second lattice $Y$ remains unaffected. Degeneration from $\mathbf{H}^{\text {trigonometric }}$ to $\mathbf{H}^{\text {rational }}=\mathbf{H}_{t, c}$ is obtained similarly, by further replacing the group algebra of the lattice $Y=\operatorname{Hom}\left(T, \mathbb{C}^{*}\right)$ by the Symmetric algebra of the vector space $\operatorname{Hom}_{\mathbb{C}}(\operatorname{Lie} T, \mathbb{C})=\mathfrak{h}^{*}$.

An analogous degeneration pattern applies to other objects considered in this paper, so that many of the constructions of the paper, for $\Gamma$ being a Weyl group, have their 'trigonometric' and 'elliptic' analogues, see (1.21).

Harish-Chandra homomorphism. The goal of Part II is to describe the spherical subalgebra, $\mathbf{e H}_{t, c} \mathbf{e}$, of the symplectic reflection algebra associated to the root system of type A as a 'quantum Hamiltonian reduction' of the algebra $\mathcal{D}\left(\mathfrak{g l}_{n}\right)$ of polynomial differential operators on the Lie algebra $\mathfrak{g l}_{n}$. A similar description conjecturally exists for the spherical subalgebra of the symplectic reflection algebra associated to any wreath-product $\boldsymbol{\Gamma}_{n}$, in which case $\mathfrak{g l}_{n}$ gets replaced by a (vector) space of representations of a Dynkin quiver $\left(\mathfrak{g l}_{n}\right.$ is the space of $n$-dimensional representations of the quiver with one vertex and one edge-loop).

We begin in a greater generality of an arbitrary complex reductive Lie algebra $\mathfrak{g}$. Let $\mathfrak{h}$ be a Cartan subalgebra of $\mathfrak{g}$, and $W$ the Weyl group of $(\mathfrak{g}, \mathfrak{h})$. Let $\mathcal{D}(\mathfrak{g})^{\mathfrak{g}}$ be the algebra of ad $\mathfrak{g}$-invariant polynomial differential operators on $\mathfrak{g}$, and $\mathcal{D}(\mathfrak{h})^{W}$ the algebra of $W$ invariant polynomial differential operators on $\mathfrak{h}$. Write $\Delta_{\mathfrak{g}}$, resp. $\Delta_{\mathfrak{h}}$, for the second order Laplacian on $\mathfrak{g}$, resp. on $\mathfrak{h}$, associated to a nondegenerate invariant bilinear form on $\mathfrak{g}$. In 1964, Harish-Chandra [HC] defined an algebra homomorphism $\Phi: \mathcal{D}(\mathfrak{g})^{\mathfrak{g}} \rightarrow \mathcal{D}(\mathfrak{h})^{W}$, that reduces to the restriction map: $\mathbb{C}[\mathfrak{g}]^{\mathfrak{g}} \rightarrow \mathbb{C}[\mathfrak{h}]^{W},\left.f \mapsto f\right|_{\mathfrak{h}}$, on zero order differential operators, and such that $\Phi\left(\Delta_{\mathfrak{g}}\right)=\Delta_{\mathfrak{h}}$.

In this paper, we construct a 1-parameter deformation of the Harish-Chandra homomorphism $\Phi$ in the special case $\mathfrak{g}=\mathfrak{g l}_{n}$, and show that this deformed homomorphism may (and should) be viewed as an isomorphism between the quantum Hamiltonian reduction of the algebra $\mathcal{D}\left(\mathfrak{g l}_{n}\right)$ and the spherical subalgebra $\mathbf{e H}_{t, c} \mathbf{e}$. To this end, in $\S 6$ we work out the formalism of radial parts of invariant differential operators in a slightly more general framework than treated in classical texts.

Now let $\mathfrak{h}=\mathbb{C}^{n}$ be the Cartan subalgebra of diagonal matrices in $\mathfrak{g}=\mathfrak{g l}_{n}$. Write $x_{1}, \ldots, x_{n}$ for coordinates in $\mathbb{C}^{n}$, and $\mathfrak{h}^{\text {reg }} \subset \mathbb{C}^{n}$ for the open subset of points with pairwise distinct coordinates. In $\S 7$ we apply the formalism of radial parts to construct a family of algebra homomorphisms $\Phi_{k}: \mathcal{D}(\mathfrak{g})^{\mathfrak{g}} \rightarrow \mathcal{D}\left(\mathfrak{h}^{\text {reg }}\right)^{W}$ depending on a parameter $k \in \mathbb{C}$, which specializes to the Harish-Chandra homomorphism $\Phi$ at $k=0$. For each $k \in \mathbb{C}$, this homomorphism $\Phi_{k}$ reduces to the restriction map: $\mathbb{C}[\mathfrak{g}]^{\mathfrak{g}} \rightarrow \mathbb{C}[\mathfrak{h}]^{W},\left.f \mapsto f\right|_{\mathfrak{h}}$, on zero order differential operators. Further, we have: $\Phi_{k}\left(\Delta_{\mathfrak{g}}\right)=\mathrm{L}_{k}$, where

$$
\mathrm{L}_{k}=\sum_{j} \frac{\partial^{2}}{\partial x_{j}^{2}}-\sum_{i \neq j} \frac{k(k+1)}{\left(x_{i}-x_{j}\right)^{2}}
$$

is the Calogero-Moser operator with rational potential, corresponding to the parameter $k$. 
We now describe the image of the map $\Phi_{k}$. Let $\mathcal{D}\left(\mathfrak{h}^{\text {reg }}\right)^{W}$ denote the algebra of $W$ invariant differential operators on $\mathfrak{h}^{\text {reg }}$. Let $\mathcal{D}\left(\mathfrak{h}^{\text {reg }}\right)_{-}^{W}$ denote the algebra spanned by homogeneous elements $D$ (with respect to the $\mathbb{C}^{*}$-action on $\mathfrak{h}^{\text {reg }}$ ) of $\mathcal{D}\left(\mathfrak{h}^{\text {reg }}\right)^{W}$ such that $\operatorname{order}(D)+\operatorname{degree}(D) \leq 0$. Clearly, $\mathrm{L}_{k}$ belongs to $\mathcal{D}\left(\mathfrak{h}^{\text {reg }}\right)_{-}^{W}$. Let $\mathcal{C}_{k}$ denote the centraliser of $\mathrm{L}_{k}$ in the algebra $\mathcal{D}\left(\mathfrak{h}^{\text {reg }}\right)_{-}^{W}$. If $k=0$, then it follows from Lemma 14.6 below that $\mathcal{C}_{0}=S \mathfrak{h}^{W}$, is the algebra of $W$-invariant differential operators with constant coefficients. In general, follows from $\mathrm{O}$ and Lemma 14.6 that for each $k$, there exists an algebra isomorphism $\sigma_{k}: S \mathfrak{h}^{W} \stackrel{\sim}{\sim} \mathcal{C}_{k}$, such that, for any homogeneous element $u \in S \mathfrak{h}^{W}$ of degree $\ell \geq 0$, the differential operator $\sigma_{k}(u)$ has order $\ell$, and its principal symbol equals $u$; moreover, $\sigma_{k}\left(\Delta_{\mathfrak{h}}\right)=\mathrm{L}_{k}$. It turns out that the image of the homomorphism $\Phi_{k}$ equals $\mathcal{B}_{k}$, the associative subalgebra in $\mathcal{D}\left(\mathfrak{h}^{\text {reg }}\right)^{W}$ generated by $\mathcal{C}_{k}$ and by the polynomial subalgebra $\mathbb{C}[\mathfrak{h}]^{W} \subset \mathcal{D}(\mathfrak{h})^{W}$, of zero order $W$-invariant operators on $\mathfrak{h}$.

Thus, we summarize:

Theorem 1.17 There exists a (flat) family of surjective algebra homomorphisms $\Phi_{k}$ : $\mathcal{D}(\mathfrak{g})^{\mathfrak{g}} \rightarrow \mathcal{B}_{k}$, all of which reduce to the restriction map: $\left.f \mapsto f\right|_{\mathfrak{h}}$, on zero order differential operators. This family specializes, at $k=0$, to the Harish-Chandra homomorphism $\Phi$; furthermore, $\Phi_{k}\left(\Delta_{\mathfrak{g}}\right)=\mathrm{L}_{k}$, for any $k \in \mathbb{C}$.

A drawback of Theorem 1.17 is that elements of the algebra $\mathcal{B}_{k} \subset \mathcal{D}\left(\mathfrak{h}^{\text {reg }}\right)^{W}$ may have quite complicated singularites at the divisor $\mathfrak{h} \backslash \mathfrak{h}^{\text {reg }}$. Therefore, it is tricky to give an explicit description of $\mathcal{B}_{k}$, as a subalgebra inside $\mathcal{D}\left(\mathfrak{h}^{\text {reg }}\right)^{W}$. Remarkably, the algebra $\mathcal{B}_{k}$ admits an imbedding into $\mathbf{H}_{\kappa}$, the rational Cherednik algebra of type $\mathbf{A}_{\mathbf{n}-\mathbf{1}}$. The image of $\mathcal{B}_{k}$ has a very simple description that we now explain.

If $R=\mathbf{A}_{\mathbf{n}-1}$, is the root system of $\mathfrak{g}=\mathfrak{g l}_{n}$ then all roots are of the same length, and $\mathbf{C}=$ $\mathbb{C}$. We treat the parameter $\kappa=(t, c)$ as a point on the Riemann sphere $\mathbb{P}^{1}=(\mathbb{C} \oplus \mathbb{C}) / \mathbb{C}^{*}$ with homogeneous coordinates $(c: t)$, i.e., as a complex number $\kappa=c / t \in \mathbb{C} \cup\{\infty\}$ (as will be explained after (2.4), rescaling: $(t, c) \mapsto(r \cdot t, r \cdot c), r \in \mathbb{C}^{*}$, has no essential effect on the algebra $\left.\mathrm{H}_{t, c}\right)$. Thus, in the $\mathbf{A}_{\mathbf{n}-\mathbf{1}}$-case we make no distinction between the parameter: $\kappa=c / t$, and the complex parameter ' $k$ ' entering the deformed Harish-Chandra homomorphism $\Phi_{k}$. Note that the limit $\kappa \rightarrow \infty$ corresponds to $t \rightarrow 0$.

For any Weyl group, Cherednik defined a faithful representation of the double affine Hecke algebra, given by the so-called Demazure-Lusztig-Dunkl operators. A version of Cherednik's construction yields a faithful representation of $H_{\kappa}$ in the vector space $\mathbb{C}\left[\mathfrak{h}^{\text {reg }}\right]$. It is easy to see that the restriction of this representation to the spherical subalgebra $\mathbf{e H}_{\kappa} \mathbf{e} \subset \mathrm{H}_{\kappa}$ keeps the subspace $\mathbb{C}\left[\mathfrak{h}^{\text {reg }}\right]^{W} \subset \mathbb{C}\left[\mathfrak{h}^{\text {reg }}\right]$ stable, and the resulting $\mathbf{e H}_{\kappa} \mathbf{e}$-action on $\mathbb{C}\left[\mathfrak{h}^{\text {reg }}\right]^{W}$ is given by $W$-invariant regular differential operators on $\mathfrak{h}^{\text {reg }}$. This gives an algebra homomorphism

$$
\Theta_{\kappa}^{\text {spher }}: \mathbf{e H}_{\kappa} \mathbf{e} \longrightarrow \mathcal{D}\left(\mathfrak{h}^{\text {reg }}\right)^{W}
$$

Thus, for $\mathfrak{g}=\mathfrak{g l}_{n}$ and $W=S_{n}$, we get the following diagram:

$$
\mathcal{D}(\mathfrak{g})^{\mathfrak{g}} \stackrel{\Phi_{\kappa}}{\longrightarrow} \mathcal{D}\left(\mathfrak{h}^{\mathrm{reg}}\right)^{W} \stackrel{\Theta_{\kappa}^{\text {spher }}}{\longleftarrow} \mathbf{e H}_{\kappa} \mathbf{e}
$$


The remarkable fact is, that the homomorphisms $\Phi_{\kappa}$ and $\Theta_{\kappa}^{\text {spher }}$ above have the same image, the subalgebra $\mathcal{B}_{\kappa} \subset \mathcal{D}\left(\mathfrak{h}^{\text {reg }}\right)^{S_{n}}$. Further, the map $\Theta_{\kappa}^{\text {spher }}$ in $(1.19)$ is a bijection onto its image. Inverting this map, we thus obtain an algebra homomorphism:

$$
\Phi_{\kappa}^{\text {spher }}=\left(\Theta_{\kappa}^{\text {spher }}\right)^{-1} \circ \Phi_{\kappa}: \quad \mathcal{D}\left(\mathfrak{g l}_{n}\right)^{\mathfrak{g l}_{n}} \longrightarrow \mathbf{e H}_{\kappa} \mathbf{e}
$$

This homomorphism provides a link between invariant differential operators on $\mathfrak{g l}_{n}$ and the Cherednik algebra $\mathrm{H}_{\kappa}$; it plays a crucial role in this paper. Furthermore, replacing the Lie algebra $\mathfrak{g}=\mathfrak{g l}_{n}$ by the group $G=G L_{n}$, one can define a 'trigonometric' version of the deformed Harish-Chandra homomorphism (1.20):

$$
\Phi_{\kappa}^{\text {trigonometric }}: \mathcal{D}(G)^{G} \longrightarrow \mathbf{e} \cdot \mathbf{H}^{\text {trigonometric }} \cdot \mathbf{e} .
$$

One might expect that replacing further $\mathcal{D}(G)$ by the so-called Heisenberg double of the corresponding quantum group, see e.g. STS, one gets an elliptic analogue of the sperical Harish-Chandra homomorphism with image eHe. This agrees well with the results of [EK], although we do not pursue this line here.

Calogero-Moser space. It is a well-known principle that the 'classical' limit of the algebra $\mathcal{D}(\mathfrak{g})$ is the commutative algebra of regular functions on $T^{*} \mathfrak{g}$, the cotangent bundle on $\mathfrak{g}$. Since $T^{*} \mathfrak{g}=\mathfrak{g} \oplus \mathfrak{g}^{*} \simeq \mathfrak{g} \oplus \mathfrak{g}$, the 'classical' limit of $\mathcal{D}(\mathfrak{g})^{\mathfrak{g}}$ is the algebra $\mathbb{C}[\mathfrak{g} \oplus \mathfrak{g}]^{\mathfrak{g}}$ of ad $\mathfrak{g}$-invariant polynomials on $\mathfrak{g} \oplus \mathfrak{g}$. Now, let $\mathfrak{g}=\mathfrak{g l}_{n}$. There are reasons to think of the limit: $\kappa=(t, c) \longrightarrow \infty=(0,1) \in \mathbb{P}^{1}$, as a 'classical' limit. Accordingly, we show that the family of Harish-Chandra homomorphisms $\Phi_{\kappa}^{\text {spher }}: \mathcal{D}(\mathfrak{g})^{\mathfrak{g}} \rightarrow \mathbf{e H}_{\kappa} \mathbf{e}$, see (1.20), has a 'classical' limit, a surjective algebra homomorphism $\Phi_{\infty}^{\text {spher }}: \mathbb{C}[\mathfrak{g} \oplus \mathfrak{g}]^{\mathfrak{g}} \rightarrow \mathbf{e H}_{\infty} \mathbf{e}$. It turns out that this homomorphism is intimately related to the so-called Calogero-Moser space $\mathcal{M}_{n}$, a symplectic algebraic manifold introduced by Kazhdan-Kostant-Sternberg [KKS], and studied further by G. Wilson [Wi]. This space, which is a particular case of the quiver variety $\mathfrak{M}_{\Gamma, n, c}$, for $\Gamma=\{1\}$, is defined as a categorical quotient: $\mathcal{M}_{n}=M / / \mathrm{Ad}_{\mathrm{GL}}$, where

$$
M:=\{(X, Y) \in \mathfrak{g} \times \mathfrak{g} \mid[X, Y]+\mathrm{Id}=\operatorname{rank} 1 \text { matrix }\},
$$

is an affine subvariety in $\mathfrak{g} \oplus \mathfrak{g}$, a special case of the quiver data variety $\mathbb{M}_{\Gamma}^{c}(\mathbf{V})$. Thus, by definition, the coordinate ring of $\mathcal{M}_{n}$ equals $\mathbb{C}\left[\mathcal{M}_{n}\right]=\mathbb{C}[\mathfrak{g} \oplus \mathfrak{g}]^{\mathfrak{g}} /\left(\mathcal{I}_{M}\right)^{\mathfrak{g}}$, where $\mathcal{I}_{M} \subset$ $\mathbb{C}[\mathfrak{g} \oplus \mathfrak{g}]$ denotes the defining ideal of $M$, and the superscript ' $\mathfrak{g}$ ' stands for 'ad $\mathfrak{g}$-invariants'. A 'commutative' counterpart of homomorphism (1.20) reads:

Theorem 1.23 The kernel of the homomorphism $\Phi_{\infty}^{\text {spher }}$ equals $\left(\mathcal{I}_{M}\right)^{\mathfrak{g}}$; hence one has the following chain of Poisson algebra isomorphisms:

$$
\mathbb{C}\left[\mathcal{M}_{n}\right]=\mathbb{C}[\mathfrak{g} \oplus \mathfrak{g}]^{\mathfrak{g}} / \operatorname{Ker}\left(\Phi_{\infty}^{\text {spher }}\right) \stackrel{\substack{\Phi^{\text {spher }} \\ \sim}}{\longrightarrow} \mathbf{e H}_{\infty} \mathbf{e} \stackrel{\text { Satake }-1}{\sim} \underset{Z}{\longrightarrow}\left(\mathrm{H}_{\infty}\right)
$$

This result provides, in the special case $\Gamma=\{1\}$, a transparent conceptual interpretation of the isomorphism of Theorem 1.13. Thus, for $\boldsymbol{\Gamma}_{n}=S_{n}$ and $\kappa=\infty$, Corollary 1.14 yields 
Theorem 1.24 If $W=S_{n}$ is the Symmetric group and $\kappa=\infty=(0, c), c \neq 0$, then

(i) The algebra $\mathrm{H}_{\infty}=\mathrm{H}_{0, c}\left(S_{n}\right)$ is Morita equivalent to the algebra: $\mathrm{Z}_{\infty} \simeq \mathbb{C}\left[\mathcal{M}_{n}\right]$.

(ii) Any simple $\mathrm{H}_{\infty}$-module is isomorphic to one of the form: $\mathrm{H}_{\infty} \mathbf{e} \otimes_{\mathbf{z}_{\infty}} \chi$, for a certain character $\chi: \mathbf{Z}_{\infty} \rightarrow \mathbb{C}$. Thus, the isomorphism classes of simple $\mathbf{H}_{\infty}$-modules are parametrized by points of the Calogero-Moser space $\mathcal{M}_{n}$.

(iii) Any simple $\mathrm{H}_{\infty}$-module has dimension $n$ ! and is isomorphic, as an $S_{n}$-module, to the regular representation of $S_{n}$.

Berest and Wilson have introduced an (infinite-dimensional) group $\mathbb{G}$ of the automorphisms of $\mathbb{C}\langle x, y\rangle$, the free associative algebra on two generators, that keep the element $x y-y x \in \mathbb{C}\langle x, y\rangle$ fixed. We show that the group $\mathbb{G}$ acts naturally on $\mathcal{D}\left(\mathfrak{g l}_{n}\right)$ and on $\mathrm{H}_{\kappa}$ by algebra automorphisms, preserving subalgebras $\mathcal{D}\left(\mathfrak{g l}_{n}\right)^{\mathfrak{g l}_{n}}$ and $\mathbf{e H}_{\kappa} \mathbf{e}$, respectively. Moreover, the map $\Phi_{\kappa}^{\text {spher }}: \mathcal{D}\left(\mathfrak{g l}_{n}\right)^{\mathfrak{g l}_{n}} \longrightarrow \mathbf{e H}_{\kappa} \mathbf{e}$ commutes with the $\mathbb{G}$-action. Further, in $[\mathrm{BW}]$, Berest-Wilson defined a transitive action of $\mathbb{G}$ on the Calogero-Moser space $\mathcal{M}_{n}$. It follows from our results that the $\mathbb{G}$-action on $\mathcal{M}_{n}$ can be canonically lifted to a $\mathbb{G}$-action on the vector bundle $\mathcal{R}$, see Theorem 1.7. It seems to us quite important to obtain a better geometric understanding of this vector bundle.

Guide for the reader. Theorem 1.3, Theorem 1.6, and part (i) of Theorem 1.8 are proved in $\S 2$. Theorem 1.5, Theorem 1.7, part (ii) of Theorem 1.8, and Proposition 1.10 are proved in $\S 3$. For the proof of the Satake isomorphism see Theorem 3.1, and normality of Spec $Z_{0, c}$ is Lemma 3.5. Rational Cherednik algebras are discussed in $\S 4$. Theorem 1.13 about the structure of $\operatorname{Spec} Z_{0, c}$ for wreath-products is a combination of Theorem 11.16, and Theorem 16.1 (Appendix E). The image and the kernel of the deformed HarishChandra homomorphism $\Phi_{k}$ are described in Theorem 7.3, which contains in particular Theorem 1.17 as part of the statement. The proof of Theorem 7.3 is given in $\S 10$, but it depends in an essential way on Theorem 9.1, and on Theorem 6.8, proved in $\S 9$ and in references [LS1],[LS2],[Wa], respectively. Finally, Theorem 1.23 is Theorem 11.29(ii).

Acknowledgments. We are very grateful to A. Braverman for many discussions and helping us with a number of proofs. We thank I. Cherednik, V. Drinfeld, D. Gaitsgory, and V. Vologodsky for useful discussions. We are especially indebted to Bert Kostant for communicating to us his (partly unpublished) results, which we reproduced in Appendix G. The second author thanks T. Stafford for consultations on non-commutative algebra. The work of the first author was partly conducted for the Clay Mathematics Institute, and was partially supported by the NSF grants DMS-9700477 and DMS-9988796.

\section{PART 1. SYMPLECTIC REFLECTION ALGEBRAS}

\section{Koszul patterns and deformations}

Recall the setup outlined in the Introduction. Thus $(V, \omega)$ is a finite dimensional symplectic vector space, and $\Gamma \subset S p(V)$ is a finite group, such that the triple $(V, \omega, \Gamma)$ is indecomposable, i.e., there is no $\omega$-orthogonal direct sum decomposition: $V=V_{1} \oplus V_{2}$, 
where $V_{i}$ are $\Gamma$-stable proper symplectic vector subspaces in $V$. Schur lemma implies that there are exactly two situations in which $(V, \omega, \Gamma)$ is indecomposable:

- $\quad V$ is an irreducible $\Gamma$-module of quaternionic type, i.e. $\left(\Lambda^{2} V^{*}\right)^{\Gamma} \neq 0$.

- $V=\mathfrak{h} \oplus \mathfrak{h}^{*}$, where $\mathfrak{h}$ is an irreducible $\Gamma$-module of either real or complex type.

An application of Schur lemma shows that a triple $(V, \omega, \Gamma)$ is indecomposable if and only if the space $\left(\Lambda^{2} V^{*}\right)^{\Gamma}$ is 1-dimensional, in which case this space is spanned by $\omega$.

Given a skew-symmetric $\mathbb{C}$-bilinear pairing $\kappa: V \times V \rightarrow \mathbb{C} \Gamma$, we introduce an associative algebra $\mathrm{H}_{\kappa}$ as defined in (11.1).

Proof of Theorem 1.3: First of all, it is clear that a necessary condition for the PBWproperty is that the form $\kappa$ is $\Gamma$-invariant (where $\Gamma$ acts on itself by conjugation); otherwise the PBW property fails already in degree two of the filtration. Thus from now on we will assume that $\kappa$ is $\Gamma$-invariant.

Write: $v \mapsto v^{g}$ for the action of $g \in \Gamma$ on $V$. Set $K=\mathbb{C} \Gamma$, and let $E=V \otimes_{\mathbb{C}} \mathbb{C} \Gamma$. The space $E$ has a natural $K$-bimodule structure, with left $\Gamma$-action given by $g: v \otimes a \mapsto$ $v^{g} \otimes(g a)$, and right $\Gamma$-action given by $g: v \otimes a \mapsto v \otimes(a g)$. Let $T_{K} E=\bigoplus_{i} T_{K}^{i} E=K \oplus E \oplus$ $\left(E \otimes_{K} E\right) \oplus \ldots$ denote the tensor algebra of the $K$-bimodule $E$. We observe that, for any $i \geq 0$, there is a natural isomorphism: $T_{K}^{i} E \simeq\left(T_{\mathbb{C}}^{i} V\right) \otimes_{\mathbb{C}} \mathbb{C} \Gamma$. This gives a canonical graded algebra isomorphism: $T_{K} E \simeq(T V) \# \Gamma$. Thus we can write: $\mathrm{H}_{\kappa}=T_{K} E / I\langle P\rangle$, where $I\langle P\rangle$ is the two-sided ideal in $T_{K} E$ generated by a certain $K$-bimodule $P \subset K \oplus\left(E \otimes_{K} E\right)$. Explicitly, write $E \wedge E$ for the $K$-subbimodule in $E \otimes_{K} E=V \otimes_{\mathbb{C}} V \otimes_{\mathbb{C}} \mathbb{C} \Gamma$ spanned by elements of the form: $x \otimes y \otimes g-y \otimes x \otimes g, x, y \in V, g \in \Gamma$, and let $\kappa_{K}$ be the (unique) $K$-bimodule map: $E \wedge E \rightarrow K$ that extends the pairing $\kappa: V \otimes V \rightarrow K$. Then we have:

$$
P=\left\{-\kappa_{K}(p)+p \in K \oplus\left(E \otimes_{K} E\right) \quad \mid \quad p \in E \wedge E\right\} .
$$

We see that $\mathrm{H}_{\kappa}=T_{K} E / I\langle P\rangle$ is a nonhomogeneous quadratic $K$-algebra, in the terminology of $[\mathrm{BG}]$ (Braverman and Gaitsgory only consider algebras over a field but, as explained e.g. in [BGS, n.2.7], everything works for quadratic algebras over any ground ring $R$, provided $R$ is a finite dimensional semisimple $\mathbb{C}$-algebra, e.g., $R=\mathbb{C} \Gamma$ ). Similarly, we have: $S V \# \Gamma \simeq T_{K} E / I\langle E \wedge E\rangle$, is a homogeneous quadratic $K$-algebra. Moreover, since $S V$ is a Koszul $\mathbb{C}$-algebra, cf. [BGS], it is easy to deduce that $S V \# \Gamma$ is a Koszul $K$-algebra. Thus, to prove that the PBW-property holds for $\mathrm{H}_{\kappa}=T_{K} E / I\langle P\rangle$ we may apply (a ' $K$-version' of) the criterion of $[\mathrm{BG}]$; similar criteria have been obtained earlier by Drinfeld [Dr1], and Polishchuk-Positselsky [PP]. According to [BG, Thm. 4.1] we must verify conditions (i)-(iii) of [BG, Lemma 3.3]. In our situation, conditions (i) and (iii) of that lemma become vacuous, and condition (ii) reads:

$$
\kappa_{K} \otimes_{K} \operatorname{Id}_{E}-\operatorname{Id}_{E} \otimes_{K} \kappa_{K} \text { vanishes on }(E \wedge E) \otimes_{K} E \bigcap E \otimes_{K}(E \wedge E) \subset T_{K}^{3} E \text {. }
$$

Now, it is straightforward to see that $(E \wedge E) \otimes_{K} E \cap E \otimes_{K}(E \wedge E)=\Lambda^{3} V \otimes_{\mathbb{C}} \mathbb{C} \Gamma$, where $\Lambda^{3} V \subset T^{3} V$ denotes the subspace of totally skew-symmetric tensors. Furthermore, it is clear that condition (2.1) amounts to the identity $\operatorname{Alt}([x y] z-x[y z])=0$, where $[a, b]$ 
stands for $a \cdot b-b \cdot a$. The latter identity is the standard Jacobi identity: $[z,[x, y]]=$ $[[z, x], y]+[x,[z, y]]$. Thus, we find that 2.1 is equivalent to that

$$
[z, \kappa(x, y)]=[\kappa(z, x), y]+[x, \kappa(z, y)] \quad, \quad \forall x, y, z \in V .
$$

To study (2.2) in more detail, write $\kappa(x, y)=\sum_{g \in \Gamma} b(g, x, y) \cdot g$, for some $b(g, x, y) \in \mathbb{C}$. We claim $b(g, x, y)=0$ unless $g=1$ or $g \in S$. Using an identity: $[z, g]=z \cdot g-g \cdot z$ $=z \cdot g-g \cdot z \cdot g^{-1} \cdot g=\left(z-z^{g}\right) \cdot g$, we find:

$$
[z,[x, y]]=\sum_{g \in \Gamma} b(g, x, y) \cdot[z, g]=\sum_{g \in \Gamma} b(g, x, y) \cdot\left(z-z^{g}\right) \cdot g
$$

Writing similar expressions for $[[z, x], y]$ and $[x,[z, y]]$, from $(2.2)$ we deduce

$$
\sum_{g} b(g, x, y) \cdot\left(z-z^{g}\right) \cdot g=\sum_{g} b(g, z, x) \cdot\left(y^{g}-y\right) \cdot g+\sum_{g} b(g, z, y) \cdot\left(x-x^{g}\right) \cdot g .
$$

Therefore, for each $g \in \Gamma$ we must have:

$$
b(g, x, y) \cdot\left(z-z^{g}\right)=b(g, z, x) \cdot\left(y^{g}-y\right)+b(g, z, y) \cdot\left(x-x^{g}\right) \quad, \quad x, y, z \in V
$$

Fix $g \neq 1$ and assume $b(g, x, y)$ is not identically zero. Choosing $x, y$ generic enough so that $b(g, x, y) \neq 0$, we see that $\left(y-y^{g}\right)$ and $\left(x-x^{g}\right)$ span $\operatorname{Im}(\operatorname{Id}-g)$. Hence $g \in S$.

Fix $s \in S$. The assignment: $x, y \mapsto b(s, x, y)$ gives a skew-symmetric bilinear form: $V \times V \rightarrow \mathbb{C}$. Assuming this form is non-zero, we find $x, y \in V$ such that $b(s, x, y) \neq 0$. Then, for $g=s$ and $z \in \operatorname{Ker}(\operatorname{Id}-s)$, equation (2.3) yields: $b(s, x, z)=0=b(s, y, z)$. This proves that the space $\operatorname{Ker}(\operatorname{Id}-s)$ is contained in the radical of the bilinear form: $x, y \mapsto b(s, x, y)$. Hence, this form is proportional to $\omega_{s}$, since any two 2-forms on the two-dimensional vector space $\operatorname{Im}(\operatorname{Id}-s)$ must be proportional.

Thus, $\Gamma$-equivariance of the pairing $\kappa$ implies the existence of an Ad $\Gamma$-invariant function $c: S \rightarrow \mathbb{C}$ and an Ad $\Gamma$-invariant skew-symmetric bilinear form $f: V \times V \rightarrow \mathbb{C}$ such that we have

$$
\kappa(x, y)=f(x, y) \cdot 1+\sum_{s \in S} c_{s} \cdot \omega_{s}(x, y) \cdot s \quad, \quad \forall x, y \in V .
$$

The form $f$ belongs to $\left(\Lambda^{2} V^{*}\right)^{\Gamma}$ and therefore is proportional to $\omega$, since this space is one dimensional, by indecomposability of the triple $(V, \omega, \Gamma)$.

Let $\mathrm{C}$ denote the vector space of $\mathrm{Ad} \Gamma$-invariant functions on $S$, so that:

$$
\operatorname{dim} \mathrm{C}=\text { number of Ad } \Gamma \text {-conjugacy classes in } S \text {. }
$$

From now on, we assume that the pairing $\kappa$ in (1.1) has the form described in Theorem 1.3, for a certain $t \in \mathbb{C}$ and $c \in \mathrm{C}$. The corresponding algebra $\mathrm{H}_{t, c}:=\mathrm{H}_{\kappa}$ will be referred to as a symplectic reflection algebra. Thus, Theorem 1.3 says:

$$
\operatorname{gr}\left(\mathrm{H}_{t, c}\right) \simeq S V \# \Gamma, \text { and } \mathrm{H}_{0,0}=S V \# \Gamma .
$$

We note that, for any $r \in \mathbb{C}^{*}$, the rescaling map: $V \rightarrow V, v \mapsto \sqrt{r} \cdot v$, gives an algebra isomorphism: $\mathrm{H}_{r \cdot t, r \cdot c} \stackrel{\sim}{\longrightarrow} \mathrm{H}_{t, c}$. Thus, the family of algebras $\left\{\mathrm{H}_{t, c},(t, c) \in(\mathbb{C} \oplus\right.$ 
C) $\backslash\{(0,0)\}\}$ is parametrized, effectively, by the projective space $\bar{C}:=(\mathbb{C} \oplus \mathrm{C}) / \mathbb{C}^{*}$ of $\operatorname{dimension} \operatorname{dim} C$. Points of the form $(0, c) \in \overline{\mathrm{C}}$ will play an especially important role.

Remark. Define an oriented quiver $\mathbb{Q}$ whose vertex set $I$ is the set of (isomorphism classes of) simple $\Gamma$-modules. Given two vertices, i.e. two simple $\Gamma$-modules $\rho, \sigma \in I$, we let $\operatorname{dim} \operatorname{Hom}_{\Gamma}(\rho \otimes V, \sigma)$ be the number of arrows in $\mathbb{Q}$ with head $\rho$ and tail $\sigma$. Note that since $V$ is a self-dual $\Gamma$-module, the number of arrows from $\rho$ to $\sigma$ equals the number of arrows from $\sigma$ to $\rho$. One can show, mimicking the argument in [CBH, $\S 3]$, that the algebra $\mathrm{H}_{t, c}$ is Morita equivalent to a proper quotient of a suitable deformed preprojective algebra associated to $\mathbb{Q}$ (viewed as the double of some other quiver), see $[\mathrm{CBH}]$. For a general group $\Gamma \subset S p(V)$, the quiver $\mathbb{Q}$ is quite complicated, as illustrated by the following example.

Let $\Gamma=S_{n}$ be the Symmetric group acting on $V=\mathbb{C}^{n-1} \oplus \mathbb{C}^{n-1}$, a sum of two copies of the permutation representation in $\mathbb{C}^{n-1}=\left\{\left(x_{1}, \ldots, x_{n}\right) \in \mathbb{C}^{n} \mid \sum x_{i}=0\right\}$. Then one has:

$$
\operatorname{dim} \operatorname{Hom}_{\Gamma}\left(\rho \otimes \mathbb{C}^{n-1}, \sigma\right)= \begin{cases}N-1 & \text { if } \rho=\sigma \\ 1 & \text { if } \rho \text { and } \sigma \text { are adjacent } \\ 0 & \text { otherwise }\end{cases}
$$

where $N$ is the number of (outer) corners in the Young diagram corresponding to $\rho$, and two representations are said to be adjacent if the corresponding Young diagrams are obtained from each other by moving one corner box from one place to another.

Proof of part (i) of Theorem 1.8. We follow the notation of the proof of Theorem 1.3, in particular we have: $\mathrm{H}_{\kappa}=T_{K} E / I\langle P\rangle$, a nonhomogeneous Koszul $K$-algebra. Let $\Lambda^{p} V \subset$ $V^{\otimes p}$ denote the $\Gamma$-submodule of totally skew-symmetric $p$-tensors, and write $\Lambda^{p} E:=$ $\Lambda^{p} V \otimes_{\mathbb{C}} \mathbb{C} \Gamma \subset T_{K}^{p} E$ for the corresponding $\Gamma$-bimodule.

To compute Hochschild cohomology we will use a Koszul type complex of the form

$$
\ldots \stackrel{\partial}{\longrightarrow} \mathrm{H}_{\kappa} \otimes_{\mathbb{C} \Gamma} \Lambda^{2} E \otimes_{\mathbb{C} \Gamma} \mathrm{H}_{\kappa} \stackrel{\partial}{\longrightarrow} \mathrm{H}_{\kappa} \otimes_{\mathbb{C} \Gamma} E \otimes_{\mathbb{C} \Gamma} \mathrm{H}_{\kappa} \stackrel{\partial}{\longrightarrow} \mathrm{H}_{\kappa} \otimes_{\mathbb{C} \Gamma} \mathrm{H}_{\kappa} \stackrel{\epsilon}{\rightarrow} \mathrm{H}_{\kappa}
$$

where the augmentation $\epsilon$ is induced by multiplication in the algebra $\mathrm{H}_{\kappa}$, and the differential $\partial$ is defined by the formula

$$
\begin{gathered}
\partial: h \otimes v_{1} \wedge \ldots \wedge v_{p} \otimes h^{\prime} \mapsto \sum_{j=1}^{p}(-1)^{j-1}\left(h v_{j} \otimes v_{1} \wedge \ldots \wedge \widehat{v}_{j} \wedge \ldots \wedge v_{p} \otimes h^{\prime}\right. \\
\left.-h \otimes v_{1} \wedge \ldots \wedge \widehat{v}_{j} \wedge \ldots \wedge v_{p} \otimes v_{j} h^{\prime}\right) \quad, \quad h, h^{\prime} \in \mathrm{H}_{\kappa}, v_{1}, \ldots v_{p} \in V .
\end{gathered}
$$

We claim that although, in general, $v_{i} \cdot v_{j} \neq v_{j} \cdot v_{i}$ in $\mathrm{H}_{\kappa}$, we have $\partial \circ \partial=0$, that is (2.5) is indeed a complex. To verify the claim, for any $p \geq 1$ and a subset $D \subset\{1,2, \ldots, p+1\}$, put: $\boldsymbol{\Delta}_{D}=\bigwedge_{\ell \in\{1,2, \ldots, p+1\} \backslash D} v_{\ell} \in \Lambda^{\bullet} V$; in particular, for $D=\{i, j\}$, where $1 \leq i<j \leq p+1$, we have:

$$
\boldsymbol{\Delta}_{\{i, j\}}=v_{1} \wedge \ldots \wedge \widehat{v}_{i} \wedge \ldots \wedge \widehat{v}_{j} \wedge \ldots \wedge v_{p+1} .
$$


We compute

$$
\begin{aligned}
\partial \circ \partial\left(h \otimes v_{1} \wedge\right. & \left.\ldots \wedge v_{p+1} \otimes h^{\prime}\right)=\sum_{1 \leq i<j \leq p+1}(-1)^{i+j-1}\left(h v_{i} v_{j} \otimes \boldsymbol{\Delta}_{\{i, j\}} \otimes h^{\prime}-\right. \\
& \left.-h \otimes \boldsymbol{\Delta}_{\{i, j\}} \otimes v_{i} v_{j} h^{\prime}-h v_{j} v_{i} \otimes \boldsymbol{\Delta}_{\{i, j\}} \otimes h^{\prime}-h \otimes \boldsymbol{\Delta}_{\{i, j\}} \otimes v_{j} v_{i} h^{\prime}\right) \\
= & \sum_{1 \leq i<j \leq p+1}(-1)^{i+j-1}\left(\left(h \cdot \kappa\left(v_{i}, v_{j}\right)\right) \otimes \boldsymbol{\Delta}_{\{i, j\}} \otimes h^{\prime}-h \otimes \boldsymbol{\Delta}_{\{i, j\}} \otimes\left(\kappa\left(v_{i}, v_{j}\right) \cdot h^{\prime}\right)\right) .
\end{aligned}
$$

Now, a Leibniz type formula says that, for each $n \geq 0$ and any $u_{1}, \ldots, u_{n} \in V$ and $a \in \mathbb{C} \Gamma$, in $\mathrm{H}_{\kappa} \otimes_{\mathrm{C \Gamma}} \Lambda^{n} E \otimes_{\mathrm{C \Gamma}} \mathrm{H}_{\kappa}$ one has:

$a \otimes u_{1} \wedge \ldots \wedge u_{n} \otimes 1-1 \otimes u_{1} \wedge \ldots \wedge u_{n} \otimes a=\sum_{j=1}^{n} u_{1} \wedge \ldots u_{j-1} \wedge\left[a, u_{j}\right] \wedge u_{j+1} \wedge \ldots u_{n}$

where $[a, u]$ stands for $a \cdot u-u \cdot a$. Hence, the last sum in (2.7) reads

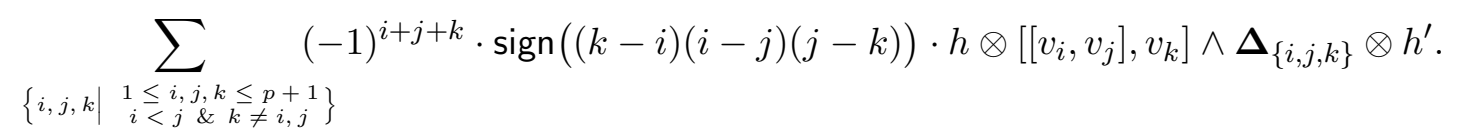

This sum vanishes due to Jacobi identity (2.2). Thus, (2.5) is a complex.

We remark that each term in (2.5) is a projective (but not free) $\mathrm{H}_{\kappa}$-bimodule, and that the equation $\partial \circ \partial=0$ would fail if we had taken in (2.5) tensor products over $\mathbb{C}$ instead of tensor products over $\mathbb{C} \Gamma$. Further, it is straightforward to check that the complex obtained from (2.5) by passing to the associated graded algebras, $\mathrm{grH}_{\kappa}$, is the standard Koszul complex, hence, is exact. It follows that (2.5) is a projective resolution of $\mathrm{H}_{\kappa}$, viewed as an $\mathrm{H}_{\kappa}$-bimodule. Thus, the groups $\operatorname{Ext}_{\mathrm{H}_{\kappa} \text {-bimod }}^{\bullet}\left(\mathrm{H}_{\kappa}, \mathrm{H}_{\kappa}\right)$, may be computed as cohomology groups of the complex with the terms

$$
\operatorname{Hom}_{\mathrm{H}_{\kappa}-\text { bimod }}\left(\mathrm{H}_{\kappa} \otimes_{\mathbb{C} \Gamma} \Lambda^{p} E \otimes_{\mathbb{C} \Gamma} \mathrm{H}_{\kappa}, \mathrm{H}_{\kappa}\right)=\operatorname{Hom}_{\Gamma}\left(\Lambda^{p} V, \mathrm{H}_{\kappa}\right),
$$

where elements $g \in \Gamma$ act on $\mathrm{H}_{\kappa}$ by conjugation: $h \mapsto g \cdot h \cdot g^{-1}$. We conclude that Hochschild cohomology of $\mathrm{H}_{\kappa}$ may be computed using the complex:

$$
\ldots \stackrel{\partial^{\star}}{\longrightarrow} \operatorname{Hom}_{\Gamma}\left(\Lambda^{p} V, \mathrm{H}_{\kappa}\right) \stackrel{\partial^{\star}}{\longrightarrow} \operatorname{Hom}_{\Gamma}\left(\Lambda^{p+1} V, \mathrm{H}_{\kappa}\right) \stackrel{\partial^{\star}}{\longrightarrow} \ldots
$$

with the following differential, $\partial^{\star}$, induced by (2.6):

$\partial^{\star} \beta: v_{1} \wedge \ldots \wedge v_{p+1} \mapsto \sum_{j=1}^{p}(-1)^{j-1}\left[v_{j}, \beta\left(v_{1}, \ldots, \widehat{v}_{j}, \ldots, v_{p}\right)\right], \beta \in \operatorname{Hom}_{\Gamma}\left(\Lambda^{p} V, \mathrm{H}_{\kappa}\right)$

The algebra structure on $\mathrm{H}_{\kappa}$ makes the graded vector space:

$$
\operatorname{Hom}_{\Gamma}\left(\Lambda^{\bullet} V, \mathrm{H}_{\kappa}\right):=\bigoplus_{n \geq 0} \operatorname{Hom}_{\Gamma}\left(\Lambda^{n} V, \mathrm{H}_{\kappa}\right) \simeq\left(\oplus_{n \geq 0} \Lambda^{n} V^{*}\right) \bigotimes_{\mathbb{C \Gamma}} \mathrm{H}_{\kappa}
$$

into a differential graded algebra. Moreover, one veriefies in a standard way that the induced cup-product on the cohomology of this algebra coincides with the Yoneda (equivalently, cup-) product on the Ext-algebra Ext ${ }_{\mathrm{H}_{\kappa}-\text { bimod }}^{\bullet}\left(\mathrm{H}_{\kappa}, \mathrm{H}_{\kappa}\right)$. 
Now given $g \in \Gamma$, set: $V_{\text {Ker }}=\operatorname{Ker}(\operatorname{Id}-g) \subset V$, resp. $V_{\mathrm{Im}}=\operatorname{Im}(\operatorname{Id}-g)$. Thus, $V=V_{\mathrm{Im}} \oplus V_{\mathrm{ker}}$, and if $\operatorname{dim} V_{\mathrm{Im}}=2 d(g)$, then we have the corresponding decomposition: $\Lambda^{2 d(g)} V=\bigoplus_{p+q=2 d(g)} \Lambda^{p} V_{\text {Im }} \otimes \Lambda^{q} V_{\text {Ker }}$. We let $\omega_{g}$ be the $2 d(g)$-form on $V$ whose restriction to the line $\Lambda^{2 d(g)} V_{\mathrm{Im}}$ coincides with $\left.\wedge^{d(g)} \omega\right|_{\Lambda^{2 d(g)} V_{\mathrm{Im}}}$, and that vanishes on any other direct summand $\Lambda^{p} V_{\mathrm{Im}} \otimes \Lambda^{q} V_{\mathrm{Ker}}, q>0$, in the decomposition above. Thus, for $g \in S$ a symplectic reflection, $\omega_{g}$ is the 2-form involved in the bilinear form $\kappa$, see Theorem 1.3. Further, following an idea of Alvarez [Alv], for each $g \in \Gamma$, we put $\psi_{g}:=\omega_{g} \otimes g \in \Lambda^{2 d(g)} V^{*} \otimes \mathrm{H}_{\kappa}=$ $\operatorname{Hom}_{\mathbb{C}}\left(\Lambda^{2 d(g)} V, \mathrm{H}_{\kappa}\right)$.

We remark that although the element $\psi_{g}, g \in \Gamma$, is not a $\Gamma$-equivariant map, i.e. not an element of $\left(\operatorname{Hom}_{\mathbb{C}}\left(\Lambda^{\bullet} V, \mathrm{H}_{\kappa}\right)\right)^{\Gamma}=\operatorname{Hom}_{\Gamma}\left(\Lambda^{\bullet} V, \mathrm{H}_{\kappa}\right)$, one can still formally apply the differential $\partial^{\star}$ given by $(2.9)$ to $\psi_{g}$ (the reader should be warned that the map $\partial^{\star}$ : $\operatorname{Hom}_{\mathbb{C}}\left(\Lambda^{\bullet} V, \mathrm{H}_{\kappa}\right) \rightarrow \operatorname{Hom}_{\mathbb{C}}\left(\Lambda^{\bullet+1} V, \mathrm{H}_{\kappa}\right)$ does not however square to zero). We claim that $\psi_{g}$ is a 'cocycle', that is:

$$
\partial^{\star} \psi_{g}=0 \quad, \quad \forall g \in \Gamma .
$$

To see this, it suffices to check the equation: $\partial^{\star} \psi_{g}\left(v_{1} \wedge \ldots \wedge v_{2 d(g)+1}\right)=0$, assuming that each vector $v_{j}$ belongs either to $V_{\mathrm{Im}}$ or to $V_{\mathrm{Ker}}$. If the number of the $v_{j}$ 's which belong to $V_{\text {Ker }}$ is not equal to 1 , then all terms $\omega_{g}\left(v_{1}, \ldots, \widehat{v}_{j}, \ldots, v_{2 d(g)+1}\right)$, in the corresponding sum (2.9) vanish by the definition of $\omega_{g}$. So assume $v_{j} \in V_{\text {Ker }}$ is this one vector. Then, we have: $\left[v_{j}, g\right]=v_{j} \cdot g-g \cdot v_{j}=(\operatorname{Id}-g)\left(v_{j}\right) \cdot g=0$, since $v_{j} \in \operatorname{Ker}(\operatorname{Id}-g)$. Hence, for the corresponding term in (2.9) we get: $\left[v_{j}, \psi_{g}\left(v_{1}, \ldots, \widehat{v}_{j}, \ldots, v_{2 d(g)+1}\right)\right]=0$, and (2.10) follows.

Observe next that the algebra structure on $\mathrm{H}_{\kappa}$ induces a natural cup-product map:

$$
\cup: \operatorname{Hom}_{\mathbb{C}}\left(\Lambda^{p} V, \mathrm{H}_{\kappa}\right) \otimes \operatorname{Hom}_{\mathbb{C}}\left(\Lambda^{q} V, \mathrm{H}_{\kappa}\right) \rightarrow \operatorname{Hom}_{\mathbb{C}}\left(\Lambda^{p+q} V, \mathrm{H}_{\kappa} \otimes \mathrm{H}_{\kappa}\right) \stackrel{\text { mult }}{\rightarrow} \operatorname{Hom}_{\mathbb{C}}\left(\Lambda^{p+q} V, \mathrm{H}_{\kappa}\right) \text {. }
$$

An elementary linear algebra shows that for any $g_{1}, g_{2} \in \Gamma$ one has an inequality: $d\left(g_{1}\right)+$ $d\left(g_{2}\right) \geq d\left(g_{1} g_{2}\right)$ and, moreover, in $\operatorname{Hom}_{\mathbb{C}}\left(\Lambda^{\bullet} V, \mathrm{H}_{\kappa}\right)$ we have:

$$
\psi_{g_{1}} \cup \psi_{g_{2}}=\left\{\begin{array}{cccc}
\psi_{g_{1} g_{2}} & \text { if } & d\left(g_{1}\right)+d\left(g_{2}\right)=d\left(g_{1} g_{2}\right) \\
0 & \text { if } & d\left(g_{1}\right)+d\left(g_{2}\right)>d\left(g_{1} g_{2}\right) .
\end{array}\right.
$$

Therefore, the assignment: $g \mapsto \psi_{g}$ extends by $\mathbb{C}$-linearity to an Ad $\Gamma$-equivariant graded algebra homomorphism $\psi^{\kappa}: \operatorname{gr}_{\bullet}^{F} \mathbb{C} \Gamma \rightarrow \operatorname{Hom}_{\mathbb{C}}\left(\Lambda^{\bullet} V, \mathrm{H}_{\kappa}\right)$, where ' $F$ ' is the filtration on $\mathbb{C} \Gamma$ introduced before Theorem 1.8. Equation (2.10) implies that the homomorphism $\psi^{\kappa}$ induces, when restricted to $\Gamma$-invariants, a well-defined graded algebra homomorphism into Hochschild cohomology $\psi_{\mathrm{z}}^{\kappa}: \mathrm{gr}_{\bullet} \mathrm{Z \Gamma} \rightarrow \mathrm{HH}^{\bullet}\left(\mathrm{H}_{\kappa}\right)$.

To complete the proof of part (i) of Theorem 1.8 we use an explicit calculation carried out in [AFLS] of Hochschild cohomology for $A_{t} \# \Gamma$, the smash-product of the Weyl algebra, $\mathrm{A}_{t}=T V / I\langle x \cdot y-y \cdot x-t \cdot \omega(x, y)\rangle_{x, y \in V}$, with $\Gamma$. Specifically, it has been shown in [AFLS] that for Hochschild cohomology one has:

$$
\operatorname{dim} H H^{j}\left(\mathrm{~A}_{t} \# \Gamma\right)= \begin{cases}\mathbf{n}(i) & \text { if } j=2 i \\ 0 & \text { if } j \text { is odd }\end{cases}
$$


where the numbers $\mathbf{n}(i)$ have been defined in (1.9). We note that, for $c=0$ (and any $t \neq 0$ ), we have: $H_{t, 0}=A_{t} \# \Gamma$. Furthermore, it is easy to verify by going through the calculation in [AFLS] that, in the special case: $\kappa=(t, 0)$, the map $\psi_{\mathrm{z}}^{\kappa}$ gives in effect a bijection

$$
\psi_{\mathrm{z}}^{\kappa}: \mathrm{gr}_{\bullet}^{F} \mathrm{Z \Gamma} \stackrel{\sim}{\longrightarrow} H H^{\bullet}\left(\mathrm{A}_{t} \# \Gamma\right)
$$

Now fix $\kappa=(t, c) \in \mathbb{C}^{*} \times \mathbf{C}$, introduce an auxiliary complex parameter $r \in \mathbb{C}$, and consider the family $\left\{\mathrm{H}_{t, r \cdot c}\right\}_{r \in \mathbb{C}}$ as a single $\mathbb{C}[r]$-algebra, $\mathbb{H}$, such that: $\mathbb{H} / r \cdot \mathbb{H}=A_{t} \# \Gamma$. Write $H H^{\bullet}(\mathbb{H})$ for the relative Hochschild cohomology of $\mathbb{H}$ with respect to the subalgebra $\mathbb{C}[r]$. The morphisms $\psi_{\mathrm{z}}^{\kappa}, \kappa=(t, r \cdot c)$, assemble together to give a graded $\mathbb{C}[r]$-algebra morphism:

$$
\psi_{\mathrm{z}}:\left(\mathrm{gr}_{\bullet}^{\mathrm{F}} \mathrm{Z \Gamma}\right)[r] \longrightarrow H H^{\bullet}(\mathbb{H})
$$

The algebra $\mathbb{H}$ is flat over $\mathbb{C}[r]$, due to the PBW-property. The canonical filtration on the algebra $\mathrm{H}_{t, c}$ makes $\mathbb{H}$ a filtered $\mathbb{C}[r]$-algebra whose degree zero component is $\mathbb{C} \Gamma[r]$. This filtration induces a filtration on a relative version of Koszul complex (2.8) used for computing $H H^{\bullet}(\mathbb{H})$, the relative Hochschild cohomology. This way the relative Koszul complex is filtered by an increasing sequence of subcomplexes formed by free $\mathbb{C}[r]$-modules of finite rank. Therefore, since $H H^{\text {odd }}\left(\mathrm{A}_{t} \# \Gamma\right)=0$, the Euler-Poincaré principle and semi-continuity imply that, for any $i$, the $\mathbb{C}[r]$-module $H H^{2 i+1}(\mathbb{H})$ is supported at most on a countable subset of $\mathbb{C}$ and, similarly, $H H^{2 i}(\mathbb{H})$ is, up to torsion on a countable subset of $\mathbb{C}$, a free $\mathbb{C}[r]$-module of rank: $\operatorname{dim} H H^{2 i}\left(\mathrm{~A}_{t} \# \Gamma\right)=\mathbf{n}(i)$. Further, since the Koszul complex for $\mathbb{H}$ has finite length, there are only finitely many non-zero cohomology groups involved. Thus, the map (2.14) is a map between $\mathbb{C}[r]$-modules of the same generic rank. Moreover, both modules have no torsion at $r=0$, and at this point the map specializes to a bijection. It follows that there is at most a countable set BAD $\subset \mathbb{C}$ such that for any $r \in \mathbb{C} \backslash \mathrm{BAD}$, the map (2.14) specializes to a bijection, hence an algebra isomorphism: $\psi_{\mathrm{z}}^{t, r \cdot c}:\left(\mathrm{gr}_{\bullet}^{F} \mathrm{Z} \Gamma\right) \stackrel{\sim}{\longrightarrow} H H^{\bullet}\left(\mathrm{H}_{t, r \cdot c}\right)$. This proves part (i) of Theorem 1.8, since $\mathrm{H}_{t, r \cdot c} \simeq \mathrm{H}_{t / r, c}$.

Recall the 'averaging' idempotent $\mathbf{e}=\frac{1}{\Gamma \mid} \sum_{g \in \Gamma} g \in \mathbb{C} \Gamma$. If the group $\Gamma$ acts on an associative algebra $\mathcal{A}$ by algebra automorphisms, then one has a canonical algebra isomorphism:

$$
\mathcal{A}^{\Gamma} \stackrel{\sim}{\longrightarrow} \mathbf{e} \cdot(\mathcal{A} \# \Gamma) \cdot \mathbf{e} \quad, \quad a \mapsto a \cdot \mathbf{e}=\mathbf{e} \cdot a
$$

Recall now the spherical subalgebra: $\mathbf{e H}_{t, c} \mathbf{e} \subset \mathrm{H}_{t, c}$ (we warn the reader that this subalgebra does not contain the unit of $\mathrm{H}_{\kappa}$ ). For $t=0$ and $c=0$, from (2.4) and (2.15) one finds: $\mathbf{e H}_{0,0} \mathbf{e}=\mathbf{e}(S V \# \Gamma) \mathbf{e} \simeq(S V)^{\Gamma}$, the algebra of $\Gamma$-invariants. Furthermore, isomorphism (2.4) yields readily: $\operatorname{gr}\left(\mathbf{e H}_{t, c} \mathbf{e}\right) \simeq(S V)^{\Gamma}$. Similarly, as we have noticed in the proof of Theorem 1.8, for $c=0$ and any $t \neq 0$, we have: $\mathrm{H}_{t, 0}=\mathrm{A}_{t} \# \Gamma$, where $\mathrm{A}_{t}=T V / I\langle x \cdot y-y \cdot x-t \cdot \omega(x, y)\rangle_{x, y \in V}$, is the Weyl algebra of the symplectic vector space $(V, t \cdot \omega)$. By (2.15), the spherical subalgebra $\mathbf{e H}_{t, 0} \mathbf{e}$ is isomorphic to $\mathrm{A}_{t}^{\Gamma}$, the subalgebra of $\Gamma$-invariants in the Weyl algebra. 
Theorem 2.16 For any fixed $t \neq 0$, the family $\left\{\mathrm{H}_{t, c}\right\}_{c \in \mathrm{C}}$ gives a universal deformation of the algebra $\mathrm{A}_{t} \# \Gamma$, and the family $\left\{\mathbf{e H}_{t, c} \mathbf{e}\right\}_{c \in \mathrm{C}}$ gives a universal deformation of the algebra $\mathrm{A}_{t}^{\Gamma}$.

To prove the theorem, we fix $t \neq 0$ and given $c \in \mathrm{C}$, treat the family $\left\{\mathrm{H}_{t, \varepsilon \cdot c}\right\}_{\varepsilon \in \mathbb{C}}$ as a single $\mathbb{C}[\varepsilon]$-algebra, still denoted $\mathrm{H}_{t, \varepsilon c}$, where $\varepsilon$ is now regarded as an indeterminate.

Lemma 2.17 For any nonzero $c \in \mathrm{C}$, the $\mathbb{C}[\varepsilon] /\left(\varepsilon^{2}\right)$-algebras: $\mathrm{H}_{t, \varepsilon c} / \varepsilon^{2} \mathrm{H}_{t, \varepsilon c}$ and $\mathrm{H}_{t, 0} \bigotimes_{\mathbb{C}}\left(\mathbb{C}[\varepsilon] / \varepsilon^{2}\right)$ are not isomorphic.

Proof. Observe first that we have a natural isomorphism of $\mathbb{C}$-vector spaces $\phi$ : $\mathrm{H}_{t, 0} \stackrel{\sim}{\longrightarrow} \varepsilon \cdot \mathrm{H}_{t, \varepsilon c} / \varepsilon^{2} \cdot \mathrm{H}_{t, \varepsilon c}$. Assume, contrary to the claim, that there is an isomorphism $\mathrm{H}_{t, \varepsilon c} / \varepsilon^{2} \cdot \mathrm{H}_{t, \varepsilon c} \simeq \mathrm{H}_{t, 0} \bigotimes_{\mathbb{C}}\left(\mathbb{C}[\varepsilon] / \varepsilon^{2}\right)$, as algebras over $\mathbb{C}[\varepsilon] /\left(\varepsilon^{2}\right)$, the ring of 'dual numbers'. Then the natural imbedding: $A_{t} \hookrightarrow A_{t} \# \Gamma=\mathrm{H}_{t, 0}$ lifts to the dual numbers, that is, one can find a $\mathbb{C}$-linear map $f: V \rightarrow \mathrm{H}_{t, 0}$ such that in $\mathrm{H}_{t, \varepsilon c} / \varepsilon^{2} \cdot \mathrm{H}_{t, \varepsilon c}$, the following equation holds:

$(x+\phi \circ f(x)) \cdot(y+\phi \circ f(y))-(y+\phi \circ f(y)) \cdot(x+\phi \circ f(x))=t \cdot \omega(x, y) \cdot 1 \quad, \quad \forall x, y \in V$

Using the defining relations in $\mathrm{H}_{t, \varepsilon c}$ and applying the isomorphism $\phi^{-1}$, this gives the following equation in $\mathrm{H}_{t, 0}$ :

$$
[f(x), y]+[x, f(y)]+\sum_{s} c_{s} \cdot \omega_{s}(x, y) \cdot s=0
$$

Write $f(x)=\sum_{g \in \Gamma} f_{g}(x) \cdot g$, where $f_{g}(x) \in \mathrm{A}_{t}$. Fix $s \in S$ such that $c_{s} \neq 0$. Taking the $s$-term of the equation (2.18), we get

$$
\left[f_{s}(x), y\right]+f_{s}(x) \cdot\left(y^{s}-y\right)+\left[x, f_{s}(y)\right]+f_{s}(y) \cdot\left(x-x^{s}\right)=-c_{s} \cdot \omega_{s}(x, y) .
$$

One may choose $x, y$, a basis of the 2-dimensional vector space $\operatorname{Im}(s-\operatorname{Id})$ such that $\omega_{s}(x, y)=1$, and such that $s(x)=\lambda x, s(y)=\lambda^{-1} y$, where $\lambda \neq 1$. Put $a:=\frac{1}{c_{s}} f_{s}(x) \neq 0$, and $b:=\frac{1}{c_{s}} f_{s}(y) \neq 0$. From equation (2.19) we find

$$
[a, y]-\left(1-\lambda^{-1}\right) a \cdot y+[x, b]+(1-\lambda) b \cdot x=-1 \text {. }
$$

Let $A^{\text {Ker }}$, resp. $A^{I m}$, denote the subalgebra in $A_{t}$ generated by the subspace $\operatorname{Ker}(\operatorname{Id}-s) \subset$ $V$, resp. $\operatorname{Im}(\operatorname{Id}-s)$. Thus, $\mathrm{A}_{t}=\mathrm{A}^{\mathrm{Ker}} \otimes \mathrm{A}^{\mathrm{Im}}$, and the monomials: $\left\{x^{m} y^{n}\right\}_{m, n \geq 0}$ form a $\mathbb{C}$-basis of $\mathrm{A}^{\mathrm{Im}}$. Write $a, b$ as a $\mathrm{A}^{\mathrm{Ker}}$-linear combination of the monomials $x^{m} y^{n}$. Then begin treating $x$ and $y$ as commuting variables, so that the expressions for $a, b$ become certain polynomials: $a, b \in \mathrm{A}^{\mathrm{Ker}}[x, y]$. Using the identity: $b x=-[x, b]+x b$, and setting $t=1$, from equation (2.20) we deduce

$$
\left(\frac{\partial}{\partial x}-\left(1-\lambda^{-1}\right) \cdot y\right) a+\left(\lambda \frac{\partial}{\partial y}+(1-\lambda) \cdot x\right) b=-1 \quad \text { in } \quad \mathrm{A}^{\mathrm{Ker}}[x, y] .
$$


Set $A:=-a, B:=-\lambda b$, and $\nu:=1-\lambda^{-1}$. Our equation reads:

$$
\left(\frac{\partial}{\partial x}-\nu \cdot y\right) A+\left(\frac{\partial}{\partial y}-\nu \cdot x\right) B=1 \quad, \quad A, B \in \mathrm{A}^{\mathrm{Ker}}[x, y] .
$$

The proof of the Lemma is now completed by the following

Claim: For any $\nu \in \mathbb{C}^{*}$, equation (2.21) has no polynomial solutions $A, B \in \mathrm{A}^{\mathrm{Ker}}[x, y]$.

Proof of Claim. Clearly, it suffices to prove that equation (2.21) has no solutions $A, B \in$ $\mathbb{C}[x, y]$. Further, replacing the function $A$, by the function: $(x, y) \mapsto \nu^{-1} \cdot A(\nu \cdot x, \nu \cdot y)$, and making a similar substitution for $B$, one reduces the problem to the case: $\nu=1$. Assuming this, we further specialize: $x=z, y=\bar{z}$, where $z$ is a complex variable, and $\bar{z}$ its complex conjugate. Integrating both sides of equation (2.21) for $\nu=1$ against the Gaussian measure $e^{-z \bar{z}} d z d \bar{z}$, we get

$$
\int_{\mathbb{C}}\left(\left(\frac{\partial}{\partial z}-\bar{z}\right) A(z, \bar{z})+\left(\frac{\partial}{\partial \bar{z}}-z\right) B(z, \bar{z})\right) e^{-z \bar{z}} d z d \bar{z}=\int_{\mathbb{C}} e^{-z \bar{z}} d z d \bar{z}
$$

where the integral on the LHS is absolutely convergent, for any polynomials $A, B$. But, integration by parts shows that the LHS $2.22=0$, while clearly RHS 2.22 $>0$. The contradiction completes the proof.

Proof of Theorem 2.16: Deformations of the algebra $A_{t} \# \Gamma$ are controlled by $H H^{2}\left(A_{t} \# \Gamma\right)$, the second Hochschild cohomology group of $A_{t} \# \Gamma$, and obstructions to deformations are controlled by the third Hochschild cohomology group of $A_{t} \# \Gamma$. It was shown in [AFLS] that $\operatorname{dim} H H^{2}\left(\mathrm{~A}_{t} \# \Gamma\right)=$ number of $\mathrm{Ad} \Gamma$-conjugacy classes in $S$, and $H H^{3}\left(\mathrm{~A}_{t} \# \Gamma\right)=0$. It follows that the deformations are unobstructed, the point $A_{t} \# \Gamma$ is a smooth point of the moduli space of all deformations, and the tangent space at the point $A_{t} \# \Gamma$ to the moduli space has dimension equal to $\operatorname{dim} C$. The cohomology classes corresponding to deformations from the family $\left\{\mathrm{H}_{t, c}\right\}_{c \in C}$ form a certain vector subspace $H \subset H^{2}\left(\mathrm{~A}_{t} \# \Gamma\right)$. Clearly, $\operatorname{dim} H=\operatorname{dim} \mathrm{C}$ if and only if, for any non-zero $c \in \mathrm{C}$, the algebra $\mathrm{H}_{t, \varepsilon c}$ gives a non-trivial deformation of $\mathrm{A}_{t} \# \Gamma$ over $\mathbb{C}[\varepsilon] /\left(\varepsilon^{2}\right)$. Hence, Lemma 2.17 implies: $\operatorname{dim} H=$ $\operatorname{dim} \mathrm{C}=\operatorname{dim} H H^{2}\left(\mathrm{~A}_{t} \# \Gamma\right)$, and therefore $H=H H^{2}\left(\mathrm{~A}_{t} \# \Gamma\right)$. Thus, our family is a universal family.

To prove the second claim observe that by (2.15) we have: $\mathbf{e}\left(A_{t} \# \Gamma\right) \mathbf{e} \simeq A_{t}^{\Gamma}$. Hence the family: $\left\{\mathbf{e H}_{t, c} \mathbf{e}\right\}_{c \in C}$ gives a flat deformation of the algebra $A_{t}^{\Gamma}$. The rest of the proof is entirely similar: Morita equivalence of the algebras $A_{t} \# \Gamma$ and $A_{t}^{\Gamma}$ implies that these algebras have isomorphic Hochschild cohomology, see [AFLS].

Observe that the family $\left\{\mathcal{A}_{t}\right\}_{t \in \mathbb{C}}$, where $\mathcal{A}_{t}:=\mathrm{A}_{t}^{\Gamma}$ if $t \neq 0$, and $\mathcal{A}_{t=0}:=(S V)^{\Gamma}$, is flat, hence, gives a deformation of the algebra $\mathcal{A}_{t=0}=(S V)^{\Gamma}$. Since the latter algebra is commutative, the general construction of $\S 15$ yields a Poisson structure on the algebra $(S V)^{\Gamma}=\mathbb{C}\left[V^{*}\right]^{\Gamma}$. It is well-known and easy to verify that the corresponding Poisson bracket $\{-,-\}_{\omega}$ is obtained by restricting the Poisson bracket on $\mathbb{C}\left[V^{*}\right]$ induced by the symplectic structure on $V^{*}$. Note that, for the standard grading $(S V)^{\Gamma}=\bigoplus_{i \geq 0}\left(S^{i} V\right)^{\Gamma}$, we have $\{-,-\}_{\omega}:\left(S^{i} V\right)^{\Gamma} \times\left(S^{j} V\right)^{\Gamma} \longrightarrow\left(S^{i+j-2} V\right)^{\Gamma}$. More generally, a Poisson bracket $B$ on $(S V)^{\Gamma}$ is said to be of degree $\ell$ if, for any $i, j \geq 0$, we have $B:\left(S^{i} V\right)^{\Gamma} \times\left(S^{j} V\right)^{\Gamma} \longrightarrow$ $\left(S^{i+j+\ell} V\right)^{\Gamma}$. 
Lemma 2.23 (i) Any Poisson bracket on $(S V)^{\Gamma}$ of degree $(-2)$ is proportional to $\{-,-\}_{\omega}$.

(ii) Any Poisson bracket on $(S V)^{\Gamma}$ of degree $\ell<-2$ is zero.

Proof. We identify $V$ with $V^{*}$ using the symplectic form. Let $Y$ be the set of points of $V$ with a nontrivial isotropy group in $\Gamma$. Then $(V \backslash Y) / \Gamma$ is a smooth manifold. A Poisson bracket on $(S V)^{\Gamma}$ in particular defines a Poisson bracket on $(V \backslash Y) / \Gamma$, which is the same as a $\Gamma$-invariant Poisson bracket, that is a bivector field, on $V \backslash Y$.

Since $\Gamma \subset S p(V)$ is a finite group, any nontrivial element of $\Gamma$ has at least two eigenvalues $\neq 1$. This implies that the codimension of $Y$ is at least 2. Thus, by Hartogs theorem, any regular bivector on $V \backslash Y$ extends by continuity to a regular algebraic bivector on the whole of $V$. But a regular bivector on $V$ of homogeneity degree $\ell$ must vanish if $\ell<-2$, and comes from an element of $\Lambda^{2} V$ if $\ell=-2$. In the latter case, since our bracket is $\Gamma$-invariant, by indecomposability of $(V, \omega, \Gamma)$, this bracket must be a multiple of $\{-,-\}_{\omega}$.

Lemma 2.24 Let $t, c$ be such that the algebra $\mathrm{H}_{t, c}$ is commutative. Let $\chi: \mathbf{e H}_{t, c} \mathbf{e} \rightarrow \mathbb{C}$ be a generic character, and let $T_{\chi}=\mathrm{H}_{t, c} \mathbf{e} \otimes_{\mathbf{e}} \mathrm{H}_{t, c} \mathbf{e} \chi$ be the corresponding induced $\mathrm{H}_{t, c}$-module. Then $T_{\chi}$ is isomorphic, as a $\Gamma$-module, to the regular representation of $\Gamma$.

Proof. Consider the family of algebras $\left\{\mathrm{H}_{r t, r c}\right\}_{r \in \mathbb{C}}$. These algebras are isomorphic to each other for all $r \neq 0$, so it is sufficient to prove the lemma for a generic member of the family. To this end, we treat the family $\left\{\mathrm{H}_{r t, r c}\right\}_{r \in \mathbb{C}}$ as a single $\mathbb{C}[r]$-algebra $\widetilde{\mathrm{H}}$. This algebra is flat over $\mathbb{C}[r]$, due to PBW-property. It follows that $\mathbf{e} \widetilde{H} \mathbf{e}$ is a commutative flat $\mathbb{C}[r]$-algebra.

Further, $\widetilde{H e}$ is a finitely generated e $\widetilde{H}$-module, due to Theorem 1.5(ii) to be proved below (which is independent of the material in between). Fix a simple $\Gamma$-module $E$, and set $F=\operatorname{Hom}_{\Gamma}(E, \widetilde{H} \mathbf{e})$. By standard results in invariant theory, see proof of Theorem 1.5 below, $F$ is a finitely generated e $\widetilde{H e}$-module which is, moreover, flat over over $\mathbb{C}[r]$, by the PBW-property. We write $F_{r}=F / r \cdot F=\operatorname{Hom}_{\Gamma}\left(E, \mathrm{H}_{r t, r c} \mathbf{e}\right)$ for the corresponding $\mathbf{e H}_{r t, r c} \mathbf{e}$-module.

Given a commutative $\mathbb{C}$-algebra $\mathcal{A}$ without zero-divisors, and a $\mathcal{A}$-module $M$, let $\mathrm{rk}_{\mathcal{A}}(M)$ denote the generic rank of $M$, i.e., $\operatorname{rk}_{\mathcal{A}}(M):=\operatorname{dim}_{Q(\mathcal{A})}\left(Q(\mathcal{A}) \otimes_{\mathcal{A}} M\right)$, where $Q(\mathcal{A})$ stands for the field of fractions of $\mathcal{A}$. It is clear that $\mathrm{rk}_{\mathrm{eH}_{0,0} \mathrm{e}}\left(F_{0}\right)=\operatorname{dim} E$. On the other hand, proving the lemma amounts to showing that $\mathrm{rk}_{\mathrm{e} \tilde{\mathrm{e}}} F=\operatorname{dim} E$.

To this end, let $\mathfrak{p}=(\mathbf{e} \widetilde{H} \mathbf{e}) \cdot r \subset \mathbf{e} \widetilde{H} \mathbf{e}$ be the prime ideal generated by $r$, and set $\mathcal{A}:=(\mathbf{e} \widetilde{\mathbf{e}})_{(\mathfrak{p})}$, the localization of $\mathbf{e} \widetilde{\mathrm{H}} \mathbf{e}$ with respect to $\mathfrak{p}$. Thus, $\mathcal{A}$ is a local ring with the residue class field $\mathcal{A}_{0}:=\mathcal{A} / \mathcal{A} \cdot r \simeq Q\left(\mathbf{e H}_{0,0} \mathbf{e}\right)$, the field of fractions of the algebra $\mathbf{e H}_{0,0} \mathbf{e}$. Furthermore, $\mathcal{A}$ is a local ring of dimension 1 with local parameter $r$, due to the $\mathbb{C}[r]$-flatness of e $\widetilde{H}$. Let $\mathcal{F}=\mathcal{A} \otimes_{\text {e } \tilde{H} \mathbf{e}} F$ denote the $\mathcal{A}$-module obtained by localizing at $\mathfrak{p}$ the e $\widetilde{H}$-module $F$, and set $\mathcal{F}_{0}:=\mathcal{F} / \mathcal{F} \cdot r$, an $\mathcal{A}_{0}$-module. Observe that since $F$ is $\mathbb{C}[r]$-flat, and $r$ is a local parameter on $\mathcal{A}$, the module $\mathcal{F}$ is free over $\mathcal{A}$. Thus, we obtain

$$
\mathrm{rk}_{\mathrm{e} \tilde{\mathrm{He}}} F=\mathrm{rk}_{\mathcal{A}} \mathcal{F}=\mathrm{rk}_{\mathcal{A}_{0}}\left(\mathcal{F}_{0}\right)=\mathrm{rk}_{\mathrm{eH}_{0,0} \mathrm{e}}\left(F_{0}\right) .
$$


Proof of Theorem 1.6. Introduce an auxiliary variable ' $\hbar$ ', and set $T V[\hbar]=T V \otimes \mathbb{C}[\hbar]$. We treat $T V[\hbar]$ as a graded algebra with $\operatorname{deg} \hbar=2$, and assume that the group $\Gamma$ acts trivially on $\hbar$. Let

$$
\widetilde{\mathrm{H}}:=(T V[\hbar] \# \Gamma) / I\left\langle x \otimes y-y \otimes x-\kappa(x, y) \cdot \hbar \in T^{2} V \oplus \mathbb{C} \Gamma[\hbar]\right\rangle_{x, y \in V},
$$

be a 'homogenized' version of the algebra $\mathrm{H}_{\kappa}$. We set $\mathcal{A}=\mathbf{e} \widetilde{H} \mathbf{e}$, the corresponding spherical subalgebra. Clearly, $\mathcal{A}$ is a flat $\mathbb{C}[\hbar]$-algebra such that: $\mathcal{A} / \hbar \mathcal{A}=\operatorname{gr}\left(\mathbf{e H}_{t, c} \mathbf{e}\right)$. Moreover, since $\mathcal{A} /(\hbar-1) \mathcal{A}=\mathbf{e H}_{t, c} \mathbf{e}$, one may view the algebra $\mathbf{e H}_{t, c} \mathbf{e}$ as a flat $\hbar$-deformation of the commutative algebra $\operatorname{gr}\left(\mathbf{e H}_{t, c} \mathbf{e}\right)=(S V)^{\Gamma}$. Thus, for any $(t, c)$, by the general construction of $\S 15$, this deformation gives rise to a Poisson bracket $B_{t, c}$ on $(S V)^{\Gamma}$. Let $\mathbf{m}_{t, c} \in\{1,2, \ldots, \infty\}$, be the number involved in the construction of the Poisson bracket, see Lemma 15.1. We have:

Claim 2.25 (i) There are only two alternatives: either $\mathbf{m}_{t, c}=1$, or $\mathbf{m}_{t, c}=\infty$. The algebra $\mathbf{e H}_{t, c} \mathbf{e}$ is non-commutative if $\mathbf{m}_{t, c}=1$, and commutative if $\mathbf{m}_{t, c}=\infty$.

(ii) If $\mathbf{m}_{t, c}=1$, then $B_{t, c}=f(t, c) \cdot\{-,-\}_{\omega}$, where $f: \mathbb{C} \oplus \mathbb{C} \rightarrow \mathbb{C}$ is a non-zero linear function.

To prove part (i) of the Claim, note that since $\operatorname{deg} \hbar=2$, it is clear that the Poisson bracket $B_{t, c}$ has degree $\left(-2 \mathbf{m}_{t, c}\right)$. Thus, by Lemma 2.23(ii), in order for $B_{t, c}$ to be non-zero we must have $\mathbf{m}_{t, c}=1$. But, by Lemma 15.1, vanishing of $B_{t, c}$ implies commutativity of the algebra $\mathcal{A}$, and (i) follows.

To prove part (ii) of Claim 2.25, we change our point of view, and regard $t$ and $c$ as variables, and assign them grade degree 2 , while set $\operatorname{deg} \hbar=0$. Thus, $\mathcal{A}$ becomes now a graded $\mathbb{C}[\mathbb{C} \oplus \mathbb{C}]$-algebra, depending on $\hbar$, the deformation parameter. Applying the Poisson bracket construction of $\S 15$ in this new setting we get, for $\mathbf{m}=1$, a bracket $B$ on $(S V)^{\Gamma} \otimes \mathbb{C}[\mathbb{C} \oplus \mathbb{C}]$ of degree $(-2)$. By Lemma 2.23(i), we have: $B=f(t, c)\{-,-\}_{\omega}$, for some $f(t, c) \in \mathbb{C}$. Since the relations in $\mathrm{H}_{t, c}$ become homogeneous in our new grading: $\operatorname{deg}(t)=\operatorname{deg}(c)=2$, it follows that: $t, c \mapsto f(t, c)$ is a certain linear function on $\mathbb{C} \oplus \mathbf{C}$. Moreover, for any $(t, c) \in \mathbb{C} \oplus \mathrm{C}$ such that $B_{t, c} \neq 0$, the Poisson bracket $B_{t, c}$ is clearly a specialisation of $B$ at the point $(t, c)$, that is: $B_{t, c}=f(t, c)\{-,-\}_{\omega}$. This completes the proof of Claim 2.25(ii).

Claim 2.25 implies that the algebra $\mathbf{e H}_{t, c} \mathbf{e}$ is commutative if and only if $f(t, c)=0$. We see that the parameters $(t, c)$ such that $\mathbf{e H}_{t, c} \mathbf{e}$ is commutative form a hyper-plane in $\mathbb{C} \oplus \mathrm{C}$ given by the equation: $f(t, c)=0$. Therefore, to complete the proof of the theorem, it suffices to show that $f(t, c)$ is a multiple of $t$, i.e. that the above hyper-plane is the one given by the equation $t=0$. To prove this assume that, for some $t, c$, one has $f(t, c)=0$, and hence the algebra $\mathbf{e H}_{t, c} \mathbf{e}$ is commutative. In this case, choose a generic character $\chi: \mathbf{e H}_{t, c} \mathbf{e} \rightarrow \mathbb{C}$, and let $T_{\chi}=\mathrm{H}_{t, c} \otimes_{\mathbf{e}} \mathrm{H}_{t, c} \mathbf{e} \chi$ be the corresponding induced $\mathrm{H}_{t, c}$-module. By lemma 2.24, this module is isomorphic, as a $\Gamma$ module, to the regular representation of $\Gamma$.

Now, for any $g \in \Gamma$ such that $g \neq 1$, in the regular $\Gamma$-representation one has $\operatorname{tr}(g)=0$. Taking the traces in $T_{\chi}$ of both sides of the main commutation relation: $x \otimes y-y \otimes x=$ 
$\kappa(x, y)$ in the algebra $\mathrm{H}_{t, c}$ we deduce: $t=0$. Thus, the two hyperplanes: $f(t, c)=0$, and $t=0$ coincide, and we are done.

According to Theorem 1.6, the algebra $\mathbf{e H}_{0, c} \mathbf{e}$ is commutative for any $c \in \mathrm{C}$, and the family $\left\{\mathbf{e H}_{t, c} \mathbf{e}\right\}_{t \in \mathbb{C}}$ gives, by the PBW-theorem, a flat deformation of $\mathbf{e H}_{0, c} \mathbf{e}$. Hence, by the general construction of $\S 15$ this deformation induces a Poisson bracket on $\mathbf{e H}_{0, c} \mathbf{e}$, to be denoted $\{-,-\}$. Recall further that the algebra $\mathbf{e H}_{0, c} \mathbf{e}$ has a natural increasing filtration $F_{\bullet}\left(\mathbf{e H}_{0, c} \mathbf{e}\right)$ induced from that on $\mathrm{H}_{0, c}$.

Let $B$ be any Poisson bracket on $\mathbf{e H}_{0, c} \mathbf{e}$. We say that $B$ has filtration degree $\ell \in \mathbb{Z}$, if the following two conditions hold:

- $B\left(F_{i}\left(\mathbf{e H}_{0, c} \mathbf{e}\right), F_{j}\left(\mathbf{e H}_{0, c} \mathbf{e}\right)\right) \subset F_{i+j+\ell}\left(\mathbf{e H}_{0, c} \mathbf{e}\right) \quad, \quad \forall i, j \geq 0$;

- There exist $i, j \geq 0$, such that: $B\left(F_{i}\left(\mathbf{e H}_{0, c} \mathbf{e}\right), F_{j}\left(\mathbf{e H}_{0, c} \mathbf{e}\right)\right) \not \subset F_{i+j+\ell-1}\left(\mathbf{e H}_{0, c} \mathbf{e}\right)$.

Lemma 2.26 Let $B$ be a non-zero Poisson bracket on $\mathbf{e H}_{0, c} \mathbf{e}$ of filtration degree $\ell \leq-2$. Then $\ell=-2$ and, moreover, $B=\lambda \cdot\{-,-\}$, for some non-zero constant $\lambda \in \mathbb{C}$.

Proof. The Poisson bracket $B$ induces naturally a non-zero Poisson bracket gr $B$ on $\operatorname{gr}\left(\mathbf{e H}_{0, c} \mathbf{e}\right)$ of degree $\ell$. Recall that $\operatorname{gr}\left(\mathbf{e H}_{0, c} \mathbf{e}\right) \simeq(S V)^{\Gamma}$. Thus, we may view gr $B$ as a nonzero Poisson bracket on $(S V)^{\Gamma}$. Hence, Lemma 2.23(ii) yields: $\ell=-2$. Moreover, part (i) of the same Lemma implies that there exists a constant $\lambda \in \mathbb{C}$ such that gr $B=\lambda \cdot\{-,-\}_{\omega}$. It follows that $B-\lambda \cdot\{-,-\}$ is a Poisson bracket on $\mathbf{e H}_{0, c} \mathbf{e}$ of filtration degree $<\ell=-2$. The argument above shows that this bracket must vanish, and we are done.

\section{Representation theory of the algebra $\mathrm{H}_{0, c}$}

Proof of Theorem 1.5: It is known from invariant theory that $(S V)^{\Gamma}$ is a finitely generated algebra and, moreover, $S V$ is a finitely generated $(S V)^{\Gamma}$-module: by Hilbert's Basis theorem, each isotypic component of $S V$ is finitely generated over $(S V)^{\Gamma}$, see e.g. $[\mathrm{PV}]$. Hence, the isomorphisms: $\operatorname{gr}\left(\mathbf{e H}_{t, c} \mathbf{e}\right) \simeq(S V)^{\Gamma}$ and $\operatorname{gr}\left(\mathrm{H}_{t, c} \mathbf{e}\right) \simeq S V$ insured by Theorem 1.3 imply that $\mathbf{e H}_{t, c} \mathbf{e}$ is a finitely generated algebra without zero divisors, and $\mathrm{H}_{t, c} \mathbf{e}$ is a finitely generated $\mathbf{e H}_{t, c} \mathbf{e}$-module.

Next we need to recall some definitions. Given a not necessarily commutative associative $\mathbb{C}$-algebra $\mathcal{A}$, one defines the notion of a rigid Auslander dualizing complex for $\mathcal{A}$, see $[\mathrm{VB}],[\mathrm{YZ}]$. This is an object $\mathbb{D}_{\mathcal{A}} \in D_{f}(\mathcal{A}$-bimod $)=$ bounded derived category of complexes of $\mathcal{A}$-bimodules, whose cohomology groups are finitely generated both as left and as right $\mathcal{A}$-modules. Such a dualizing complex might or might not exist, in general, but if it exists it is unique [VB], and enjoys nice functorial properties, as explained in [YZ]. Furthermore, it has been shown in [VB], [YZ] that $\mathbb{D}_{\mathcal{A}}$ does exist if $\mathcal{A}$ is a positively filtered $\mathbb{C}$-algebra, such that $\operatorname{gr}(\mathcal{A})$ is a finitely generated commutative algebra with $\operatorname{gr}_{0}(\mathcal{A})=\mathbb{C}$. In particular, for a commutative finitely generated algebra $\mathcal{A}$, the dualizing complex, $\mathbb{D}_{\mathcal{A}}$, exists and coincides, due to the uniqueness property, with the one known in Commutative Algebra. 
Fix an algebra $\mathcal{A}$ that has the rigid Auslander dualizing complex, $\mathbb{D}_{\mathcal{A}}$. We say that $\mathcal{A}$ is Cohen-Macaulay if all the cohomology groups $H^{i}\left(\mathbb{D}_{\mathcal{A}}\right)$ vanish, except for $i=0$. In the situation of a filtered algebra $\mathcal{A}$, as above, our definition agrees, by [YZ, Prop. 6.18], with other definitions of (non-commutative) Cohen-Macaulay rings that involve GelfandKirillov dimensions, e.g., those used in $[\mathrm{Bj}]$ and $[\mathrm{CBH}]$. For a Cohen-Macaulay algebra $\mathcal{A}$, we will abuse the notation, and write $\mathbb{D}_{\mathcal{A}}$ for the bimodule $H^{0}\left(\mathbb{D}_{\mathcal{A}}\right)$, quasi-isomorphic to it. A Cohen-Macaulay algebra $\mathcal{A}$ is called Gorenstein if $\mathbb{D}_{\mathcal{A}}=\mathcal{A}$. Further, a finitely generated (left) module $M$ over a Cohen-Macaulay algebra $\mathcal{A}$ is said to be a Cohen-Macaulay module of degree $d$ if, for all $i \neq d$ we have: $\operatorname{Ext}_{\mathcal{A} \text {-left }}^{i}\left(M, \mathbb{D}_{\mathcal{A}}\right)=0$. For commutative algebras, all these definitions agree with the standard ones.

The commutative algebra $\operatorname{gr}\left(\mathbf{e H}_{t, c} \mathbf{e}\right) \simeq(S V)^{\Gamma}$ is known to be Gorenstein [Wat]. Part (i) of the Theorem now follows from a result proved by Bjork [Bj] saying that: for a positively filtered associative $\mathbb{C}$-algebra $\mathcal{A}$, with $\operatorname{gr}_{0}(\mathcal{A})=\mathbb{C}$, one has

$$
\operatorname{gr} \mathcal{A} \text { is }\left\{\begin{array}{l}
\text { Cohen-Macaulay, } \\
\text { resp. Gorenstein }
\end{array}\right\} \Longrightarrow \mathcal{A} \text { is }\left\{\begin{array}{l}
\text { Cohen-Macaulay, } \\
\text { resp. Gorenstein }
\end{array}\right\} .
$$

(For the Gorenstein property, Bjork actually proves the implication above with $\mathbb{D}_{\mathcal{A}}$ being replaced by $\mathcal{A}$. But, if $\operatorname{gr}(\mathcal{A})$ is Gorenstein, then an explicit description of $\mathbb{D}_{\mathcal{A}}$ given in [VB, Prop. 8.2, Prop. 8.4] shows that $\mathbb{D}_{\mathcal{A}}=\mathcal{A}$ in this case). This proves part (i).

To prove part (ii) of the Theorem, recall the following standard result from commutative algebra:

Claim ([BBG, Theorem 2.1]). Let $A \subset B$ be finitely generated commutative $\mathbb{C}$-algebras with unit, and $M$ a B-module which is finitely generated over $A$. Then $M$ is CohenMacaulay over $B$ if and only if it is Cohen-Macaulay over $A$.

This implies that $S V$ is a Cohen-Macaulay $(S V)^{\Gamma}$-module of degree 0 (i.e. its support has full dimension). Further, Theorem 1.3 shows that $\operatorname{gr}\left(\mathrm{H}_{\kappa} \mathbf{e}\right) \simeq S V$. We can now apply $[\mathrm{Bj}]$ to $\mathcal{A}=\mathbf{e H}_{\kappa} \mathbf{e}$ and $M=\mathrm{H}_{\kappa} \mathbf{e}$, to conclude that for any $i \geq 0: \operatorname{Ext}_{\operatorname{gr}(\mathcal{A})}^{i}(\operatorname{gr}(M), \operatorname{gr}(\mathcal{A}))=0$ implies: $\operatorname{Ext}_{\mathcal{A}}^{i}(M, \mathcal{A})=0$. It follows, due to the equality $\mathbb{D}_{\mathcal{A}}=\mathcal{A}$, that $\mathrm{H}_{\kappa} \mathbf{e}$ is a CohenMacaulay $\mathbf{e H}_{\kappa} \mathbf{e}$-module.

We now prove (vi). Equip $M=\mathrm{H}_{\kappa} \mathbf{e}$ with the induced increasing filtration $F_{0} M \subset$ $F_{1} M \subset \ldots$, and choose a finite set, $u_{1}, \ldots, u_{\ell}$, of homogeneous generators of the $\operatorname{gr}\left(\mathbf{e} \mathrm{H}_{\kappa} \mathbf{e}\right)-$ module $\operatorname{gr}(M)=\operatorname{gr}\left(\mathrm{H}_{\kappa} \mathbf{e}\right)$. Let $d_{i}=\operatorname{deg}\left(u_{i}\right)$, and let $\widetilde{u}_{i} \in F_{d_{i}} M$ be a representative of $u_{i}$. Then $\widetilde{u}_{1}, \ldots, \widetilde{u}_{\ell}$ generate $M$ as a $\mathbf{e H}_{\kappa} \mathbf{e}$-module. Given an $\mathbf{e} \mathbf{H}_{\kappa} \mathbf{e}$-linear map $f: M \rightarrow M$, we may find an integer $m \geq 0$ such that for all $i=1, \ldots, \ell$, one has: $f\left(u_{i}\right) \in F_{m+d_{i}} M$. It follows that there exists an integer $m$ such that for all $n \in \mathbb{Z}$ one has: $f\left(F_{n} M\right) \subset$ $F_{n+m} M$. Thus, we may define an increasing $\mathbb{Z}$-filtration $\mathrm{F}_{\bullet}$ End on $\operatorname{End}_{\mathbf{e}} \mathrm{H}_{\kappa} \mathbf{e}\left(\mathrm{H}_{\kappa} \mathbf{e}\right)$ by setting: $\mathrm{F}_{m}$ End $=\left\{f \in \operatorname{End}_{\mathrm{eH}_{\kappa} \mathbf{e}}\left(\mathrm{H}_{\kappa} \mathbf{e}\right) \mid f\left(F_{n} M\right) \subset F_{n+m} M, \forall n \in \mathbb{Z}\right\}$. This makes $\operatorname{End}_{\mathbf{e H}_{\kappa}} \mathbf{e}\left(\mathrm{H}_{\kappa} \mathbf{e}\right)$ a filtered ring. Observe further that $\operatorname{dim}_{\mathbb{C}}\left(\mathrm{F}_{m}\right.$ End $)<\infty$, for any $m \in \mathbb{Z}$, and if $d=$ $\operatorname{Max}\left(\operatorname{deg} u_{1}, \ldots, \operatorname{deg} u_{\ell}\right)$, then we necessarily have: $\mathrm{F}_{n}$ End $=0$, for all $n<-d$.

Now, the canonical action-map $\eta: \mathrm{H}_{\kappa} \rightarrow \operatorname{End}_{\mathrm{eH}_{\kappa} \mathbf{e}}\left(\mathrm{H}_{\kappa} \mathbf{e}\right)$ is a filtration preserving map of filtered vector spaces. Thus, to show that $\eta$ is an isomorphism, it suffices to show that 
so is $\operatorname{gr}(\eta)$. To this end, we consider the composite map:

$$
S V \# \Gamma=\operatorname{gr}\left(\mathrm{H}_{\kappa}\right) \stackrel{\operatorname{gr}(\eta)}{\longrightarrow} \operatorname{gr}\left(\operatorname{End}_{\mathrm{eH}_{\kappa} \mathbf{e}}\left(\mathrm{H}_{\kappa} \mathbf{e}\right)\right) \stackrel{j}{\longrightarrow} \operatorname{End}_{\left.\operatorname{gr}_{(\mathbf{e}} \mathrm{H}_{\kappa} \mathbf{e}\right)} \operatorname{gr}\left(\mathrm{H}_{\kappa} \mathbf{e}\right)=\operatorname{End}_{(S V)^{\Gamma}}(S V)
$$

where $j$ is the canonical map, which is clearly injective. It suffices to prove that the composite map $j \circ \operatorname{gr}(\eta)$ is bijective. The injectivity is obvious, since $j \circ \operatorname{gr}(\eta)$ becomes an isomorphism after tensoring with $\mathbb{C}\left(V^{*}\right)^{\Gamma}$, the field of $\Gamma$-invariant rational functions on $V^{*}$. To prove the surjectivity, we need to show that any endomorphism of $S V$ over $(S V)^{\Gamma}$ comes from an element of $S V \# \Gamma$.

Let $a: S V \rightarrow S V$ be an $(S V)^{\Gamma}$-linear map. Then it defines a $\mathbb{C}\left(V^{*}\right)^{\Gamma}$-linear map $\mathbb{C}\left(V^{*}\right) \rightarrow \mathbb{C}\left(V^{*}\right)$. So $a=\sum a_{g} \cdot g$, where $a_{g} \in \mathbb{C}\left(V^{*}\right), g \in \Gamma$. It is clear that the functions $a_{g}$ must be regular on $V \backslash Y$, where $Y$ is the set of points with a nontrivial isotropy group in $\Gamma$ (since on $V \backslash Y$, the surjectivity is obvious). But $Y$ has codimension $\geq 2$, so by Hartogs theorem $a_{g}$ are regular, as desired.

To prove (iii), we consider the multiplication pairing: $\mathbf{e H}_{\kappa} \otimes \mathrm{H}_{\kappa} \mathbf{e} \longrightarrow \mathbf{e H}_{\kappa} \mathbf{e}$. This pairing induces a right $\mathbf{e H}_{\kappa} \mathbf{e}$-linear map $\psi: \mathbf{e H}_{\kappa} \rightarrow \mathrm{Hom}_{\mathbf{e H}_{\kappa} \mathbf{e}}\left(\mathrm{H}_{\kappa} \mathbf{e}, \mathbf{e H}_{\kappa} \mathbf{e}\right)$, given by $\psi(x)(y)=x y$. This map is injective by part (iv), since $\psi$ is the restriction of $\eta$ to $\mathbf{e H}_{\kappa}$. It is also surjective. Indeed, by part (iv), for any $a \in\left(\mathrm{H}_{\kappa} \mathbf{e}\right)^{\vee}$, there exists $x \in \mathrm{H}_{\kappa}$ such that $x y=a(y)$ for all $y \in \mathrm{H}_{\kappa} \mathbf{e}$. But then we also have exy=a(y), since $a(y) \in \mathbf{e H}_{\kappa} \mathbf{e}$. So $a=\psi(\mathbf{e} x)$. Hence, $\psi$ is an isomorphism, and the first statement is proved. The second statement of part (iii) follows similarly. The theorem is proved.

Remark. There is an alternative proof of the isomorphisms in parts (ii) and (iii) of Theorem 1.5, along the same lines as the proof of Lemmas 1.3, 1.4 in [CBH]. That proof is less elementary and involves a lot of non-commutative algebra machinery. $\diamond$

Note that the algebra $\mathrm{H}_{t, 0}$ is isomorphic to $\mathrm{A}_{t} \# \Gamma$, provided $t \neq 0$. The latter is known, see e.g. [Mo], to be a simple algebra with trivial center. It follows by semi-continuity that, for generic values $(t, c) \in \overline{\mathrm{C}}$, the algebra $\mathrm{H}_{t, c}$ also has the trivial center. We will see below that the points $(0, c)$ are very special: the algebra $\mathrm{H}_{0, c}$ has a large center, $\mathrm{Z}_{0, c}:=\mathrm{Z}\left(\mathrm{H}_{0, c}\right)$.

Further, for any $c \in \mathrm{C}$, the construction of Hayashi [Ha], cf. $\S 15$, applied to the flat family $\left\{\mathrm{H}_{t, c}\right\}_{t \in \mathbb{C}}$, gives a Poisson algebra structure on $\mathrm{Z}_{0, c}$.

Theorem 3.1 (Satake isomorphism) For any $c \in \mathrm{C}$, the map: $\mathrm{Z}_{0, c} \rightarrow \mathbf{e H}_{0, c} \mathbf{e}, z \mapsto$ $z \cdot \mathbf{e}$, is a Poisson algebra isomorphism.

Proof. It is clear that the map: $z \mapsto z \cdot$ e gives an algebra homomorphism $\zeta: Z\left(\mathrm{H}_{0, c}\right) \rightarrow$ $\mathbf{e H}_{0, c} \mathbf{e}$. Since the algebra $\mathbf{e H}_{0, c} \mathbf{e}$ is commutative, we can also construct an algebra homomorphism $\xi: \mathbf{e H}_{0, c} \mathbf{e} \rightarrow \mathrm{Z}\left(\mathrm{H}_{0, c}\right)$ as follows. For any, $a \in \mathbf{e H}_{0, c} \mathbf{e}$, right multiplication by $a$ gives, due to commutativity of $\mathbf{e H}_{0, c} \mathbf{e}$, a right $\mathbf{e H}_{0, c} \mathbf{e}$-linear endomorphism of $\mathrm{H}_{0, c} \mathbf{e}$. This endomorphism must arise from the left action of an element $\xi(a) \in \mathrm{H}_{0, c}$, since End $_{\mathbf{e H}_{0, c} \mathbf{e}}\left(\mathrm{H}_{0, c} \mathbf{e}\right)=\mathrm{H}_{0, c}$, by Theorem 1.5(ii). Moreover, the element $\xi(a)$ belongs to the center of $\mathrm{H}_{0, c}$ since the left $\xi(a)$-action on $\mathrm{H}_{0, c} \mathbf{e}$, being induced by multiplication on the right, commutes with the left action on $\mathrm{H}_{0, c} \mathbf{e}$ of any other element of $\mathrm{H}_{0, c}$. It is clear that 
the assignment: $a \mapsto \xi(a)$ gives an algebra homomorphism $\xi: \mathbf{e H}_{0, c} \mathbf{e} \rightarrow \mathrm{Z}\left(\mathrm{H}_{0, c}\right)$, and that this homomorphism is inverse to $\zeta$.

The compatibility of the map: $z \mapsto z \cdot \mathbf{e}$ with the Poisson brackets follows directly from the "deformation-construction" of the brackets on $\mathbf{e H}_{0, c} \mathbf{e}$ and on $\mathbf{Z}\left(\mathrm{H}_{0, c}\right)$ given in $\S 15$, once we know that the integer $\mathbf{m}_{0, c}$ involved in the construction of the bracket on $\mathbf{e H}_{0, c} \mathbf{e}$ equals 1, cf. Lemma 15.1. But this has been established in the course of the proof of Theorem 1.6.

Remark. The isomorphism of Theorem 3.1 is analogous to the Satake isomorphism between the center and the spherical subalgebra of the affine Hecke algebra, due to Lusztig [L]. $\diamond$

Lemma 3.2 For $t=c=0$, we have: $\mathrm{Z}\left(\mathrm{H}_{0,0}\right)=(S V)^{\Gamma}$.

Proof. It is clear that any element of $S V \# \Gamma$ that commutes with the subalgebra $S V$ belongs to $S V$. Hence, $\mathrm{Z}_{0,0} \subset S V$. Now, an element of $S V$ commutes with $\Gamma$ if and only if it is $\Gamma$-invariant.

The imbedding: $\mathrm{Z}_{0, c} \hookrightarrow \mathrm{H}_{0, c}$ induces a canonical map: $\operatorname{gr}\left(\mathrm{Z}_{0, c}\right) \rightarrow \mathrm{Z}\left(\mathrm{H}_{0,0}\right)=\mathrm{Z}_{0,0}$.

Theorem 3.3 The canonical map is an algebra isomorphism: $\operatorname{gr}\left(\mathrm{Z}_{0, c}\right) \simeq \mathrm{Z}_{0,0}=(S V)^{\Gamma}$.

Proof. Write $\varphi: z \mapsto z \cdot \mathbf{e}$ for the map of Theorem 3.1, and $\bar{\varphi}: \mathrm{Z}_{0,0} \rightarrow \mathbf{e H}_{0,0} \mathbf{e}$ for a similar map for the algebra $\mathrm{H}_{0,0}=\operatorname{gr}\left(\mathrm{H}_{0, c}\right)$. It is clear that the maps $\varphi$ and $\bar{\varphi}$ are algebra homomorphisms. Observe that the associated graded homomorphism: $\operatorname{gr}(\varphi): \operatorname{gr}\left(\mathrm{Z}_{0, c}\right) \longrightarrow \operatorname{gr}\left(\mathbf{e H}_{0, c} \mathbf{e}\right)$ can be identified, in view of the PBW-theorem, with the composite map:

$$
\operatorname{gr}\left(\mathrm{Z}_{0, c}\right) \hookrightarrow \mathrm{Z}_{0,0}=S V^{\Gamma} \stackrel{\bar{\varphi}}{\longrightarrow} \mathbf{e H}_{0,0} \mathbf{e}=\mathbf{e} \cdot(S V \# \Gamma) \cdot \mathbf{e} .
$$

It is immediate that the second map, $\bar{\varphi}$, is an isomorphism. It follows that the map $\operatorname{gr}(\varphi)$ is injective. Now we use the following obvious claim:

Let $E=\cup_{i \geq 0} E_{i}$ and $E^{\prime}=\cup_{i \geq 0} E_{i}^{\prime}$ be two filtered vector spaces such that, for any $i \geq 0$, we have $\operatorname{dim} E_{i}=\operatorname{dim} E_{i}^{\prime}<\infty$. Assume $\varphi: E \rightarrow E^{\prime}$ is a filtration preserving linear map such that $\operatorname{gr}(\varphi)$ is injective. Then both $\operatorname{gr}(\varphi)$ and $\varphi$ are bijective maps.

Applying this observation in our situation, we conclude that the map $\operatorname{gr}(\varphi)$ gives an isomorphism $\operatorname{gr}\left(Z_{0, c}\right) \stackrel{\sim}{\longrightarrow} Z_{0,0}$, since $\operatorname{gr}\left(Z_{0, c}\right) \subset Z_{0,0}$ and the two sides have equal Poincaré series (by Theorem 3.1).

The argument in the proof Theorem 3.3 above yields the following strengthening of Theorem 3.1

Proposition 3.4 The associated graded map: $\operatorname{gr}(\varphi): \operatorname{gr}\left(\mathrm{Z}_{0, c}\right) \rightarrow \operatorname{gr}\left(\mathbf{e H}_{0, c} \mathbf{e}\right)$ is an algebra isomorphism. 
Proof of theorem 1.7: Recall that $U$ is a smooth Zariski open dense subset in Spec $Z_{0, c}$, and $\mathcal{R}_{U}$ denotes the coherent sheaf on $U$ corresponding to $\mathrm{H}_{U} \mathbf{e}$, viewed as a (finitely generated) $\mathrm{Z}_{U}$-module, so that $\Gamma\left(U, \mathcal{R}_{U}\right)=\mathrm{H}_{U}$ e. The left $\mathrm{H}_{U}$-action on $\mathrm{H}_{U}$ e gives an $\mathrm{H}_{U}$-module structure on each geometric fiber of $\mathcal{R}_{U}$.

Proving that the sheaf $\mathcal{R}_{U}$ is locally free amounts to showing that $\mathrm{H}_{U} \mathbf{e}$ is a finite rank projective $\mathrm{Z}_{U}$-module. To this end, recall the following standard result of commutative algebra. Let $A$ be a finitely generated commutative $\mathbb{C}$-algebra with unit, such that $\operatorname{Spec} A$ is a smooth algebraic variety. Then, a finitely-generated $A$-module $M$ is Cohen-Macaulay if and only if it is projective, see [Se, Ch.4, Cor.2].

Since $U$ is smooth by our assumptions, it follows from Theorem 1.5(ii) and Satake isomorphism that $\mathrm{H}_{U} \mathbf{e}$ is a finite rank projective $\mathrm{Z}_{U}$-module. Part (i) now follows from Theorem 1.5(vi).

Further, the endomorphism algebra of an algebraic vector bundle on an affine algebraic variety is always Morita equivalent to the coordinate ring on this variety. Explicitly, in our case, the equivalence is provided by the following mutually inverse functors

$$
\mathrm{H}_{U}-\bmod \underset{F}{\stackrel{G}{\rightleftarrows}} \mathrm{Z}_{U}-\bmod \quad, \quad F(M)=\mathrm{H}_{U} \mathbf{e} \otimes_{\mathrm{z}_{U}} M \quad, \quad G(N)=\mathbf{e H}_{U} \otimes_{\mathrm{H}_{U}} N=\mathbf{e} \cdot N .
$$

To prove (vi), notice that since $\mathcal{R}_{U}$ is a vector bundle it is sufficient, due to rigidity of $\Gamma$-modules, to establish that the generic fiber of $\mathcal{R}_{U}$ is isomorphic to the regular representation of $\Gamma$. But this follows from Lemma 2.24.

Remarks. (i) We have established a special case of the following well-known general result: If $A$ is an associative algebra and $\mathbf{e} \in A$ an idempotent, then $A$ is Morita equivalent to $\mathbf{e} A \mathbf{e}$ if and only if we have: $A \mathbf{e} A=A$.

(ii) We have shown that each geometric fiber of $\mathcal{R}$ over the regular locus of $\operatorname{Spec} Z_{0, c}$ is isomorphic, as a $\mathbb{C} \Gamma$-module, to the regular representation of $\Gamma$.

Proof of Proposition 1.10. Given an associative algebra $A$, one has, see [Lo], a Chern character map ch: $K(A) \rightarrow H C_{\mathrm{ev}}(A)$, where $K(A)$ stands for the Grothendieck group of finitely generated projective $A$-modules, and $H C_{\mathrm{ev}}(A)$ denotes the even degree part of the periodic cyclic homology groups of $A$, see [Lo]. The Chern character map is compatible with Morita equivalences. It is also known, see [Lo], that if $A=\mathbb{C}[X]$ is the coordinate ring of a smooth affine algebraic variety, then: $H C_{\mathrm{ev}}(A) \simeq \bigoplus_{i} H^{2 i}(X)$, the direct sum of even cohomology of $X$, as a topological space. Moreover, the map ch reduces in this case to the classical Chern character map: $K(X) \rightarrow \bigoplus_{i} H^{2 i}(X)$.

Recall now that the algebra $\mathrm{H}_{0, c}$ has a canonical increasing filtration, $F_{\bullet}\left(\mathrm{H}_{0, c}\right)$, such that $F_{0}\left(\mathrm{H}_{0, c}\right)=\mathbb{C} \Gamma$. Since $\mathrm{H}_{0, c}$ is a projective $\mathbb{C} \Gamma$-module, and the algebra $\mathrm{gr}_{\bullet} F \mathrm{H}_{0, c}$ has finite homological dimension, a general result of Quillen [Q] says that the functor: $V \mapsto \mathrm{H}_{0, c} \otimes_{\mathrm{C \Gamma}} V$ induces an isomorphism of $K$-groups: $K(\Gamma) \stackrel{\sim}{\longrightarrow} K\left(\mathrm{H}_{0, c}\right)$. Similarly, a result of Block [Bl] says that the imbedding: $\mathbb{C} \Gamma \hookrightarrow \mathrm{H}_{0, c}$ induces an isomorphism: 
$H C_{\mathrm{ev}}(\Gamma) \stackrel{\sim}{\longrightarrow} H C_{\mathrm{ev}}\left(\mathrm{H}_{0, c}\right)$. Thus, we obtain the following commutative diagram:

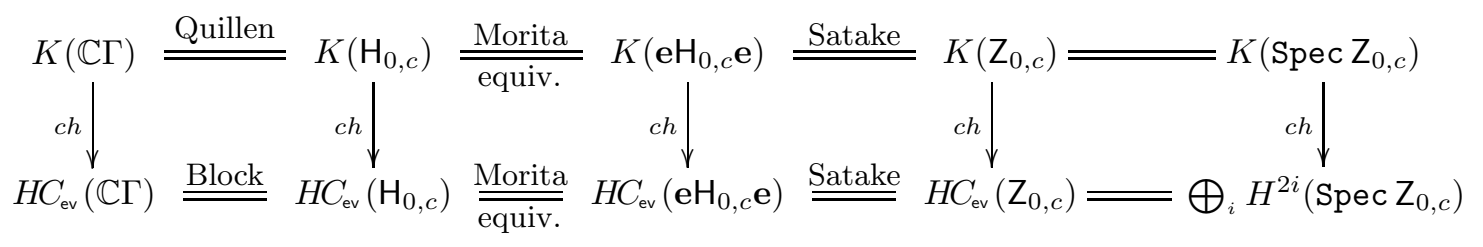

where Morita equivalence is guaranteed by Theorem 1.7(ii).

To complete the proof, it suffices to show that the leftmost vertical map in the diagram induces an isomorphism $c h_{\Gamma}: \mathbb{C} \otimes_{\mathbb{Z}} K(\mathbb{C} \Gamma) \longrightarrow H C_{\mathrm{ev}}(\mathbb{C} \Gamma)$. But the algebra $\mathbb{C} \Gamma$ is Morita equivalent to a direct sum of several copies of the field $\mathbb{C}$. Hence one may replace $\mathbb{C} \Gamma$ in the above map $c h_{\Gamma}$ by the algebra $\mathbb{C}$. The Chern character map: $\mathbb{C} \otimes_{\mathbb{Z}} K(\mathbb{C}) \longrightarrow H C_{\text {ev }}(\mathbb{C})$ is clearly an isomorphism, and we are done.

Lemma 3.5 (i) For any $c \in \mathrm{C}$, the variety $\operatorname{Spec} \mathrm{Z}_{0, c}$ is normal.

(ii) If the variety $\operatorname{Spec} Z_{0, c}$ is smooth, then the Poisson structure on $Z_{0, c}$ is nondegenerate, i.e., $\operatorname{Spec} \mathrm{Z}_{0, c}$ is a symplectic manifold.

Proof. Note that, for $c=0$, the singular locus of $\operatorname{Spec} \mathrm{Z}_{0,0}=V / \Gamma$ has codimension $\geq 2$ in $\operatorname{Spec} Z_{0,0}$. The codimension of the singular locus being semi-continuous under going to the asymptotic cone it follows that, for any $c \in \mathbf{C}$, the singular locus of $\operatorname{Spec} Z_{0, c}$ has codimension $\geq 2$ in $\operatorname{Spec} Z_{0, c}$. Further, we know that $\operatorname{Spec} Z_{0, c}$ is a Cohen-Macaulay variety, by Theorem 1.5(i). But any Cohen-Macaulay variety which is non-singular in codimension one is normal, see e.g. proof in [CG, §2.2]. Part (i) follows.

To prove (ii), assume Spec $Z_{0, c}$ is smooth, write $\beta_{c}$ for the bivector on Spec $Z_{0, c}$ giving the Poisson structure, and set $2 d=\operatorname{dim} V=\operatorname{dim}\left(\operatorname{Spec} Z_{0, c}\right)$. The set of points where the Poisson structure degenerates equals the set of points where the $2 d$-vector $\wedge^{d} \beta_{c}$ vanishes. This set is clearly a divisor (a codimension one closed subvariety) in $\operatorname{Spec} \mathrm{Z}_{0, c}$. Let $J \subset$ $\mathrm{Z}_{0, c}$ denote the defining ideal of that subvariety, and $\operatorname{gr}(J) \subset \operatorname{gr}\left(\mathrm{Z}_{0, c}\right)=\mathbb{C}[V]^{\Gamma}$, the corresponding associated graded ideal with respect to the canonical filtration on $\mathrm{H}_{0, c}$. Then $\operatorname{gr}(J)$ is a proper non-zero ideal in $\mathbb{C}[V]^{\Gamma}$, and the zero-variety of $\operatorname{gr}(J)$ is a codimension one subvariety in $V / \Gamma$, the 'asymptotic cone' to the zero-set of $\wedge^{d} \beta_{c}$. It is clear that $\wedge^{d} \beta_{0}$ is the leading homogeneous component of $\wedge^{d} \beta_{c}$, hence, vanishes on this asymptotic cone. Hence, the Poisson structure on $V / \Gamma$ must degenerate at a divisor. On the other hand, the Poisson structure on $V / \Gamma$ is non-degenerate at any smooth point, and the singular locus of $V / \Gamma$ has codimension $\geq 2$ in $V / \Gamma$. The contradiction completes the proof.

For any $c \in \mathrm{C}$, let $\operatorname{Rep}_{\mathbb{C \Gamma}}\left(\mathrm{H}_{0, c}\right)$ be the variety of all algebra homomorphisms: $\mathrm{H}_{0, c} \longrightarrow$ End $_{\mathbb{C}}(\mathbb{C} \Gamma)$, whose restriction to $\mathbb{C} \Gamma$ is the $\mathbb{C} \Gamma$-action by left multiplication. This is an affine algebraic variety. Given an $\mathrm{H}_{0, c}$-module $M \in \operatorname{Rep}_{\mathbb{C \Gamma}}\left(\mathrm{H}_{0, c}\right)$, the space $\mathbf{e} M$ is clearly a line which is stable under the action of $Z_{0, c}$. The action on this line gives an algebra homomorphism $\chi_{M}: Z_{0, c} \rightarrow \mathbb{C}$. It is clear that the assignment: $M \mapsto \chi_{M}$ gives a morphism of algebraic varieties,

$$
\pi: \operatorname{Rep}_{\mathbb{C \Gamma}}\left(\mathrm{H}_{0, c}\right) \longrightarrow \operatorname{Spec} \mathrm{Z}_{0, c}
$$


Let $\operatorname{Aut}_{\Gamma}(\mathbb{C} \Gamma)$ be the group of $\mathbb{C}$-linear automorphisms of the vector space $\mathbb{C} \Gamma$ that commute with left multiplication by $\Gamma$. The group $\operatorname{Aut}_{\Gamma}(\mathbb{C} \Gamma)$ is reductive, and it acts naturally on $\operatorname{Rep}_{\mathbb{C \Gamma}}\left(\mathrm{H}_{0, c}\right)$, preserving the fibers of the map (3.6).

Theorem 3.7 (i) There is a unique irreducible component, $\operatorname{Rep}_{\mathbb{C} \Gamma}^{\circ}\left(\mathrm{H}_{0, c}\right)$, of the variety $\operatorname{Rep}_{\mathrm{C \Gamma}}\left(\mathrm{H}_{0, c}\right)$ whose image under (3.6) is dense in $\mathrm{Spec} \mathrm{Z}_{0, c}$. The generic point of $\operatorname{Rep}_{\mathbb{C} \Gamma}^{\circ}\left(\mathrm{H}_{0, c}\right)$ is a simple $\mathrm{H}_{0, c}$-module.

(ii) The map (3.0) induces an algebra isomorphism: $\mathrm{Z}_{0, c} \stackrel{\sim}{\longrightarrow} \mathbb{C}\left[\operatorname{Rep}_{\mathbb{C} \Gamma}^{\circ}\left(\mathrm{H}_{0, c}\right)\right]^{\operatorname{Aut}_{\Gamma}(\mathbb{C} \Gamma)}$.

Proof. It follows from theorem 1.7 that any point of $\operatorname{Rep}_{\mathbb{C \Gamma}}\left(\mathrm{H}_{0, c}\right)$ containing, as a subquotient, a simple $\mathrm{H}_{0, c}$-module on which $\mathrm{Z}_{0, c}$ acts by a character that belongs to the smooth locus of Spec $Z_{0, c}$ is itself simple and, moreover, such points form a Zariski open subset in $\operatorname{Rep}_{\mathbb{C} \Gamma}\left(H_{0, c}\right)$. This set is irreducible, since so is $\operatorname{Spec} Z_{0, c}$, hence its closure, $\operatorname{Rep}_{\mathbb{C} \Gamma}^{\circ}\left(H_{0, c}\right)$, is an irreducible component of $\operatorname{Rep}_{\mathbb{C} \Gamma}\left(H_{0, c}\right)$. This proves (i).

To prove (ii), notice that the isotropy group in $\operatorname{Aut}_{\Gamma}(\mathbb{C} \Gamma)$ of a simple $\mathrm{H}_{0, c}$-module is reductive. Hence the corresponding $\operatorname{Aut}_{\Gamma}(\mathbb{C} \Gamma)$-orbit in $\operatorname{Rep}_{\mathbb{C} \Gamma}\left(\mathrm{H}_{0, c}\right)$ is closed. It follows that generic $\operatorname{Aut}_{\Gamma}(\mathbb{C} \Gamma)$-orbits in $\operatorname{Rep}_{\mathbb{C} \Gamma}^{\circ}\left(H_{0, c}\right)$ are closed, and the induced map $\pi$ :

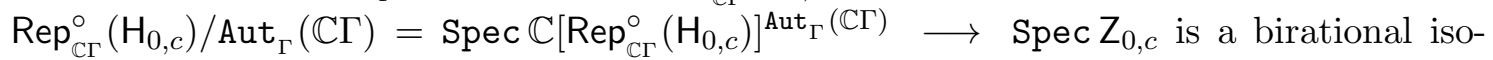
morphism. Thus, any element $f \in \mathbb{C}\left[\operatorname{Rep}_{\mathbb{C} \Gamma}^{\circ}\left(H_{0, c}\right)\right]^{\operatorname{Aut}_{\Gamma}(\mathbb{C} \Gamma)}$ descends to a rational function $\psi$ on $\operatorname{Spec} Z_{0, c}$. Moreover, Theorem 1.7 implies that $\psi$ is regular at any smooth point of Spec $Z_{0, c}$ (and the complement of smooth points has codimension $\geq 2$ ). Thus, $\psi$ extends to a regular function on the whole of $\operatorname{Spec} Z_{0, c}$, due to normality of the latter, see Lemma 3.5(i). Hence, $\psi$ is represented by an element of $Z_{0, c}$, i.e. there is $z \in Z_{0, c}$ such that the functions $f$ and $\pi^{*}(z)$ coincide on an open dense subset of $\operatorname{Rep}_{\mathbb{C} \Gamma}^{\circ}\left(\mathrm{H}_{0, c}\right)$. Thus, $f=\pi^{*}(z)$ on $\operatorname{Rep}_{\mathbb{C} \Gamma}^{\circ}\left(\mathrm{H}_{0, c}\right)$, and we are done.

Given an associative algebra $\mathcal{A}$, we write $[\mathcal{A}, \mathcal{A}]$ for the $\mathbb{C}$-vector subspace in $\mathcal{A}$ spanned by the commutators: $[a, b]_{a, b \in \mathcal{A}}$. Here is a useful criterion for the smoothness of $\operatorname{Spec} \mathrm{Z}_{0, c}$.

Proposition 3.8 For any $c \in \mathrm{C}$, and a Zariski open affine subset $U \subset$ Spec $\mathrm{Z}_{0, c}$, the following conditions are equivalent:

(i) The dimension of any simple $\mathrm{H}_{U}$-module is $\geq|\Gamma|$.

(ii) $U$ is a smooth algebraic variety.

(iii) For any $g \in \Gamma, g \neq 1$, we have: $g \in\left[\mathrm{H}_{U}, \mathrm{H}_{U}\right]$.

(iv) For any simple $\mathrm{H}_{U}$-module $M$, and any $g \in \Gamma, g \neq 1$, we have: $\operatorname{Tr}(g, M)=0$.

In particular, if any of the conditions (i), (iii) or (vi) holds for $U=\operatorname{Spec} Z_{0, c}$, then $\operatorname{Spec} Z_{0, c}$ is smooth.

We refer the reader to $\S 16$ for some applications of Proposition 3.8 .

Remark. It will be clear from the proof of the Proposition that conditions (i)-(vi) imply automatically that the restriction to $\Gamma$ of any simple $\mathrm{H}_{U}$-module is isomorphic to the regular representation of $\Gamma$. $\diamond$ 
Our proof of the Proposition will be based on the following important construction that will also play a role later, in $\S 11$.

Let $\widehat{\mathcal{R}}: \mathbb{C} \Gamma \times \operatorname{Rep}_{\mathbb{C} \Gamma}^{\circ}\left(\mathrm{H}_{0, c}\right) \longrightarrow \operatorname{Rep}_{\mathbb{C} \Gamma}^{\circ}\left(\mathrm{H}_{0, c}\right)$ be the trivial vector bundle on the variety $\operatorname{Rep}_{\mathbb{C} \Gamma}^{\circ}\left(\mathrm{H}_{0, c}\right)$, viewed as an $\operatorname{Aut}_{\Gamma}(\mathbb{C} \Gamma)$-equivariant $\mathcal{O}$-sheaf with geometric fiber $\mathbb{C} \Gamma$ (so that the group $\operatorname{Aut}_{\Gamma}(\mathbb{C} \Gamma)$ acts simultaneously on both $\operatorname{Rep}_{\mathbb{C} \Gamma}^{\circ}\left(H_{0, c}\right)$ and $\left.\mathbb{C} \Gamma\right)$. There is a canonical $\mathrm{H}_{0, c}$-module structure on $\widehat{\mathcal{R}}$ defined as follows. A point $\xi \in \operatorname{Rep}_{\mathbb{C} \Gamma}^{\circ}\left(\mathrm{H}_{0, c}\right)$ is by definition an algebra homomorphism $\xi: \mathrm{H}_{0, c} \rightarrow \operatorname{End}_{\mathbb{C}}(\mathbb{C} \Gamma)$. We let $\mathrm{H}_{0, c}$ act on $\widehat{\mathcal{R}}_{\xi} \simeq \mathbb{C} \Gamma$, the fiber of $\widehat{\mathcal{R}}$ at $\xi$, via the homomorphism $\xi$. The $\mathrm{H}_{0, c}$-module structure on $\widehat{\mathcal{R}}$ thus defined clearly commutes with the $\operatorname{Aut}_{\Gamma}(\mathbb{C} \Gamma)$-action.

Let $\operatorname{PAut}_{\Gamma}(\mathbb{C} \Gamma)=\operatorname{Aut}_{\Gamma}(\mathbb{C} \Gamma) / \mathbb{C}^{*}$ be the quotient modulo the scalars. We have the natural projection: $\operatorname{Aut}_{\Gamma}(\mathbb{C} \Gamma) \rightarrow \operatorname{PAut}_{\Gamma}(\mathbb{C} \Gamma)$, and the $\operatorname{Aut}_{\Gamma}(\mathbb{C} \Gamma)$-action on $\operatorname{Rep}_{\mathbb{C} \Gamma}\left(\mathrm{H}_{0, c}\right)$ (but not on $\mathbb{C} \Gamma$ ) factors throught the group PAut $_{\Gamma}(\mathbb{C} \Gamma)$. We define a canonical splitting: $\operatorname{PAut}_{\Gamma}(\mathbb{C} \Gamma) \hookrightarrow \operatorname{Aut}_{\Gamma}(\mathbb{C} \Gamma)$ of the natural projection by the requirement, that the image of $\operatorname{PAut}_{\Gamma}(\mathbb{C} \Gamma)$ in $\operatorname{Aut}_{\Gamma}(\mathbb{C} \Gamma)$ acts trivially on the element $\mathbf{e} \in \mathbb{C} \Gamma$. This splitting makes it possible to view the bundle: $\mathbb{C} \Gamma \times \operatorname{Rep}_{\mathbb{C} \Gamma}^{\circ}\left(\mathrm{H}_{0, c}\right) \rightarrow \operatorname{Rep}_{\mathbb{C} \Gamma}^{\circ}\left(\mathrm{H}_{0, c}\right)$ as a PAut $\operatorname{Pa}_{\Gamma}(\mathbb{C} \Gamma)$-equivariant $\mathrm{H}_{0, c^{-}}$-vector bundle.

Proof of Proposition 3.8: (i) $\Longrightarrow$ (ii) If all simple $\mathrm{H}_{U}$-modules have dimension $\geq|\Gamma|$ then each point $\xi \in \operatorname{Rep}_{\mathbb{C} \Gamma}^{\circ}\left(\mathrm{H}_{U}\right)$ must represent a simple $\mathrm{H}_{U}$-module. Hence, the isotropy group of $\xi$ in $\operatorname{Aut}_{\Gamma}(\mathbb{C} \Gamma)$ reduces to scalars $\mathbb{C}^{*} \subset \operatorname{Aut}_{\Gamma}(\mathbb{C} \Gamma)$, by Schur lemma. Therefore, $\operatorname{PAut}_{\Gamma}(\mathbb{C} \Gamma)$, a reductive group, acts freely on $\operatorname{Rep}_{\mathbb{C} \Gamma}^{\circ}\left(\mathrm{H}_{U}\right)$. Hence, all PAut $(\mathbb{C} \Gamma)$-orbits in $\operatorname{Rep}_{\mathbb{C} \Gamma}^{\circ}\left(\mathrm{H}_{U}\right)$ are closed, and are exactly the fibers of the projection $\pi_{U}: \operatorname{Spec}^{\operatorname{Rep}_{\mathbb{C} \Gamma}^{\circ}}\left(\mathrm{H}_{U}\right) \rightarrow$

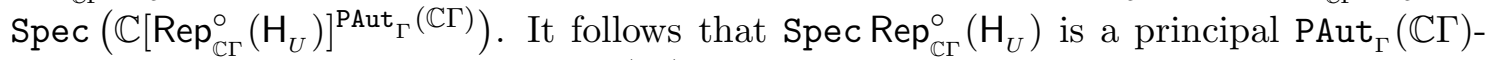
bundle over Spec $\left(\mathbb{C}\left[\operatorname{Rep}_{\mathbb{C} \Gamma}^{\circ}\left(\mathrm{H}_{U}\right)\right]^{\text {PAut }_{\Gamma}(\mathbb{C} \Gamma)}\right.$ ) (in étale topology). Therefore, the $\operatorname{PAut}_{\Gamma}(\mathbb{C} \Gamma)$ equivariant vector bundle $\widehat{\mathcal{R}}_{U}$ on $\operatorname{Rep}_{\mathbb{C} \Gamma}^{\circ}\left(\mathrm{H}_{U}\right)$ descends to a well-defined algebraic vector bundle $\widetilde{\mathcal{R}}_{U}$ on $\operatorname{Spec}\left(\mathbb{C}\left[\operatorname{Rep}_{\mathbb{C} \Gamma}^{\circ}\left(\mathrm{H}_{U}\right)\right]^{\operatorname{Aut}_{\Gamma}(\mathbb{C} \Gamma)}\right)$. Moreover, the $\mathrm{H}_{U}$-module structure on $\widehat{\mathcal{R}}_{U}$ descends to an $\mathrm{H}_{U}$-module structure on $\widetilde{\mathcal{R}}_{U}$.

Further, by Theorem 3.7(ii), we may (and will) identify $\operatorname{Spec}\left(\mathbb{C}\left[\operatorname{Rep}_{\mathbb{C} \Gamma}^{\circ}\left(\mathrm{H}_{U}\right)\right]^{\operatorname{Aut}_{\Gamma}(\mathbb{C} \Gamma)}\right)$ with $U$, hence view $\widetilde{\mathcal{R}}_{U}$ as a locally free coherent sheaf on $U$. The (constant) section of $\widehat{\mathcal{R}}_{U}$ corresponding to the element $\mathbf{e} \in \mathbb{C} \Gamma$ gives rise to a non-vanishing regular section of $\widetilde{\mathcal{R}}_{U}$, to be denoted $\widetilde{\mathbf{e}}$. Using the $\mathrm{H}_{U}$-action on $\widetilde{\mathcal{R}}_{U}$, we define an $\mathrm{H}_{U}$-module map: $\mathrm{H}_{U} \mathbf{e} \longrightarrow \Gamma(U, \widetilde{\mathcal{R}})$ by the assignment: $u \cdot \mathbf{e} \mapsto u \cdot \widetilde{\mathbf{e}}$. This gives an $\mathrm{H}_{U}$-equivariant morphism $\epsilon: \mathcal{R}_{U} \rightarrow \widetilde{\mathcal{R}}_{U}$ of coherent sheaves on $U$. The morphism $\epsilon$ is clearly an isomorphism over the generic point of $U$, hence is injective since the sheaf $\mathcal{R}_{U}$, being reflexive, is torsion free. On the other hand, for any $\chi \in U$, the induced map on the fibers: $\mathcal{R}_{\chi} \rightarrow \widetilde{\mathcal{R}}_{\chi}$ is a non-zero map of $\mathrm{H}_{U}$-modules, hence is surjective, since $\widetilde{\mathcal{R}}_{\chi}$ is a simple $\mathrm{H}_{U}$-module by our hypothesis (i). Therefore, $\epsilon$ is an isomorphism, by Nakayama lemma. Thus, the sheaf $\mathcal{R}_{U}$ is also locally free.

Recall that, according to a well known criterion, a finitely generated commutative algebra $\mathcal{A}$ is smooth (i.e. the scheme $\operatorname{Spec} \mathcal{A}$ is smooth) if and only if $\mathcal{A}$ has finite (global) homological dimension. Thus, $U$ is smooth if and only if $Z_{U}$ has finite (global) homological dimension. On the other hand, since $S V$ is smooth, the algebra $\operatorname{gr}\left(\mathrm{H}_{0, c}\right) \simeq S V \# \Gamma$, clearly 
has finite homological dimension. It follows that both the algebra $\mathrm{H}_{0, c}$ and $\mathrm{H}_{U}$ have finite homological dimension. Further, by Theorem 1.7 we have: $\mathrm{H}_{U} \simeq \operatorname{End}_{\mathrm{z}_{U}} \mathcal{R}_{U}$. It follows that, if $\mathcal{R}_{U}$ is a vector bundle, then the algebra $\mathrm{H}_{U}$ is Morita equivalent to $\mathrm{Z}_{U}$. Hence the algebras $\mathrm{Z}_{U}$ and $\mathrm{H}_{U}$ have equal global homological dimensions, and (ii) follows.

(ii) $\Longrightarrow$ (iii) Set $Z=Z_{U}$. We know that $\mathrm{H}_{U} \simeq \operatorname{End}_{\mathrm{Z}} \mathcal{R}_{U}$, by Theorem 1.7. Note that since any element of $\left[\operatorname{End}_{\mathrm{z}} \mathcal{R}_{U}, \operatorname{End}_{\mathrm{z}} \mathcal{R}_{U}\right]$ acts by a trace zero operator in each fiber of $\mathcal{R}_{U}$, we have: $\mathrm{Z} \cap\left[\operatorname{End}_{\mathrm{z}} \mathcal{R}_{U}, \operatorname{End}_{\mathrm{z}} \mathcal{R}_{U}\right]=0$. Further, the algebra $\operatorname{End}_{\mathrm{z}} \mathcal{R}_{U}$ being Morita equivalent to Z, the imbedding: $\mathrm{Z} \hookrightarrow \mathrm{H}_{U}=$ End $_{\mathrm{Z}} \mathcal{R}_{U}$ induces a bijection: $\mathrm{Z}=\mathrm{Z} /[\mathrm{Z}, \mathrm{Z}] \stackrel{\sim}{\longrightarrow} \mathrm{H}_{U} /\left[\mathrm{H}_{U}, \mathrm{H}_{U}\right]$. It follows that there is a direct sum decomposition:

$$
\mathrm{H}_{U}=\mathrm{Z}_{U} \bigoplus\left[\mathrm{H}_{U}, \mathrm{H}_{U}\right]
$$

Now fix $g \in \Gamma, g \neq 1$. Using the decomposition above we can write: $g=z+u$, where $z \in \mathrm{Z}_{U}$, and $u \in\left[\mathrm{H}_{U}, \mathrm{H}_{U}\right]$. Since the trace of $g$ in the regular representation vanishes, the element $g=z+u$ acts by a trace zero operator in each fiber of $\mathcal{R}_{U}$, by Theorem 1.7(vi). It follows that $z=0$. Thus, $g=u \in\left[\mathrm{H}_{U}, \mathrm{H}_{U}\right]$.

The implication (iii) $\Longrightarrow$ (iv) is clear.

(iv) $\Longrightarrow$ (i)

Assume that for any $g \in \Gamma, g \neq 1$, the action of $g$ in any simple (in particular, finite-dimensional) $\mathrm{H}_{U}$-module $M$ has vanishing trace: $\operatorname{Tr}(g, M)=0$. It follows from representation theory of finite groups that the restriction of any simple $\mathrm{H}_{U}$-module $M$ to $\Gamma$ is isomorphic to a multiple of the regular representation of $\Gamma$.

Remark. There is an alternative proof of the Morita equivalence in the implication (i) $\Longrightarrow$ (ii) of the Proposition above, based on a general result of M. Artin (1968) which in the special situation we need reads:

Let $A$ be a $\mathbb{C}$-algebra with center Z, finitely generated as a Z-module, and such that:

- Z has no zero divisors;

- A injects into $A \otimes_{\mathrm{Z}} Q(\mathrm{Z})$, where $Q(\mathrm{Z})$ is the field of fractions of Z;

- Any irreducible A-module has dimension $N$;

- $A \otimes_{\mathrm{Z}} Q(\mathrm{Z})=\operatorname{Mat}_{N}(Q(\mathrm{Z}))$.

Then $A$ is Morita equivalent to $\mathbf{Z}$.

The proof of this result makes a nontrivial use of the theory of polynomial identities. $\diamond$

Proof of part (ii) of theorem 1.8: The first claim of part (ii) is just Lemma 3.5(i).

To proceed further, fix $c \in C$ such that $\operatorname{Spec}\left(\mathbf{e H}_{0, c} \mathbf{e}\right)$ is smooth. The family of noncommutative associative algebras $\left\{\mathrm{H}_{t, c}\right\}_{t \in \mathbb{C}}$ may be viewed as a single $\mathbb{C}[t]$-algebra, which is a polynomial deformation of the algebra $\mathrm{H}_{0, c}$. Let $\hat{\mathrm{H}}_{t, c}$ denote the $t$-adic completion of that $\mathbb{C}[t]$-algebra, a formal flat deformation of $\mathrm{H}_{0, c}$. Thus, $\mathbf{e} \hat{\mathrm{H}}_{t, c} \mathbf{e}$ is a formal flat deformation of $\mathbf{e H}_{0, c} \mathbf{e}$. Recall that this deformation gives a Poisson algebra structure on $\mathbf{e H}_{0, c} \mathbf{e}$. Since $\operatorname{Spec}\left(\mathbf{e H}_{0, c} \mathbf{e}\right)$ is smooth, hence a symplectic manifold, we may apply the results of Appendix D to that deformation. 
Write $\mathcal{K}=\mathbb{C}((t))$ for the field of formal Laurent series, and let $\mathrm{H}_{\mathcal{K}}:=\mathcal{K} \widehat{\otimes}_{\mathbb{C}[t]]} \hat{\mathrm{H}}_{t, c}$ be the $\mathcal{K}$-algebra obtained from $\hat{\mathrm{H}}_{t, c}$ by localizing at $t$. Thus, Lemma 15.7 and Proposition 15.2 applied to the algebra $\mathcal{A}=\mathbf{e} \hat{\mathrm{H}}_{t, c} \mathbf{e}$ yield a graded $\mathcal{K}$-algebra isomorphism:

$$
H H^{\bullet}\left(\mathbf{e H}_{\mathcal{K}} \mathbf{e}\right) \simeq \mathcal{K} \otimes_{\mathbb{C}} H^{\bullet}\left(\operatorname{Spec}\left(\mathbf{e H}_{0, c} \mathbf{e}\right)\right) .
$$

Theorem 1.7 and Proposition 15.8 imply that the $\mathcal{K}$-algebra $\mathbf{e H}_{\mathcal{K}} \mathbf{e}$ is Morita equivalent to $\mathrm{H}_{\mathcal{K}}$. Hence these two algebras have canonically isomorphic Hochschild cohomology. Thus, part (i) of Theorem 1.8 and isomorphism (3.9) yield the following chain of $\mathcal{K}$-algebra isomorphisms, that completes the proof of part (ii) of Theorem 1.8

$$
\mathcal{K} \otimes_{\mathbb{C}} \operatorname{gr}_{\bullet}^{F} \mathrm{Z \Gamma} \stackrel{\sim}{\longrightarrow} H H^{\bullet}\left(\mathrm{H}_{\mathcal{K}}\right) \simeq H H^{\bullet}\left(\mathbf{e H}_{\mathcal{K}} \mathbf{e}\right) \simeq \mathcal{K} \otimes_{\mathbb{C}} H^{\bullet}\left(\operatorname{Spec}\left(\mathbf{e H}_{0, c} \mathbf{e}\right)\right)
$$

Let $\Gamma \subset S p(V)$ be any finite group, and $\Gamma^{\circ} \subset \Gamma$ the subgroup generated by all symplectic reflections in $\Gamma$. Write $\mathrm{H}_{t, c}^{\circ}$ for the symplectic reflection algebra constructed out of $V$ and $\Gamma^{\circ}$ (instead of $\Gamma$ ), i.e., $\mathrm{H}_{t, c}^{\circ} \subset \mathrm{H}_{t, c}$ is the subalgebra generated by $V$ and $\Gamma^{\circ}$. Then $\Gamma^{\circ}$ is a normal subgroup of $\Gamma$, and the algebra $\mathrm{H}_{t, c}$ is an 'extension' of $\mathrm{H}_{t, c}^{\circ}$ by $\Gamma / \Gamma^{\circ}$. Specifically, we have: $\mathrm{H}_{t, c} \simeq \mathrm{H}_{t, c}^{\circ} \#_{\Gamma \circ} \Gamma$. Here, for any normal subgroup $H \triangleleft \Gamma$ and any $\Gamma$-algebra $A$ containing $\mathbb{C} H$, we write $A \#_{H} \Gamma$ for the vector space $A \bigotimes_{\mathbb{C} H} \mathbb{C} \Gamma$ equipped with the natural algebra structure. Thus, understanding general symplectic reflection algebras reduces to understanding those associated with groups $\Gamma$ generated by symplectic reflections.

Verbitsky has shown in [Ve] that a necessary condition for the existence of a smooth symplectic resolution of singularities $p: \widehat{V / \Gamma} \rightarrow V / \Gamma$ is that the group $\Gamma$ is generated by symplectic reflections. We have an analogue of that result for varieties $\operatorname{Spec} Z_{0, c}$. Specifically, given any finite group $\Gamma \subset S p(V)$, fix $c \in \mathrm{C}$ and let $\mathrm{H}_{0, c}$ be the corresponding symplectic reflection algebra. Let $\Gamma^{\circ} \subset \Gamma$ be the subgroup generated by those symplectic reflections $s \in S$ for which $c_{s} \neq 0$.

Proposition 3.10 If Spec $Z_{0, c}$ is smooth then $\Gamma^{\circ}=\Gamma$.

Proof. It is clear that $\Gamma^{\circ}$ is a normal subgroup in $\Gamma$. Set $K=\Gamma / \Gamma^{\circ}$. Let $\mathrm{H}_{0, c}^{\circ}$ be the symplectic reflection algebra corresponding to the group $\Gamma^{\circ}$ (as opposed to $\Gamma$ ). As we have mentioned above the algebra $\mathrm{H}_{0, c}$ is an extension of $\mathrm{H}_{0, c}^{\circ}$ by $K$, and in particular it is a free $\mathrm{H}_{0, c}^{\circ}$-module (both left and right) of rank $|K|$.

Let $\mathrm{Z}_{0, c}^{\circ}$ be the center of $\mathrm{H}_{0, c}^{\circ}$. Then $K$ acts on $\mathrm{Z}_{0, c}^{\circ}$ by conjugation, and $\mathrm{Z}_{0, c}=\left(\mathrm{Z}_{0, c}^{\circ}\right)^{K}$. So if $\mathcal{M}_{c}$ and $\mathcal{M}_{c}^{\circ}$ denote the spectra of these algebras then $\mathcal{M}_{c}=\mathcal{M}_{c}^{\circ} / K$.

We have assumed that $\mathcal{M}_{c}=\operatorname{Spec} \mathrm{Z}_{0, c}$ is smooth. We claim that in this case $\mathcal{M}_{c}^{\circ}$ is smooth also. Indeed, by Proposition 3.8, every finite dimensional $\mathrm{H}_{0, c}$-module is of dimension at least $|\Gamma|$. If there were a finite dimensional $\mathrm{H}_{0, c}^{\circ}$-module of dimension $<\left|\Gamma^{\circ}\right|$, one could induce from $\mathrm{H}_{0, c}^{\circ}$ to $\mathrm{H}_{0, c}$, and get a contradiction. Thus, all simple $\mathrm{H}_{0, c}^{\circ}$-modules are of dimension $\geq\left|\Gamma^{\circ}\right|$, hence by the same proposition we have $\mathcal{M}_{c}^{\circ}$ is smooth. We claim that $K$ acts freely on $\mathcal{M}_{c}^{\circ}$. Indeed, assume for some $\gamma \in K, \gamma \neq 1$, the fixed point set $\left(\mathcal{M}_{c}^{\circ}\right)^{\gamma}$ is not empty. Let $m \in\left(\mathcal{M}_{c}^{\circ}\right)^{\gamma}$ be a smooth point, and write $K^{\prime} \subset K$ for the finite group generated by $\gamma$. The group $K^{\prime}$ acts linearly on $T_{m}\left(\mathcal{M}_{c}^{\circ}\right)$, the tangent space at 
$m$. We observe that, by Chevalley theorem, the orbifold $T_{m}\left(\mathcal{M}_{c}^{\circ}\right) / K^{\prime}$ can not be smooth, since the group $K^{\prime}$ acts on $T_{m}\left(\mathcal{M}_{c}^{\circ}\right)$ by symplectic automorphisms, hence is not generated by (ordinary) reflections. It follows that $m$ projects to a singular point of $\mathcal{M}_{c}=\mathcal{M}_{c}^{\circ} / K$. But since $\mathcal{M}_{c}$ has been shown to be smooth, it follows that $K$ acts freely on $\mathcal{M}_{c}^{\circ}$.

Let $\mathrm{Z} \Gamma^{\circ}$ be the center of the group algebra $\mathbb{C} \Gamma^{\circ}$. By Theorem 1.8, the cohomology of $\mathcal{M}_{c}^{\circ}$ is isomorphic, as a graded space, to $\operatorname{gr}\left(Z \Gamma^{\circ}\right)$. Moreover, it is clear from the proof of this theorem that this isomorphism can be chosen to be $K$-equivariant.

Now let $g$ be a nontrivial element of $K$. By the Lefschetz fixed point formula for $g$, an automorphism of finite order, we have $\operatorname{Tr}\left(g, H^{*}\left(\mathcal{M}_{c}^{\circ}\right)\right)=0$. On the other hand,

$$
\operatorname{Tr}\left(g, H^{*}\left(\mathcal{M}_{c}^{\circ}\right)\right)=\operatorname{Tr}\left(g, \operatorname{gr}\left(Z \Gamma^{\circ}\right)\right)=\operatorname{Tr}\left(g, Z \Gamma^{\circ}\right),
$$

where the rightmost term equals the number of conjugacy classes in $\Gamma^{\circ}$, hence, is $>0$. The contradiction implies that $g$ does not exist. Thus, the group $K$ is trivial, and $\Gamma^{\circ}=\Gamma$.

\section{The rational Cherednik algebra}

Let $\mathfrak{h}$ be a finite dimensional complex vector space with a nondegenerate inner product, and $W$ a finite group of orthogonal transformations of $\mathfrak{h}$ generated by reflections. For any reflection $s \in W$, fix a nonzero linear function $\alpha_{s} \in \mathfrak{h}^{*}$ uniquely determined, up to a nonzero scalar factor, by the condition: $s\left(\alpha_{s}\right)=-\alpha_{s}$. We choose the scalar factors in such a way that the function: $s \mapsto \alpha_{s}^{2}$ is $W$-invariant. Write $R$ for the collection of linear functions $\left\{ \pm \alpha_{s}\right\}_{s=\text { reflection in } W}$. For $\alpha \in R \subset \mathfrak{h}^{*}$, we let $s_{\alpha}$ denote the corresponding reflection $s$, and set $\alpha^{\vee}:=2 \frac{(\alpha,-)}{(\alpha, \alpha)} \in \mathfrak{h}$ (we note that $(\alpha, \alpha) \neq 0$ since $\mathbb{C} \cdot \alpha$ and $\alpha^{\perp}$ are eigenspaces of $s$ with different eigenvalues, and therefore have zero intersection). A point $x \in \mathfrak{h}$ is called regular if, for all $\alpha \in R$, we have: $\alpha(x) \neq 0$.

From now on, we fix a complex-valued $W$-invariant function $c: R \rightarrow \mathbb{C}, \alpha \mapsto c_{\alpha}$, and $t \in \mathbb{C}$. Define a rational Cherednik algebra with parameters $(t, c)$ to be the symplectic reflection algebra corresponding to the $W$-diagonal action on $\mathfrak{h} \oplus \mathfrak{h}^{*}$. Explicitly, it is an associative $\mathbb{C}$-algebra $\mathrm{H}_{t, c}$ generated by the spaces $\mathfrak{h}, \mathfrak{h}^{*}$, and the group $W$, with defining relations (1.15).

Example: $\mathbf{S}_{\mathbf{n}}$-case. In the special case of the root system $R=\mathbf{A}_{\mathbf{n}-\mathbf{1}}$ in the vector space $\mathfrak{h}=\mathbb{C}^{n}$ (not in $\mathbb{C}^{n-1}$ ) we have: $W=S_{n}$. Use the standard coordinates on $\mathbb{C}^{n}$ to write $\mathbb{C}[\mathfrak{h}]=\mathbb{C}\left[x_{1}, \ldots, x_{n}\right]$ and $\mathbb{C}\left[\mathfrak{h}^{*}\right]=\mathbb{C}\left[y_{1}, \ldots, y_{n}\right]$. Recall that, in the $\mathbf{A}_{\mathbf{n}-\mathbf{1}_{\text {-case, }} \text {, all }}$ roots are $W$-conjugate and the function $c: R \rightarrow \mathbb{C}$ reduces to a constant; we will assume that $c=1$, and will view the parameter $\kappa=(t, 1)$ as a point of $\mathbb{P}^{1}$. Thus, the case $t=0$ corresponds to the point $\kappa=\infty$.

Write $s_{i j} \in S_{n}$ for the transposition: $i \longleftrightarrow j$. The algebra $\mathrm{H}_{t}=\mathrm{H}_{t, 1}\left(S_{n}\right)$ has generators $x_{1}, \ldots, x_{n}, y_{1}, \ldots, y_{n}$ and the group $S_{n}$, with the following defining relations, which are a 
specialization of 1.15:

$$
\begin{array}{ll}
s_{i j} \cdot x_{i}=x_{j} \cdot s_{i j} \quad, \quad s_{i j} \cdot y_{i}=y_{j} \cdot s_{i j}, & \forall i, j \in\{1,2, \ldots, n\}, i \neq j \\
{\left[y_{i}, x_{j}\right]=s_{i j} \quad, \quad\left[x_{i}, x_{j}\right]=0=\left[y_{i}, y_{j}\right],} & \forall i, j \in\{1,2, \ldots, n\}, i \neq j \\
{\left[y_{k}, x_{k}\right]=t \cdot 1-\sum_{i \neq k} s_{i k} .} &
\end{array}
$$

The relations (4.1) have an intriguing 'hidden' symmetry. Specifically, for each $i=$ $1,2, \ldots, n$, put: $z_{i}=x_{i} \cdot y_{i}-\sum_{j<i} s_{i j} \in \mathrm{H}_{t}$. Let $\mathcal{H}$ be the degenerate affine Hecke algebra of type $\mathbf{A}_{\mathbf{n}-\mathbf{1}}$. Recall that the latter algebra contains $\mathbb{C} S_{n}$ and $\mathbb{C}\left[u_{1}, \ldots, u_{n}\right]$ as subalgebras, subject to certain commutation relations, defined e.g. in [Dr2]. Given any non-zero triple $(a, b, c) \in \mathbb{C}^{3}$, we consider an assignment:

$$
\varpi_{a, b, c}: u_{i} \mapsto a x_{i}+b y_{i}+c z_{i}, i=1,2, \ldots, n, \quad \text { and } \quad w \mapsto w, \forall w \in S_{n} .
$$

The Proposition below is verified by a straightforward calculation (Alternatively, one may observe that in the faithful representation $\widetilde{\Theta}_{t, c}$, see Proposition 4.5 below, the $z_{i}$ 's go, after a change of variable, to 'trigonometric' Dunkl operators. The latter are known to commute with each other by Cherednik). We do not know if this Proposition has a generalization to root systems other than $\mathbf{A}_{\mathbf{n}-\mathbf{1}}$.

Proposition 4.3 (i) The elements: $\left\{a x_{i}+b y_{i}+c z_{i}\right\}_{i=1,2, \ldots, n}$ commute in $\mathrm{H}_{t}$, for any triple $(a, b, c) \in \mathbb{C}^{3} \backslash\{(0,0,0)\}$.

(ii) For any $c \neq 0$, the assignment (4.9) extends to an algebra imbedding $\varpi_{a, b, c}: \mathcal{H} \hookrightarrow$ $\mathrm{H}_{t}$. For $c=0$ (and any $(a, b) \neq(0,0)$ ), the map $\varpi_{a, b, c}$ degenerates to an algebra imbedding $\varpi_{a, b, 0}: \mathbb{C}\left[u_{1}, \ldots, u_{n}\right] \# S_{n} \hookrightarrow \mathrm{H}_{t}$.

We now return to Cherednik algebras associated to a general root system. Theorem 1.3 yields another useful version of the PBW-theorem for Cherednik algebras:

Corollary 4.4 For any $\kappa \in \overline{\mathrm{C}}$, multiplication in $\mathrm{H}_{\kappa}$ induces a vector space isomorphism: $\mathbb{C}[\mathfrak{h}] \otimes \mathbb{C}\left[\mathfrak{h}^{*}\right] \otimes \mathbb{C} W \stackrel{\sim}{\longrightarrow} \mathrm{H}_{\kappa}$.

Representation via Dunkl operators. Regular points form a Zariski open subset $\mathfrak{h}^{\text {reg }} \subset \mathfrak{h}$, and we write $\mathcal{D}\left(\mathfrak{h}^{\text {reg }}\right)$ for the algebra of algebraic differential operators on $\mathfrak{h}^{\text {reg }}$. Given $\kappa=(t, c) \in \mathbb{C} \oplus \mathfrak{C}$, to any $y \in \mathfrak{h}$ one associates the following Dunkl operator $([\mathrm{D} 1, \mathrm{D} 2])$ :

$$
D_{y}:=t \frac{\partial}{\partial y}+\frac{1}{2} \sum_{\alpha \in R} c_{\alpha} \cdot \frac{\langle\alpha, y\rangle}{\alpha} \cdot\left(s_{\alpha}-1\right) \in \mathcal{D}\left(\mathfrak{h}^{\mathrm{reg}}\right) \# W
$$

We recall the following well known result, due to Cherednik in the trigonometric case.

Proposition 4.5 For any $\kappa=(t, c) \in \mathbb{C} \oplus \mathbb{C}, t \neq 0$, the assignment: $w \mapsto w, x \mapsto$ $x, y \mapsto D_{y}, w \in W, x \in \mathfrak{h}^{*}, y \in \mathfrak{h}$, extends to an injective algebra homomorphism $\widetilde{\Theta}_{t, c}$ : $\mathrm{H}_{t, c} \hookrightarrow \mathcal{D}\left(\mathfrak{h}^{\mathrm{reg}}\right) \# W$. 
Proof. The construction of the homomorphism $\widetilde{\Theta}_{t, c}$ has the following conceptual interpretation. Consider the $\mathrm{H}_{t, c}$-module $\operatorname{Ind}_{S \mathfrak{h} \# W} \mathrm{H}_{t, c} \mathbf{1}$, induced from the trivial representation of the subalgebra $S \mathfrak{h} \# W \subset \mathrm{H}_{t, c}$. The underlying vector space of this module can be identified, by PBW-isomorphism 4.4, with $\mathbb{C}[\mathfrak{h}]$, the polynomial algebra. Writing explicitly the action on polynomials of the generators of the algebra $\mathrm{H}_{t, c}$ yields the formulas for $\widetilde{\Theta}_{t, c}$.

To prove injectivity of $\widetilde{\Theta}_{t, c}$, equip the algebra $\mathrm{H}_{t, c}$ with an increasing filtration $F_{\bullet}\left(\mathrm{H}_{t, c}\right)$, by placing both $\mathbb{C} W$ and $\mathfrak{h}^{*}$ in filtration degree zero, and $\mathfrak{h}$ in filtration degree 1 (this filtration is different from the canonical filtration on $\mathrm{H}_{t, c}$ introduced earlier). Clearly, for any $k \geq 0$, the homomorphism $\widetilde{\Theta}_{t, c}: \mathrm{H}_{t, c} \longrightarrow \mathcal{D}\left(\mathfrak{h}^{\text {reg }}\right) \# W$ takes elements of $F_{k}\left(\mathrm{H}_{t, c}\right)$ into $\mathbb{C} W$-valued differential operators on $\mathfrak{h}^{\text {reg }}$ of order $\leq k$. Thus, there is a well-defined associated graded map

$$
\operatorname{gr}_{F}\left(\widetilde{\Theta}_{t, c}\right): \operatorname{gr}_{F}\left(\mathrm{H}_{t, c}\right) \longrightarrow \operatorname{gr}\left(\mathcal{D}\left(\mathfrak{h}^{\mathrm{reg}}\right) \# W\right) \simeq \mathbb{C}\left[\mathfrak{h}^{\text {reg }} \times \mathfrak{h}^{*}\right] \# W .
$$

Note that the explicit formula for the Dunkl operator shows that the image of $\operatorname{gr}_{F}\left(\mathrm{H}_{t, c}\right)$ equals the subalgebra: $\mathbb{C}\left[\mathfrak{h} \times \mathfrak{h}^{*}\right] \# W \subset \mathbb{C}\left[\mathfrak{h}^{\text {reg }} \times \mathfrak{h}^{*}\right] \# W$. We consider the composition:

$$
\mathbb{C}\left[\mathfrak{h} \oplus \mathfrak{h}^{*}\right] \# W=\mathrm{H}_{0,0} \stackrel{\sim}{\longrightarrow} \operatorname{gr}\left(\mathrm{H}_{t, c}\right) \stackrel{\operatorname{gr}_{F}\left(\widetilde{\Theta}_{t, c}\right)}{\longrightarrow} \mathbb{C}\left[\mathfrak{h} \times \mathfrak{h}^{*}\right] \# W \subset \mathbb{C}\left[\mathfrak{h}^{\text {reg }} \times \mathfrak{h}^{*}\right] \# W .
$$

It is clear that the composition above gives the identity map on $\mathbb{C}\left[\mathfrak{h} \times \mathfrak{h}^{*}\right] \# W$. It follows that the map $\operatorname{gr}_{F}\left(\widetilde{\Theta}_{t, c}\right)$, hence $\widetilde{\Theta}_{t, c}$, is injective. Furthermore, we deduce that $\operatorname{gr}_{F}\left(\mathrm{H}_{t, c}\right) \simeq$ $\mathbb{C}\left[\mathfrak{h} \times \mathfrak{h}^{*}\right] \# W$.

The definition of Dunkl operators as well as the Proposition above can be extended easily to the case of any complex reflection group $W$, see $[\mathrm{BMR}]$. In particular, we get the following result, whose verification by (quite non-trivial) direct computations is first due to Dunkl [D1],[D2] and Cherednik [Ch] in the Weyl group case, and in [BMR] in general (for complex reflection groups).

Corollary 4.6 The Dunkl operators $D_{y}, y \in \mathfrak{h}$, commute with each other.

Following Olshanetsky-Perelomov [OP], define the rational Calogero-Moser operator with parameters $\kappa=(t, c) \in \overline{\mathrm{C}}$, to be the following $W$-invariant differential operator on $\mathfrak{h}^{\text {reg }}$ :

$$
\mathrm{L}_{\kappa}\left(=\mathrm{L}_{t, c}\right)=t^{2} \cdot \Delta_{\mathfrak{h}}-\frac{1}{2} \sum_{\alpha \in R} c_{\alpha}\left(c_{\alpha}+t\right) \cdot \frac{(\alpha, \alpha)}{\alpha^{2}} \quad \text { (Calogero-Moser operator). }
$$

From now on we fix $c \in \mathrm{C}$, and set $\delta_{c}=\prod_{\alpha \in R} \alpha^{c_{\alpha} / 2}$. For $t \in \mathbb{C}$, we consider the modified homomorphism $\Theta_{t, c}:=\frac{1}{\delta_{c / t}} \circ \widetilde{\Theta}_{t, c} \circ \delta_{c / t}: \mathrm{H}_{t, c} \longrightarrow \mathcal{D}\left(\mathfrak{h}^{\mathrm{reg}}\right) \# W$, sending $u \in \mathrm{H}_{t, c}$ to the operator: $f \mapsto \frac{1}{\delta_{c / t}} \cdot \widetilde{\Theta}_{t, c}(u)\left(\delta_{c / t} \cdot f\right)$. Although $\delta_{c / t}$ is a multivalued function on $\mathfrak{h}$, the homomorphism $\frac{1}{\delta_{c / t}} \circ \widetilde{\Theta}_{c / t} \circ \delta_{c / t}$ is well-defined on generators, hence, on the whole of $\mathrm{H}_{\kappa}$. Thus, Proposition 4.5 implies that $\Theta_{t, c}: \mathrm{H}_{t, c} \longrightarrow \mathcal{D}\left(\mathfrak{h}^{\mathrm{reg}}\right) \# W$ is an injective algebra homomorphism. The restriction of $\Theta_{t, c}$ to the spherical subalgebra gives an algebra homomorphism

$$
\Theta_{\kappa}^{\text {spher }}: \mathbf{e H}_{\kappa} \mathbf{e} \longrightarrow \mathbf{e}\left(\mathcal{D}\left(\mathfrak{h}^{\text {reg }}\right) \# W\right) \mathbf{e}=\mathcal{D}\left(\mathfrak{h}^{\text {reg }}\right)^{W}
$$


see (2.15). The map $\Theta_{\kappa}^{\text {spher }}$ clearly respects the filtrations on both sides. Furthermore, a straighforward calculation yields: $\Theta_{\kappa}^{\text {spher }}\left(\mathbf{e} \cdot \Delta_{\mathfrak{h}}\right)=\mathrm{L}_{\kappa}$, where $\Delta_{\mathfrak{h}} \in S \mathfrak{h}^{W}=\mathbb{C}\left[h^{*}\right]^{W}$ is the quadratic Casimir, viewed as an element of $\mathbb{C}\left[h^{*}\right]^{W} \subset \mathrm{H}_{\kappa}$ (note that $\mathbf{e} \cdot \Delta_{\mathfrak{h}}=\Delta_{\mathfrak{h}} \cdot \mathbf{e}$ ).

Let $\mathcal{C}_{t, c}$ be the centraliser of $\mathrm{L}_{t, c}$ in $\mathcal{D}\left(\mathfrak{h}^{\text {reg }}\right)_{-}^{W}$ (= the algebra spanned by homogeneous elements $D \in \mathcal{D}\left(\mathfrak{h}^{\text {reg }}\right)^{W}$ such that $\left.\operatorname{order}(D)+\operatorname{degree}(D) \leq 0\right)$. Let $\mathcal{B}_{t, c}$ be the subalgebra in $\mathcal{D}\left(\mathfrak{h}^{\text {reg }}\right)^{W}$ generated by $\mathcal{C}_{t, c}$ and $\mathbb{C}[\mathfrak{h}]^{W} \subset \mathcal{D}(\mathfrak{h})^{W}$, the subalgebra of $W$-invariant polynomials.

Theorem 4.8 (i) For any fixed $c \in \mathrm{C}$, and generic $t \neq 0$ (i.e. outside of a countable set), we have: $\operatorname{Im}\left(\Theta_{t, c}^{\text {spher }}\right)=\mathcal{B}_{t, c}$.

(ii) If, for some $(t, c) \in \mathbb{C} \oplus \mathrm{C}$, the image of $\Theta_{t, c}^{\mathrm{spher}}$ is contained in $\mathcal{B}_{t, c}$ then the following maps are algebra isomorphisms

$$
\Theta_{t, c}^{\text {spher }}: \mathbf{e H}_{t, c} \mathbf{e} \stackrel{\sim}{\longrightarrow} \mathcal{B}_{t, c}, \quad \text { and } \quad \operatorname{gr}\left(\Theta_{t, c}^{\text {spher }}\right): \operatorname{gr}\left(\mathbf{e H}_{t, c} \mathbf{e}\right) \stackrel{\sim}{\longrightarrow} \operatorname{gr}\left(\mathcal{B}_{t, c}\right) .
$$

Proof: (i) For generic $t$, the algebra $\mathbf{e H}_{\kappa} \mathbf{e}$ is generated, as an associative algebra, by the subspaces: $\mathbb{C}[\mathfrak{h}]^{W}$.e and $\mathbb{C}\left[\mathfrak{h}^{*}\right]^{W} \cdot \mathbf{e}$. Indeed, this is known [LS1 to be true in the Weyl algebra case: $t=1, c=0$, hence it is true for all $t$ except a countable set.

On the other hand, the image of $\mathbb{C}[\mathfrak{h}]^{W}$.e under the map $\Theta_{t, c}^{\text {spher }}$ equals $\mathbb{C}[\mathfrak{h}]^{W}$. Also, the results of Opdam (see $\left[\mathrm{O}\right.$, , [Ki]) imply that the image of $\mathbb{C}\left[\mathfrak{h}^{*}\right]^{W} \cdot \mathbf{e}$ under this map is the algebra $\mathcal{C}_{\kappa}$. This implies (i).

(ii) In the proof of (i) we showed that $\mathcal{B}_{\kappa}$ is always contained in the image of $\Theta_{t, c}^{\text {spher }}$ Thus, under our assumptions, the image of $\Theta_{\kappa}^{\text {spher }}$ equals $\mathcal{B}_{\kappa}$. We know that the map $\operatorname{gr}\left(\Theta_{\kappa}^{\text {spher }}\right)$ is injective (it is independent on $\left.\kappa\right)$. Therefore, the map $\Theta_{\kappa}^{\text {spher }}$ is also injective. We conclude that $\Theta_{\kappa}^{\text {spher }}: \mathbf{e H}_{\kappa} \mathbf{e} \rightarrow \mathcal{B}_{\kappa}$ is an isomorphism.

It remains to show that $\operatorname{gr}\left(\Theta_{\kappa}^{\text {spher }}\right): \operatorname{gr}\left(\mathbf{e H}_{\kappa} \mathbf{e}\right) \rightarrow \operatorname{gr}\left(\mathcal{B}_{\kappa}\right)$ is an isomorphism. But this is clear, since if $f$ is any filtered map of $\mathbb{Z}_{+}$-graded spaces such that $\operatorname{gr}(f)$ is injective and $f$ is an isomorphism then $\operatorname{gr}(f)$ is also an isomorphism. We are done.

Proposition 4.9 Suppose that $W$ is a Weyl group with no factors of type $\mathbf{E}$ or $\mathbf{F}$. Then part (i) of Theorem 4.8, in particular, the conclusion of part (ii), holds for all values of $t$.

Proof. We need to show that the image of $\Theta_{\kappa}^{\text {spher }}$ is contained in $\mathcal{B}_{t, c}$. For this, it is sufficient to prove that $\mathbf{e H}_{t, c} \mathbf{e}$ is generated, as an algebra, by the subalgebras $\mathbb{C}[\mathfrak{h}]^{W}$ and $\mathbb{C}\left[\mathfrak{h}^{*}\right]^{W}$. This follows from a stronger statement that $\operatorname{gr}\left(\mathbf{e} \mathrm{H}_{t, c} \mathbf{e}\right)=\mathbb{C}\left[\mathfrak{h} \oplus \mathfrak{h}^{*}\right]^{W}$ is generated, as a Poisson algebra, by the subalgebras $\mathbb{C}[\mathfrak{h}]^{W}$ and $\mathbb{C}\left[\mathfrak{h}^{*}\right]^{W}$. But this is shown in Wa for Weyl groups containing no factors of type $\mathbf{E}$ or $\mathbf{F}$.

We expect, but cannot show, that the same result holds for all finite reflection groups.

Relation of $\mathrm{H}_{\kappa}$ to the double-affine Hecke algebra. The algebra $\mathrm{H}_{\kappa}$ is a rational version of the double-affine Hecke algebra. By this we mean that if $W$ is the Weyl group associated to a finite root system $R$, then $\mathrm{H}_{\kappa}$ is obtained from the double affine Hecke algebra attached to $W$ by a certain limiting procedure. 
To illustrate this, we will consider the special case of the root system of type $\mathbf{A}_{\mathbf{1}}$. In this case $W=\{1, s\}$. Thus, if $t=1$ and $c=c_{\alpha}$, for the unique positive root $\alpha$, then, the algebra $\mathrm{H}_{1, c}$ is generated by 3 elements: $s, x, y$, with defining relations

$$
s^{2}=1 \quad, \quad s x=-x s \quad, \quad s y=-y s \quad, \quad[y, x]=1-2 c \cdot s .
$$

Let us show how the algebra $\mathrm{H}_{1, c}$ is obtained from the double-affine Hecke algebra by a limiting procedure. Let us consider the version of $\mathrm{H}_{1, c}$ over $\mathbb{C}[c]$, which we will denote in the same way. Recall that the double-affine Hecke algebra $\mathbf{H}$ is the $\mathbb{C}\left[q^{ \pm 1}, \tau^{ \pm 1}\right]$-algebra with 3 generators: $T, X, Y$ (where $X, Y$ are invertible), and defining relations:

$$
(T-\tau)\left(T+\tau^{-1}\right)=0, T X T=X^{-1}, T^{-1} Y T^{-1}=Y^{-1}, Y^{-1} X^{-1} Y X T^{2}=q .
$$

Define a completed double-affine Hecke algebra $\widehat{\mathbf{H}}$ to be a $\mathbb{C}[c][[h]]$-algebra topologically generated (in the $h$-adic topology) by $s, x, y$, with the same defining relations, for $X=e^{h x}$, $Y=e^{h y}, T=s e^{h^{2} c s}, q=e^{h^{2}}, \tau=e^{h^{2} c}$. It is clear that $\widehat{\mathbf{H}}$ is a certain completion of $\mathbf{H}$.

The proposition below says that the algebra $\widehat{\mathbf{H}}$ is a flat deformation of $\mathbf{H}_{1, c}$.

Proposition 4.10 The algebra $\widehat{\mathbf{H}}$ is flat over $\mathbb{C}[[h]]$, and $\widehat{\mathbf{H}} / h \cdot \widehat{\mathbf{H}}=\mathrm{H}_{1, c}$.

Proof. The last statement is obtained directly from the relations: one shows that the relations of $\widehat{\mathbf{H}}$ are deformations of the relations of $\mathrm{H}_{1, c}$. The flatness statement follows from the fact, due to Cherednik, that the representation of $\mathrm{H}_{1, c}$ by Dunkl operators can be deformed to a faithful representation of $\widehat{\mathbf{H}}$ by q-difference analogs of Dunkl operators.

A similar relation between $\mathrm{H}_{\kappa}$ and the double-affine Hecke algebra exists for any Weyl group. The statement and proof of this fact is analogous to the $\mathfrak{s l}_{2}$ case.

Fourier transform. There is an important algebra automorphism $\mathrm{F}: \mathrm{H}_{\kappa} \rightarrow \mathrm{H}_{\kappa}$, to be called the Fourier transform. The automorphism $\mathrm{F}$ is a rational version of Cherednik's "difference Fourier transform" for double affine Hecke algebras. In particular, in the $\mathfrak{s l}_{2}$ case, it is the classical Fourier-Hankel transform, see ChM.

Let $(-,-)$ be the $W$-invariant bilinear form on $\mathfrak{h}$ that has been fixed throughout. For $x \in \mathfrak{h}^{*}$, let $\check{x}$ be the element of $\mathfrak{h}$ such that $x=(\check{x},-)$, and for $y \in \mathfrak{h}$, let $\check{y}=(y,-)$ be the corresponding element of $\mathfrak{h}^{*}$. The Fourier transform on $\boldsymbol{H}_{\kappa}$ is defined to be an algebra homomorphism $\mathrm{F}: \mathrm{H}_{\kappa} \rightarrow \mathrm{H}_{\kappa}$ given on generators by the following assignment:

$$
\mathrm{F}: x \mapsto \check{x} \quad, \quad y \mapsto-\check{y} \quad, \quad w \mapsto w, \quad \forall x \in \mathfrak{h}^{*}, y \in \mathfrak{h}, w \in W .
$$

It is clear that $\mathrm{F}$ extends to an automorphism of $\mathrm{H}_{\kappa}$ preserving the subalgebra $\mathbf{e H}_{\kappa} \mathbf{e}$. It follows that (in cases when $\mathbf{e H}_{\kappa} \mathbf{e}$ is isomorphic to $\mathcal{B}_{\kappa}$, see Theorem 4.8 and Proposition 4.9) it also acts on $\mathcal{B}_{\kappa}$, permuting $\mathbb{C}[\mathfrak{h}]^{W}$ and $\mathcal{C}_{\kappa}$. In particular, this is so for type $\mathbf{A}_{\mathbf{n}}$.

Homomorphism $\Theta_{\mathbf{0}, \mathbf{c}}$. We now fix $c \in \mathbf{C}, c \neq 0$, and consider the limit of the map $\Theta_{t, c}$, see above (4.7), when $t \rightarrow 0$. Let $\mathcal{D}_{t}(\mathfrak{h})$ be the Weyl algebra of the vector space $\mathfrak{h} \oplus \mathfrak{h}^{*}$ equipped with the standard symplectic form multiplied by the parameter $t$. Thus, the algebra $\mathcal{D}_{t}(\mathfrak{h})$ is generated by $\mathfrak{h}$ and $\mathfrak{h}^{*}$, so that elements of $\mathfrak{h}$ commute, elements of 
$\mathfrak{h}^{*}$ commute, and we have: $[y, x]=t \cdot\langle x, y\rangle, \forall x \in \mathfrak{h}^{*}, y \in \mathfrak{h}$. Let $\mathcal{D}_{t}\left(\mathfrak{h}^{\mathrm{reg}}\right)$ be the localized algebra obtained by inverting the Weyl denominator $\delta_{x} \in \mathbb{C}[\mathfrak{h}] \subset \mathcal{D}_{t}(\mathfrak{h})$. For $t \neq 0$, the assignment: $y \mapsto t \partial_{y}, x \mapsto x$ gives an algebra isomorphism $\phi_{t}: \mathcal{D}_{t}\left(\mathfrak{h}^{\text {reg }}\right) \stackrel{\sim}{\longrightarrow} \mathcal{D}\left(\mathfrak{h}^{\text {reg }}\right)$. For $t \neq 0$, the composite map $\Theta_{t, c}^{\sharp}=\left(\phi_{t}\right)^{-1} \circ \Theta_{t, c}: \mathrm{H}_{t, c} \longrightarrow \mathcal{D}_{t}\left(\mathfrak{h}^{\text {reg }}\right) \# W$ is the identity map on $W$, and is given on generators: $x \in \mathfrak{h}^{*}, y \in \mathfrak{h}$, by the following formulas:

$$
x \mapsto x \quad, \quad y \mapsto \frac{1}{\delta_{c / t}} \cdot y \cdot \delta_{c / t}+\frac{1}{2} \sum_{\alpha \in R} c_{\alpha} \cdot \frac{\langle\alpha, y\rangle}{\alpha}\left(s_{\alpha}-1\right)=y+\frac{1}{2} \sum_{\alpha \in R} c_{\alpha} \cdot \frac{\langle\alpha, y\rangle}{\alpha} s_{\alpha} .
$$

Since the formulas above do not involve the parameter $t$, the homomorphism $\Theta_{t, c}^{\sharp}$ makes sense for $t=0$, and we set $\Theta_{0, c}:=\Theta_{0, c}^{\sharp}$.

In the case $t=0$ we have: $\mathcal{D}_{t}\left(\mathfrak{h}^{\text {reg }}\right)=\mathbb{C}\left[\mathfrak{h}^{\text {reg }} \times \mathfrak{h}^{*}\right]$, a commutative algebra. Copying the proof of theorem 4.8 one obtains:

Proposition 4.11 The map $\Theta_{0, c}: \mathrm{H}_{0, c} \longrightarrow \mathbb{C}\left[\mathfrak{h}^{\mathrm{reg}} \times \mathfrak{h}^{*}\right] \# W$ is an injective algebra homomorphism.

Write: $p \mapsto|p|^{2}$ for the quadratic polynomial on $\mathfrak{h}^{*}$ corresponding to the squared norm relative to the invariant form. View it as an element of $\mathbb{C}\left[\mathfrak{h}^{\text {reg }} \times \mathfrak{h}^{*}\right]$ constant along the first factor, and view $\alpha \in R$ as a function on $\mathfrak{h}^{\text {reg }} \times \mathfrak{h}^{*}$ constant along the second factor.

Definition 4.12 ( $\mathrm{OP})$ The function $\mathrm{L}_{0, c}:(x, p) \mapsto|p|^{2}-\frac{1}{2} \sum_{\alpha \in R} \frac{c_{\alpha}\left(c_{\alpha}+1\right)}{\alpha(x)^{2}}$, on $\mathfrak{h}^{\mathrm{reg}} \times \mathfrak{h}^{*}$, is called the (classical) Calogero-Moser hamiltonian.

A straightforward calculation yields: $\Theta_{0, c}^{\text {spher }}\left(\mathbf{e} \cdot|p|^{2}\right)=\mathrm{L}_{0, c}$, where $|p|^{2}$ denotes the element of $\mathrm{H}_{0, c}$ corresponding to the polynomial: $p \mapsto|p|^{2}$, viewed as an element of $\mathbb{C}\left[\mathfrak{h}^{*}\right]^{W} \subset \mathrm{H}_{0, c}$.

Further, the standard symplectic structure on $T^{*}\left(\mathfrak{h}^{\text {reg }}\right)=\mathfrak{h}^{\text {reg }} \times \mathfrak{h}^{*}$ makes $\mathbb{C}\left[\mathfrak{h}^{\text {reg }} \times \mathfrak{h}^{*}\right]$ a Poisson algebra. We have: $\mathfrak{L}_{0, c} \in \mathbb{C}\left[\mathfrak{h}^{\text {reg }} \times \mathfrak{h}^{*}\right]^{W}$. Let $\mathbb{C}\left[\mathfrak{h}^{\text {reg }} \times \mathfrak{h}^{*}\right]_{-}^{W}$ denote the algebra spanned by bi-homogeneous functions $P \in \mathbb{C}\left[\mathfrak{h}^{\text {reg }} \times \mathfrak{h}^{*}\right]^{W}$ such that total degree $(P)+p$-degree $(P) \leq 0$. Let $\mathcal{C}_{0, c}$ denote the centralizer in $\mathbb{C}\left[\mathfrak{h}^{\text {reg }} \times \mathfrak{h}^{*}\right]_{-}^{W}$ of the Calogero-Moser hamiltonian $\mathrm{L}_{0, c}$ with respect to the Poisson bracket, and let $\mathcal{B}_{0, c}$ denote the Poisson subalgebra of $\mathbb{C}\left[\mathfrak{h}^{\text {reg }} \times \mathfrak{h}^{*}\right]^{W}$ generated by $\mathcal{C}_{0, c}$ and the algebra $\mathbb{C}\left[\mathfrak{h}^{*}\right]^{W}$. On the other hand, recall that the spherical subalgebra $\mathbf{e H}_{0, c} \mathbf{e}$ has a natural Poisson algebra structure defined using the deformation construction of $\S 15$.

Proposition 4.13 If $W$ is a Weyl group which has no factors of type $\mathbf{E}$ or $\mathbf{F}$, then the map $\Theta_{0, c}$ induces a Poisson algebra isomorphism $\Theta_{0, c}^{\text {spher }}: \mathbf{e H}_{0, c} \mathbf{e} \stackrel{\sim}{\longrightarrow} \mathcal{B}_{0, c}$. Moreover, the associated graded map $\operatorname{gr}\left(\Theta_{0, c}^{\text {spher }}\right): \operatorname{gr}\left(\mathbf{e H}_{0, c} \mathbf{e}\right) \stackrel{\sim}{\longrightarrow} \operatorname{gr}\left(\mathcal{B}_{0, c}\right)$ is also bijective.

Proof. Since $\Theta_{0, c}^{\text {spher }}$ is the specialisation at $t=0$ of the family of associative algebra homomorphisms $\left\{\Theta_{t, c}^{\text {spher }}\right\}_{t \neq 0}$, general 'deformation formalism' implies that $\Theta_{0, c}^{\text {spher }}$ is a 
homomorphism of Poisson algebras. The rest of the argument is identical to that in the proof of Theorem 4.8 and Proposition 4.9 .

Since $\operatorname{gr}\left(Z_{0, c}\right)=Z_{0,0}$ is a finitely generated algebra without zero divisors, it follows that $Z_{0, c}$ is a finitely generated algebra without zero divisors as well. Therefore, it defines an irreducible affine algebraic variety $\operatorname{Spec} Z_{0, c}$ of $\operatorname{dimension}$ equal to $\operatorname{dim} V$. This variety has a canonical Poisson structure. Thus, $\operatorname{Spec} Z_{0, c}$ is a Poisson deformation of the Poisson variety $V^{*} / W$, cf. also $\S 17$. We propose the following

Definition 4.14 The Poisson variety $\operatorname{Spec} \mathrm{Z}\left(\mathrm{H}_{0, c}\right)$ is called the Calogero-Moser space associated to $W$, and the parameter $c \in \mathrm{C}$.

The variety $\operatorname{Spec} \mathrm{Z}\left(\mathrm{H}_{0, c}\right)$ may be regarded as the "correct" completion of the phase space for the classical Calogero-Moser system acssociated to the Weyl group $W$. We have shown that $\operatorname{Spec} Z\left(\mathrm{H}_{0, c}\right)$ is an irreducible Gorenstein normal variety. The problem of smoothness of $\operatorname{Spec} Z\left(\mathrm{H}_{0, c}\right)$ will be considered, in a few special cases, in Appendix E.

We now study the center of the algebra $\mathrm{H}_{0, c}$ in some detail.

Proposition 4.15 For an arbitrary finite complex reflection group $W$, we have:

(i) The subalgebras $\mathbb{C}\left[\mathfrak{h}^{*}\right]^{W} \subset \mathrm{H}_{0, c}$ and $\mathbb{C}[\mathfrak{h}]^{W} \subset \mathrm{H}_{0, c}$ are both contained in $\mathrm{Z}_{0, c}$.

(ii) The algebra $\mathrm{Z}_{0, c}$ is a free $\mathbb{C}[\mathfrak{h}]^{W} \otimes \mathbb{C}\left[\mathfrak{h}^{*}\right]^{W}$-module of rank $|W|$.

Proof. The inclusion: $\mathbb{C}\left[\mathfrak{h}^{*}\right]^{W} \subset \mathrm{Z}_{0, c}$ follows from the injectivity of $\Theta_{0, c}$ and the fact that, for $f \in \mathbb{C}\left[\mathfrak{h}^{*}\right]^{W}$, we have $\Theta_{0, c}(f) \in \mathbb{C}\left[\mathfrak{h}^{\text {reg }}\right]^{W}$, which is obviously in the center of $\mathbb{C}\left[\mathfrak{h}^{\text {reg }} \times \mathfrak{h}^{*}\right] \# W$. The inclusion: $\mathbb{C}[\mathfrak{h}]^{W} \subset \mathrm{Z}_{0, c}$ follows from the previous one by applying the Fourier automorphism F. Thus, we have shown that: $\mathbb{C}[\mathfrak{h}]^{W} \otimes \mathbb{C}\left[\mathfrak{h}^{*}\right]^{W} \subset \mathrm{Z}_{0, c}$.

Further, $\mathbb{C}\left[\mathfrak{h} \oplus \mathfrak{h}^{*}\right]$ is a free module over its subalgebra $\mathbb{C}[\mathfrak{h}]^{W} \otimes \mathbb{C}\left[\mathfrak{h}^{*}\right]^{W} \subset \mathbb{C}\left[\mathfrak{h} \oplus \mathfrak{h}^{*}\right]$, by the Chevalley theorem. Moreover, one has a $W \times W$-module isomorphism:

$$
\mathbb{C}\left[\mathfrak{h} \oplus \mathfrak{h}^{*}\right] \simeq \mathbb{C}[W \times W] \bigotimes\left(\mathbb{C}[\mathfrak{h}]^{W} \otimes \mathbb{C}\left[\mathfrak{h}^{*}\right]^{W}\right)
$$

Taking the invariants with respect to the diagonal subgroup $W \subset W \times W$ shows that $\mathbb{C}\left[\mathfrak{h} \oplus \mathfrak{h}^{*}\right]^{W}$ is a free $\mathbb{C}[\mathfrak{h}]^{W} \otimes \mathbb{C}\left[\mathfrak{h}^{*}\right]^{W}$-module of rank $|W|$. Part (ii) now follows from the isomorphism $\operatorname{gr}\left(\mathrm{Z}_{0, c}\right) \stackrel{\sim}{\longrightarrow} \mathbb{C}\left[\mathfrak{h} \oplus \mathfrak{h}^{*}\right]^{W}$ of Theorem 3.3.

The imbedding: $\mathbb{C}[\mathfrak{h}]^{W} \otimes \mathbb{C}\left[\mathfrak{h}^{*}\right]^{W} \hookrightarrow \mathrm{Z}_{0, c}$ of Proposition 4.15 gives rise to a finite surjective map $\Upsilon: \operatorname{Spec} Z_{0, c} \rightarrow \mathfrak{h} / W \times \mathfrak{h}^{*} / W$, of degree $|W|$. Let $\mid W /$ Ad $\mid$ denote the number of conjugacy classes in the finite complex reflection group $W$.

Proposition 4.16 If Spec $Z_{0, c}$ is smooth, then the fiber $\Upsilon^{-1}(0) \subset$ Spec $Z_{0, c}$ consists of $|W / \operatorname{Ad}|$ points. 
Proof. The $\mathbb{C}^{*}$-action on $\mathfrak{h} \oplus \mathfrak{h}^{*}$ given by the assignment $\mathbb{C}^{*} \ni \lambda: y \oplus x \mapsto \lambda \cdot y \oplus \lambda^{-1} \cdot x$ extends to a $\mathbb{C}^{*}$-action on $\mathrm{H}_{t, c}$ by algebra automorphisms keeping the group $W$ pointwise fixed (this is part of the $S L_{2}(\mathbb{C})$-action on $\mathrm{H}_{t, c}$ considered in Corollary 5.3). The $\mathbb{C}^{*}$-action on $\mathrm{H}_{0, c}$ induces an action on $\mathrm{Z}_{0, c}$, hence on Spec $\mathrm{Z}_{0, c}$.

Furthermore, the $\mathbb{C}^{*}$-action on $\mathfrak{h} \oplus \mathfrak{h}^{*}$ induces one on $\mathfrak{h} / W \times \mathfrak{h}^{*} / W$, so that the map $\Upsilon$ becomes $\mathbb{C}^{*}$-equivariant. Observe that the point $(0,0) \in \mathfrak{h} / W \times \mathfrak{h}^{*} / W$ is the only fixed point of the $\mathbb{C}^{*}$-action on $\mathfrak{h} / W \times \mathfrak{h}^{*} / W$. It follows, since $\mathbb{C}^{*}$ is connected and the fiber $\Upsilon^{-1}(0)$ is finite, that each point of $\Upsilon^{-1}(0)$ is a $\mathbb{C}^{*}$-fixed point, and these are the only fixed points on $\operatorname{Spec} Z_{0, c}$.

Now, the Lefschetz fixed point formula for the $\mathbb{C}^{*}$-action on Spec $Z_{0, c}$ yields: $\left|\Upsilon^{-1}(0)\right|=$ $\chi\left(\operatorname{Spec} Z_{0, c}\right)$, where $\chi(-)$ stands for the Euler characteristic. On the other hand, if Spec $Z_{0, c}$ is smooth, then from Theorem 1.8 one finds: $\chi\left(\operatorname{Spec} Z_{0, c}\right)=\mid W /$ Ad $\mid$. The result follows.

Simple $\mathrm{H}_{0, c^{-}}$modules correspoding to points of $\Upsilon^{-1}(0)$ may be called nilpotent, because they are characterized by the property that all elements of both $\mathfrak{h} \subset \mathrm{H}_{0, c}$ and $\mathfrak{h}^{*} \subset \mathrm{H}_{0, c}$ act by nilpotent operators in them.

Remark. For any finite Coxeter group $W$, results similar to Theorem 1.5 are valid if the symmetrizer $\mathbf{e}$ is replaced with the antisymmetrizer $\mathbf{e}_{-}=\frac{1}{|W|} \sum_{w \in W}(-1)^{\ell(w)} \cdot w$, since there is an anti-involution of $\mathrm{H}_{0, c}$ that acts trivially on $\mathfrak{h} \oplus \mathfrak{h}^{*}$ and acts on $W$ via: $w \mapsto(-1)^{\ell(w)} \cdot w$; this anti-involution interchanges $\mathbf{e}$ and $\mathbf{e}_{-} . \diamond$

Partial brackets. A slight generalization of the construction of the Poisson brackets on commutative algebras $\mathbf{e H}_{0, c} \mathbf{e}$ and $\mathrm{Z}_{0, c}$ (see $\S 15$ ) can be also applied to construct bilinear pairings:

$$
\mathbb{C}[\mathfrak{h}]^{W} \times \mathrm{H}_{0, c} \longrightarrow \mathrm{H}_{0, c} \quad \text { and } \quad \mathbb{C}\left[\mathfrak{h}^{*}\right]^{W} \times \mathrm{H}_{0, c} \longrightarrow \mathrm{H}_{0, c} \quad, \quad(P, u) \mapsto\{P, u\} .
$$

To define these pairings, observe that, for any $(t, c) \in \mathbb{C} \oplus \mathrm{C}$, one has a canonical algebra imbedding $\epsilon_{t, c}: \mathbb{C}[\mathfrak{h}]^{W} \hookrightarrow \mathrm{H}_{t, c}$ and, moreover, the algebra $\epsilon_{0, c}\left(\mathbb{C}[\mathfrak{h}]^{W}\right)$ is central in $\mathrm{H}_{0, c}$. Now, given an element $u \in \mathrm{H}_{0, c}$ choose an algebraic family $u_{t} \in \mathrm{H}_{t, c}$ such that $u_{0, c}=u$. For any $z \in \mathbb{C}[\mathfrak{h}]^{W}$ define an element $\{z, u\} \in \mathrm{H}_{0, c}$ to be the linear term in the expansion of $\left[\epsilon_{t, c}(z), u_{t}\right]=\epsilon_{t, c}(z) \cdot u_{t}-u_{t} \cdot \epsilon_{t, c}(z)$ at $t=0$. This term is independent of the choice of the family $u_{t} \in \mathrm{H}_{t, c}$ such that $u_{t}=u$, since $\epsilon_{0, c}(z)$ is a central element. For each $z \in \mathbb{C}[\mathfrak{h}]^{W}$, the assignment: $u \mapsto\{z, u\}$ gives a derivation of the algebra $\mathrm{H}_{0, c}$, which reduces, on $\mathrm{Z}_{0, c}$, to the map given by taking the Poisson bracket (in $\mathrm{Z}_{0, c}$ ) with $\epsilon_{0, c}(z)$.

A similar construction applies to the algebra $\mathbb{C}\left[\mathfrak{h}^{*}\right]^{W}$ instead of $\mathbb{C}[\mathfrak{h}]^{W}$. The reader should be warned that we have not constructed a 'Poisson bracket': $\mathbf{Z}_{0, c} \times \mathrm{H}_{0, c} \rightarrow \mathrm{H}_{0, c}$ since, for $t \neq 0$, there is no obvious family of vector space imbeddings $\epsilon_{t, c}: Z_{0, c} \hookrightarrow H_{t, c}$.

\section{Automorphisms and derivations of the algebra $\mathrm{H}_{\kappa}$.}

Let $(\mathfrak{h}, W, R)$ be as in $\S 4$. In Ch2, Cherednik made an observation, equivalent to 
Proposition 5.1 Let $f \in \mathbb{C}[\mathfrak{h}]^{W}$. Then, for any $t \neq 0$, the assignment

$$
w \mapsto w \quad, \quad x \mapsto x \quad, \quad y \mapsto y-\frac{\partial f(x)}{\partial x} \quad, \quad x \in \mathfrak{h}^{*}, w \in W, y \in \mathfrak{h}
$$

uniquely extends to an automorphism $a_{f}: \mathrm{H}_{t, c} \rightarrow \mathrm{H}_{t, c}$, acting trivially on $\mathbb{C} W \subset \mathrm{H}_{t, c}$. $A$ similar result holds for $f \in \mathbb{C}\left[\mathfrak{h}^{*}\right]^{W}$.

Proof. It is easy to see that, for any $u \in \mathcal{D}\left(\mathfrak{h}^{\text {reg }}\right) \# W$, the operator $e^{-f} \circ u \circ e^{f}$ is again

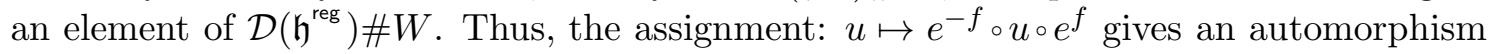
of the algebra $\mathcal{D}\left(\mathfrak{h}^{\text {reg }}\right) \# W$. We transport this automorphism to $\mathrm{H}_{t, c}$ via the (faithful) representation of $\Theta_{t, c}: \mathrm{H}_{t, c} \rightarrow \mathcal{D}\left(\mathfrak{h}^{\text {reg }}\right) \# W$ by Dunkl operators. A straightforward calculation shows that the transported automorphism is given, on generators, by the formulas of the Proposition, and in particular, maps $\operatorname{Im}\left(\Theta_{t, c}\right)$ to itself. The proposition is proved.

Remark. The automorphism $a_{f}$ can be informally written as " $\operatorname{Ad}\left(e^{f}\right)$ ", i.e. $a_{f}$ is, essentially, an "inner" automorphism induced by the element $e^{f}$ (which belongs to an appropriate completion of $\left.\mathrm{H}_{t, c}\right)$. $\diamond$

Corollary 5.2 The assignment of Proposition 5.1 uniquely extends to an automorphism $a_{f}: \mathrm{H}_{0, c} \rightarrow \mathrm{H}_{0, c}$.

Proof. Since the relations (1.15) among the generators of the algebras $\mathrm{H}_{t, c}$ depend on $t$ in a continuous fashion, and the assignment of Proposition 5.1 is independent of the parameter $t$, the result follows from Proposition 5.1 'by continuity'.

Write $\check{x}$, resp. $\check{y}$, for the element of $\mathfrak{h}$ corresponding to $x \in \mathfrak{h}^{*}$, resp. the element of $\mathfrak{h}^{*}$ corresponding to $y \in \mathfrak{h}$, under the invariant form $(-,-)$ on $\mathfrak{h}$. The following result is immediate.

Corollary 5.3 The assignment: $x \rightarrow a x+b \check{y}, \quad y \rightarrow c \check{x}+d y, \quad(a d-b c=1)$, extends to an $S L_{2}(\mathbb{C})$-action on each algebra $\mathrm{H}_{\kappa}, \kappa \in \overline{\mathrm{C}}$, by algebra automorphisms.

The above results can be applied to study derivations of the algebra $\mathrm{H}_{\kappa}$, viewed as 'infinitesimal' automorphisms of $\mathbf{H}_{\kappa}$. Fix $f \in \mathbb{C}[\mathfrak{h}]^{W}$, and consider the one-parameter group: $\tau \mapsto a_{\tau \cdot f}, \tau \in \mathbb{C}$, of automorphisms of the algebra $\mathrm{H}_{\kappa}$. Taking the derivative of this family at $\tau=0$ we conclude that the map $\theta_{f}:=\left.\frac{d a_{\tau \cdot f}}{d \tau}\right|_{\tau=0}: \mathrm{H}_{\kappa} \rightarrow \mathrm{H}_{\kappa}$ is a derivation. Formulas of Proposition 5.1 yield:

$$
\theta_{f}: \quad w \mapsto 0 \quad, \quad x \mapsto 0 \quad, \quad y \mapsto-\frac{\partial f(x)}{\partial x} \quad, \quad x \in \mathfrak{h}^{*}, w \in W, y \in \mathfrak{h} .
$$

Note that, if $\kappa=(t, c)$ where $t \neq 0$, we have: $\theta_{f}=\operatorname{ad} f$, i.e., $\theta_{f}$ is an inner derivation. For $\kappa=(0, c)$, the derivation $\theta_{f}$ is not inner. It is clearly obtained from a family of inner derivations on the family of algebras $\mathrm{H}_{t, c}, t \neq 0$, by a "limiting procedure" as $t \rightarrow 0$. This argument yields the following expression for $\theta_{f}$ in terms of the 'partial bracket' $\{-,-\}: \mathbb{C}[\mathfrak{h}]^{W} \times \mathrm{H}_{0, c} \rightarrow \mathrm{H}_{0, c}$, see (4.17). 
Proposition 5.5 For any $f \in \mathbb{C}[\mathfrak{h}]^{W}$, resp. $f \in \mathbb{C}\left[\mathfrak{h}^{*}\right]^{W}$, in $\mathrm{H}_{0, c}$ one has: $\theta_{f}(u)=$ $\{f, u\}, \forall u \in \mathrm{H}_{0, c}$.

The $\mathfrak{g l}_{n}$-case. From now until the end of this section, let $R$ be the root system of type $\mathbf{A}_{\mathbf{n}-\mathbf{1}}$ in the vector space $\mathfrak{h}=\mathbb{C}^{n}$ (not in $\mathbb{C}^{n-1}$ ). Write $x_{1}, \ldots, x_{n}$, for the standard coordinates on $\mathfrak{h}=\mathbb{C}^{n}$, and $y_{1}, \ldots, y_{n}$, for the dual coordinates on $\mathfrak{h}^{*}$. We introduce the following "power sum" elements:

$$
\psi_{\ell}=\sum_{i=1}^{n} x_{i}^{\ell} \in \mathbb{C}\left[\mathfrak{h}^{*}\right]^{W} \quad \text { and } \quad \phi_{\ell}=\sum_{i=1}^{n} y_{i}^{\ell} \in \mathbb{C}[\mathfrak{h}]^{W} \quad, \quad \ell \geq 0 .
$$

Let $\mathrm{H}_{\infty}=\mathrm{H}_{0, c}\left(S_{n}\right), c \neq 0$, be the rational Cherednik algebra of type $\mathbf{A}_{\mathbf{n}-\mathbf{1}}$, see (4.1). We may view $\psi_{\ell}, \phi_{\ell}$ as elements of $\mathbf{Z}_{\infty}$, the center of $\mathbf{H}_{\infty}$. Then, an easy calculation yields:

$$
\theta_{\psi_{k}}\left(\phi_{l}\right)=-k \cdot \sum_{p+q=l-1} \sum_{i=1}^{n} y_{i}^{p} x_{i}^{k-1} y_{i}^{q} .
$$

Using skew-symmetry of the Poisson bracket on $Z_{\infty}$, we obtain, as a corollary, the following a priori non-obvious result

Corollary 5.7 The element $\left\{\psi_{k}, \phi_{l}\right\} \in \mathrm{Z}_{\infty}$ is given by either of the following two expressions:

$$
\left\{\phi_{l}, \psi_{k}\right\}=k \cdot \sum_{p+q=l-1} \sum_{i=1}^{n} y_{i}^{p} x_{i}^{k-1} y_{i}^{q}=l \cdot \sum_{p+q=k-1} \sum_{i=1}^{n} x_{i}^{p} y_{i}^{l-1} x_{i}^{q} .
$$

We do not know how to check Corollary 5.7 by a direct computation.

Let $\mathbb{C}\langle x, y\rangle$ be the free associative algebra on two generators. Let $F$ be either an automorphism or a derivation of the algebra $\mathbb{C}\langle x, y\rangle$. It is clear that $F$ is uniquely determined by two non-commutative polynomials: $F(x)=: P_{F}(x, y)$ and $F(y)=: Q_{F}(x, y)$. In particular, for each integer $\ell \geq 0$, we define two one-parameter groups of automorphisms $a_{\tau, \ell}, b_{\tau, \ell},(\tau \in \mathbb{C})$, resp. two derivations $\alpha_{\ell}, \beta_{\ell}$, of the algebra $\mathbb{C}\langle x, y\rangle$ by the following assignments:

$$
\begin{aligned}
& a_{\tau, \ell}(x)=x+\tau y^{\ell}, a_{\tau, \ell}(y)=y, \text { and } b_{\tau, \ell}(x)=x, b_{\tau, \ell}(y)=y+\tau x^{\ell}, \text { resp. } \\
& \alpha_{\ell}(x)=y^{\ell}, \quad \alpha_{\ell}(y)=0, \quad \text { and } \beta_{\ell}(x)=0, \beta_{\ell}(y)=x^{\ell}, \quad \ell=0,1, \ldots .
\end{aligned}
$$

Definition 5.9 Let $\mathbb{G}$ denote the group of the automorphisms of $\mathbb{C}\langle x, y\rangle$ generated by the set $\left\{a_{\tau, \ell}, b_{\tau, \ell}\right\}_{\ell \geq 0}$, and let $\mathbb{L}$ denote the Lie algebra of all derivations of $\mathbb{C}\langle x, y\rangle$ generated by the set $\left\{\alpha_{\ell}, \beta_{\ell}\right\}_{\ell \geq 0}$.

Remark. Write $\operatorname{Aut}_{\omega} \mathbb{C}\langle x, y\rangle$, resp. $\operatorname{Der}_{\omega} \mathbb{C}\langle x, y\rangle$, for the group of all automorphisms $F: \mathbb{C}\langle x, y\rangle \rightarrow \mathbb{C}\langle x, y\rangle$ such that $F(x y-y x)=x y-y x$, resp. for the Lie algebra of all derivations $f: \mathbb{C}\langle x, y\rangle \rightarrow \mathbb{C}\langle x, y\rangle$ such that $f(x y-y x)=0$. It is clear that $\mathbb{G} \subset \operatorname{Aut}_{\omega} \mathbb{C}\langle x, y\rangle$, and it is known, see $\mathbb{M U}$, that in effect $\mathbb{G}=$ Aut $_{\omega} \mathbb{C}\langle x, y\rangle$. Similarly, one has: $\mathbb{L} \subset \operatorname{Der}_{\omega} \mathbb{C}\langle x, y\rangle$. Yet, this latter inclusion is not an equality, cf. Question 17.10. $\diamond$

Write $\operatorname{Aut}_{W}\left(\mathrm{H}_{\kappa}\right)$ for the group of automorphisms $a: \mathrm{H}_{\kappa} \rightarrow \mathrm{H}_{\kappa}$ that restrict to the identity on $\mathbb{C W} \subset \mathrm{H}_{\kappa}$. Similarly, let $\operatorname{Der}_{W}\left(\mathrm{H}_{\kappa}\right)$ denote the Lie algebra of all derivations $\gamma: \mathrm{H}_{\kappa} \rightarrow \mathrm{H}_{\kappa}$ such that $\gamma(\mathbb{C} W)=0$. 
Theorem 5.10 Let $\kappa \in \mathbb{P}^{1}$ and $\mathrm{H}_{\kappa}=\mathrm{H}_{\kappa}\left(S_{n}\right)$. For any $F \in \mathbb{G}$, resp. $F \in \mathbb{L}$, the assignment:

$$
x_{i} \mapsto P_{F}\left(x_{i}, y_{i}\right) \quad \text { and } \quad y_{i} \mapsto Q_{F}\left(x_{i}, y_{i}\right) \quad, \quad i=1, \ldots, n,
$$

extends uniquely to an algebra automorphism $a_{F}: \mathrm{H}_{\kappa} \rightarrow \mathrm{H}_{\kappa}$, resp. a derivation $\gamma_{F}$ : $\mathrm{H}_{\kappa} \rightarrow \mathrm{H}_{\kappa}$. The map: $F \mapsto a_{F}$, resp. $F \mapsto \gamma_{F}$, thus defined gives a group homomorphism: $\mathbb{G} \rightarrow \operatorname{Aut}_{W}\left(\mathrm{H}_{\kappa}\right)$, resp., a Lie algebra homomorphism: $\mathbb{L} \rightarrow \operatorname{Der}_{W}\left(\mathrm{H}_{\kappa}\right)$.

Proof. The proofs of the 'group' and 'Lie algebra' parts are completely analogous, so we prove only the Lie algebra part.

Formula (5.4) applied to the element $f=\psi_{\ell+1}$, resp. $f=\phi_{\ell+1}$, see (5.6), yields readily

$$
\gamma_{\psi_{\ell+1}}\left(y_{i}\right)=-(\ell+1) \cdot x_{i}^{\ell} \quad \text { and } \quad \gamma_{\phi_{\ell+1}}\left(x_{i}\right)=0 \quad, \quad \forall i=1, \ldots, n .
$$

It follows that, for $F=\alpha_{\ell} \in \mathbb{L}$, the assignment of the Theorem gives indeed a well-defined derivation $\gamma_{F} \in \operatorname{Der}_{W}\left(\mathrm{H}_{\kappa}\right)$. Similarly, applying formula (5.4) to $f=-\phi_{\ell+1}$ shows that, for $F=\beta_{\ell} \in \mathbb{L}$, the assignment of the Theorem gives indeed a well-defined derivation $\gamma_{F} \in \operatorname{Der}_{W}\left(\mathrm{H}_{\kappa}\right)$.

It is clear now that, if $F_{1}, F_{2} \in \mathbb{L}$ are such that the assignments $\gamma_{F_{1}}$ and $\gamma_{F_{2}}$ of the Theorem extend to derivations of $\mathrm{H}_{\kappa}$, then $\left[\gamma_{F_{1}}, \gamma_{F_{2}}\right]$ is a derivation of $\mathrm{H}_{\kappa}$ again, and one has:

$$
\left[\gamma_{F_{1}}, \gamma_{F_{2}}\right]: \quad x_{i} \mapsto P_{\left[F_{1}, F_{2}\right]}\left(x_{i}, y_{i}\right) \quad \text { and } \quad y_{i} \mapsto Q_{\left[F_{1}, F_{2}\right]}\left(x_{i}, y_{i}\right) \quad, \quad i=1, \ldots, n
$$

Thus, we get: $\left[\gamma_{F_{1}}, \gamma_{F_{2}}\right]=\gamma_{\left[F_{1}, F_{2}\right]}$. This completes the proof. 


\section{PART 2. HARISH-CHANDRA HOMOMORPHISMS}

\section{The radial part construction}

We begin with the following generalization of the classical construction of 'radial part' of an invariant differential operator.

Given a smooth complex algebraic variety $X$, let $\mathcal{D}(X)$ denote the algebra of regular differential operators on $X$. Further, given a (possibly infinite dimensional) $\mathbb{C}$-vector space $A$, we write $\mathcal{D}(X, A):=A \otimes \mathcal{D}(X)$ for the vector space of $A$-valued regular differential operators. If $A$ is an associative $\mathbb{C}$-algebra, the space $\mathcal{D}(X, A)$ acquires a natural associative algebra structure, the tensor product of that on $A$ and on $\mathcal{D}(X)$.

Let $G$ be a connected reductive algebraic group over $\mathbb{C}$ with Lie algebra $\mathfrak{g}$, and $T \subset G$ a maximal torus. Let $\mathfrak{g}^{\text {rs }} \subset \mathfrak{g}$ be the subset of semisimple regular elements. Let $\mathfrak{h} \subset \mathfrak{g}$ be the Cartan subalgebra corresponding to $T$, and write $\mathfrak{h}^{\text {reg }}=\mathfrak{h} \cap \mathfrak{g}^{\text {rs }}$ for the Zariski open subset formed by the regular elements of $\mathfrak{h}$, i.e. for the complement of the root hyperplanes. Let $\mathcal{U} \mathfrak{g}$ denote the enveloping algebra of the Lie algebra $\mathfrak{g}$, and $(\mathcal{U} \mathfrak{g})^{\text {ad } \mathfrak{h}}$ denote the centralizer of $\mathfrak{h}$ in $\mathcal{U} \mathfrak{g}$. Then $(\mathcal{U} \mathfrak{g})^{\text {ad } \mathfrak{h}} \cdot \mathfrak{h}$ is a two-sided ideal in the algebra $(\mathcal{U} \mathfrak{g})^{\text {ad } \mathfrak{h}}$, and we set $(\mathcal{U} \mathfrak{g})_{\mathfrak{h}}=(\mathcal{U} \mathfrak{g})^{\text {ad } \mathfrak{h}} /(\mathcal{U} \mathfrak{g})^{\text {ad } \mathfrak{h}} \cdot \mathfrak{h}$. The action of the Weyl group $W$ on $\mathfrak{h}$ preserves the subset $\mathfrak{h}^{\text {reg }}$, hence induces a $W$-action on $\mathcal{D}\left(\mathfrak{h}^{\text {reg }}\right)$ and on $(\mathcal{U} \mathfrak{g})_{\mathfrak{h}}$. Let $\mathcal{D}\left(\mathfrak{h}^{\text {reg }},(\mathcal{U} \mathfrak{g})_{\mathfrak{h}}\right)^{W}$ denote the subalgebra of $W$-invariants in the algebra $\mathcal{D}\left(\mathfrak{h}^{\text {reg }},(\mathcal{U} \mathfrak{g})_{\mathfrak{h}}\right)=(\mathcal{U} \mathfrak{g})_{\mathfrak{h}} \bigotimes_{\mathbb{C}} \mathcal{D}\left(\mathfrak{h}^{\text {reg }}\right)$.

Given a $\mathfrak{g}$-module $V$, let $V\langle 0\rangle:=V^{\mathfrak{h}}$ denote its zero weight subspace. It is clear that $V\langle 0\rangle$ is an $(\mathcal{U} \mathfrak{g})^{\text {ad } \mathfrak{h}}$-stable subspace, and the action of $(\mathcal{U} \mathfrak{g})^{\text {ad } \mathfrak{h}}$ on $V\langle 0\rangle$ factors through $(\mathcal{U} \mathfrak{g})_{\mathfrak{h}}$. Thus, the space $V\langle 0\rangle$ has a natural $(\mathcal{U} \mathfrak{g})_{\mathfrak{h}}$-module structure, and the space $V\langle 0\rangle \otimes$ $\mathbb{C}\left[\mathfrak{h}^{\text {reg }}\right]$ has a natural $\mathcal{D}\left(\mathfrak{h}^{\text {reg }},(\mathcal{U} \mathfrak{g})_{\mathfrak{h}}\right)$-module structure.

Proposition 6.1 There exists a canonical algebra isomorphism

$$
\widetilde{\Psi}: \mathcal{D}\left(\mathfrak{g}^{\text {rs }}\right)^{G} \stackrel{\sim}{\longrightarrow} \mathcal{D}\left(\mathfrak{h}^{\text {reg }},(\mathcal{U} \mathfrak{g})_{\mathfrak{h}}\right)^{W},
$$

such that, for any $f \in\left(V \otimes \mathbb{C}\left[\mathfrak{g}^{\text {rs }}\right]\right)^{\mathfrak{g}}$ and $L \in \mathcal{D}\left(\mathfrak{g}^{\text {rs }}\right)^{\mathfrak{g}}$, one has: $\left.(L f)\right|_{\mathfrak{h}^{\text {reg }}}=\widetilde{\Psi}(L)\left(\left.f\right|_{\mathfrak{h}^{\text {reg }}}\right)$.

Proof. The map $p: G / T \times \mathfrak{h}^{\text {reg }} \stackrel{W}{\longrightarrow} \mathfrak{g}^{\text {rs }},(g, h) \mapsto \operatorname{Ad} g(h)$, gives the standard Galois covering of $\mathfrak{g}^{\text {rs }}$ with the Galois group $W$, the Weyl group, see e.g. [CG]. By the properties of Galois covering, the pull-back via $p$ identifies the algebra $\mathcal{D}\left(\mathfrak{g}^{\text {rs }}\right)$ with $\mathcal{D}\left(G / T \times \mathfrak{h}^{\text {reg }}\right)^{W}$, the algebra of $W$-invariant differential operators on $G / T \times \mathfrak{h}^{\text {reg }}$. Now, the group $G$ acts on $G / T \times \mathfrak{h}^{\text {reg }}$ by means of left translations on the first factor $G / T$. The map $p$ intertwines this $G$-action with the Ad $G$-action on $\mathfrak{g}^{\text {rs }}$. It follows that pull-back via $p$ induces an algebra isomorphism: $\mathcal{D}\left(\mathfrak{g}^{\text {rs }}\right)^{G} \stackrel{\sim}{\sim} \mathcal{D}\left(G / T \times \mathfrak{h}^{\text {reg }}\right)^{G \times W}$ (note that the actions of $G$ and $W$ on $G / T \times \mathfrak{h}^{\text {reg }}$ commute).

In general, one has: $\mathcal{D}\left(G / T \times \mathfrak{h}^{\text {reg }}\right)=\mathcal{D}(G / T) \otimes \mathcal{D}\left(\mathfrak{h}^{\text {reg }}\right)$, hence, $\mathcal{D}\left(G / T \times \mathfrak{h}^{\text {reg }}\right)^{G}=$ $\mathcal{D}(G / T)^{G} \otimes \mathcal{D}\left(\mathfrak{h}^{\text {reg }}\right)$, since the group $G$ acts trivially on $\mathfrak{h}^{\text {reg }}$. We now recall the well-known isomorphism: $\mathcal{D}(G / T)^{G} \simeq(\mathcal{U} \mathfrak{g})_{\mathfrak{h}}$, see e.g. [He]. Thus, we obtain:

$$
\mathcal{D}\left(\mathfrak{g}^{\text {rs }}\right)^{G} \stackrel{\sim}{\longrightarrow} \mathcal{D}\left(G / T \times \mathfrak{h}^{\text {reg }}\right)^{G \times W} \simeq\left((\mathcal{U} \mathfrak{g})_{\mathfrak{h}} \otimes \mathcal{D}\left(\mathfrak{h}^{\text {reg }}\right)\right)^{W}=\mathcal{D}\left(\mathfrak{h}^{\text {reg }},(\mathcal{U} \mathfrak{g})_{\mathfrak{h}}\right)^{W} .
$$


The 'universal' Harish-Chandra homomorphism. Following the classical construction of Harish-Chandra, we will use not the radial part homomorphism $\widetilde{\Psi}$ but its modification, obtained by conjugating $\widetilde{\Psi}$ by the Weyl denominator. Namely, choose the set $R_{+} \subset R$ of positive roots, and let $\delta=\prod_{\alpha \in R_{+}} \alpha$ denote the product of positive roots. For any $L \in \mathcal{D}\left(\mathfrak{g}^{\text {rs }}\right)^{G}$, put $\Psi(L):=\delta \circ \widetilde{\Psi}(L) \circ \delta^{-1} \in \mathcal{D}\left(\mathfrak{h}^{\text {reg }},(\mathcal{U} \mathfrak{g})_{\mathfrak{h}}\right)^{W}$, that is, for any $f \in \mathbb{C}\left[\mathfrak{h}^{\text {reg }},(\mathcal{U} \mathfrak{g})_{\mathfrak{h}}\right]$, we have $\Psi(L): f \mapsto \delta^{-1} \cdot \widetilde{\Psi}(L)(\delta \cdot f)$. This definition does not depend on the choice of the set of positive roots.

Let $\left\{e_{\alpha}\right\}_{\alpha \in R}$, denote root vectors from a Weyl basis of the Lie algebra $\mathfrak{g}$, normalized so that $\left(e_{\alpha}, e_{-\alpha}\right)=1$.

Proposition 6.2 The image of the Laplacian $\Delta_{\mathfrak{g}} \in \mathcal{D}(\mathfrak{g})^{G}$ under the universal HarishChandra homomorphism $\Psi$ is given by the formula:

$$
\Psi\left(\Delta_{\mathfrak{g}}\right)=\Delta_{\mathfrak{h}}-\sum_{\alpha \in R} \frac{e_{\alpha} \cdot e_{-\alpha}}{\alpha^{2}} \quad \text { (Spin-Calogero-Moser operator) } .
$$

Proof. For $x \in \mathfrak{g}$, write $\frac{\partial}{\partial x}$ for the operator of directional derivative along $x$, abusing the notation put $e^{x}=\exp (x) \in G$. We have: $\Delta_{\mathfrak{g}}=\Delta_{\mathfrak{h}}+\sum_{\alpha} \frac{\partial^{2}}{\partial e_{-\alpha} \cdot \partial e_{\alpha}}$.

Let $V$ be a finite dimensional $\mathfrak{g}$-module, and $f$ a $\mathfrak{g}$-equivariant $V$-valued regular function on a neighborhood of $\mathfrak{h}^{\text {reg }}$ in $\mathfrak{g}^{\text {rs }}$. Fix $h \in \mathfrak{h}^{\text {reg }}$, and $s \in \mathbb{C}$. For $t \in \mathbb{C}$, we have

$e^{t \cdot e_{\alpha}}\left(f\left(h+s \cdot e_{-\alpha}\right)\right)=f\left(\operatorname{Ad}\left(e^{t \cdot e_{\alpha}}\right)\left(h+s \cdot e_{-\alpha}\right)\right)=f\left(h+s \cdot e_{-\alpha}-t \alpha(h) \cdot e_{\alpha}+s t \cdot h_{\alpha}+O\left(t^{2}\right)\right)$.

Differentiating this equation with respect to $t$, at $t=0$ we find

$$
e_{\alpha}\left(f\left(h+s \cdot e_{-\alpha}\right)\right)=-\alpha(h) \cdot \frac{\partial f}{\partial e_{\alpha}}\left(h+s \cdot e_{-\alpha}\right)+s \cdot \frac{\partial f}{\partial h_{\alpha}}\left(h+s \cdot e_{-\alpha}\right) .
$$

We rewrite the latter equation as follows

$$
\frac{\partial f}{\partial e_{\alpha}}\left(h+s \cdot e_{-\alpha}\right)=\frac{s}{\alpha(h)} \cdot \frac{\partial f}{\partial h_{\alpha}}\left(h+s \cdot e_{-\alpha}\right)-\frac{1}{\alpha(h)} \cdot e_{\alpha}\left(f\left(h+s \cdot e_{-\alpha}\right)\right) .
$$

Differentiating equation (6.4) with respect to $s$ at $s=0$, and using equation (6.4) for the root $-\alpha$, we obtain

$$
\frac{\partial^{2} f}{\partial e_{-\alpha} \partial e_{\alpha}}=\frac{1}{\alpha} \cdot \frac{\partial f}{\partial h_{\alpha}}-\frac{1}{\alpha^{2}} \cdot e_{\alpha} e_{-\alpha} f
$$

Thus, we find that

$$
\widetilde{\Psi}\left(\Delta_{\mathfrak{g}}\right)=\Delta_{\mathfrak{h}}+\sum_{\alpha \in R} \frac{1}{\alpha} \cdot \frac{\partial}{\partial h_{\alpha}}-\sum_{\alpha \in R} \frac{e_{\alpha} \cdot e_{-\alpha}}{\alpha^{2}}
$$

Conjugating this equation by $\delta$ kills the first order term in (6.5), and we are done. 
Now fix a $\mathcal{U} \mathfrak{g}$-module $V$. As has been explained above, the zero-weight space $V\langle 0\rangle=V^{\mathfrak{h}}$ is $(\mathcal{U} \mathfrak{g})^{\text {ad } \mathfrak{h}}$-stable, and is in effect an $(\mathcal{U} \mathfrak{g})_{\mathfrak{h}}=(\mathcal{U} \mathfrak{g})^{\text {ad } \mathfrak{h}} /(\mathcal{U} \mathfrak{g})^{\text {ad } \mathfrak{h}} \cdot \mathfrak{h}$-module. This way one gets an algebra homomorphism $\chi:(\mathcal{U} \mathfrak{g})_{\mathfrak{h}} \rightarrow \operatorname{End}_{\mathbb{C}} V\langle 0\rangle$. The composite homomorphism:

$$
\Psi_{V}: \quad \mathcal{D}(\mathfrak{g})^{\mathfrak{g}} \stackrel{\Psi}{\longrightarrow} \mathcal{D}\left(\mathfrak{h}^{\text {reg }},(\mathcal{U} \mathfrak{g})_{\mathfrak{h}}\right)^{W} \stackrel{\chi}{\longrightarrow} \mathcal{D}\left(\mathfrak{h}^{\text {reg }}, \operatorname{End}_{\mathbb{C}} V\langle 0\rangle\right)^{W}
$$

will be referred to as the Harish-Chandra homomorphism associated to $V$.

In the case of a finite-dimensional representation $V$, the construction of the homomorphism $\Psi_{V}$ can be equivalently described as follows. We start with the natural $\mathcal{D}(\mathfrak{g})^{\mathfrak{g}_{-}}$

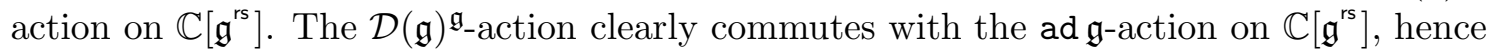
preserves each ad $\mathfrak{g}$-isotypic component of $\mathbb{C}\left[\mathfrak{g}^{\text {rs }}\right]$. This gives, for any finite-dimensional $\mathfrak{g}$-module $V$, a $\mathcal{D}(\mathfrak{g})^{\mathfrak{g}}$-module structure on the vector space $\operatorname{Hom}_{\mathfrak{g}}\left(V^{*}, \mathbb{C}\left[\mathfrak{g}^{\text {rs }}\right]\right)$. The space $\mathbb{C}\left[\mathfrak{g}^{\text {rs }}\right]$ has, by Frobenius reciprocity, see Kostant [Ko1], the following decomposition into ad $\mathfrak{g}$-isotypic components:

$$
\mathbb{C}\left[\mathfrak{g}^{\mathrm{rs}}\right]=\bigoplus_{\text {finite-dimensional simple } \mathfrak{g} \text {-modules } V} V^{*} \otimes V\langle 0\rangle \otimes \mathbb{C}\left[\mathfrak{g}^{\mathrm{rs}}\right]^{\mathfrak{g}}
$$

Therefore, we find:

$$
\operatorname{Hom}_{\mathfrak{g}}\left(V^{*}, \mathbb{C}\left[\mathfrak{g}^{\mathrm{rs}}\right]\right) \simeq V\langle 0\rangle \otimes \mathbb{C}\left[\mathfrak{g}^{\mathrm{rs}}\right]^{\mathfrak{g}} \simeq V\langle 0\rangle \otimes \mathbb{C}\left[\mathfrak{h}^{\text {reg }}\right]^{W}
$$

Thus, one obtains a $\mathcal{D}(\mathfrak{g})^{\mathfrak{g}}$-action on $V\langle 0\rangle \otimes \mathbb{C}\left[\mathfrak{h}^{\text {reg }}\right]^{W}$. It is easy to see that, for any $L \in$ $\mathcal{D}(\mathfrak{g})^{\mathfrak{g}}$, the action of $L$ arising in this way is given by a certain $\operatorname{End}_{\mathbb{C}} V\langle 0\rangle$-valued differential operator on $\operatorname{Spec}\left(\mathbb{C}\left[\mathfrak{h}^{\text {reg }}\right]^{W}\right)=\mathfrak{h}^{\text {reg }} / W$. But since the projection $\pi: \mathfrak{h}^{\text {reg }} \rightarrow \mathfrak{h}^{\text {reg }} / W$ is étale, the pull-back via $\pi$ gives a differential operator $\tilde{\Psi}_{V}(L) \in \mathcal{D}\left(\mathfrak{h}^{\text {reg }}, \operatorname{End}_{\mathbb{C}} V\langle 0\rangle\right)$. Then one can define $\Psi_{V}(L)$ by $\Psi_{V}(L)=\delta \circ \tilde{\Psi}_{V}(L) \circ \delta^{-1}$. We leave it to the reader to check that the map: $L \mapsto \Psi_{V}(L)$ thus defined is nothing but the one given by formula (6.6).

'The' Harish-Chandra homomorphism. Let $\Phi: \mathcal{D}(\mathfrak{g})^{\mathfrak{g}} \rightarrow \mathcal{D}\left(\mathfrak{h}^{\text {reg }}\right)^{W}$ denote the Harish-Chandra homomorphism associated to the trivial 1-dimensional $\mathfrak{g}$-module $\mathbb{C}$. It is easy to see that the following definition agrees with the classical notion of the HarishChandra homomorphism:

Definition 6.7 The map $\Phi$ is called 'the' Harish-Chandra homomorphism.

By a fundamental result of Harish-Chandra, [HC], the image of $\Phi$ consists of regular differential operators on the whole of $\mathfrak{h}$. This is not true for Harish-Chandra homomorphisms associated to nontrivial $\mathfrak{g}$-modules $V$.

Recently, thanks to Wallach, and Levasseur-Stafford, the results of Harish-Chandra have been strengthened as follows. Let ad : $\mathfrak{g} \hookrightarrow \mathcal{D}(\mathfrak{g})$ denote the Lie algebra map sending $x \in \mathfrak{g}$ to the vector field ad $x$ on $\mathfrak{g}$. Form the left ideal $\mathcal{D}(\mathfrak{g}) \cdot \operatorname{ad} \mathfrak{g} \subset \mathcal{D}(\mathfrak{g})$, generated by the image of the map ad, and set $I_{\text {ad }}:=(\mathcal{D}(\mathfrak{g}) \cdot$ ad $\mathfrak{g}) \cap \mathcal{D}(\mathfrak{g})^{\mathfrak{g}}$. It is clear that $I_{\text {ad }}$ is a two-sided ideal in $\mathcal{D}(\mathfrak{g})^{\mathfrak{g}}$; moreover, the Harish-Chandra homomomorphism $\Phi$ vanishes on $I_{\text {ad }}$, hence descends to a map $\Phi: \mathcal{D}(\mathfrak{g})^{\mathfrak{g}} / I_{\text {ad }} \rightarrow \mathcal{D}(\mathfrak{h})^{W}$. The theorem below summarizes the results of Wallach [Wa], and Levasseur-Stafford [LS1, LS2]. 
Theorem 6.8 The map $\Phi: \mathcal{D}(\mathfrak{g})^{\mathfrak{g}} / I_{\mathrm{ad}} \stackrel{\sim}{\longrightarrow} \mathcal{D}(\mathfrak{h})^{W}$ is an algebra isomorphism.

The algebras $\mathcal{D}(\mathfrak{g})^{\mathfrak{g}}$ and $\mathcal{D}(\mathfrak{h})^{W}$ come equipped with canonical filtrations by the order of differential operators, and there are canonical algebra isomorphisms: $\operatorname{gr}\left(\mathcal{D}(\mathfrak{g})^{\mathfrak{g}}\right) \simeq$ $\mathbb{C}[\mathfrak{g} \oplus \mathfrak{g}]^{\mathfrak{g}}$, and $\operatorname{gr}\left(\mathcal{D}(\mathfrak{h})^{W}\right) \simeq \mathbb{C}[\mathfrak{h} \oplus \mathfrak{h}]^{W}$. In particular, $\operatorname{gr}\left(I_{\mathrm{ad}}\right) \subset \operatorname{gr}\left(\mathcal{D}(\mathfrak{g})^{\mathfrak{g}}\right)$ may be viewed as an ideal in $\mathbb{C}[\mathfrak{g} \oplus \mathfrak{g}]^{\mathfrak{g}}$. One can describe this ideal as follows.

Let $J \subset \mathbb{C}[\mathfrak{g} \oplus \mathfrak{g}]$ denote the ideal generated by the functions: $\mathfrak{g} \oplus \mathfrak{g} \rightarrow \mathbb{C}$ of the form: $(X, Y) \mapsto \lambda([X, Y]), \lambda \in \mathfrak{g}^{*}$. Write $\sqrt{J}$ for the radical of $J$, and let: $(\sqrt{J})^{\mathfrak{g}}=$ $\sqrt{J} \cap \mathbb{C}[\mathfrak{g} \oplus \mathfrak{g}]^{\mathfrak{g}}$.

Theorem $6.9([\overline{\mathbf{L}}])$ One has: $\operatorname{gr}\left(I_{\mathrm{ad}}\right)=(\sqrt{J})^{\mathfrak{g}}$.

This theorem has the following geometric meaning. Let $\mathcal{Z}=\{(X, Y) \in \mathfrak{g} \oplus \mathfrak{g} \mid[X, Y]=$ $0\}$ be the commuting variety of $\mathfrak{g}$. By Hilbert Nullstellensatz, $\mathbb{C}[\mathcal{Z}]=\mathbb{C}[\mathfrak{g} \oplus \mathfrak{g}] / \sqrt{J}$. Theorem 6.9 says: $\operatorname{gr}\left(\mathcal{D}(\mathfrak{g})^{\mathfrak{g}}\right) / \operatorname{gr}\left(I_{\text {ad }}\right)=\mathbb{C}[\mathcal{Z}]$. Observe now that the natural inclusion $i: \mathfrak{h} \oplus \mathfrak{h} \hookrightarrow \mathcal{Z}$ gives the restriction map $i^{*}: \mathbb{C}[\mathcal{Z}] \longrightarrow \mathbb{C}[\mathfrak{h} \oplus \mathfrak{h}]$.

Theorem 6.10 (i) The map $i^{*}$ induces an algebra isomorphism: $\mathbb{C}[\mathcal{Z}]^{G} \stackrel{\sim}{\longrightarrow} \mathbb{C}[\mathfrak{h} \oplus \mathfrak{h}]^{W}$.

(ii) The associated graded map $\operatorname{gr}(\Phi): \operatorname{gr}\left(\mathcal{D}(\mathfrak{g})^{\mathfrak{g}}\right) / \operatorname{gr}\left(I_{\text {ad }}\right) \longrightarrow \operatorname{gr}\left(\mathcal{D}(\mathfrak{h})^{W}\right)$ coincides with the map in (i), hence is an isomorphism.

The map $i^{*}: \mathbb{C}[\mathcal{Z}]^{G} \rightarrow \mathbb{C}[\mathfrak{h} \oplus \mathfrak{h}]^{W}$ is a double-analog of the Chevalley restriction isomorphism. Injectivity of $i^{*}$ is easy for $\mathfrak{g}=\mathfrak{g l}_{n}$, and is due to Richardson, in general. Surjectivity of $i^{*}$ follows, for $\mathfrak{g}=\mathfrak{g l}_{n}$, from classical invariant theory [We], and is due to Joseph $[\mathrm{J}]$ in the general case.

Remark. The ideal $I_{\text {ad }} \subset \mathcal{D}(\mathfrak{g})^{\mathfrak{g}}$ can be thought of as a non-commutative analogue of the ideal $J^{\mathfrak{g}} \subset \mathbb{C}[\mathfrak{g} \oplus \mathfrak{g}]^{\mathfrak{g}}$. In view of Theorem 6.9 we can regard Theorem 6.8, saying that $I_{\mathrm{ad}}=\operatorname{Ker}(\Phi)$, as a non-commutative analog of the equality $J^{\mathfrak{g}}=(\sqrt{J})^{\mathfrak{g}}$. We cannot prove this equality; it is a special case of a well known conjecture saying that $J=\sqrt{J}$.

\section{Deformation of the Harish-Chandra homomorphism}

We would like to construct a 1-parameter deformation of the Harish-Chandra homomorphism. To do this, we construct a 1-parameter family of representations $\left\{V_{k}\right\}_{k \in \mathbb{C}}$ of $\mathfrak{g}$, such that, for any $k$, the space $V_{k}\langle 0\rangle$ is 1-dimensional, and $V_{0}\langle 0\rangle$ is the trivial representation of $(\mathcal{U} \mathfrak{g})_{\mathfrak{h}}$. Then we may define a deformation of $\Phi$ by setting $\Phi_{\kappa}=\Psi_{V_{k}}$.

We now produce a particular 1-parameter family of representations $V_{k}$ in the case $\mathfrak{g}=\mathfrak{g l}_{n}$ (it turns out that such a family does not exist for Lie algebras which are not of type A). We think of $\mathfrak{g l}_{n}$ as the Lie algebra of linear vector fields on the vector space $\mathbb{C}^{n}$, with coordinates $x_{1}, \ldots, x_{n}$, so that $E_{i j}=x_{i} \frac{\partial}{\partial x_{j}}$ are the matrix units forming the standard basis of $\mathfrak{g l}_{n}$. Define $V_{k}$ to be the $\mathbb{C}$-vector space formed by all the expressions $\left(x_{1} \cdot \ldots \cdot x_{n}\right)^{k} \cdot P$, where $P$ is a Laurent polynomial in $x_{1}, \ldots, x_{n}$ of total degree zero, 
with the Lie algebra $\mathfrak{g l}_{n}$ acting by formal differentiation. Specifically, for each $i, j$, there is a well-defined action on the expressions above of the differential operator $x_{i} \frac{\partial}{\partial x_{j}}$. The assignment: $E_{i j} \mapsto x_{i} \frac{\partial}{\partial x_{j}}$ makes $V_{k}$ an $\mathfrak{g l}_{n}$-module. The $\mathfrak{g l}_{n}$-module that we need is obtained from this one by redefining the action of the center of $\mathfrak{g l}_{n}$. Specifically, we first consider $V_{k}$ as an $\mathfrak{s l}_{n}$-module, obtained by restricting the action from $\mathfrak{g l}_{n}$ to $\mathfrak{s l}_{n}$. Now, define a new $\mathfrak{g l}_{n}$-action on $V_{k}$ by pulling-back the $\mathfrak{s l}_{n}$-action via the natural Lie algebra projection: $\mathfrak{g l}_{n} \rightarrow \mathfrak{s l}_{n}$, so that the center of $\mathfrak{g l}_{n}$ acts trivially. This is the $\mathfrak{g l}_{n}$-module $V_{k}$ that we wanted to produce. The representation $V_{k}$ has an interpretation in terms of twisted differential operators explained in Appendix B.

It is clear that the $\mathfrak{g}$-module $V_{k}$ thus obtained has a 1-dimensional zero weight space $V_{k}\langle 0\rangle$, spanned by the element $\left(x_{1} \cdot \ldots \cdot x_{n}\right)^{k} \cdot 1$, and $V_{0}\langle 0\rangle$ is the trivial representation of $(\mathcal{U} \mathfrak{g})_{\mathfrak{h}}$.

Definition 7.1 For $\mathfrak{g}=\mathfrak{g l}_{n}$, or $\mathfrak{g}=\mathfrak{s l}_{n}$, we call $\Phi_{k}=\Psi_{V_{k}}: \mathcal{D}(\mathfrak{g})^{\mathfrak{g}} \rightarrow \mathcal{D}\left(\mathfrak{h}^{\text {reg }}\right)^{W}$ the deformed Harish-Chandra homomorphism with parameter $k \in \mathbb{C}$.

It is clear that if $k=0$, then the map $\Phi_{k}$ coincides with $\Phi$ (this follows from the fact that any equivariant function on $\mathfrak{g}^{\text {rs }}$ with values in $V_{0}$ is, in effect, an invariant function taking values in the trivial 1-dimensional subrepresentation of $V_{0}$ ).

Let $\mathcal{C}_{k}$, denote the centralizer in $\widetilde{\mathcal{D}\left(\mathfrak{h}^{\text {reg }}\right)^{W}}$ of the Calogero-Moser differential operator $\mathrm{L}_{k}$, see (1.16). Identify $(S \mathfrak{g})^{\mathfrak{g}}$ with the algebra of ad $\mathfrak{g}$-invariant constant coefficient differential operators on $\mathfrak{g}$.

Proposition 7.2 (i) For any $k \in \mathbb{C}$, we have: $\Phi_{k}\left(\Delta_{\mathfrak{g}}\right)=\mathrm{L}_{k}$.

(ii) The map $\Phi_{k}$ gives an algebra isomorphism: $(S \mathfrak{g})^{\mathfrak{g}} \stackrel{\sim}{\longrightarrow} \mathcal{C}_{k}$.

Proof. For any root $\alpha$, one finds that: $\left.e_{\alpha} e_{-\alpha}\right|_{V_{k}\langle 0\rangle}=k(k+1) \cdot \operatorname{Id}_{V_{k}\langle 0\rangle}$. Part (i) now follows from Proposition 6.2. To prove (ii), observe first that any element of $\Phi_{k}\left((S \mathfrak{g})^{\mathfrak{g}}\right)$ commutes with $\Phi_{k}\left(\Delta_{\mathfrak{g}}\right)=\mathrm{L}_{k}$, since $(S \mathfrak{g})^{\mathfrak{g}}$ is a commutative algebra, and belongs to $\overline{\mathcal{D}\left(\mathfrak{h}^{\text {reg }}\right)^{W}}$. It follows that $\Phi_{k}\left((S \mathfrak{g})^{\mathfrak{g}}\right) \subset \mathcal{C}_{k}$. Next, equip $(S \mathfrak{g})^{\mathfrak{g}}$ and $\mathcal{C}_{k}$ with filtrations given by the order of differential operator. The map $\Phi_{k}$ is clearly filtration preserving, hence, induces a map $\operatorname{gr}\left(\Phi_{k}\right): \operatorname{gr}\left((S \mathfrak{g})^{\mathfrak{g}}\right) \rightarrow \operatorname{gr}\left(\mathcal{C}_{k}\right)$. We have an obvious isomorphism: $\operatorname{gr}\left((S \mathfrak{g})^{\mathfrak{g}}\right) \simeq(S \mathfrak{g})^{\mathfrak{g}}$. Further, according to Opdam [O], cf. also VSC, there exists an algebra isomorphism $\sigma_{k}: S \mathfrak{h}^{W} \stackrel{\sim}{\longrightarrow} \mathcal{C}_{k}$, such that, for any homogeneous $u \in S \mathfrak{h}^{W}$ of degree $\ell$, the the operator $\sigma_{k}(u)$ has order $\ell$, and its principal symbol equals $u$. It follows that $\operatorname{gr}\left(\mathcal{C}_{k}\right)=S \mathfrak{h}^{W}$. Therefore, we may identify the map $\operatorname{gr}\left(\Phi_{k}\right)$ with the Chevalley isomorphism: $(S \mathfrak{g})^{\mathfrak{g}} \stackrel{\sim}{\longrightarrow} S \mathfrak{h}^{W}$. Hence, $\operatorname{gr}\left(\Phi_{k}\right)$ is bijective. Thus, $\Phi_{k}$ is a bijection as well.

Remark. Part (i) also follows from [E, EFK. Specifically, in EFK the authors consider radial parts of differential operators on the group $G$, rather than on its Lie algebra, and obtain Calogero-Moser operators with trigonometric potential $k(k+1) / \sin ^{2}(x)$ rather than $k(k+1) / x^{2}$. The rational case may be obtained by taking an appropriate limit of the result of EFK. 
Now recall that we have defined in the introduction the algebra $\mathcal{B}_{k} \subset \mathcal{D}\left(\mathfrak{h}^{\text {reg }}\right)^{W}$ generated by $\mathcal{C}_{k}$ and by the polynomial algebra, $\mathbb{C}[\mathfrak{h}]^{W} \subset \mathcal{D}(\mathfrak{h})^{W}$, of zero order $W$-invariant operators on $\mathfrak{h}$.

Recall the Lie algebra map ad $: \mathfrak{g} \rightarrow \mathcal{D}(\mathfrak{g}), x \mapsto$ ad $x$. This Lie algebra map extends uniquely to an associative algebra homomorphism ad $: \mathcal{U} \mathfrak{g} \rightarrow \mathcal{D}(\mathfrak{g})$. Further, let $\operatorname{Ann} V_{k} \subset$ $\mathcal{U} \mathfrak{g}$ denote the annihilator of the representation $V_{k}$, or, equivalently, the kernel of (13.1). Thus, $A n n V_{k}$ is a two-sided ideal in $\mathcal{U} \mathfrak{g}$, and it is easy to see that ad $\mathfrak{g}$-invariants in the left ideal $\mathcal{D}(\mathfrak{g}) \cdot \operatorname{ad}\left(\operatorname{Ann} V_{k}\right) \subset \mathcal{D}(\mathfrak{g})$ form a two-sided ideal: $\left(\mathcal{D}(\mathfrak{g}) \cdot \operatorname{ad}\left(\operatorname{Ann} V_{k}\right)\right)^{\mathfrak{g}} \subset \mathcal{D}(\mathfrak{g})^{\mathfrak{g}}$.

The following theorem is one of the main results of Part 2 of this paper.

Theorem 7.3 (i) For any $k \in \mathbb{C}$, we have: $\mathcal{B}_{k}=\operatorname{Im}\left(\Phi_{k}\right)$.

(ii) $\operatorname{Ker}\left(\Phi_{k}\right)=\left(\mathcal{D}(\mathfrak{g}) \cdot \operatorname{ad}\left(\operatorname{Ann} V_{k}\right)\right)^{\mathfrak{g}}$, for all $k \in \mathbb{C}$, except possibly a finite set.

Remark. The definition of the homomorphism $\Phi_{k}$ easily implies that, for any $k \in \mathbb{C}$, one has the inclusion: $\left(\mathcal{D}(\mathfrak{g}) \cdot \operatorname{ad}\left(\operatorname{Ann} V_{k}\right)\right)^{\mathfrak{g}} \subset \operatorname{Ker}\left(\Phi_{k}\right)$, which is (for generic $k$ ) a part of claim (ii) of the Theorem. The opposite inclusion is harder, it will be proved only in $\S 10$. Further, notice that $\Phi_{k}\left(\mathbb{C}[\mathfrak{g}]^{\mathfrak{g}}\right)=\mathbb{C}[\mathfrak{h}]^{W}$. Hence, Proposition 7.2 yields an inclusion: $\mathcal{B}_{k} \subset \operatorname{Im}\left(\Phi_{k}\right)$, which is part of claim (i) of the Theorem. The opposite inclusion is more difficult and will be proved in $\S 10$.

Corollary 7.4 For almost all $k \in \mathbb{C}$, the map $\Phi_{k}$ induces an algebra isomorphism

$$
\Phi_{k}: \mathcal{D}(\mathfrak{g})^{\mathfrak{g}} /\left(\mathcal{D}(\mathfrak{g}) \cdot \operatorname{ad}\left(\operatorname{Ann} V_{k}\right)\right)^{\mathfrak{g}} \stackrel{\sim}{\longrightarrow} \mathcal{B}_{k} .
$$

Hamiltonian interpretation: The Ad $G$-action on $\mathfrak{g}=\mathfrak{s l}_{n}$ gives rise to a Hamiltonian $G$-action on $T^{*} \mathfrak{g}$, the cotangent bundle on $\mathfrak{g}$, with moment map $\mu: T^{*} \mathfrak{g} \rightarrow \mathfrak{g}^{*}$. The algebra homomorphism ad $: \mathcal{U} \mathfrak{g} \rightarrow \mathcal{D}(\mathfrak{g})$ may be viewed as a "quantization" of the pullback morphism $\mu^{*}: \mathbb{C}\left[\mathfrak{g}^{*}\right] \rightarrow \mathbb{C}\left[T^{*} \mathfrak{g}\right]$.

Further, it is known, see e.g. [BJ], that $\operatorname{Ann} V_{k}$ is the primitive ideal in $\mathcal{U} \mathfrak{g}$ associated to the 'minimal' coadjoint orbit $\mathrm{O}_{k}:=\left\{s \in \mathfrak{s l}_{n} \simeq \mathfrak{g}^{*} \mid \operatorname{rk}\left(s-\left(k+\frac{1}{2}\right) \cdot \mathrm{Id}\right)=1\right\}$, via the Geometric quantization construction. Put $\mathfrak{I}_{\text {quantum }}:=A \mathrm{Ann} V_{k}$, and let $\mathfrak{I}_{\text {classic }}$ denote the defining ideal of the (closed) orbit $\mathrm{O}_{k}$. Thus, according to the "geometric quantization" philosophy, the algebra $\mathcal{U} \mathfrak{g} / \mathfrak{I}_{\text {quantum }}$ should be thought of as a quantization of $\mathbb{C}\left[\mathrm{O}_{k}\right]=\mathbb{C}\left[\mathfrak{g}^{*}\right] / \mathfrak{I}_{\text {classic }}$.

As has been discovered by Kazhdan-Kostant-Sternberg [KKS], the classical CalogeroMoser space can be obtained as the Hamiltonian reduction: $\mu^{-1}\left(\mathrm{O}_{k}\right) / G$, cf. also $\S 11$ below. The coordinate ring of $\mu^{-1}\left(\mathrm{O}_{k}\right) / G$, an affine algebraic variety, by definition is: $\mathbb{C}\left[\mu^{-1}\left(\mathrm{O}_{k}\right) / G\right]=\mathbb{C}\left[T^{*} \mathfrak{g}\right]^{\mathfrak{g}} /\left(\mathbb{C}\left[T^{*} \mathfrak{g}\right] \cdot \mu^{*} \mathfrak{I}_{\text {classic }}\right)^{\mathfrak{g}}$. Thus, the non-commutative algebra $\mathcal{D}(\mathfrak{g})^{\mathfrak{g}} /\left(\mathcal{D}(\mathfrak{g}) \cdot \operatorname{ad}\left(\mathfrak{I}_{\text {quantum }}\right)\right)^{\mathfrak{g}}$ is a natural "quantization" of the coordinate ring of the classical Calogero-Moser space. According to Corollary 7.4, this is nothing but the image of the deformed Harish-Chandra homomorphism.

Spherical Harish-Chandra isomorphism. Let $\mathrm{H}_{t, c}$ be the rational Cherednik algebra associated to the root system $\mathbf{A}_{\mathbf{n}-\mathbf{1}}$. In this case the function $c: R \rightarrow \mathbb{C}$ reduces to a 
constant. As has been explained in the Introduction, we may (and will) view the parameter $\kappa=(t, c)$ as a point $\kappa=c / t \in \mathbb{C} \cup\{\infty\}$. The special case: $t=0$ corresponds to the point: $\kappa=\infty \in \mathbb{P}^{1}$.

Let $t \neq 0$. Then, inverting the bijection $\Theta_{\kappa}^{\text {spher }}$ of Theorem 4.8(i), see also Proposition 4.9, we obtain an algebra homomorphism, called the 'spherical Harish-Chandra homomorphism', cf., (1.20):

$$
\Phi_{\kappa}^{\text {spher }}=\left(\Theta_{\kappa}^{\text {spher }}\right)^{-1} \circ \Phi_{\kappa}: \quad \mathcal{D}\left(\mathfrak{g l}_{n}\right)^{\mathfrak{g l}_{n}} \longrightarrow \mathbf{e H}_{\kappa} \mathbf{e} \quad, \quad \forall \kappa \in \mathbb{P}^{1} \backslash\{\infty\} .
$$

By Corollary 7.4, the induced map $\Phi_{\kappa}^{\text {spher }}: \mathcal{D}(\mathfrak{g})^{\mathfrak{g}} /\left(\mathcal{D}(\mathfrak{g}) \cdot \operatorname{ad}\left(\operatorname{Ann} V_{k}\right)\right)^{\mathfrak{g}} \longrightarrow \mathbf{e H}_{\kappa} \mathbf{e}$ is an isomorphism for almost all $\kappa \in \mathbb{C}$, making $\mathbf{e H}_{\kappa} \mathbf{e}$ a quantum Hamiltonian reduction of the algebra $\mathcal{D}(\mathfrak{g})$.

Proposition 7.6 For $\mathfrak{g}=\mathfrak{g l}_{n}$, the spherical Harish-Chandra homomorphism, $\Phi_{\kappa}^{\text {spher }}$, intertwines the standard Fourier automorphism on $\mathcal{D}(\mathfrak{g})^{\mathfrak{g}}$ and the automorphism $\mathrm{F}$ on $\mathbf{e H}_{\kappa} \mathbf{e}$.

Proof. This is clear, since $\mathrm{F}$ permutes $\mathbb{C}[\mathfrak{h}]^{W}$ and $\mathcal{C}_{\kappa}$.

Let $x_{i j}, i, j=1, \ldots, n$, be the coordinates on $\mathfrak{g}=\mathfrak{g l}_{n}$ corresponding to the standard basis formed by the matrix units. Let $X=\left\|x_{i j}\right\|, Y=\left\|\frac{\partial}{\partial x_{j i}}\right\|$. For each $\ell=0,1,2, \ldots$, the assignments, cf. 5.8

$a_{\tau, \ell}(X)=X+\tau Y^{\ell}, a_{\tau, \ell}(Y)=Y$, resp. $, b_{\tau, \ell}(X)=X, b_{\tau, \ell}(Y)=Y+\tau X^{\ell}, \tau \in \mathbb{C}$,

give rise to well-defined algebra automorphisms $a_{\tau, \ell}, b_{\tau, \ell}: \mathcal{D}(\mathfrak{g}) \rightarrow \mathcal{D}(\mathfrak{g})$. This way, one defines an action of the group $\mathbb{G}$, see Definition 5.9 , on $\mathcal{D}(\mathfrak{g})$ by algebra automorphisms. Furthermore, it is straightforward to verify that the $\mathbb{G}$-action commutes with the ad $\mathfrak{g}$ action on $\mathcal{D}(\mathfrak{g})$. In particular, the subalgebra $\mathcal{D}(\mathfrak{g})^{\mathfrak{g}}$ is stable under the $\mathbb{G}$-action.

Similarly, one defines an action of the Lie algebra $\mathbb{L}$ on $\mathcal{D}(\mathfrak{g})^{\mathfrak{g}}$ by algebra derivations. The following result incorporates Proposition 7.6 as a very special case.

Proposition 7.7 For any $\kappa \in \mathbb{P}^{1} \backslash\{\infty\}$, the spherical Harish-Chandra homomorphism (7.5) intertwines the $\mathbb{G}$-actions, resp. the $\mathbb{L}$-actions, on $\mathcal{D}\left(\mathfrak{g l}_{n}\right)^{\mathfrak{g l}_{n}}$ and on $\mathbf{e} \mathrm{H}_{\kappa} \mathbf{e}$.

Proof. Let us prove the statement in the Lie algebra case (the group case is entirely analogous). By definition, for any $\ell$, the action of the derivation $\alpha_{\ell}$ on $\mathcal{D}\left(\mathfrak{g l}_{n}\right)$, resp. on $\mathrm{H}_{\kappa}$, is given by the formula:

$$
\alpha_{\ell}=\left.\frac{d}{d \tau}\right|_{\tau=0} \operatorname{Ad}\left(e^{-\tau \cdot \operatorname{Tr}\left(X^{\ell+1} /(\ell+1)\right)}\right) ; \quad \text { resp. } \quad \alpha_{\ell}=\left.\frac{d}{d \tau}\right|_{\tau=0} \operatorname{Ad}\left(e^{-\tau \sum_{i} x_{i}^{\ell+1} /(\ell+1)}\right) .
$$

Since $\Phi_{\kappa}^{\text {spher }}$ clearly takes the function: $X \mapsto \operatorname{Tr}\left(X^{\ell+1}\right)$, viewed as an element of $\mathcal{D}(\mathfrak{g})^{\mathfrak{g}}$, to $\sum_{i} x_{i}^{\ell+1} \in \mathrm{H}_{\kappa}$, it follows that $\Phi_{\kappa}^{\text {spher }}$ commutes with $\alpha_{\ell}, \forall \ell \geq 0$.

Further, we know that $\Phi_{\kappa}\left(\Delta_{\mathfrak{g}}\right)=\mathrm{L}_{\kappa}$, and $\Theta_{\kappa}^{\text {spher }}\left(\mathbf{e} \cdot \Delta_{\mathfrak{h}} \cdot \mathbf{e}\right)=\mathrm{L}_{\kappa}$, where $\mathrm{L}_{\kappa}$ is the CalogeroMoser operator, and $\Delta_{\mathfrak{h}}=\sum_{i} y_{i}^{2}$ denotes the element of $\mathrm{H}_{\kappa}$ corresponding to the quadratic 
Casimir, viewed as an element of $\mathbb{C}[\mathfrak{h}]^{W} \subset \mathrm{H}_{\kappa}$. Hence, $\Phi_{\kappa}^{\text {spher }}\left(\Delta_{\mathfrak{g}}\right)=\left(\Theta_{\kappa}^{\text {spher }}\right)^{-1} \circ \Phi_{\kappa}\left(\Delta_{\mathfrak{g}}\right)=$ $\left(\Theta_{\kappa}^{\text {spher }}\right)^{-1}\left(\mathrm{~L}_{\kappa}\right)=\mathbf{e} \cdot \Delta_{\mathfrak{h}} \cdot \mathbf{e}$.

It follows that the map $\Phi_{\kappa}^{\text {spher }}$ commutes with the derivation $\beta_{2}$. But the derivations $\left\{\alpha_{2}, \beta_{2}\right\}$ generate the Lie algebra $\mathfrak{s l}_{2}$ that corresponds to the group of standard $\mathrm{SL}_{2^{-}}$ automorphisms, see Corollary 5.3. Therefore, $\Phi_{\kappa}^{\text {spher }}$ commutes with the standard $\mathrm{SL}_{2^{-}}$ automorphisms, in particular, with the Fourier transform F. Thus, for any $\ell$, the map $\Phi_{\kappa}^{\text {spher }}$ commutes with $\beta_{\ell}=\mathrm{F} \circ \alpha_{\ell} \circ \mathrm{F}^{-1}$, and the Proposition follows.

\section{Example: $\mathfrak{g}=\mathfrak{s l}_{2}$}

In the case $\mathfrak{g}=\mathfrak{s l}_{2}$, we will explicitly compute the source and the target of the map $\Phi_{k}$ of Corollary 7.4 and verify directly that both are flat deformations and that this map is an isomorphism.

We first give an explicit description of the algebra $\mathcal{D}(\mathfrak{g})^{\mathfrak{g}} /\left(\mathcal{D}(\mathfrak{g}) \cdot \operatorname{ad}\left(\operatorname{Ann} V_{k}\right)\right)^{\mathfrak{g}}$ in terms of generators and relations. To this end, it is convenient to identify $\mathfrak{g}$ with the Lie algebra $\mathfrak{s o}_{3}$, so that the adjoint representation of $\mathfrak{g}$ becomes the tautological representation of $\mathfrak{s o}_{3}$ on $\mathbb{C}^{3}$. Thus, the algebra $\mathcal{D}(\mathfrak{g})^{\mathfrak{g}}$ gets identified with the algebra of rotation-invariant differential operators on $\mathbb{C}^{3}$. We have the following classical result (see e.g. Sch ).

Proposition 8.1 The algebra $\mathcal{D}\left(\mathbb{C}^{3}\right)^{\mathfrak{s o}_{3}}$ is generated by the squared radius $r^{2}$, the second order Laplacian $\Delta$, and the Euler field eu.

Proof. It is the standard result in elementary invariant theory that the commutative algebra $\mathbb{C}\left[\mathbb{C}^{3} \oplus \mathbb{C}^{3}\right]^{\mathfrak{s o}_{3}}$ is generated by 3 elements: $\mathbf{r}^{\mathbf{2}}, \mathbf{r p}, \mathbf{p}^{\mathbf{2}}$, where $\mathbf{r}$ is the radius vector, and $\mathbf{p}$ is the momentum. Now, equip $\mathcal{D}\left(\mathbb{C}^{3}\right)^{\mathfrak{s o}_{3}}$ with filtration by the order of differential operators. Then, we have: $\operatorname{gr}\left(\mathcal{D}\left(\mathbb{C}^{3}\right)^{\mathfrak{s o}_{3}}\right)=\mathbb{C}\left[\mathbb{C}^{3} \oplus \mathbb{C}^{3}\right]^{\mathfrak{s o}_{3}}$, and our claim follows from its analogue for $\mathbb{C}\left[\mathbb{C}^{3} \oplus \mathbb{C}^{3}\right]^{\mathfrak{s o}_{3}}$, mentioned above.

Let $e, h, f$ be the standard $\mathfrak{s l}_{2}$-triple, $\mathfrak{g}=\langle e, h, f\rangle$. Proposition 8.1 shows that $\mathcal{D}(\mathfrak{g})^{\mathfrak{g}}$ can be identified with $\mathcal{U} \mathfrak{g}$, via the map given by the assignment: $-\frac{1}{2} r^{2} \mapsto f, \frac{1}{2} \Delta \mapsto e$.

Next, we give an explicit description of the algebra $\mathcal{B}_{k}$ in terms of generators and relations. By the definition, $\mathcal{B}_{k}$ is generated by the operators $x^{2}$ and $\partial^{2}-\frac{k(k+1)}{x^{2}}$ in one variable $(\partial$ denotes the differentiation with respect to $x$ ).

Let $C=e f+f e+h^{2} / 2 \in \mathcal{U}\left(\mathfrak{s l}_{2}\right)$ be the quadratic Casimir, and set: $c_{k}=\frac{1}{2}\left(k-\frac{1}{2}\right)\left(k+\frac{3}{2}\right)$.

Proposition 8.2 The assignment: $f \mapsto-\frac{1}{2} x^{2}, e \mapsto \frac{1}{2}\left(\partial^{2}-\frac{k(k+1)}{x^{2}}\right)$ extends to a welldefined algebra isomorphism $\eta: \mathcal{U} \mathfrak{g} /\left(C-c_{k}\right) \cdot \mathcal{U} \mathfrak{g} \stackrel{\sim}{\longrightarrow} \mathcal{B}_{k}$.

Proof. It is straightforward to check that $\eta$ extends to a surjective algebra homomorphism: $\mathcal{U} \mathfrak{g} \rightarrow \mathcal{B}_{k}$. To show that $\eta$ is an isomorphism, write $M(\mu)$ for the Verma module over $\mathfrak{g}=\mathfrak{s l}_{2}$ with highest weight $\mu$. 
The annihilator of the module $M(k-1 / 2)$ is equal to the ideal $\left(C-c_{k}\right) \cdot \mathcal{U} \mathfrak{g} \subset \mathcal{U} \mathfrak{g}$, so that the algebra $\mathcal{U} \mathfrak{g} /\left(C-c_{k}\right) \cdot \mathcal{U} \mathfrak{g}$ acts faithfully on this Verma module. On the other hand, consider the $\mathcal{B}_{k}$-module spanned by $x^{2 m-k}, m=0,1,2, \ldots$ It is immediate to check that the pullback of this $\mathcal{B}_{k}$-module via $\eta$ is an $\mathfrak{s l}_{2}$-module isomorphic to $M(k-1 / 2)$. It follows that the map $\eta$ is injective.

Remark. It is known (see e.g. St]) that the algebras $\mathcal{U} \mathfrak{g} /(C-c) \cdot \mathcal{U} \mathfrak{g}, c \in \mathbb{C}$, are pairwise nonisomorphic. Therefore, Proposition 8.2 shows that the algebras $\mathcal{B}_{k}$ and $\mathcal{B}_{l}$ are not isomorphic unless $k=l$ or $k=-l-1$. $\diamond$

According to Propositions 8.1 and 8.2, we may view the deformed Harish-Chandra homomorphism $\Phi_{k}: \mathcal{D}(\mathfrak{g})^{\mathfrak{g}} \rightarrow \mathcal{B}_{k}$, as a certain map: $\mathcal{U} \mathfrak{g} \longrightarrow \mathcal{U} \mathfrak{g} /\left(C-c_{k}\right) \cdot \mathcal{U} \mathfrak{g}$. We are going to check, by an explicit calculation of radial parts, that this map coincides with the canonical projection: $\mathcal{U} \mathfrak{g} \rightarrow \mathcal{U} \mathfrak{g} /\left(C-c_{k}\right) \cdot \mathcal{U} \mathfrak{g}$.

For simplicity consider $k \in \mathbb{Z}^{+}$, the general situation being similar. In this case, the zero weight component of an equivariant function: $\mathfrak{g} \rightarrow V_{k}$, in spherical coordinates has the form: $g(r) \cdot P_{k}(\cos \theta)$, where $g(r)$ is a radial function, and $P_{k}$ is the $k$-th Legendre polynomial. Thus, we get: $\Phi_{k}\left(r^{2}\right)=r^{2}, \Phi_{k}(\mathrm{eu})=r \partial_{r}-1$ (because of conjugation by $\delta=$ $r)$. We compute $\Phi_{k}(\Delta)$. In spherical coordinates, the Laplacian $\Delta$, acting on longitudeindependent functions reads

$$
\Delta=\frac{\partial^{2}}{\partial r^{2}}+2 \frac{1}{r} \frac{\partial}{\partial r}+\frac{1}{r^{2}}\left(\frac{\partial^{2}}{\partial \theta^{2}}+\operatorname{cotan} \theta \cdot \frac{\partial}{\partial \theta}\right) .
$$

Legendre polynomials satisfy the differential equation:

$$
\left(\frac{\partial^{2}}{\partial \theta^{2}}+\operatorname{cotan} \theta \cdot \frac{\partial}{\partial \theta}\right) P_{k}(\cos \theta)=-k(k+1) \cdot P_{k}(\cos \theta)
$$

Therefore, for $f=g(r) \cdot P_{k}(\cos \theta)$, we get

$$
\Delta(f)=\left[\left(\frac{1}{r} \circ\left(\frac{\partial^{2}}{\partial r^{2}}-\frac{k(k+1)}{r^{2}}\right) \circ r\right) g\right] \cdot P_{k}(\cos \theta) .
$$

Thus, $\Phi_{k}(\Delta)=\frac{\partial^{2}}{\partial r^{2}}-\frac{k(k+1)}{r^{2}}$ (= Calogero-Moser operator in one variable).

It is obvious now that $\Phi_{k}$ becomes, after the identification of $\mathcal{D}(\mathfrak{g})^{\mathfrak{g}}$ with $\mathcal{U} \mathfrak{g}$, the projection: $\mathcal{U} \mathfrak{g} \rightarrow \mathcal{U} \mathfrak{g} /\left(C-c_{k}\right) \cdot \mathcal{U} \mathfrak{g}$.

We may now verify Theorem 7.3 for $\mathfrak{s l}_{2}$, using the explicit description of the homorphism $\Phi_{k}$ given above. To prove the theorem, we must show that the ideal $(\mathcal{D}(\mathfrak{g})$. $\left.\operatorname{ad}\left(\operatorname{Ann} V_{k}\right)\right)^{\mathfrak{g}}$ contains $C-c_{k}$. It is easy to see that the eigenvalue of $C$ in $V_{k}$ equals $2 k(k+1)$. Thus, $C-2 k(k+1) \in \operatorname{Ann} V_{k}$. One can check by a direct calculation that $\operatorname{ad}(C-2 k(k+1))$ goes to $4\left(C-c_{k}\right)$ under the isomorphism: $\mathcal{D}(\mathfrak{g})^{\mathfrak{g}} \stackrel{\sim}{\longrightarrow} \mathcal{U} \mathfrak{g}$. Thus, the kernel of $\Phi_{k}$ equals $\left(\mathcal{D}(\mathfrak{g}) \cdot \operatorname{ad}\left(\operatorname{Ann} V_{k}\right)\right)^{\mathfrak{g}}$. 


\section{The kernel of the Harish-Chandra homomorphism}

The main technical tool in the proof of Theorem 7.3 is the following result (for $\mathfrak{g}=\mathfrak{g l}_{n}$ )

Theorem 9.1 In $\mathcal{D}(\mathfrak{g})^{\mathfrak{g}}$, one has an equality: $(\mathcal{D}(\mathfrak{g}) \cdot \operatorname{ad} \mathfrak{g})^{\mathfrak{g}}=\left(\mathcal{D}(\mathfrak{g}) \cdot \operatorname{ad}\left(\operatorname{Ann} V_{0}\right)\right)^{\mathfrak{g}}$.

Remark. Recall that according to Theorem 6.8: $(\mathcal{D}(\mathfrak{g}) \cdot \text { ad } \mathfrak{g})^{\mathfrak{g}}=\operatorname{Ker}(\Phi)$, is the kernel of the homomorphism $\Phi_{k=0}$. Thus, Theorem 9.1 insures compatibility of Theorem 6.8 with Theorem 7.3. Notice further that the statement of Theorem 9.1 might look somewhat surprising, since in $\mathcal{U} \mathfrak{g}$ one has a strict inclusion of ideals: $\operatorname{Ann} V_{0} \subsetneq \mathcal{U} \mathfrak{g} \cdot \operatorname{ad} \mathfrak{g}$. $\diamond$

The remainder of this section is devoted to the proof of Theorem 9.1. The argument below is a refined version of the proof of a commutative analog of this theorem, given in Appendix A. Understanding this section, and its geometric meaning, should be easier for the reader after having first read the proof of Theorem 12.1, and Lemma 12.7 of the Appendix.

We introduce some notation. Let $X=\left\|x_{i j}\right\|$ be an $n \times n$-matrix of indeterminates, and $Y=\left\|\partial_{j i}\right\|$ be the corresponding matrix of partial derivatives. For any noncommutative polynomial $Q(x, y)$, let $\underbrace{Q(X, Y)}$ denote the normal ordering of $Q(X, Y)$. That is, each entry of $\underbrace{Q(X, Y)}$ is obtained by first computing the corresponding entry of $Q(X, Y)$ (which is a sum of products of $x_{i j}$ and $\partial_{k l}$ ), and then moving all the $\partial_{k l}$ to the right from $x_{i j}$. In particular, we write: $\underbrace{[X, Y]}=\underbrace{X Y}-\underbrace{Y X}$, where $\underbrace{X Y}=X Y, \underbrace{Y X}=\left(X^{t} Y^{t}\right)^{t}=$ $Y X-n \cdot I d$, and " $t$ " stands for "transposed".

It is known, see e.g. BJ], that the annihilator of $V_{0}$ in $\mathcal{U} \mathfrak{g}$ is generated by the elements

$$
E_{i j} \cdot E_{k l}-E_{i l} \cdot E_{k j}-\delta_{k j} \cdot E_{i l}+\delta_{k l} \cdot E_{i j} .
$$

Therefore, the left ideal $\mathcal{D} A n n:=\mathcal{D}(\mathfrak{g}) \cdot \operatorname{ad}\left(\operatorname{Ann} V_{0}\right) \subset \mathcal{D}(\mathfrak{g})$ is generated by the elements

$$
R_{j k}^{i l}=\underbrace{[X, Y]} j i \cdot \underbrace{[X, Y]} l k-\underbrace{[X}_{l X, Y]} \cdot \underbrace{[X, Y]} j k-\delta_{j k} \cdot \underbrace{[X, Y]} l i+\delta_{l k} \cdot \underbrace{[X, Y]}]_{j i}
$$

Let $I_{\text {ad }}=(\mathcal{D}(\mathfrak{g}) \cdot \operatorname{ad} \mathfrak{g})^{\mathfrak{g}}$, and $I_{0}=\left(\mathcal{D}(\mathfrak{g}) \cdot \operatorname{ad}\left(\operatorname{Ann} V_{0}\right)\right)^{\mathfrak{g}}=\mathcal{D A n n} \cap \mathcal{D}(\mathfrak{g})^{\mathfrak{g}}$. It is clear that $I_{0} \subset I_{\text {ad }}$. Hence, to prove the Theorem it remains to show that any element $D \in I_{\text {ad }}$ vanishes in $\mathcal{D}(\mathfrak{g}) / \mathcal{D}$ Ann.

Below, we view $\mathfrak{g}=\mathfrak{g l}_{n}$ as an associative (matrix) algebra. We also consider the associative algebra $\mathcal{D}(\mathfrak{g}, \mathfrak{g}):=\mathfrak{g} \otimes \mathcal{D}(\mathfrak{g})$ of $\mathfrak{g}$-valued differential operators on $\mathfrak{g}$, equipped with the standard filtration by the order of differential operators. Then, $\operatorname{gr}(\mathcal{D}(\mathfrak{g}, \mathfrak{g}))=$ $\mathbb{C}[\mathfrak{g} \oplus \mathfrak{g}, \mathfrak{g}]$, is the (non-commutative) associative algebra of polynomial maps: $\mathfrak{g} \oplus \mathfrak{g} \rightarrow \mathfrak{g}$. The filtration on $\mathcal{D}(\mathfrak{g}, \mathfrak{g})$ induces a filtration on the subalgebra $\mathcal{D}(\mathfrak{g}, \mathfrak{g})^{\text {ad } \mathfrak{g}} \subset \mathcal{D}(\mathfrak{g}, \mathfrak{g})$ of ad $\mathfrak{g}$-invariant differential operators (with respect to the simultaneous ad $\mathfrak{g}$-action on both the source and the target spaces). 
Lemma 9.3 The vector space $\mathcal{D}(\mathfrak{g}, \mathfrak{g})^{\text {ad } \mathfrak{g}}$ is spanned by operators of the form $\prod_{i=1}^{m} \operatorname{Tr}\left(P_{i}(X, Y)\right) \cdot Q(X, Y), \quad$ where $P_{1}, \ldots, P_{m}, Q$ are noncommutative polynomials.

Proof. We prove the lemma for an ad $\mathfrak{g}$-invariant $\mathfrak{g}$-valued operator $D$ by induction on $d$, the order of $D$. The principal symbol $D_{d}$ of $D$ with respect to the above filtration is a $\mathfrak{g}$-invariant polynomial: $\mathfrak{g} \oplus \mathfrak{g} \rightarrow \mathfrak{g},(X, Y) \mapsto D_{d}(X, Y)$. By Weyl's fundamental theorem of invariant theory $[\mathrm{We}$, this polynomial must be a linear combination of expressions of the form $\prod_{i=1}^{m} \operatorname{Tr}\left(P_{i}(X, Y)\right) \cdot Q(X, Y)$. Therefore, subtracting from $D$ expressions of such form (but now with noncommuting matrix entries), we reduce the order of $D$.

Lemma 9.4 Any element of $I_{\mathrm{ad}}$ is a linear combination of elements of the form: $\prod_{i} \operatorname{Tr}\left(P_{i}(X, Y)\right) \cdot \operatorname{Tr}(Q(X, Y) \cdot \underbrace{[X, Y]})$, where $P_{i}, Q$ are noncommutative polynomials.

Proof. Let $E_{i j}$ be an elementary matrix. It is easy to show that the linear vector field $x \rightarrow\left[E_{i j}, x\right]$ on $\mathfrak{g}$ equals $\underbrace{[X, Y]} j i$. Therefore, the ideal $I_{\text {ad }}$ is the set of $\mathfrak{g}$-invariants in the left ideal in $\mathcal{D}(\mathfrak{g})$ generated by the entries of $\underbrace{[X, Y]}$. Thus, any element of $I_{\text {ad }}$ is of the form $\operatorname{Tr}(Q(X, Y) \cdot[X, Y])$, where $Q$ is an invariant differential operator on $\mathfrak{g}$ with values in $\mathfrak{g}$. Thus the claim follows from Lemma 9.3 .

We say that $D \in I_{\text {ad }}$ has level $\leq d$ if it is a linear combination of elements of the form $\prod_{i} \operatorname{Tr}\left(P_{i}(X, Y)\right) \cdot \operatorname{Tr}(Q(X, Y) \cdot \underbrace{[X, Y]})$ with degree of $Q$ being $\leq d$. By Lemma 9.4, the level is well-defined for all elements $D \in I_{\text {ad }}$. Write: $Q:=Q(X, Y)$, for short.

Lemma 9.5 Let $Q_{1}, Q_{2}$ be noncommutative polynomials of $X, Y$ of degrees $d_{1}, d_{2}$ such that $d_{1}+d_{2} \leq d-4$. Then, in $\mathcal{D}(\mathfrak{g}) / \mathcal{D}$ Ann, cf. (9.2), we have: $\operatorname{Tr}(Q_{1} \cdot \underbrace{[X, Y]} \cdot Q_{2} \cdot \underbrace{[X, Y]})=0$.

Proof. We start with the identity:

$$
\operatorname{Tr}(Q_{1} \cdot \underbrace{[X, Y]} \cdot Q_{2} \cdot \underbrace{[X, Y]})=\sum_{p q r s}\left(Q_{1}\right)_{p q} \cdot \underbrace{[X, Y]} q r \cdot\left(Q_{2}\right)_{r s} \cdot \underbrace{[X, Y]} s p .
$$

Therefore, we have in $\mathcal{D}(\mathfrak{g}) / \mathcal{D}$ Ann:

$$
\operatorname{Tr}(Q_{1} \cdot \underbrace{[X, Y]} \cdot Q_{2} \cdot \underbrace{[X, Y]})=\sum_{p q r s}\left(Q_{1}\right)_{p q} \cdot\left(Q_{2}\right)_{r s} \cdot \underbrace{[X, Y]} q r \cdot \underbrace{[X, Y]} s p
$$

Indeed, this identity holds up to terms arising from reordering the second and the third factors, which have lower level and therefore are zero modulo DAnn (note that all these terms belong to $I_{\mathrm{ad}}$ since they have $\underbrace{[X, Y]}$ on the right end).

Looking at the generators $(9.2)$ of $\mathcal{D}$ Ann, we conclude that in $\mathcal{D}(\mathfrak{g}) / \mathcal{D}$ Ann one has:

$\operatorname{Tr}(Q_{1} \cdot \underbrace{[X, Y]} \cdot Q_{2} \cdot \underbrace{[X, Y]})=\sum_{p q r s}\left(Q_{1}\right)_{p q} \cdot\left(Q_{2}\right)_{r s} \cdot(\underbrace{[X, Y]} s r \cdot \underbrace{[X, Y]_{q p}}+\delta_{q p} \cdot[\underbrace{[X, Y]} s r-\delta_{s p} \cdot \underbrace{[X, Y]} q r)$, 
where $\delta_{a b}=$ Kronecker delta. The latter sum thus splits into three parts. The first part, up to lower level terms, equals $\operatorname{Tr}(Q_{1} \cdot \underbrace{[X, Y]}) \cdot \operatorname{Tr}\left(Q_{2} \cdot[\underbrace{[X, Y]})\right.$. Therefore it is zero in $\mathcal{D}(\mathfrak{g}) / \mathcal{D}$ Ann, by the induction assumption. The other two parts have level lower than $d$, so they also vanish in $\mathcal{D}(\mathfrak{g}) / \mathcal{D}$ Ann.

Lemma 9.6 Let $Q_{1}, Q_{2}$ be noncommutative polynomials of $X, Y$ of degrees $d_{1}, d_{2}$ such that $d_{1}+d_{2} \leq d-4$. Then, in $\mathcal{D}(\mathfrak{g}) / \mathcal{D}$ Ann we have an equality:

$$
\operatorname{Tr}(Q_{1} \cdot X \cdot Y \cdot Q_{2} \cdot \underbrace{[X, Y]})=\operatorname{Tr}(Q_{1} \cdot Y \cdot X \cdot Q_{2} \cdot \underbrace{[X, Y]}) .
$$

Proof. We have

$\operatorname{Tr}(Q_{1} \cdot X \cdot Y \cdot Q_{2} \cdot \underbrace{[X, Y]})=\operatorname{Tr}(Q_{1} \cdot Y \cdot X \cdot Q_{2} \cdot \underbrace{[X, Y]})+\operatorname{Tr}(Q_{1} \cdot \underbrace{[X, Y]} \cdot Q_{2} \cdot \underbrace{[X, Y]})+\ldots$,

where "..." denotes a $\mathfrak{g}$-invariant operator of level $\leq d-1$. By the induction assumption, such an operator is zero in $\mathcal{D}(\mathfrak{g}) / \mathcal{D}$ Ann. By Lemma 9.5, in $\mathcal{D}(\mathfrak{g}) / \mathcal{D}$ Ann one has the equation: $\operatorname{Tr}(Q_{1} \cdot \underbrace{[X, Y]} \cdot Q_{2} \cdot \underbrace{[X, Y]})=0$. The Lemma follows.

Lemma 9.7 For any $a, b, c \geq 0$, in $\mathcal{D}(\mathfrak{g})$, one has the identity:

$$
\operatorname{Tr}(\underbrace{X^{a} Y^{b} X^{c}} \underbrace{[X, Y]})-\operatorname{Tr}(\underbrace{X^{a} Y^{b} X^{c}[X, Y]})=\operatorname{Tr}\left(X^{c}\right) \cdot \operatorname{Tr}\left(X^{a} Y^{b}\right)-\operatorname{Tr}\left(X^{a}\right) \cdot \operatorname{Tr}\left(X^{c} Y^{b}\right) .
$$

Proof. First observe that: $\partial_{j i} \cdot \underbrace{[X, Y]}-\underbrace{\partial_{j i} \cdot[X, Y]}=\left[E_{j i}, Y\right]$. Therefore, we find

$$
\begin{aligned}
& \operatorname{Tr}(\underbrace{X^{a} Y^{b} X^{c}} \underbrace{[X, Y]})-\operatorname{Tr}(\underbrace{X^{a} Y^{b} X^{c}[X, Y]})= \\
& \sum_{b_{1}+b_{2}=b-1} \sum_{p q r s} \underbrace{\left(X^{a} Y^{b_{1}}\right)_{p q} \cdot\left(Y^{b_{2}} X^{c}\right)_{r s} \cdot\left[E_{r q}, Y\right]_{s p}}= \\
& \sum_{b_{1}+b_{2}=b-1} \sum_{p q r s} \underbrace{\left(X^{a} Y^{b_{1}}\right)_{p q} \cdot\left(Y^{b_{2}} X^{c}\right)_{r s} \cdot\left(\delta_{r s} \cdot y_{q p}-\delta_{q p} \cdot y_{s r}\right)}= \\
& \sum_{b_{1}+b_{2}=b-1} \underbrace{\operatorname{Tan}}_{\operatorname{Tr}\left(X^{a} Y^{b_{1}+1}\right) \cdot \operatorname{Tr}\left(Y^{b_{2}} X^{c}\right)-\operatorname{Tr}\left(X^{a} Y^{b_{1}}\right) \cdot \operatorname{Tr}\left(Y^{b_{2}+1} X^{c}\right)} .
\end{aligned}
$$

Proof of Theorem 9.1. We must show that any element $D \in I_{\text {ad }}$ is zero in $\mathcal{D}(\mathfrak{g}) / \mathcal{D}$ Ann.

We will prove that $D=0$ in $\mathcal{D}(\mathfrak{g}) / \mathcal{D}$ Ann by induction in the level of $D$. The base of induction $(d=0)$ is obvious. Assume that the level of $D$ is $d$ and for levels $\leq d-1$ it has been proved that elements of $I_{\text {ad }}$ vanish in $\mathcal{D}(\mathfrak{g}) / \mathcal{D}$ Ann. By Lemma 9.4, we may assume that $D=\operatorname{Tr}(Q(X, Y) \cdot \underbrace{[X, Y]})$, where $Q$ has degree $d$.

Lemma 9.6 shows that it is enough to check the statement of the theorem for the elements: $D_{m}^{\prime}=\operatorname{Tr}\left(Q_{m}\right) \cdot \underbrace{[X, Y]}), m=0, \ldots, d$, where $Q_{m}=\sum_{k=0}^{m} X^{m-k} Y^{d-m} X^{k}$. Let 
$D_{m}=\operatorname{Tr}(\sum_{k=0}^{m} \underbrace{X^{m-k} Y^{d-m} X^{k}} \cdot \underbrace{[X, Y]})$. Clearly, $D_{m}=D_{m}^{\prime}$, up to terms of lower level.

Hence, it suffices to show that $D_{m}=0$. We compute $D_{m}$ by means of Lemma 9.7. Observe that after summation over $0 \leq k \leq m$ (under the trace sign) the terms coming from the RHS of the equation of Lemma 9.7 cancel out. Thus, in $\mathcal{D}(\mathfrak{g})$, we find:

$$
\begin{aligned}
D_{m}= & \sum_{k=0}^{m} \operatorname{Tr}(\underbrace{X^{m-k} \cdot Y^{d-m} \cdot X^{k}} \cdot \underbrace{[X, Y]})=\sum_{k=0}^{m} \operatorname{Tr}(\underbrace{X^{m-k} \cdot Y^{d-m} \cdot X^{k} \cdot[X, Y]})= \\
& \sum_{k=0}^{m} \operatorname{Tr}(\underbrace{Y^{d-m} \cdot X^{k} \cdot[X, Y] \cdot X^{m-k}})=\operatorname{Tr}(\underbrace{\left.Y^{d-m} \cdot X^{m}, Y\right]})=0 . \quad \square
\end{aligned}
$$

\section{Proof of Theorem 7.3}

In this section: $\mathfrak{g}=\mathfrak{g l}_{n}$, and we put $I_{k}:=\left(\mathcal{D}(\mathfrak{g}) \cdot \operatorname{ad}\left(\operatorname{Ann} V_{k}\right)\right)^{\mathfrak{g}}$, and $I_{\text {ad }}:=(\mathcal{D}(\mathfrak{g}) \cdot \operatorname{ad} \mathfrak{g})^{\mathfrak{g}}$.

Proof of Theorem 7.3(i) for $\mathbf{k}=\mathbf{0}$. By Theorem 9.1 and Theorem 6.8, we have $I_{0}=I$. Moreover, it is known that $W$-invariant polynomials on $\mathfrak{h}$ and $W$-invariant differential operators with constant coefficients generate $\mathcal{D}(\mathfrak{h})^{W}$, see [Wa], which implies that $\mathcal{B}_{0}=$ $\mathcal{D}(\mathfrak{h})^{W}$. Thus, the Harish-Chandra homomorphism $\Phi$ gives rise to an isomorphism $\Phi_{0}$ : $\mathcal{D}(\mathfrak{g})^{\mathfrak{g}} / I_{0} \stackrel{\sim}{\longrightarrow} \mathcal{B}_{0}$.

Proof of part (i): general case. For any smooth algebraic variety $X$ with a $\mathbb{C}^{*}$-action, we write $\mathcal{D}(X)(l, m) \subset \mathcal{D}(X)$ for the space of differential operators of order $\leq m$ (with respect to the standard filtration on $\mathcal{D}(X))$ and of homogeneity degree $l$ with respect to the $\mathbb{C}^{*}$-action. Given any subspace $A \subset \mathcal{D}(X)$, we put $A(l, m):=A \cap \mathcal{D}(X)(l, m)$. We will apply these notations for $X=\mathfrak{g}$ and for $X=\mathfrak{h}^{\text {reg }}$, with the natural $\mathbb{C}^{*}$-action by dilations in either case.

Observe that the map $\Phi_{k}$ is compatible both with the filtrations and the gradings, furthermore, for any $m \geq 0$ and $l \in \mathbb{Z}$, the space $\mathcal{D}(\mathfrak{g})(l, m)$ is finite dimensional. We claim that, for generic $k$ (all except countably many), one has:

$$
\left(\operatorname{Im} \Phi_{k}\right)(l, m)=\Phi_{k}\left(\left(\mathcal{D}(\mathfrak{g})^{\mathfrak{g}} / I_{k}\right)(l, m)\right) \quad, \quad \forall m \geq 0, l \in \mathbb{Z} .
$$

To prove this, observe that by Theorem 6.10 we know that the map $\operatorname{gr}\left(\Phi_{0}\right)$ is surjective. This implies that (10.1) holds for $k=0$. Further, by semi-continuity, for generic $k$, one has: $\operatorname{dim} I_{k}(l, m) \geq \operatorname{dim} I_{0}(l, m)$. It follows that if an element $D \in \mathcal{D}(\mathfrak{g})^{\mathfrak{g}}$ has degree exactly $d$ modulo $I_{0}$, it has degree $\leq d$ modulo $I_{k}$, for generic $k$. However, $\Phi_{0}(D)$ has degree exactly $d$, which implies that $\Phi_{k}(D)$ has degree $\geq d$, for generic $k$. This implies that (10.1) must hold for generic $k$.

Recall that, as has been mentioned in the Remark following Theorem 7.3, it is clear that: $\mathcal{B}_{k} \subset \operatorname{Im} \Phi_{k}$, for any $k \in \mathbb{C}$. It follows from equation (10.1) that, for generic $k$ and any $m \geq 0$ and $l \in \mathbb{Z}$, we have: $\mathcal{B}_{k}(l, m) \subset\left(\operatorname{Im} \Phi_{k}\right)(l, m)$. In particular, for generic $k$, we deduce

$$
\begin{aligned}
\operatorname{dim} \mathcal{B}_{k}(l, m) & \leq \operatorname{dim}\left(\operatorname{Im} \Phi_{k}\right)(l, m)=\operatorname{dim} \Phi_{k}\left(\left(\mathcal{D}(\mathfrak{g})^{\mathfrak{g}} / I_{k}\right)(l, m)\right) \\
& \leq \operatorname{dim}\left(\mathcal{D}(\mathfrak{g})^{\mathfrak{g}} / I_{k}\right)(l, m)=\operatorname{dim} \mathcal{D}(\mathfrak{g})^{\mathfrak{g}}(l, m)-\operatorname{dim} I_{k}(l, m) .
\end{aligned}
$$


Further, a semi-continuity argument shows that, for generic $k$, one has: $\operatorname{dim} \mathcal{B}_{k}(l, m) \geq$ $\operatorname{dim} \mathcal{B}_{0}(l, m)$, and $\operatorname{dim} I_{k}(l, m) \geq \operatorname{dim} I_{0}(l, m)$. Therefore, since the theorem holds for $k=0$, the inequalities above yield

$$
\begin{aligned}
\operatorname{dim} \mathcal{B}_{k}(l, m) \geq \operatorname{dim} \mathcal{B}_{0}(l, m) & =\operatorname{dim} \mathcal{D}(\mathfrak{g})^{\mathfrak{g}}(l, m)-\operatorname{dim} I_{0}(l, m) \\
& \geq \operatorname{dim} \mathcal{D}(\mathfrak{g})^{\mathfrak{g}}(l, m)-\operatorname{dim} I_{k}(l, m) .
\end{aligned}
$$

Comparing this with (10.2) shows that, for generic $k$, all the inequalities must be equalities, in particular: $\operatorname{dim} I_{k}(l, m)=\operatorname{dim} I_{0}(l, m)$, and $\operatorname{dim} \mathcal{B}_{k}(l, m)=\operatorname{dim} \mathcal{B}_{0}(l, m)$. Thus, for all $m \geq 0, l \in \mathbb{Z}$, we have: $\operatorname{dim} \mathcal{B}_{k}(l, m)=\operatorname{dim}\left(\mathcal{D}(\mathfrak{g})^{\mathfrak{g}} / I_{k}\right)(l, m)$, and the map $\Phi_{k}$ is an isomorphism, for generic $k$.

It remains to show that the image of $\Phi_{k}$ is $\mathcal{B}_{k}$ not only for generic but actually for all $k \in \mathbb{C}$. It follows from the fundamental theorem of invariant theory (see Wa ) that $\mathbb{C}[\mathfrak{h} \oplus \mathfrak{h}]^{W}$ is generated, as a Poisson algebra, by the two subalgebras $\mathbb{C}[\mathfrak{h}]^{W}$ of invariant polynomials on the first and the second copies of $\mathfrak{h}$, respectively. Therefore, for any $k$, we have $\mathbb{C}[\mathfrak{h} \oplus \mathfrak{h}]^{W} \subset \operatorname{gr}\left(\mathcal{B}_{k}\right)$, where 'gr' is taken with respect to the natural filtration by the order of differential operators. Also, as we mentioned, one has $\mathcal{B}_{0}=\mathcal{D}(\mathfrak{h})^{W}$, hence $\operatorname{gr}\left(\mathcal{B}_{0}\right)=\mathbb{C}[\mathfrak{h} \oplus \mathfrak{h}]^{W}$. Therefore,

$$
\operatorname{dim} \mathcal{B}_{k}(l, m)=\operatorname{dim}\left(\bigoplus_{i \leq m} \operatorname{gr}_{i}\left(\mathcal{B}_{k}(l, m)\right)\right) \geq \operatorname{dim}\left(\bigoplus_{i \leq m} \operatorname{gr}_{i}\left(\mathcal{B}_{0}(l, m)\right)\right)=\operatorname{dim} \mathcal{B}_{0}(l, m) .
$$

On the other hand, since $\mathcal{B}_{k}$ is described by generators, the dimension $\operatorname{dim} \mathcal{B}_{k}(l, m)$ cannot go up at special points $k$ (it can only go down). Therefore, (10.3) yields: $\operatorname{dim} \mathcal{B}_{k}(l, m)=$ $\operatorname{dim} \mathcal{B}_{0}(l, m)$, for all $k$. This together with the fact that $\operatorname{Im} \Phi_{k}=\mathcal{B}_{k}$ for generic $k$, implies that $\operatorname{Im} \Phi_{k}=\mathcal{B}_{k}$, for all $k$.

Proof of part (ii). Write $\mathcal{B}$ for the $\mathbb{C}[k]$-algebra whose specialization at any particular value of the parameter $k$ is the algebra $\mathcal{B}_{k}$. In more detail, treat $k$ as a variable, and view the Calogero-Moser operator $(1.16)$ as an element of $\mathcal{D}\left(\mathfrak{h}^{\text {reg }}\right)^{W} \bigotimes \mathbb{C}[k]$. Let $\mathcal{C}$ denote the centraliser of this operator in $\mathcal{D}\left(\mathfrak{h}^{\text {reg }}\right)_{-}^{W} \otimes \mathbb{C}[k]$, and $\mathcal{B} \subset \mathcal{D}\left(\mathfrak{h}^{\text {reg }}\right)^{W} \otimes \mathbb{C}[k]$ the algebra generated by $\mathcal{C}$ and by the polynomial subalgebra $\mathbb{C}[\mathfrak{h}]^{W} \otimes \mathbb{C}[k] \subset \mathcal{D}(\mathfrak{h})^{W} \otimes \mathbb{C}[k]$.

By part (i), we may think of the family of deformed Harish-Chandra homomorphisms $\Phi_{k}$ as a single $\mathbb{C}[k]$-linear homomorphism: $\mathcal{D}(\mathfrak{g})^{\mathfrak{g}} \otimes \mathbb{C}[k] \rightarrow \mathcal{B}$. Let Ker be the kernel of this homomorphism. Similarly, let $\mathbf{I}$ be the two-sided ideal of the algebra $\mathcal{D}(\mathfrak{g})^{\mathfrak{g}} \bigotimes \mathbb{C}[k]$ that specialises to $I_{k}$ at any particular value of the parameter $k$. We know that $\mathbf{I} \subset \mathbf{K e r}$. The claim of part (ii) amounts to showing that $\mathbf{K e r} / \mathbf{I}$ is supported, as a $\mathbb{C}[k]$-module, at finitely many points.

To prove this, equip $\mathcal{D}(\mathfrak{g})^{\mathfrak{g}} \otimes \mathbb{C}[k]$ with the standard filtration (place $\mathbb{C}[k]$ in degee 0 ). Then, we have: $\operatorname{gr}\left(\mathcal{D}(\mathfrak{g})^{\mathfrak{g}} \bigotimes \mathbb{C}[k]\right)=\mathbb{C}[\mathfrak{g} \oplus \mathfrak{g}]^{\mathfrak{g}} \bigotimes \mathbb{C}[k]$. The RHS here is a finitely generated algebra, since $\mathfrak{g}$ is a reductive Lie algebra. Hence $\mathcal{D}(\mathfrak{g})^{\mathfrak{g}} \otimes \mathbb{C}[k]$ and $\left(\mathcal{D}(\mathfrak{g})^{\mathfrak{g}} \bigotimes \mathbb{C}[k]\right) / \mathbf{I}$ are finitely generated algebras as well. Therefore, the torsion ideal in $\left(\mathcal{D}(\mathfrak{g})^{\mathfrak{g}} \otimes \mathbb{C}[k]\right) / \mathbf{I}$ is finitely generated by the Hilbert basis theorem. Thus, this ideal is supported at finitely many points of Spec $\mathbb{C}[k]$. But, by part (i), for all $k$ except possibly a countable subset, we have: $I_{k}=\operatorname{Ker}\left(\Phi_{k}\right)$. It follows that $\operatorname{Ker} / \mathbf{I}$ is contained in the torsion ideal in $\left(\mathcal{D}(\mathfrak{g})^{\mathfrak{g}} \otimes \mathbb{C}[k]\right) / \mathbf{I}$, and we are done. 
Remarks (i) In the course of the proof of the theorem we have established the following:

The algebra $\mathcal{B}$ is a free $\mathbb{C}[k]$-module, and $\operatorname{gr}(\mathcal{B})=\mathbb{C}[\mathfrak{h} \oplus \mathfrak{h}]^{W} \otimes \mathbb{C}[k]$.

This result has been already proved differently in Part 1 of the paper.

(ii) The conjecture that the ideal $J$, (see the notation of Remark at the end of $\S 6$ ), coincides with its radical $\sqrt{J}$ at the level of $\mathfrak{g}$-invariants would easily imply that $\operatorname{Ker}\left(\Phi_{k}\right)=$ $I_{k}$, for all $k$. Indeed, $\operatorname{gr}\left(I_{k}\right)$ contains $(\sqrt{J})^{\mathfrak{g}}$; so the conjecture would imply that $J^{\mathfrak{g}} \subset$ $\operatorname{gr}\left(I_{k}\right)$ which, by Theorem 6.9, equals $\operatorname{gr}\left(I_{0}\right)$, thus $\operatorname{dim} I_{k}(l, m) \geq \operatorname{dim} I_{0}(l, m)$, and hence $\operatorname{Ker}\left(\Phi_{k}\right)=I_{k} . \diamond$

\section{Calogero-Moser space for wreath-products.}

Let $\left(L, \omega_{L}\right)$ be a 2-dimensional symplectic vector space, and $\Gamma \subset S p(L)$ a finite subgroup. Consider the wreath product $\boldsymbol{\Gamma}_{n}=S_{n} \ltimes \Gamma^{n}$, acting on $V:=L^{\oplus n}=\mathbb{C}^{n} \otimes L$, a symplectic vector space. Given $\gamma \in \Gamma$, we write $\gamma_{i} \in \boldsymbol{\Gamma}_{n}$ for the element $\gamma$ regarded as an element of the $i$-th factor $\Gamma$. Let $s_{i j} \in S_{n}$ denote the transposition: $i \leftrightarrow j$, in $S_{n}$. Then the group $\boldsymbol{\Gamma}_{n}$ is generated by the following symplectic reflections: the transpositions $s_{i j}$, and the elements $\gamma_{i} \in \boldsymbol{\Gamma}_{n}$. The conjugacy classes of symplectic reflections in $\boldsymbol{\Gamma}_{n} \subset S p(V)$ are known to be of the following two types:

(S) The set: $\left\{s_{i j} \cdot \gamma_{i} \cdot \gamma_{j}^{-1}\right\}_{i, j \in[1, n], \gamma \in \Gamma}$, forms a single $\boldsymbol{\Gamma}_{n}$-conjugacy class.

( $\Gamma)$ Elements: $\left\{\gamma_{i}, i \in[1, n], \gamma \in \Gamma \backslash\{1\}\right\}$, form one $\boldsymbol{\Gamma}_{n}$-conjugacy class, for any given conjugacy class of $\gamma$.

An important role below will be played by the conjugacy class $\mathrm{O} \subset \mathfrak{s l}_{n}$, formed by all $n \times n$-matrices of the form: $P-\mathrm{Id}$, where $P$ is a semisimple rank 1 matrix such that $\operatorname{Tr}(P)=\operatorname{Tr}(\mathrm{Id})=n$. Thus, $\mathrm{O}$ is a closed $\mathrm{GL}_{n}$-conjugacy class in $\mathfrak{s l}_{n}$, containing in particular the following matrix:

$$
p=\left(\begin{array}{llllll}
0 & 1 & 1 & \ldots & 1 & 1 \\
1 & 0 & 1 & \ldots & 1 & 1 \\
1 & 1 & 0 & \ldots & 1 & 1 \\
1 & 1 & 1 & \ldots & 0 & 1 \\
1 & 1 & 1 & \ldots & 1 & 0
\end{array}\right)
$$

since $P=\mathrm{p}+\mathrm{Id}$ is a rank one matrix.

Write $\mathbf{e}_{\Gamma} \in \operatorname{End}_{\mathbb{C}}(\mathbb{C} \Gamma)$ for the projector $\mathbf{e}_{\Gamma}: u \mapsto\left(\frac{1}{|\Gamma|} \sum_{\gamma \in \Gamma} \gamma\right) \cdot u$, on the trivial representation of $\Gamma$. Further, given a class function: $\Gamma \backslash\{1\} \rightarrow \mathbb{C}, \gamma \mapsto c_{\gamma}^{\prime}$, let $c^{\prime}=$ $\sum_{\gamma} c_{\gamma}^{\prime} \cdot \gamma$ denote the corresponding central element of $\mathbb{C} \Gamma$, which is traceless in the regular representation. For each pair $c=\left(k, c^{\prime}\right)$, where $k \in \mathbb{C}$ and $c^{\prime}$ is a class function as above, we introduce the following set:

$$
\left.\begin{array}{rl}
M_{\Gamma, n, c}=\{\nabla \in & \operatorname{Hom}_{\Gamma}\left(L, \operatorname{End}_{\mathbb{C}}\left(\mathbb{C}^{n} \otimes \mathbb{C} \Gamma\right)\right) \mid \nabla: x \mapsto \nabla_{x}, \text { such that } \forall x, y \in L \\
& \text { we have: }\left[\nabla_{x}, \nabla_{y}\right] \in \omega_{L}(x, y) \cdot\left(k \cdot|\Gamma| \cdot \mathrm{O} \otimes \mathbf{e}_{\Gamma}+\operatorname{Id}_{\mathbb{C} n} \otimes c^{\prime}\right)
\end{array}\right\}
$$


It is convenient to choose and fix a symplectic basis $\{x, y\}$ in $L$ and this way identify $L$ with $\mathbb{C}^{2}$, so that the group $S p(L)$ gets identified with $\mathrm{SL}_{2}(\mathbb{C})$. Setting $\nabla_{1}:=\nabla_{x}, \nabla_{2}:=$ $\nabla_{y}$, we can rewrite the formula above more concretely as follows:

$$
M_{\Gamma, n, c}=\left\{\nabla_{1}, \nabla_{2} \in \operatorname{End}_{\mathbb{C}}\left(\mathbb{C}^{n} \otimes \mathbb{C} \Gamma\right) \mid \begin{array}{l}
{\left[\nabla_{1}, \nabla_{2}\right]=k \cdot|\Gamma| \cdot \mathbf{o} \otimes \mathbf{e}_{\Gamma}+\operatorname{Id}_{\mathbb{C}^{n}} \otimes c^{\prime},} \\
\text { for some } \mathbf{o} \in \mathrm{O}
\end{array}\right\}
$$

Set $\mathrm{G}_{\Gamma, n}:=\operatorname{Aut}_{\Gamma}\left(\mathbb{C}^{n} \otimes \mathbb{C} \Gamma\right)$ and $\mathrm{PG}_{\Gamma, n}:=\operatorname{PAut}_{\Gamma}\left(\mathbb{C}^{n} \otimes \mathbb{C} \Gamma\right)$. The reductive group $\mathrm{PG}_{\Gamma, n}$ acts naturally on the vector space $\operatorname{Hom}_{\Gamma}\left(L, \operatorname{End}_{\mathbb{C}}\left(\mathbb{C}^{n} \otimes \mathbb{C} \Gamma\right)\right)$ preserving the set $M_{\Gamma, n, c}$.

Definition 11.5 The quotient variety: $\mathcal{M}_{\Gamma, n, c}=M_{\Gamma, n, c} / \mathrm{PG}_{\Gamma, n}$ is called the CalogeroMoser space for $\boldsymbol{\Gamma}_{n}$.

The same variety can be equivalently defined as follows. First take the point $p \in O$, see (11.2), and let $\mathrm{PG}_{\Gamma, n}(\mathrm{p}) \subset \mathrm{PG}_{\Gamma, n}$ denote the isotropy group of the element: $k \cdot|\Gamma| \cdot \mathrm{p} \otimes \mathbf{e}_{\Gamma}+$ $\operatorname{Id}_{\mathbb{C}^{n}} \otimes c^{\prime} \in \operatorname{End}_{\mathbb{C}}\left(\mathbb{C}^{n} \otimes \mathbb{C} \Gamma\right)$. Then, clearly, one has: $\mathcal{M}_{\Gamma, n, c} \simeq M_{\Gamma, n, c}(\mathrm{p}) / \mathrm{PG}_{\Gamma, n}(\mathrm{p})$, where

$$
M_{\Gamma, n, c}(\mathrm{p})=\left\{\nabla_{1}, \nabla_{2} \in \operatorname{End}_{\mathbb{C}}\left(\mathbb{C}^{n} \otimes \mathbb{C} \Gamma\right)\left|\left[\nabla_{1}, \nabla_{2}\right]=k \cdot\right| \Gamma \mid \cdot \mathbf{p} \otimes \mathbf{e}_{\Gamma}+\operatorname{Id}_{\mathbb{C}^{n}} \otimes c^{\prime}\right\}
$$

We describe the structure of the groups $\mathrm{PG}_{\Gamma, n}$ and $\mathrm{PG}_{\Gamma, n}(\mathrm{p})$ in more detail. There is an obvious algebra isomorphism: $\operatorname{End}_{\Gamma}\left(\mathbb{C}^{n} \otimes \mathbb{C} \Gamma\right) \simeq \mathfrak{g l}_{n} \otimes \operatorname{End}_{\Gamma} \mathbb{C} \Gamma \simeq \mathfrak{g l}_{n} \otimes \mathbb{C} \Gamma$. Therefore, decomposing $\mathbb{C} \Gamma$ into irreducible components, identifying $\mathrm{GL}\left(\mathbb{C}^{n} \otimes\right.$ triv) with $\mathrm{GL}_{n}$, and using the notation $\mathrm{G}_{\Gamma, n}^{\prime}:=\prod_{E \in \operatorname{Irrep}(\Gamma) \backslash \text { triv }} \mathrm{GL}\left(\mathbb{C}^{n} \otimes E\right)$, we find

$$
\mathrm{PG}_{\Gamma, n}=\mathrm{G}_{\Gamma, n} / \mathbb{C}^{*}=\left(\prod_{E \in \operatorname{Irrep}(\Gamma)} \mathrm{GL}\left(\mathbb{C}^{n} \otimes E\right)\right) / \mathbb{C}^{*}=\left(\mathrm{GL}_{n} \times \mathrm{G}_{\Gamma, n}^{\prime}\right) / \mathbb{C}^{*}
$$

From this we compute:

$$
\operatorname{dim} \mathrm{PG}_{\Gamma, n}=n^{2}|\Gamma|-1 \quad \text { and } \quad \operatorname{dim} \mathrm{PG}_{\Gamma, n}(\mathrm{p})=n^{2}(|\Gamma|-1)+(n-1)^{2} .
$$

Further, the decomposition: $\mathbb{C}^{n}=\operatorname{Ker}(\mathrm{p}+\mathrm{Id}) \oplus \operatorname{Im}(\mathrm{p}+\mathrm{Id})$ gives an imbedding $\imath$ : $\mathrm{GL}_{n-1} \hookrightarrow \mathrm{GL}_{n}, A \mapsto A \oplus \mathrm{Id}_{\mathrm{Im}(\mathrm{p}+\mathrm{Id})}$. We observe that the composite map:

$$
\mathrm{GL}_{n-1} \times \mathrm{G}_{\Gamma, n}^{\prime} \stackrel{\imath \times \mathrm{Id}}{\longrightarrow} \mathrm{GL}_{n} \times \mathrm{G}_{\Gamma, n}^{\prime} \rightarrow\left(\mathrm{GL}_{n} \times \mathrm{G}_{\Gamma, n}^{\prime}\right) / \mathbb{C}^{*}=\mathrm{PG}_{\Gamma, n}
$$

induces an isomorphism of the group $\mathrm{GL}_{n-1} \times \mathrm{G}_{\Gamma, n}^{\prime}$ with the group $\mathrm{PG}_{\Gamma, n}(\mathrm{p}) \subset \mathrm{PG}_{\Gamma, n}$. We often invert this isomorphism to obtain an imbedding: $\mathrm{PG}_{\Gamma, n}(\mathrm{p}) \stackrel{\sim}{\longrightarrow} \mathrm{GL}_{n-1} \times \mathrm{G}_{\Gamma, n}^{\prime} \hookrightarrow \mathrm{G}_{\Gamma, n}$.

Hamiltonian interpretation The variety $\mathcal{M}_{\Gamma, n, c}$ can be usefully interpreted as follows. The symplectic form on the 2-dimensional space $L$ and the symmetric trace form $\mathrm{Tr}$ : $a, b \mapsto \operatorname{Tr}(a b)$ on $\operatorname{End}_{\mathbb{C}}\left(\mathbb{C}^{n} \otimes \mathbb{C} \Gamma\right)$, make $L^{*} \otimes \operatorname{End}_{\mathbb{C}}\left(\mathbb{C}^{n} \otimes \mathbb{C} \Gamma\right)$, a symplectic vector space with symplectic 2-form $\omega_{L} \otimes \operatorname{Tr}$. Clearly, $\operatorname{Hom}_{\Gamma}\left(L, \operatorname{End}_{\mathbb{C}}\left(\mathbb{C}^{n} \otimes \mathbb{C} \Gamma\right)\right)$ is nothing but the subspace of $\Gamma$-invariants in $L^{*} \otimes \operatorname{End}_{\mathbb{C}}\left(\mathbb{C}^{n} \otimes \mathbb{C} \Gamma\right)$, which is again a symplectic vector space. 
The natural $\mathrm{PG}_{\Gamma, n}$-action on $\operatorname{Hom}_{\Gamma}\left(L\right.$, End $\left._{\mathbb{C}}\left(\mathbb{C}^{n} \otimes \mathbb{C} \Gamma\right)\right)$ is Hamiltonian, and gives rise to a moment map $\mu: \operatorname{Hom}_{\Gamma}\left(L, \operatorname{End}_{\mathbb{C}}\left(\mathbb{C}^{n} \otimes \mathbb{C} \Gamma\right)\right) \longrightarrow\left(\text { Lie } \mathrm{PG}_{\Gamma, n}\right)^{*}$.

It is convenient at this point to introduce three Lie algebras:

$$
\begin{aligned}
& \mathfrak{g}_{\Gamma, n}:=\operatorname{Lie} \mathrm{G}_{\Gamma, n}=\operatorname{End}_{\Gamma}\left(\mathbb{C}^{n} \otimes \mathbb{C} \Gamma\right)=\underset{E \in \operatorname{Irrep}(\Gamma)}{\bigoplus_{\Gamma, n}} \mathfrak{g l}\left(\mathbb{C}^{n} \otimes E\right), \quad \mathfrak{p g}_{\Gamma, n}:=\operatorname{LiePG} \mathrm{PG}_{\Gamma, n}=\mathfrak{g}_{\Gamma, n} / \mathbb{C}, \\
& \mathfrak{s g}_{\Gamma, n}=\left\{a=\left(a_{E}\right)_{E \in \operatorname{lrrep}(\Gamma)} \in \bigoplus_{E \in \operatorname{lrrep}(\Gamma)} \mathfrak{g l}\left(\mathbb{C}^{n} \otimes E\right)=\mathfrak{g}_{\Gamma, n} \mid \sum_{E} \operatorname{Tr}\left(a_{E}\right)=0\right\} .
\end{aligned}
$$

We may identify $\mathfrak{p g}_{\Gamma, n}^{*}$ with $\mathfrak{s g}_{\Gamma, n} \subset \mathfrak{g}_{\Gamma, n}$ via the trace form. Then the moment map above becomes a map $\mu: \operatorname{Hom}_{\Gamma}\left(L, \operatorname{End}_{\mathbb{C}}\left(\mathbb{C}^{n} \otimes \mathbb{C} \Gamma\right)\right) \rightarrow \mathfrak{s g}_{\Gamma, n}$, given by the formula: $\nabla \mapsto\left[\nabla_{1}, \nabla_{2}\right]$.

For $c=\left(k, c^{\prime}\right)$, we set

$\mathbb{O}_{\Gamma, n, c}=\left\{u \in \mathfrak{g l}_{n} \otimes \operatorname{End}_{\mathbb{C}}(\mathbb{C} \Gamma) \quad|\quad u=k \cdot| \Gamma \mid \cdot \mathbf{o} \otimes \mathbf{e}_{\Gamma}+\operatorname{Id}_{\mathbb{C}^{n}} \otimes c^{\prime} \in \mathfrak{g l}_{n} \otimes \operatorname{End}_{\mathbb{C}}(\mathbb{C} \Gamma), \mathbf{o} \in \mathrm{O}\right\}$

which is a coadjoint orbit in $\mathfrak{p g}_{\Gamma, n}^{*} \simeq \mathfrak{s g}_{\Gamma, n}$. By definition we have: $M_{\Gamma, n, c}=\mu^{-1}\left(\mathbb{O}_{\Gamma, n, c}\right)$. Thus, $\mathcal{M}_{\Gamma, n, c}=\mu^{-1}\left(\mathbb{O}_{\Gamma, n, c}\right) / \mathrm{PG}_{\Gamma, n}$, and we get

Proposition 11.10 The variety $\mathcal{M}_{\Gamma, n, c}$ is the Hamiltonian reduction over $\mathbb{O}_{\Gamma, n, c}$ of the symplectic vector space $\operatorname{Hom}_{\Gamma}\left(L, \operatorname{End}_{\mathbb{C}}\left(\mathbb{C}^{n} \otimes \mathbb{C} \Gamma\right)\right)$.

The Proposition below and its proof are completely parallel to [Wi, Prop. 1.7], a result proved by Wilson in the special case: $\Gamma=\{1\}$.

Proposition 11.11 For generic $c \in \mathrm{C}\left(\boldsymbol{\Gamma}_{n}\right)$, the $\mathrm{PG}_{\Gamma, n}$-action on the variety $M_{\Gamma, n, c}$ is free. Furthermore, $\mathcal{M}_{\Gamma, n, c}$ is a smooth symplectic affine algebraic variety of dimension $2 n$.

Proof. We claim first that if $E \subset \mathbb{C}^{n} \otimes \mathbb{C} \Gamma$ is a proper $\Gamma$-stable subspace, which is in addition invariant under $\nabla_{i}, i=1,2$, then $E=0$. If $E \neq 0$, then we consider the operator $\left.\left[\nabla_{1}, \nabla_{2}\right]\right|_{E}$. The trace of this operator is clearly zero. We now use the following observation that will be also exploited many times elsewhere, esp. in Appendix E. Since $\Gamma$ is finite, the character table $\left\|\rho_{i}\left(u_{j}\right)\right\|$, where $\rho_{i}$ is the $i$-th irreducible character of $\Gamma$ and $u_{j}$ is the $j$-th conjugacy class in $\Gamma$, involves a finite number of algebraic numbers. Therefore, vanishing of a linear combination of the $\rho_{i}\left(u_{j}\right)$ 's involving some generic parameters implies that the coefficient in front of each of the $\rho_{i}\left(u_{j}\right)$ 's vanishes identically. Thus, for generic $c=\left(k, c^{\prime}\right)$, vanishing of the trace of $\left.\left[\nabla_{1}, \nabla_{2}\right]\right|_{E}$ implies that the terms in the trace involving $k$, resp. $c^{\prime}$, vanish separately.

Now, looking at $c^{\prime}$-terms we see that $E$ is a multiple of $\mathbb{C} \Gamma$. Further, looking at the $k$-term, we see exactly like in [Wi] that $E^{\Gamma}$, the $\Gamma$-fixed part of $E$, must be $n$-dimensional. It follows that $\operatorname{dim} E=n \cdot|\Gamma|$, hence $E=\mathbb{C}^{n} \otimes \mathbb{C} \Gamma$. Schur lemma implies the freeness of the $\mathrm{PG}_{\Gamma, n}$-action.

We now show that the variety $M_{\Gamma, n, c}$ is smooth. To do this, it is sufficient to show that $k|\Gamma| s \otimes \mathbf{e}_{\Gamma}+1 \otimes c^{\prime}$ is a regular value of the moment map $\mu: \nabla \mapsto\left[\nabla_{1}, \nabla_{2}\right]$, i.e., that the differential $d \mu$ is surjective. But the surjectivity of the differential of a moment map for a group action on a symplectic manifold is well-known to be equivalent to the local freeness of the group action, established above. 
It remains to calculate the dimension of $\mathcal{M}_{\Gamma, n, c}$. First we calculate the dimension of $M_{\Gamma, n, c}(\mathrm{p})$. Since $k \cdot|\Gamma| \cdot \mathrm{p} \otimes \mathbf{e}_{\Gamma}+1 \otimes c^{\prime}$ is a regular value of the moment map, we get

$$
\operatorname{dim} M_{\Gamma, n, c}(\mathrm{p})=\operatorname{dim} \mu^{-1}(\mathrm{p})=\operatorname{dim} \operatorname{Hom}_{\Gamma}\left(L, \operatorname{End}_{\mathbb{C}}\left(\mathbb{C}^{n} \otimes \mathbb{C} \Gamma\right)\right)-\operatorname{dim}\left(\mathfrak{p g}_{\Gamma, n}\right) .
$$

The space $\operatorname{Hom}_{\Gamma}\left(L, \operatorname{End}_{\mathbb{C}}\left(\mathbb{C}^{n} \otimes \mathbb{C} \Gamma\right)\right)$ clearly has dimension $2 n^{2}|\Gamma|$, and, moreover, we have shown above that the $\mathrm{PG}_{\Gamma, n}(\mathrm{p})$-action on $M_{\Gamma, n, c}(\mathrm{p})$ is free. Therefore, using formulas (11.7) and (11.8), we find

$$
\begin{aligned}
\operatorname{dim} \mathcal{M}_{\Gamma, n, c} & =\operatorname{dim} M_{\Gamma, n, c}(\mathrm{p})-\operatorname{dim} \mathrm{PG}_{\Gamma, n}(\mathrm{p})=\left(2 n^{2}|\Gamma|-\operatorname{dim} \mathrm{PG}_{\Gamma, n}\right)-\operatorname{dim} \mathrm{PG}_{\Gamma, n}(\mathrm{p}) \\
& =\left(2 n^{2}|\Gamma|-n^{2}|\Gamma|+1\right)-\left(n^{2}(|\Gamma|-1)+(n-1)^{2}\right)=2 n .
\end{aligned}
$$

Next, recall the quiver variety: $\mathbb{M}_{\Gamma}^{c}(\mathbf{V}) / G_{\Gamma}(\mathbf{V})=\mathfrak{M}_{\Gamma, n, c}$, defined above Lemma 1.12. A straighforward rewriting using McKay correspondence yields the following isomorphisms:

$$
\mathbb{M}_{\Gamma}^{c}(\mathbf{V}) \simeq M_{\Gamma, n, c} \quad, \quad G_{\Gamma}(\mathbf{V}) \simeq \mathrm{PG}_{\Gamma, n} \quad, \quad \mathfrak{M}_{\Gamma, n, c} \simeq \mathcal{M}_{\Gamma, n, c}, \forall c \in Z \Gamma, n \geq 1 .
$$

Corollary 11.13 The variety $\mathcal{M}_{\Gamma, n, c}$ is connected.

Proof. This is a general property of quiver varieties proved by Crawley-Boevey [CB] (proof of a similar result in [Na2] contains a gap).

The algebra $\mathrm{H}_{\kappa}\left(\boldsymbol{\Gamma}_{n}\right)$. According to (11.1), the space $\mathrm{C}\left(\boldsymbol{\Gamma}_{n}\right)$ of class functions on the set of symplectic reflections in $\boldsymbol{\Gamma}_{n}$ can be identified with $Z \Gamma$ via the map assigning to a class function $f \in \mathrm{C}\left(\boldsymbol{\Gamma}_{n}\right)$ the element $k \cdot 1+\sum_{c^{\prime} \in \Gamma \backslash\{1\}} c_{\gamma}^{\prime} \cdot \gamma \in \mathrm{Z} \Gamma$, where $k \in \mathbb{C}$ is the value of $f$ on the conjugacy class of type (S), see (11.1), and $c_{\gamma}^{\prime}$ is the value of $f$ on the corresponding conjugacy class of type $(\Gamma)$. Given $k$ and $c^{\prime}=\sum_{c^{\prime} \in \Gamma \backslash\{1\}} c_{\gamma}^{\prime} \cdot \gamma$ as above, we will write $c=\left(k, c^{\prime}\right) \in \mathrm{C}\left(\boldsymbol{\Gamma}_{n}\right)$ instead of the corresponding class function $f$.

Let $\mathrm{H}_{\kappa}\left(\boldsymbol{\Gamma}_{n}\right), \kappa=\left(t, k, c^{\prime}\right)$, denote the symplectic reflection algebra attached to $\kappa=$ $(t, c) \in \mathbb{C} \times \mathrm{C}\left(\boldsymbol{\Gamma}_{n}\right)$. The algebra $\mathrm{H}_{t, c}\left(\boldsymbol{\Gamma}_{n}\right)$ is generated by elements of the vector space $\mathbb{C}^{n} \otimes L=L^{\oplus n}$, and the group $\boldsymbol{\Gamma}_{n}$. It will be convenient to use the following notation: given $x \in L$, for any $1 \leq k \leq n$, we put: $x_{k}=(0, \ldots, 0, x, 0, \ldots, 0) \in L^{\oplus n}$ ( $x$ placed on the $k$-th spot). Thus, $\mathbb{C} \boldsymbol{\Gamma}_{n}$ and the elements $\left\{x_{1}, \ldots, x_{n}\right\}_{x \in L}$ generate $\mathbf{H}_{t, c}\left(\boldsymbol{\Gamma}_{n}\right)$.

Assume now that $t=0$. By Theorem 1.7 and Theorem 16.1 (Appendix E), we may (and will) view $\operatorname{Spec} \mathrm{Z}_{0, c}\left(\boldsymbol{\Gamma}_{n}\right)$ as the moduli space of simple $\mathrm{H}_{0, c}\left(\boldsymbol{\Gamma}_{n}\right)$-modules.

The map: Irreps $\left(\mathrm{H}_{0, c}\left(\boldsymbol{\Gamma}_{n}\right)\right) \longrightarrow \mathcal{M}_{\Gamma, n, c}$ Let $S_{n-1} \subset S_{n}$ be the subgroup of permutations of the set $\{2, \ldots, n\}$, acting trivially on the label $1 \in\{1, \ldots, n\}$. We have an imbedding: $\boldsymbol{\Gamma}_{n-1}=S_{n-1} \ltimes \Gamma^{n-1} \hookrightarrow S_{n} \ltimes \Gamma^{n}=\boldsymbol{\Gamma}_{n}$. The commutation relations in $\mathrm{H}_{0, c}\left(\boldsymbol{\Gamma}_{n}\right)$, cf. (1.1), imply that, for any $x \in L$, the element $x_{1}=(x, 0, \ldots, 0) \in L^{\oplus n} \subset \mathrm{H}_{t, c}\left(\boldsymbol{\Gamma}_{n}\right)$ commutes with $\boldsymbol{\Gamma}_{n-1} \subset \mathrm{H}_{t, c}\left(\boldsymbol{\Gamma}_{n}\right)$.

Let $E$ be a simple $\mathrm{H}_{0, c}\left(\boldsymbol{\Gamma}_{n}\right)$-module. Write $E^{\boldsymbol{\Gamma}_{n-1}} \subset E$ for the subspace of $\boldsymbol{\Gamma}_{n-1}$-fixed vectors in $E$. The elements $\left\{x_{1}\right\}_{x \in L}$ commute with $\boldsymbol{\Gamma}_{n-1}$, hence preserve the subspace $E^{\Gamma_{n-1}}$, hence, give rise to a $\Gamma$-equivariant map: $L \rightarrow \operatorname{End}\left(E^{\Gamma_{n-1}}\right),\left.x \mapsto x_{1}\right|_{E} \boldsymbol{\Gamma}_{n-1}$. 
Theorem 1.7 says that $\left.E\right|_{\mathbb{C} \Gamma_{n}} \simeq \mathbb{C} \boldsymbol{\Gamma}_{n}$, as a $\boldsymbol{\Gamma}_{n}$-module. It follows that $\operatorname{dim} E^{\boldsymbol{\Gamma}_{n-1}}=n \cdot|\Gamma|$. Moreover, the space $E^{\boldsymbol{\Gamma}_{n-1}}=\left(\mathbb{C} \boldsymbol{\Gamma}_{n}\right)^{\Gamma_{n-1}}$ may be identified with $\mathbb{C}^{n} \otimes \mathbb{C} \Gamma$, as a $\Gamma$-module, as follows. View $\mathbb{C} \boldsymbol{\Gamma}_{n}$ as the vector space of $\mathbb{C}$-valued functions on $\boldsymbol{\Gamma}_{n}$. Then $\left(\mathbb{C} \boldsymbol{\Gamma}_{n}\right)^{\boldsymbol{\Gamma}_{n-1}}$ gets identified with $\mathbb{C}\left[\boldsymbol{\Gamma}_{n-1} \backslash \boldsymbol{\Gamma}_{n}\right]$, the vector space of $\mathbb{C}$-valued functions on $\boldsymbol{\Gamma}_{n-1} \backslash \boldsymbol{\Gamma}_{n}$. By definition, we have a $\boldsymbol{\Gamma}_{n}$-module isomorphism: $\mathbb{C}\left[\boldsymbol{\Gamma}_{n-1} \backslash \boldsymbol{\Gamma}_{n}\right]=\operatorname{Ind}_{\boldsymbol{\Gamma}_{n-1}} \mathbf{1}$. On the other hand, the space $\mathbb{C}^{n} \otimes \mathbb{C} \Gamma=\mathbb{C} \Gamma^{\oplus n}$ has a natural $\boldsymbol{\Gamma}_{n}$-module structure, and there is a canonical $\boldsymbol{\Gamma}_{n}$-module isomorphism: $\mathbb{C}^{n} \otimes \mathbb{C} \Gamma \simeq \operatorname{Ind}_{\boldsymbol{\Gamma}_{n-1}} \boldsymbol{\Gamma}_{n}$. Thus, we get: $E^{\boldsymbol{\Gamma}_{n-1}}=\left(\mathbb{C} \boldsymbol{\Gamma}_{n}\right)^{\boldsymbol{\Gamma}_{n-1}}=$ $\mathbb{C}\left[\boldsymbol{\Gamma}_{n-1} \backslash \boldsymbol{\Gamma}_{n}\right]=\operatorname{Ind}_{\boldsymbol{\Gamma}_{n-1}}^{\boldsymbol{\Gamma}_{n}} \mathbf{1}=\mathbb{C}^{n} \otimes \mathbb{C} \Gamma$. Explicitly, the isomorphism between the RHS and LHS is given by the formulas:

$$
\mathbb{C}^{n} \otimes \mathbb{C} \Gamma \stackrel{\sim}{\longrightarrow}\left(\mathbb{C} \boldsymbol{\Gamma}_{n}\right)^{\Gamma_{n-1}} \simeq E^{\boldsymbol{\Gamma}_{n-1}} \quad, \quad e_{i} \otimes \gamma \mapsto \gamma_{1} \cdot s_{1 i} \in\left(\mathbb{C} \boldsymbol{\Gamma}_{n}\right)^{\boldsymbol{\Gamma}_{n-1}}, \quad \gamma \in \mathbb{C} \Gamma,
$$

where $\left\{e_{i}\right\}$ denotes the standard basis of $\mathbb{C}^{n}$.

Next, recall the matrix $p$, see (11.2).

Lemma 11.15 The endomorphim $\left.\left[x_{1}, y_{1}\right]\right|_{E^{\Gamma_{n-1}}}$ corresponds, under the bijection (11.14), to an endomorphism of $\mathbb{C}^{n} \otimes \mathbb{C} \Gamma$ given by the formula: $\left[x_{1}, y_{1}\right]=k \cdot|\Gamma| \cdot \mathbf{p} \otimes \mathbf{e}_{\Gamma}+\operatorname{Id}_{\mathbb{C}^{n}} \otimes c^{\prime}$.

Proof. Let $x, y \in L$ and $x_{1}=(x, 0, \ldots, 0), y_{1}=(y, 0, \ldots, 0) \in L^{\oplus n}$. Let $E$ be a representation of $\mathrm{H}_{0, c}\left(\boldsymbol{\Gamma}_{n}\right)$ and $E_{1}$ be the space of coinvariants under the subgroup $\boldsymbol{\Gamma}_{n-1}=$ $S_{n-1} \ltimes \Gamma^{n-1}$. Then

$$
\left.\left[x_{1}, y_{1}\right]\right|_{E_{1}}=\sum_{\gamma \in \Gamma, j>1} k \cdot \omega_{s_{1 j} \cdot \gamma_{1} \cdot \gamma_{j}^{-1}}\left(x_{1}, y_{1}\right) \cdot s_{1 j} \cdot \gamma_{1}+\omega_{L}(x, y) \cdot \sum_{\gamma \in \Gamma \backslash\{1\}} c_{\gamma}^{\prime} \cdot \gamma_{1} .
$$

It is easy to calculate that, independently of $\gamma$, one has: $\omega_{s_{1 j} \gamma_{1} \gamma_{j}^{-1}}\left(x_{1}, y_{1}\right)=\omega_{L}(x, y)$.

Now, a key point, verified by a direct calculation, is that the action of the element $\sum_{j>1} s_{1 j}$ on $\mathbb{C}\left[\boldsymbol{\Gamma}_{n-1} \backslash \boldsymbol{\Gamma}_{n}\right]$ goes, under the bijection $\mathbb{C}\left[\boldsymbol{\Gamma}_{n-1} \backslash \boldsymbol{\Gamma}_{n}\right] \stackrel{\sim}{\longrightarrow} \mathbb{C}^{n} \otimes \mathbb{C} \Gamma$, to the operator with matrix $\mathbf{p} \otimes \operatorname{Id}_{\mathbb{C} \Gamma}$ (in the standard basis $\left\{e_{i} \otimes \gamma\right\}_{i=1, \ldots, n, \gamma \in \Gamma}$ of the vector space $\left.\mathbb{C}^{n} \otimes \mathbb{C} \Gamma\right)$. The Lemma now follows since $\sum_{\gamma \in \Gamma} \gamma=|\Gamma| \cdot \mathbf{e}_{\Gamma}$.

By definition of the variety $\mathcal{M}_{\Gamma, n, c}=M_{\Gamma, n, c} / \mathrm{PG}_{\Gamma, n}$, we have: $\mathbb{C}\left[\mathcal{M}_{\Gamma, n, c}\right]=\mathbb{C}\left[M_{\Gamma, n, c}\right]^{\mathrm{PG}_{\Gamma, n}}$. Here, $M_{\Gamma, n, c}$ is an affine subvariety in the vector space $\operatorname{Hom}_{\Gamma}\left(L, \operatorname{End}_{\mathbb{C}}\left(\mathbb{C}^{n} \otimes \mathbb{C} \Gamma\right)\right)$, see (11.3). Thus, $\mathbb{C}\left[\mathcal{M}_{\Gamma, n, c}\right]$ is a quotient of $\mathbb{C}\left[\operatorname{Hom}_{\Gamma}\left(L, \operatorname{End}_{\mathbb{C}}\left(\mathbb{C}^{n} \otimes \mathbb{C} \Gamma\right)\right)\right]^{\mathrm{PG}_{\Gamma, n}}$, the algebra of invariant polynomials. Therefore, the standard increasing filtration on the polynomial algebra (by degree of polynomial) induces an increasing filtration on the algebra $\mathbb{C}\left[\mathcal{M}_{\Gamma, n, c}\right]$. On the other hand, the algebra $\mathrm{H}_{0, c}\left(\boldsymbol{\Gamma}_{n}\right)$ also comes equipped with the canonical increasing filtration.

The main result of this section is the following explicit description of the variety $\operatorname{Spec}\left(Z_{0, c}\left(\boldsymbol{\Gamma}_{n}\right)\right)$, which is a more precise version of Theorem 1.13 .

Theorem 11.16 Let $c \in \mathrm{C}\left(\boldsymbol{\Gamma}_{n}\right)$ be generic. Then 
(i) The morphism: $E \mapsto{ }^{E} \nabla$, assigning to $E \in \operatorname{Irrep}\left(\mathrm{H}_{0, c}\left(\boldsymbol{\Gamma}_{n}\right)\right)$ the $\mathrm{G}_{\Gamma, n}$-conjugacy class of the map ${ }^{E} \nabla: L \rightarrow \operatorname{End}_{\mathbb{C}}\left(E^{\Gamma_{n-1}}\right), x \mapsto{ }^{E} \nabla_{x}:=\left.x_{1}\right|_{E^{\Gamma_{n-1}}}$, induces an isomorphism $\phi: \operatorname{Spec} Z_{0, c}\left(\boldsymbol{\Gamma}_{n}\right) \stackrel{\sim}{\longrightarrow} \mathcal{M}_{\Gamma, n, c}$ of Poisson algebraic varieties.

(ii) The pull-back morphism $\phi^{*}: \mathbb{C}\left[\mathcal{M}_{\Gamma, n, c}\right] \stackrel{\sim}{\longrightarrow} \mathbf{Z}_{0, c}\left(\boldsymbol{\Gamma}_{n}\right)$ is an isomorphism of filtered algebras.

To prove the Theorem we need two lemmas.

Lemma 11.17 Let $A$ be a commutative algebra with unit, and $n \geq 2$ an integer. Then $\left(A^{\otimes n}\right)^{S_{n}}$, the symmetric part of the commutative algebra $A^{\otimes n}$, is generated by elements of the form:

$$
\left\{a_{1}+\ldots+a_{n} \mid a \in A\right\}, \quad \text { where } a_{k}:=1^{\otimes(k-1)} \otimes a \otimes 1^{\otimes(n-k)}, \quad 1 \leq k \leq n .
$$

This lemma is a generalisation of the classical result of $\mathrm{H}$. Weyl [We], who considered the case $A=\mathbb{C}[x, y]$. Weyl's proof applies verbatim in the general case (in the special case $A=\mathbb{C}[x, y]^{\Gamma}$, to be exploited below, the lemma can be found in [Wan, Lemma 1]).

Recall the standard increasing filtration $F_{\bullet} \mathrm{H}_{0, c}\left(\boldsymbol{\Gamma}_{n}\right)$. Given $a \in \mathrm{H}_{0, c}\left(\boldsymbol{\Gamma}_{n}\right)$, we write filt. $\operatorname{deg}(a)=m$ provided $a \in F_{m} \mathrm{H}_{0, c}\left(\boldsymbol{\Gamma}_{n}\right) \backslash F_{m-1} \mathrm{H}_{0, c}\left(\boldsymbol{\Gamma}_{n}\right)$. Let $\mathcal{R}_{\chi}$ denote the simple $\mathrm{H}_{0, c}\left(\boldsymbol{\Gamma}_{n}\right)$-module corresponding to a smooth point $\chi \in \operatorname{Spec} \mathrm{Z}_{0, c}\left(\boldsymbol{\Gamma}_{n}\right)$.

Lemma 11.18 Let $a \in \mathrm{H}_{0, c}\left(\boldsymbol{\Gamma}_{n}\right)$ and $z \in \mathrm{Z}_{0, c}\left(\boldsymbol{\Gamma}_{n}\right)$ be elements such that, for any smooth point $\chi \in \operatorname{Spec} Z_{0, c}$, one has $\left(\left.\operatorname{Tr}\right|_{\mathcal{R}_{\chi}}(a)\right) \cdot \operatorname{Id}=\left.z\right|_{\mathcal{R}_{\chi}}$. Then: filt. $\operatorname{deg}(z) \leq \mathrm{filt} \cdot \operatorname{deg}(a)$.

Proof. Assume the contrary, i.e. let filt. $\operatorname{deg}(a)=m$, and filt. $\operatorname{deg}(z)=m+l$, where $l>0$. Let $z_{0}$ in $\operatorname{gr}_{0, c}\left(\boldsymbol{\Gamma}_{n}\right) \simeq \mathrm{Z}_{0,0}\left(\boldsymbol{\Gamma}_{n}\right)$ be the principal symbol of $z$. Let $E$ be a generic irreducible module over $\mathrm{H}_{0,0}\left(\boldsymbol{\Gamma}_{n}\right)$ such that $\left.z_{0}\right|_{E}$ is not zero. Let $E_{\lambda}$ be a 1parameter deformation of $E$, such that $E_{\lambda}$ is a representation of $\mathrm{H}_{0, \lambda^{2} c}\left(\boldsymbol{\Gamma}_{n}\right)$ for each $\lambda$. Let $f_{\lambda}: \mathrm{H}_{0, c}\left(\boldsymbol{\Gamma}_{n}\right) \stackrel{\sim}{\longrightarrow} \mathrm{H}_{0, \lambda^{2} c}\left(\boldsymbol{\Gamma}_{n}\right), v \mapsto \lambda^{-1} v, v \in V \subset \mathrm{H}_{0, c}\left(\boldsymbol{\Gamma}_{n}\right)$, be the obvious isomorphism (identical on $\mathbb{C} \boldsymbol{\Gamma}_{n}$ ). Then we have

$$
\left.\operatorname{Tr}\right|_{E_{\lambda}}\left(f_{\lambda}(a)\right) \cdot \operatorname{Id}_{E_{\lambda}}=\left.f_{\lambda}(z)\right|_{E_{\lambda}} .
$$

Thus, $\left.\lambda^{m} \cdot \operatorname{Tr}\right|_{E_{\lambda}}\left(f_{\lambda}(a)\right) \cdot \operatorname{Id}_{E_{\lambda}}=\left.\lambda^{m} \cdot f_{\lambda}(z)\right|_{E_{\lambda}}$. But this is a contradiction: the LHS has a finite limit as $\lambda$ goes to 0 , since the element $a$ has degree $m$, while RHS goes to infinity since $\left.f_{\lambda}(z)\right|_{E_{\lambda}}$ is asymptotic to $\left.\lambda^{-m-l} \cdot z_{0}\right|_{E}$.

Proof of Theorem 11.16: We set $\mathrm{H}_{0, c}:=\mathrm{H}_{0, c}\left(\boldsymbol{\Gamma}_{n}\right)$ and $\mathrm{Z}_{0, c}:=\mathrm{Z}_{0, c}\left(\boldsymbol{\Gamma}_{n}\right)$.

Let $f \mapsto \hat{f}$ denote the standard symmetrisation map: Sym $L \rightarrow T L$, and $a \mapsto a_{1}+\ldots$ $+a_{n}$ the map: $T L \rightarrow(T L)^{\otimes n}$, where $a_{k}:=1^{\otimes(k-1)} \otimes a \otimes 1^{\otimes(n-k)}$, as in Lemma 11.17. We form a map: $f \mapsto a_{f}$, given by the following composition:

$$
\operatorname{Sym} L \stackrel{f \mapsto \hat{f}}{\longrightarrow}(T L) \stackrel{a \mapsto a_{1}+\ldots+a_{n}}{\longrightarrow}(T L)^{\otimes n}=T\left(\mathbb{C}^{n} \otimes L\right) \stackrel{\text { projection }}{\longrightarrow} \mathrm{H}_{0, c}\left(\boldsymbol{\Gamma}_{n}\right)
$$


Restricting this map to $\Gamma$-equivariants we obtain a map $\sigma:(\operatorname{Sym} L)^{\Gamma} \rightarrow \mathbf{e H}_{0, c} \mathbf{e}, f \mapsto$ $\sigma(f)=\mathbf{e} a_{f} \mathbf{e}$. We equip the algebra $(\operatorname{Sym} L)^{\Gamma}$ with the filtration induced by the grading. Further, the algebra $\mathbf{e H}_{0, c} \mathbf{e}$ is filtered, and we have a natural isomorphism: $\operatorname{gr}\left(\mathbf{e H}_{0, c} \mathbf{e}\right) \simeq$ $(\operatorname{Sym} V)^{\boldsymbol{\Gamma}_{n}}=\left(\left((\operatorname{Sym} L)^{\Gamma}\right)^{\otimes n}\right)^{S_{n}}$. It is clear, that the map $\sigma$ is filtration preserving, and the associated graded map can be identified with the map: $(\operatorname{Sym} L)^{\Gamma} \rightarrow\left(\left((\operatorname{Sym} L)^{\Gamma}\right)^{\otimes n}\right)^{S_{n}}$ given by the formula: $(\operatorname{gr} \sigma)(f)=f_{1}+\ldots+f_{n}$. Hence, the image of the map gr $\sigma$ generates the algebra $\left(\left((\operatorname{Sym} L)^{\Gamma}\right)^{\otimes n}\right)^{S_{n}}$, by Lemma 11.17 applied to $A=(\operatorname{Sym} L)^{\Gamma}$. It follows that the image of the map $\sigma$ generates the algebra $\mathbf{e H}_{0, c} \mathbf{e}$.

Now choose a basis $\{x, y\}$ in $L$, and identify $\operatorname{Sym} L$ with $\mathbb{C}[x, y]$, a polynomial algebra. We write: $f(x, y) \mapsto \hat{f}(x, y)$ for the corresponding symmetrisation map. Further, compose $\sigma$ with the Satake isomorphism to get a map $\sigma^{\sharp}: \mathbb{C}[x, y]^{\Gamma}=(\operatorname{Sym} L)^{\Gamma} \longrightarrow$ $\mathbf{e H}_{0, c} \mathbf{e} \stackrel{\sim}{\longrightarrow} \mathrm{Z}_{0, c}$.

Let $\mathbf{e}_{\text {diag }}: \mathbb{C}^{n} \rightarrow \mathbb{C}_{\text {diag }} \subset \mathbb{C}^{n}$ denote the projection on the principal diagonal, so that $\mathbf{e}_{\text {diag }} \otimes \mathbf{e}_{\Gamma}$ is the projection to the 1-dimensional subspace $\mathbb{C}_{\text {diag }} \otimes \mathbf{e} \subset \mathbb{C}^{n} \otimes \mathbb{C} \Gamma$, on which $\left[x_{1}, y_{1}\right]=\left[\nabla_{x}, \nabla_{y}\right]=(n-1) \cdot k \cdot|\Gamma|$, and on which $S_{n}$ acts trivially. It follows from the construction that for the map $\phi^{*}: \mathbb{C}\left[\mathcal{M}_{\Gamma, n, c}\right] \rightarrow \mathrm{Z}_{0, c}$ we have:

$$
\phi^{*}\left(\left(\mathbf{e}_{\text {diag }} \otimes \mathbf{e}_{\Gamma}\right) \circ \hat{f}\left(\nabla_{x}, \nabla_{y}\right) \circ\left(\mathbf{e}_{\text {diag }} \otimes \mathbf{e}_{\Gamma}\right)\right)=\frac{1}{n} \cdot \sigma^{\sharp}(f) \quad, \quad \forall f \in \mathbb{C}[x, y]^{\Gamma} .
$$

Since the image of the map $\sigma$ generates the algebra $\mathbf{e H}_{0, c} \mathbf{e}$, it follows from this formula that the map $\phi^{*}$ is surjective.

We now show that $\phi^{*}$ is injective. Assume $\operatorname{Ker}\left(\phi^{*}\right) \neq 0$, and let $Y \subset \mathcal{M}_{\Gamma, n, c}$ be the zero variety of the ideal $\operatorname{Ker}\left(\phi^{*}\right) \subset \mathbb{C}\left[\mathcal{M}_{\Gamma, n, c}\right]$. Thus, $Y$ an affine subscheme in $\mathcal{M}_{\Gamma, n, c}$ and, moreover, $\operatorname{dim} Y<\operatorname{dim} \mathcal{M}_{\Gamma, n, c}=\operatorname{dim} V$, since $\mathcal{M}_{\Gamma, n, c}$ is irreducible, by Corollary 11.13. But then we have: $\operatorname{dim} Y<\operatorname{dim} \operatorname{Spec} \mathrm{Z}_{0, c}(=\operatorname{dim} V)$, hence the map $\phi^{*}: \mathbb{C}[Y]=$ $\mathbb{C}\left[\mathcal{M}_{\Gamma, n, c}\right] / \operatorname{Ker}\left(\phi^{*}\right) \longrightarrow \mathrm{Z}_{0, c}$ cannot be surjective, contrary to what we have proven above.

To prove part (ii) of the Theorem, we note that the filtration on $\mathbb{C}\left[\mathcal{M}_{\Gamma, n, c}\right]$ is such that $\operatorname{deg}\left(\nabla_{x}\right)=\operatorname{deg}\left(\nabla_{y}\right)=1$. The filtration on $Z_{0, c}$ is induced from that on $\mathrm{H}_{0, c}$. It follows directly from the proof of part (i) that the map $\left(\phi^{*}\right)^{-1}: \mathrm{Z}_{0, c} \rightarrow \mathbb{C}\left[\mathcal{M}_{\Gamma, n, c}\right]$ is filtration preserving.

Next, we claim that the map $\phi^{*}$ is also filtration preserving. Indeed, any regular function $f$ on $\mathcal{M}_{\Gamma, n, c}$ such that filt. $\operatorname{deg}(f)=d$ is representable as a sum of products of traces of monomials of $\nabla_{x}$ and $\nabla_{y}$, of total degree at most $d$. So $\phi^{*}(f)$ is an element of the form $\phi^{*}(f)(E)=\operatorname{Tr}_{E}\left(h_{f}\right)$, where $h_{f} \in \mathrm{H}_{0, c}\left(\boldsymbol{\Gamma}_{n}\right)$ is an element of filtration degree $d$. Thus, it remains to check that if $h \in \mathrm{H}_{0, c}\left(\boldsymbol{\Gamma}_{n}\right)$ and $z \in \mathrm{Z}_{0, c}=\mathbb{C}\left[\operatorname{IrrepH} \mathrm{H}_{0, c}\left(\boldsymbol{\Gamma}_{n}\right)\right]$ is the element corresponding to the function: $E \rightarrow \operatorname{Tr}_{E}(h)$, then filt. $\operatorname{deg}(z) \leq$ filt. $\operatorname{deg}(h)$. But this follows from Lemma 11.18 .

To complete the proof, it remains to show that the map $\phi$ respects the Poisson brackets. First of all, the Poisson bracket $\{-,-\}_{\mathcal{M}_{\Gamma, n, c}}$ on the algebra $\mathbb{C}\left[\mathcal{M}_{\Gamma, n, c}\right]$ is easily seen to have filtration degree $\leq-2$, in the sense explained above Lemma 2.26. Transporting this bracket to $Z_{0, c}$ via the isomorphism $\phi^{*}$, we obtain, since the map $\phi^{*}$ is filtration compatible, 
a Poisson bracket $\phi^{*}\{-,-\}_{\mathcal{M}_{\Gamma, n, c}}$ on $Z_{0, c}$ of filtration degree $\leq-2$ again. Hence, Lemma 2.26 implies that there exists a constant $\lambda \in \mathbb{C}$, such that $\phi^{*}\{-,-\}_{\mathcal{M}_{\Gamma, n, c}}=\lambda \cdot\{-,-\}_{\mathrm{z}_{0, c}}$.

We now, consider the line $\mathbb{C} \cdot c \subset \mathbf{C}\left(\boldsymbol{\Gamma}_{n}\right)$. Hence, for any $r \in \mathbb{C}^{*}$ we get similarly: $\phi^{*}\{-,-\}_{\mathcal{M}_{\Gamma, n, r \cdot c}}=\lambda(r) \cdot\{-,-\}_{\mathrm{z}_{0, r \cdot c}}$. Comparing homogeneity degrees of both sides of this equation we conclude that the function : $r \mapsto \lambda(r)$ must be homogeneous of degree zero, hence a constant $\lambda(r)=\lambda$. To compute this constant explicitly, we let $r \rightarrow 0$. Thus, we are reduced to the case $c=0$, which is easy to calculate. Namely, on $\mathfrak{g l}_{n} \oplus \mathfrak{g l}_{n}=\mathfrak{g l}_{n} \otimes L$ introduce the symplectic 2-form $\operatorname{Tr} \otimes \omega_{L}$. Then $\phi^{*} \omega_{\mathrm{z}}=\frac{1}{|\Gamma|} \cdot\left(\operatorname{Tr} \otimes \omega_{L}\right)$, where $\omega_{\mathrm{z}}$ is the 2 -form on Spec $Z_{0, c}$ induced by the (nondegenerate) Poisson structure on $Z_{0, c}$. Thus, the theorem is proved.

Interpretation via Quiver varieties. Let $Q=Q(\Gamma)$ be a quiver obtained by choosing an orientation on the affine Dynkin graph (of ADE type) arising from the group $\Gamma \subset \mathrm{SL}_{2}$ via the McKay correspondence. Thus, the set $\mathcal{V}$ of vertices of $Q$ is identified with the set $\operatorname{Irrep}(\Gamma)$ of the isomorphism classes of simple $\Gamma$-modules, so that the trivial representation corresponds to a special vertex 0 (in a somewhat degenerate case: $\Gamma=1$, the quiver $Q$ has only one (rather than 2) vertex and one edge-loop at this vertex). Given $v \in \mathcal{V}$, we write $V_{v} \in \operatorname{Irrep}(\Gamma)$ for the corresponding $\Gamma$-module.

Let $n$ be a positive integer, and $\mathrm{R} Q_{n}$ the affine variety (a vector space) of representations of $Q$ in the vector space: $\bigoplus_{v \in \mathcal{V}} V_{v}^{\oplus n}$, that is representations having vector space $\mathbb{C}^{n} \otimes V_{v}=V_{v}^{\oplus n}$ at the vertex $v \in \mathcal{V}$. The group $\left(\prod_{v \in \mathcal{V}} \operatorname{GL}\left(\mathbb{C}^{n} \otimes V_{v}\right)\right) / \mathbb{C}^{*}$, which is isomorphic to $\mathrm{PG}_{\Gamma, n}$, acts naturally on $\mathrm{RQ}_{n}$. Recall that Lie $\mathrm{PG}_{\Gamma, n}=\mathfrak{p g}_{\Gamma, n}$ is the quotient of $\mathfrak{g}_{\Gamma, n}=\bigoplus_{v \in \mathcal{V}} \mathfrak{g l}\left(\mathbb{C}^{n} \otimes V_{v}\right)$ by the 1-dimensional subalgebra of scalar matrices, $\mathfrak{p g}_{\Gamma, n}=\mathfrak{g}_{\Gamma, n} / \mathbb{C}$, and $\mathfrak{p g}_{\Gamma, n}^{*} \simeq \mathfrak{s g}_{\Gamma, n}$.

Now, fix an element $c^{\prime}=\sum_{\gamma \in \Gamma \backslash\{1\}} c_{\gamma}^{\prime} \cdot \gamma \in Z \Gamma$. For each vertex $v \in \mathcal{V}$, we introduce the complex number $C_{v}:=\sum_{\gamma \in \Gamma \backslash\{1\}} c_{\gamma}^{\prime} \cdot \operatorname{Tr}\left(\left.\gamma\right|_{V_{v}}\right) \in \mathbb{C}$. In particular, $C_{0}=\sum_{\gamma \in \Gamma \backslash\{1\}} c_{\gamma}^{\prime}$.

The $\mathrm{PG}_{\Gamma, n}$-action on $\mathrm{R} Q_{n}$ induces a Hamiltonian $\mathrm{PG}_{\Gamma, n}$-action on $T^{*}\left(\mathrm{R} Q_{n}\right)$, the cotangent bundle on $\mathrm{R} Q_{n}$. Let $\mu_{\mathrm{R} Q_{n}}: T^{*}\left(\mathrm{R} Q_{n}\right) \longrightarrow\left(\mathrm{Lie}_{\mathrm{PG}} \mathrm{G}_{\Gamma, n}\right)^{*}=\mathfrak{p g}_{\Gamma, n}^{*}$ denote the corresponding moment map. By definition of the constants $\left\{C_{v}\right\}_{v \in \mathcal{V}}$, the total trace of the element $\oplus_{v \in \mathcal{V}} C_{v} \cdot \operatorname{Id}_{\mathbb{C}^{n} \otimes V_{v}} \in \mathfrak{g}_{\Gamma, n}$ equals

$$
\sum_{v \in \mathcal{V}} C_{v} \cdot \operatorname{dim}\left(\mathbb{C}^{n} \otimes V_{v}\right)=n \cdot \sum_{v \in \mathcal{V}} \sum_{\gamma \in \Gamma \backslash\{1\}} c_{\gamma}^{\prime} \cdot \operatorname{Tr}\left(\left.\gamma\right|_{V_{v}}\right) \cdot \operatorname{dim} V_{v}=n \cdot \sum_{\gamma \in \Gamma \backslash\{1\}} c_{\gamma}^{\prime} \cdot \operatorname{Tr}\left(\left.\gamma\right|_{\mathbb{C} \Gamma}\right)
$$

Since the trace of any $\gamma \in \Gamma \backslash\{1\}$ in the regular representation vanishes, we see that $\oplus_{v \in \mathcal{V}} C_{v} \cdot \operatorname{Id}_{\mathbb{C}^{n} \otimes V_{v}} \in \mathfrak{s g}_{\Gamma, n}$. Therefore we may (and will) regard the conjugacy class

$\mathrm{O}_{c}:=\left(k \cdot|\Gamma| \cdot \mathrm{O}+C_{0} \cdot \operatorname{Id}_{\mathbb{C}^{n}}\right) \bigoplus\left(\bigoplus_{v \in \mathcal{V} \backslash\{0\}} C_{v} \cdot \operatorname{Id}_{\mathbb{C}^{n} \otimes V_{v}}\right) \quad \in \quad \mathfrak{s g}_{\Gamma, n} \subset \bigoplus_{i \in \mathcal{V}} \mathfrak{g l}\left(\mathbb{C}^{n} \otimes V_{i}\right)$

as a coadjoint orbit $\mathrm{O}_{c} \subset \mathfrak{p g}_{\Gamma, n}^{*}$, via the trace duality. Then, Proposition 11.10 yields: $\mathcal{M}_{\Gamma, n, c} \simeq \mu_{\mathrm{RQ}{ }_{n}}^{-1}\left(\mathrm{O}_{c}\right) / \mathrm{PG}_{\Gamma, n}$. Thus, Theorem 11.16 says:

$$
\mathrm{Z}_{0, c}\left(\boldsymbol{\Gamma}_{n}\right) \simeq \mathbb{C}\left[\mu_{\mathrm{RQ}_{n}}^{-1}\left(\mathrm{O}_{c}\right) / \mathrm{PG}_{\Gamma, n}\right]=\mathbb{C}\left[\mu_{\mathrm{R} Q_{n}}^{-1}\left(\mathrm{O}_{c}\right)\right]^{\mathrm{PG}_{\Gamma, n}}
$$


$\Gamma$-analogue of the Harish-Chandra homomorphism. We now discuss a quantization of (11.20). Fix $c=\left(k, c^{\prime}\right) \in \mathrm{C}\left(\boldsymbol{\Gamma}_{n}\right)$, and identify $c^{\prime}$ with an element $c^{\prime}=$ $\sum_{\gamma \in \Gamma \backslash\{1\}} c_{\gamma}^{\prime} \cdot \gamma \in \mathrm{Z} \Gamma$. For each $v \in \mathcal{V}$, we have defined the complex number $C_{v}:=$ $\sum_{\gamma \in \Gamma \backslash\{1\}} c_{\gamma}^{\prime} \cdot \operatorname{Tr}\left(\left.\gamma\right|_{V_{v}}\right)$.

Write $x \mapsto x_{v}$ for the projection $\operatorname{pr}_{v}: \mathfrak{g}_{\Gamma, n}=\bigoplus_{i \in \mathcal{V}} \mathfrak{g l}\left(\mathbb{C}^{n} \otimes V_{i}\right) \quad \rightarrow \quad \mathfrak{g l}\left(\mathbb{C}^{n} \otimes V_{v}\right)$. Let $\chi_{c^{\prime}}: \mathfrak{g}_{\Gamma, n} \rightarrow \mathbb{C}$ be a character given by the formula: $x \mapsto \sum_{v \in \mathcal{V}} C_{v} \cdot \operatorname{Tr}\left(\left.x_{v}\right|_{\mathbb{C}^{n} \otimes V_{v}}\right)$. The character $\chi_{c^{\prime}}$ vanishes on the scalar subalgebra $\mathbb{C} \subset \mathfrak{g}_{\Gamma, n}$, hence descends to a well-defined homomorphism $\chi_{c^{\prime}}: \mathfrak{p g}_{\Gamma, n}=\mathfrak{g}_{\Gamma, n} / \mathbb{C} \rightarrow \mathbb{C}$.

In the case of the special vertex ' 0 ' corresponding to the trivial representation, we get a Lie algebra homomorphism $\operatorname{pr}_{0}: \mathfrak{g}_{\Gamma, n} \rightarrow \mathfrak{g l}_{n}=\mathfrak{g l}\left(\mathbb{C}^{n} \otimes\right.$ triv $)$. We compose this homomorphism with the $\mathfrak{g l}_{n}$-representation $V_{k}$, see $\S 7$ and Appendix B, to obtain a representation $\varrho_{k}: \mathfrak{g}_{\Gamma, n} \rightarrow \operatorname{End}_{\mathbb{C}} V_{k}$. Again, since the representation $V_{k}$ vanishes on the center of the Lie algebra $\mathfrak{g l}_{n}$, the representation $\varrho_{k}$ vanishes on the scalar subalgebra $\mathbb{C} \subset \mathfrak{g}_{\Gamma, n}$, hence descends to a well-defined homomorphism $\varrho_{k}: \mathfrak{p g}_{\Gamma, n}=\mathfrak{g}_{\Gamma, n} / \mathbb{C} \rightarrow \operatorname{End}_{\mathbb{C}} V_{k}$. We let $\varrho_{k, c^{\prime}}=\varrho_{k} \otimes \chi_{c^{\prime}}: \mathfrak{p g}_{\Gamma, n} \rightarrow\left(\right.$ End $\left._{\mathbb{C}} V_{k}\right) \otimes \mathbb{C}$ be the tensor product representation, and let Ann $\varrho_{k, c^{\prime}} \subset \mathcal{U}\left(\mathfrak{p g}_{\Gamma, n}\right)$ denote its annihilator in the enveloping algebra of $\mathfrak{p g}_{\Gamma, n}$.

Let $\mathcal{D}\left(\mathrm{R} Q_{n}\right)$ be the algebra of polynomial differential operators on $\mathrm{R} Q_{n}$. The action of the group $\mathrm{PG}_{\Gamma, n}$ on $\mathrm{R} Q_{n}$ gives, by differentiation, a Lie algebra map ad : $\mathfrak{p g}_{\Gamma, n} \rightarrow$ $\mathcal{D}\left(\mathrm{R} Q_{n}\right)$. We will also use a 'shifted' map ad $-\chi_{c^{\prime}}: \mathfrak{p g}_{\Gamma, n} \longrightarrow \mathcal{D}\left(\mathrm{R} Q_{n}\right)$, which is a Lie algebra homomorphism again. The Lie algebra map 'ad' can be uniquely extended to an associative algebra homomorphism ad $: \mathcal{U}\left(\mathfrak{p g}_{\Gamma, n}\right) \longrightarrow \mathcal{D}\left(\operatorname{R} Q_{n}\right)$. Let $\operatorname{ad}\left(\operatorname{Ann} \varrho_{k, c^{\prime}}\right) \subset$ $\mathcal{D}\left(\mathrm{R} Q_{n}\right)$ denote the image of Ann $\varrho_{k, c^{\prime}} \subset \mathcal{U}\left(\mathfrak{p g}_{\Gamma, n}\right)$ under this homomorphism. Similarly, let $\left(\right.$ ad $\left.-\chi_{c^{\prime}}\right)\left(\mathfrak{p g}_{\Gamma, n}\right) \subset \mathcal{D}\left(\mathrm{R} Q_{n}\right)$ denote the image of the Lie algebra $\mathfrak{p g}_{\Gamma, n}$ under the 'shifted' homomorphism.

Remark. In a subsequent publication we plan to prove the following $\Gamma$-analogue of Theorem 9.1.

$$
\left(\mathcal{D}\left(\mathrm{R} Q_{n}\right) \cdot \operatorname{ad}\left(\operatorname{Ann} \varrho_{0, c^{\prime}}\right)\right)^{\mathrm{PG}_{\Gamma, n}}=\left(\mathcal{D}\left(\mathrm{R} Q_{n}\right) \cdot\left(\operatorname{ad}-\chi_{c^{\prime}}\right)\left(\mathfrak{p g}_{\Gamma, n}\right)\right)^{\mathrm{PG}_{\Gamma, n}}
$$

The proof of this result is obtained by extending the argument of $\S 10$. Details will be given elsewhere.

Further, for any $c=\left(k, c^{\prime}\right) \in \mathrm{C}\left(\boldsymbol{\Gamma}_{n}\right)$, set

$$
I_{k, c^{\prime}}:=\left(\mathcal{D}\left(\mathrm{R} Q_{n}\right) \cdot \operatorname{ad}\left(\operatorname{Ann} \varrho_{k, c^{\prime}}\right)\right)^{\mathrm{PG}_{\Gamma, n}}=\left(\mathcal{D}\left(\mathrm{R} Q_{n}\right) \cdot \operatorname{ad}\left(\operatorname{Ann} \varrho_{k, c^{\prime}}\right)\right) \cap \mathcal{D}\left(\mathrm{R}_{n}\right)^{\mathrm{PG}_{\Gamma, n}} .
$$

This is a two-sided ideal in $\mathcal{D}\left(\mathrm{R}_{n}\right)^{\mathrm{PG}_{\Gamma, n}}$, and we conjecture the following $\Gamma$-analogue of the spherical Harish-Chandra isomorphism, see Corollary 7.4.

Conjecture 11.22 For any $c=\left(k, c^{\prime}\right) \in \mathrm{C}\left(\boldsymbol{\Gamma}_{n}\right)$, there is a filtration preserving algebra isomorphism: $\mathcal{D}\left(\mathrm{R}_{n}\right)^{\mathrm{PG}_{\Gamma, n}} / I_{k, c^{\prime}} \stackrel{\sim}{\longrightarrow} \mathbf{e H}_{1, c} \mathbf{e}$. 
This isomorphism should be a quantization of isomorphism (11.20). In the special case: $n=1$ (and arbitrary group $\Gamma \subset \mathrm{SL}_{2}$ ) the conjecture is known to be true, due to a result of Holland [Ho].

Case: $\Gamma=\{1\}$. From now on we will restrict ourselves to the special case of the trivial group $\Gamma$, so that: $\boldsymbol{\Gamma}_{n}=S_{n}, \mathfrak{g}_{\Gamma, n}=\mathfrak{g l}_{n}$, and $\mathrm{PG}_{\Gamma, n}=\mathrm{PGL}_{n}$. In this case the variety $\mathcal{M}_{\Gamma, n, c}$ reduces to

$$
\mathcal{M}_{n}=\left\{\left(\nabla_{1}, \nabla_{2}\right) \in \mathfrak{g l}_{n} \times \mathfrak{g l}_{n} \mid\left[\nabla_{1}, \nabla_{2}\right]+\operatorname{Id}_{\mathbb{C}^{n}}=\operatorname{rank} 1 \text { matrix }\right\} / \operatorname{Ad} \mathrm{PGL}_{n},
$$

the Calogero-Moser space, introduced by Kazhdan-Kostant-Sternberg [KKS and Wilson [Wi] in connection with the Calogero-Moser integrable system. We can also write: $\mathcal{M}_{n}=$ $\mu^{-1}(\mathrm{O}) / \mathrm{PGL}_{n}=\mu^{-1}(\mathrm{p}) / \mathrm{PGL}_{n}(\mathrm{p})$, where $\mu: \mathfrak{g l}_{n} \times \mathfrak{g l}_{n} \rightarrow \mathfrak{s l}_{n},(X, Y) \mapsto[X, Y]$, cf. (11.6).

Consider the trivial vector bundle: $\mathbb{C}^{n} \times \mu^{-1}(\mathrm{p}) \rightarrow \mu^{-1}(\mathrm{p})$, on $\mu^{-1}(\mathrm{p})$. The fiber $\mathbb{C}^{n}$ over a point $(X, Y) \in \mu^{-1}(\mathrm{p})$ carries the tautological action of the matrices $X$ and $Y$, such that $[X, Y]=\mathrm{p}$. The imbedding: $\mathrm{GL}_{n-1} \hookrightarrow \mathrm{GL}_{n}, A \mapsto A \oplus \operatorname{Id}_{\mathrm{Im}(\mathrm{p}+\mathrm{Id})}$, makes $\mathbb{C}^{n}=$ $\operatorname{Ker}(\mathrm{p}+\mathrm{Id}) \oplus \operatorname{Im}(\mathrm{p}+\mathrm{Id})$ a $\mathrm{GL}_{n-1}$-module, and makes the vector bundle: $\mathbb{C}^{n} \times \mu^{-1}(\mathrm{p}) \rightarrow$ $\mu^{-1}(\mathrm{p})$ a $\mathrm{GL}_{n-1}$-equivariant bundle, with $\mathrm{GL}_{n-1}$ acting diagonally on $\mathbb{C}^{n} \times \mu^{-1}(\mathrm{p})$. This $\mathrm{GL}_{n-1}$-equivariant bundle descends to a well-defined algebraic vector bundle $\mathcal{V}_{n}$ on $\mathcal{M}_{n}=$ $\mu^{-1}(\mathrm{p}) / \mathrm{GL}_{n-1}$, to be called the tautological vector bundle.

Write $\mathrm{H}_{0, c}=\mathrm{H}_{0, c}\left(S_{n}\right)$ for the symplectic reflection algebra attached to the Symmetric group $S_{n}$. Recall that the family of simple $\mathrm{H}_{0, c}$-modules forms, by Theorem 1.7, a distinguished vector bundle $\mathcal{R}$ on Spec $Z_{0, c}$. Using the identification $\phi: \operatorname{Spec} Z_{0, c} \stackrel{\sim}{\longrightarrow} \mathcal{M}_{n}$, provided by Theorem 11.16 we transport $\mathcal{R}$ to a vector bundle $\phi_{*} \mathcal{R}$ on $\mathcal{M}_{n}$. This vector bundle carries a canonical $S_{n}$-action along the fibers, and we proceed now to describing some particular $S_{n}$-isotypic components of $\phi_{*} \mathcal{R}$.

For any (not necessarily irreducible) $S_{n}$-module $\chi$, we let $\mathcal{R}_{(\chi)}=\operatorname{Hom}_{S_{n}}(\chi, \mathcal{R})$ denote the $\chi$-isotypic component of the vector bundle $\mathcal{R}$ on Spec $Z_{0, c}$. In particular, take $\chi$ to be the reflection representation in $\mathbb{C}^{n}$, the sum of the irreducible $(n-1)$-dimensional, and the trivial representation 1 of $S_{n}$. Write $\mathcal{R}_{\mathbb{C}^{n}}$ for the corresponding rank $n$ vector bundle on $\operatorname{Spec} Z_{0, c}$.

Proposition 11.24 Under the isomorphism of Theorem 11.10, the tautological vector bundle $\mathcal{V}_{n}$ on $\mathcal{M}_{n}$ gets identified with the vector bundle $\phi_{*} \mathcal{R}_{\mathbb{C}^{n}}$.

Proof. We use an $S_{n}$-module isomorphism: $\mathbb{C}^{n} \simeq \operatorname{Ind}_{S_{n-1}}^{S_{n}}$ 1. By Frobenius reciprocity, this yields

$$
\mathcal{R}_{\mathbb{C}^{n}}=\operatorname{Hom}_{S_{n}}\left(\mathbb{C}^{n}, \mathcal{R}\right)=\operatorname{Hom}_{S_{n}}\left(\operatorname{Ind}_{S_{n-1}}^{S_{n}} \mathbf{1}, \mathcal{R}\right)=\mathcal{R}^{S_{n-1}}
$$

Thus, to prove the Proposition we should identify $\mathcal{R}^{S_{n-1}}$ with the vector bundle on $\mathcal{M}_{n}=\mu^{-1}(\mathrm{p}) / \mathrm{GL}_{n-1}$, see (11.6), arising from the trivial rank $n$ bundle on $\mu^{-1}(\mathrm{p})$.

Next, recall the variety $\operatorname{Rep}_{\mathbb{C W}}\left(\mathrm{H}_{0, c}\right)$, see (3.6). In the special case $\Gamma=S_{n}, c=1$, we get the projection $\pi: \operatorname{Rep}_{\mathbb{C} S_{n}}\left(H_{0, c}\right) \rightarrow \operatorname{Spec} Z_{0, c}$. According to Theorem 3.7 (ii), the 
trivial PAut $S_{n}\left(\mathbb{C} S_{n}\right)$-equivariant vector bundle on $\operatorname{Rep}_{\mathbb{C} S_{n}}\left(\mathrm{H}_{0, c}\right)$ descends to a vector bundle on $\operatorname{Rep}_{\mathbb{C} S_{n}}\left(\mathrm{H}_{0, c}\right) /$ PAut $_{S_{n}}\left(\mathbb{C} S_{n}\right)=$ Spec $\mathrm{Z}_{0, c}$, which is isomorphic to $\mathcal{R}$. Obviously, the pullback $\pi^{*}\left(\mathcal{R}^{S_{n-1}}\right)$ may be identified with the trivial vector bundle on $\operatorname{Rep}_{\mathbb{C} S_{n}}\left(\mathrm{H}_{0, c}\right)$ with fiber $\left(\mathbb{C} S_{n}\right)^{S_{n-1}}$. Thus, the canonical isomorphism $F:\left(\mathbb{C} S_{n}\right)^{S_{n-1}} \stackrel{\sim}{\longrightarrow} \mathbb{C}^{n}$ provided by formula (11.14) (applied in the special case $\Gamma=\{1\}$ ) and the map $\phi$ of Theorem 11.16 give a map of rank $n$ trivial vector bundles

$$
F_{\text {bun }}: \pi^{*}\left(\mathcal{R}^{S_{n-1}}\right)=\operatorname{Rep}_{\mathbb{C} S_{n}}\left(\mathrm{H}_{0, c}\right) \times\left(\mathbb{C} S_{n}\right)^{S_{n-1}} \longrightarrow \mu^{-1}(\mathrm{p}) \times \mathbb{C}^{n} .
$$

Further, the action of the group $\operatorname{Aut}_{S_{n}}\left(\mathbb{C} S_{n}\right)$ on $\left(\mathbb{C} S_{n}\right)^{S_{n-1}}$ may be identified with an action of a subgroup of the group algebra $\mathbb{C} S_{n}$ acting on $\mathbb{C}\left[S_{n-1} \backslash S_{n}\right]$ by means of right translations. The latter action gives, using the vector space isomorphism $F$ and the imbedding: PAut $S_{n}\left(\mathbb{C} S_{n}\right) \hookrightarrow$ Aut $_{S_{n}}\left(\mathbb{C} S_{n}\right)$ constructed below Proposition 3.8, a group homomorphism: PAut $S_{S_{n}}\left(\mathbb{C} S_{n}\right) \rightarrow \mathrm{GL}_{n}$. It is easy to see that the image of this homomorphism preserves the hyperplane: $\mathbb{C}^{n-1}=\left\{\left(x_{1}, \ldots, x_{n}\right) \in \mathbb{C}^{n} \mid x_{1}+\ldots+x_{n}=0\right\} \subset \mathbb{C}^{n}$, and acts identically on the complementary line. Thus, one obtains a (surjective) group homomorphism $F_{\text {group }}$ : PAut $_{S_{n}}\left(\mathbb{C} S_{n}\right) \rightarrow \mathrm{GL}_{n-1}$.

Now, the trivial bundle on the LHS of (11.25) is equivariant with respect to the group PAut $_{S_{n}}\left(\mathbb{C} S_{n}\right)$, while the bundle on the RHS is equivariant with respect to group $\mathrm{GL}_{n-1}$ (using the imbedding (11.9)). It is easy to see that the vector bundle morphism $F_{\text {bun }}$ intertwines the equivariant structures on the left and on the right via the group homomorphism $F_{\text {group }}:$ Aut $_{S_{n}}\left(\mathbb{C} S_{n}\right) \rightarrow \mathrm{GL}_{n-1}$ described in the previous paragraph. It follows that the morphism $F_{\text {bun }}$ descends to a vector bundle isomorphism: $\mathcal{R}^{S_{n-1}} \stackrel{\sim}{\longrightarrow} \mathcal{V}_{n}$.

Remark. In this remark we let $n$ vary, i.e., $n=2,3, \ldots$, and set $\mathcal{R}_{n}:=\mathcal{R}$. Consider the projection $p_{n}: \mathcal{M}_{n} \rightarrow \operatorname{Sym}^{n}(\mathbb{C}),(X, Y) \mapsto\left\{n\right.$-tuple of eigenvalues of $X$. Set $Z_{n}=$ $\left(p_{n}\right)^{-1}(0)$, and let $\mathcal{R}_{n}^{\circ}:=\left.\mathcal{R}_{n}\right|_{Z_{n}}$. Wilson showed, see [Wi, Lemma 7.1], that for any partition $n=n_{1}+\ldots+n_{k}$, and any point $n_{1} \cdot x_{1}+\ldots+n_{k} \cdot x_{k} \in \operatorname{Sym}^{n}(\mathbb{C})$, where $x_{1}, \ldots, x_{k} \in \mathbb{C}$ are pairwise distinct, one has an isomorphism:

$$
\left(p_{n}\right)^{-1}\left(n_{1} \cdot x_{1}+\ldots+n_{k} \cdot x_{k}\right) \simeq Z_{n_{1}} \times \ldots \times Z_{n_{k}} .
$$

In light of our results, one might expect that for any partition $n=n_{1}+\ldots+n_{k}$, there is a natural $S_{n}$-equivariant vector bundle isomorphism (relative to factorisation (11.26)):

$$
\text { Factorisation: }\left.\mathcal{R}_{n}\right|_{\left(p_{n}\right)^{-1}\left(n_{1} \cdot x_{1}+\ldots n_{k} \cdot x_{k}\right)} \simeq \operatorname{Ind}_{S_{n_{1}} \times \ldots \times S_{n_{k}}}^{S_{n}}\left(\mathcal{R}_{n_{1}}^{\circ} \otimes \ldots \otimes \mathcal{R}_{n_{k}}^{\circ}\right) \text {. }
$$

Harish-Chandra homomorphism $\Phi_{\mathbf{0}, c}$. Set $\mathfrak{g}=\mathfrak{g l}_{n}$, and let $\mathcal{M}^{\mathrm{reg}} \subset \mathcal{M}_{n}$ be the open subset formed by conjugacy classes of pairs $(X, Y) \in \mathfrak{g} \times \mathfrak{g}$ such that the matrix $X$ is semisimple. We can represent points of $\mathcal{M}^{\text {reg }}$ by pairs $(X, Y)$ such that $X$ is a diagonal matrix and $([X, Y]+1)_{i j}=1$, for all $i, j$, see (11.2). Such a representation is unique up to permuting diagonal elements. It is easy to show, see [KKS], that if $(X, Y) \in \mathcal{M}^{\text {reg }}$ and $X=\operatorname{diag}\left(x_{1}, \ldots, x_{n}\right)$, then $X \in \mathfrak{h}^{\text {reg }}$, i.e., $x_{i} \neq x_{j}, \forall i \neq j$, and, moreover, we have: $Y=\operatorname{diag}\left(y_{1}, \ldots, y_{n}\right)+\sum_{i \neq j}\left(x_{i}-x_{j}\right)^{-1} E_{i j}$. We regard $x=\left(x_{1}, \ldots, x_{n}\right)$ as a point in $\mathfrak{h}^{\text {reg }} \subset \mathfrak{h}=\mathbb{C}^{n}$, and $y=\left(y_{1}, \ldots, y_{n}\right)$ as a point in $\mathfrak{h}^{*}=\mathbb{C}^{n}$. Then the assignment: 
$(X, Y) \mapsto(x, y)$ gives a well defined map $\pi: \mathcal{M}^{\text {reg }} \rightarrow\left(\mathfrak{h}^{*} \times \mathfrak{h}^{\text {reg }}\right) / S_{n}$, i.e., the image of a point of $\mathcal{M}^{\text {reg }}$ depends only on the conjugacy class of the pair $(X, Y)$.

Recall that $\mathcal{M}_{n}$ has a natural symplectic structure. Also equip $\mathfrak{h}^{*} \times \mathfrak{h}^{\text {reg }}=T^{*}\left(\mathfrak{h}^{\text {reg }}\right)$ with the standard symplectic structure on the cotangent bundle.

Proposition 11.28 ( $\mathbf{W} \mathbf{W}])$ The set $\mathcal{M}^{\text {reg }}$ is Zariski open dense in $\mathcal{M}_{n}$, and the map $\pi: \mathcal{M}^{\text {reg }} \rightarrow\left(\mathfrak{h}^{*} \times \mathfrak{h}^{\mathrm{reg}}\right) / S_{n}$ is a symplectic isomorphism.

Remark. The density claim in the Proposition follows from the irreducibility of $\mathcal{M}_{n}$, which is a special case of Corollary 11.13. $\diamond$

The Proposition above implies that the inverse map $\pi^{-1}:\left(\mathfrak{h}^{*} \times \mathfrak{h}^{\text {reg }}\right) / S_{n} \stackrel{\sim}{\longrightarrow} \mathcal{M}^{\text {reg }} \hookrightarrow$ $\mathcal{M}_{n}$ induces an injective algebra homomorphism $\pi^{\dagger}: \mathbb{C}\left[\mathcal{M}_{n}\right] \hookrightarrow \mathbb{C}\left[\mathfrak{h}^{*} \times \mathfrak{h}^{\text {reg }}\right]^{S_{n}}$. Under this homomorphism, the subalgebra of $\mathbb{C}[\mathfrak{g} \oplus \mathfrak{g}]^{\mathfrak{g}}$, formed by adg-invariant functions in the $X$-variable (first factor $\mathfrak{g}$ ), goes to the subalgebra $\mathbb{C}\left[\mathfrak{h}^{*}\right]^{S_{n}} \subset \mathbb{C}\left[\mathfrak{h}^{*} \times \mathfrak{h}^{\text {reg }}\right]^{S_{n}}$. The function: $(X, Y) \mapsto \operatorname{Tr}\left(Y^{2}\right)$ goes to $\mathrm{L}_{0, c}=\sum_{i} y_{i}^{2}-\sum_{i \neq j}\left(x_{i}-x_{j}\right)^{-2}$, the Calogero-Moser hamiltonian, cf. Definition 4.12. Therefore, the subalgebra of $\mathbb{C}[\mathfrak{g} \oplus \mathfrak{g}]^{\mathfrak{g}}$, formed by adginvariant functions in the $Y$-variable (second factor $\mathfrak{g}$ ), goes to $\mathcal{C}_{0, c}$, the centraliser in $\mathbb{C}\left[\mathfrak{h}^{*} \times \mathfrak{h}^{\text {reg }}\right]_{-}^{S_{n}}$ of the Calogero-Moser hamiltonian.

Recall the subvariety $M \subset \mathfrak{g} \oplus \mathfrak{g}$ defined in (1.22). Let $\mathcal{I}$ be the ideal in $\mathbb{C}[\mathfrak{g} \oplus \mathfrak{g}]$ generated by quadratic functions of the form: $(X, Y) \mapsto \lambda\left(\bigwedge^{2}([X, Y]+\mathrm{Id})\right)$, where $\lambda$ runs over $\left(\Lambda^{2} \mathfrak{g}\right)^{*}$. According to the results of [Wi] cited above, $\mathcal{I}$ is a radical ideal and, moreover, coincides with the ideal of polynomials vanishing at $M$. We set $I_{0, c}=\mathcal{I}^{\mathfrak{g}}$, an ideal in $\mathbb{C}[\mathfrak{g} \oplus \mathfrak{g}]^{\mathfrak{g}}$. By definition, the kernel of the natural projection pr: $\mathbb{C}[\mathfrak{g} \oplus \mathfrak{g}]^{\mathfrak{g}} \rightarrow \mathbb{C}\left[\mathcal{M}_{n}\right]$ equals $I_{0, c}$. Note further that this projection is a homomorphism of Poisson algebras. Composing the projection pr with the homomorphism $\pi^{\dagger}: \mathbb{C}\left[\mathcal{M}_{n}\right] \hookrightarrow \mathbb{C}\left[\mathfrak{h}^{*} \times \mathfrak{h}^{\mathrm{reg}}\right]^{S_{n}}$, one obtains a Poisson algebra homomorphism $\Phi_{0, c}=\pi^{\dagger} \circ \mathrm{pr}: \mathbb{C}[\mathfrak{g} \oplus \mathfrak{g}]^{\mathfrak{g}} \rightarrow \mathbb{C}\left[\mathfrak{h}^{*} \times \mathfrak{h}^{\text {reg }}\right]^{S_{n}}$.

Recall that $\mathcal{B}_{0, c}$ denotes the Poisson subalgebra in $\mathbb{C}\left[\mathfrak{h}^{*} \times \mathfrak{h}^{\text {reg }}\right]^{S_{n}}$ generated, as a Poisson algebra, by $\mathbb{C}\left[\mathfrak{h}^{*}\right]^{S_{n}}$ and $\mathcal{C}_{0, c} \subset \mathbb{C}\left[\mathfrak{h}^{\text {reg }}\right]^{S_{n}}$. We claim that $\operatorname{Im} \Phi_{0, c}=\mathcal{B}_{0, c}$, moreover, we have

Theorem 11.29 (i) The map $\Phi_{0, c}$ gives a Poisson algebra isomorphism

$$
\Phi_{0, c}: \mathbb{C}[\mathfrak{g} \oplus \mathfrak{g}]^{\mathfrak{g}} / I_{0, c}=\mathbb{C}\left[\mathcal{M}_{n}\right] \stackrel{\sim}{\longrightarrow} \mathcal{B}_{0, c} .
$$

(ii) The isomorphism $\phi^{*}: \mathbb{C}\left[\mathcal{M}_{n}\right] \stackrel{\sim}{\longrightarrow} \mathrm{Z}_{0, c}$ of Theorem 11.10 (ii) coincides with the following composite map

$$
\Phi_{0, c}^{\text {spher }}: \mathbb{C}\left[\mathcal{M}_{n}\right]=\mathbb{C}[\mathfrak{g} \oplus \mathfrak{g}]^{\mathfrak{g}} / I_{0, c} \stackrel{\Phi_{0, c}}{\longrightarrow} \mathcal{B}_{0, c} \stackrel{\left(\Theta_{0, c}^{\text {spher }}\right)^{-1}}{\longrightarrow} \mathbf{e H}_{0, c} \mathbf{e} \stackrel{\text { Satake }}{=} \mathrm{Z}_{0, c} .
$$

Remark. The isomorphism of part (i) is a 'commutative analogue' of the Harish-Chandra isomorphism of Corollary 7.4. The isomorphism $\Phi_{0, c}^{\text {spher }}$ of part (ii) yields Theorem 1.23, formulated in the Introduction. $\diamond$

Proof of Theorem 11.29 View the coordinate ring of the variety $\mathcal{M}_{n}$ as a quotient of the algebra $\mathbb{C}[\mathfrak{g} \oplus \mathfrak{g}]^{\mathfrak{g}}$. Thus, for each $k \geq 0$, we regard the function: $X, Y \mapsto \operatorname{Tr}\left(X^{k}\right)$ 
on $\mathfrak{g} \oplus \mathfrak{g}$ as an element $\operatorname{Tr}_{x, k} \in \mathbb{C}\left[\mathcal{M}_{n}\right]$, and the function: $X, Y \mapsto \operatorname{Tr}\left(Y^{k}\right)$, as an element $\operatorname{Tr}_{y, k} \in \mathbb{C}\left[\mathcal{M}_{n}\right]$ Also, to each $k \geq 0$, we attach the elements: $z_{x, k}=\sum_{i} x_{i}^{k}, z_{y, k}=\sum_{i} y_{i}^{k} \in$ $\mathrm{Z}_{0, c}$.

We first claim that

$$
\Phi_{0, c}^{\mathrm{spher}}\left(\operatorname{Tr}_{y, 2}\right)=z_{y, 2} \quad \text { and } \quad \Phi_{0, c}^{\mathrm{spher}}\left(\operatorname{Tr}_{x, k}\right)=z_{x, k} \quad, \quad \forall k \geq 0 .
$$

Here, the equations on the right are immediate. To prove the equation on the left, recall the Calogero-Moser Hamiltonian $\mathrm{L}_{0, c} \in \mathbb{C}\left[\mathfrak{h}^{\text {reg }} \times \mathfrak{h}^{*}\right]$, see Definition 4.12. We know that: $\Theta_{0, c}^{\text {spher }}\left(z_{y, 2}\right)=\mathrm{L}_{0, c}$. On the other hand, a result of $[\mathrm{KKS}]$ says: $\Phi_{0, c}\left(\operatorname{Tr}_{y, 2}\right)=\mathrm{L}_{0, c}$. Thus, $\Phi_{0, c}^{\text {spher }}\left(\operatorname{Tr}_{y, 2}\right)=\left(\Theta_{0, c}^{\text {spher }}\right)^{-1} \circ \Phi_{0, c}\left(\operatorname{Tr}_{y, 2}\right)=z_{y, 2}$, and (11.30) is proved.

Next, given a simple $\mathrm{H}_{0, c}$-module $E$, write $E_{S_{n-1}}$ for the space of $S_{n-1}$-coinvariants. We claim next that, for any $k \geq 0$, we have:

$$
(n-1) ! \cdot \operatorname{Tr}\left(\left.\left(x_{1}\right)^{k}\right|_{E_{S_{n-1}}}\right)=\left.\operatorname{Tr}\right|_{E}\left(x_{1}\right)^{k} \quad, \quad(n-1) ! \cdot \operatorname{Tr}\left(\left.\left(y_{1}\right)^{k}\right|_{E_{S_{n-1}}}\right)=\left.\operatorname{Tr}\right|_{E}\left(y_{1}\right)^{k} .
$$

To prove this, observe that a generic simple $\mathrm{H}_{0, c}$-module $E$ has a basis $\left\{v_{s}, s \in S_{n}\right\}$, such that, for any $s \in S_{n}$ we have: $x_{1}\left(v_{s}\right)=\lambda_{s^{-1}(1)} \cdot v_{s}$, for certain constants: $\lambda_{1}, \ldots, \lambda_{n} \in \mathbb{C}$. This yields the first equation in (11.31). The second is proved similarly by choosing a basis of $E$ adapted to $y_{1}$ instead of $x_{1}$.

Now, each of the central elements $z_{x, k}, z_{y, k} \in \mathrm{Z}_{0, c}$ acts in $E$ by a scalar, say $z_{x, k, E}, z_{y, k, E}$. Using equation (11.31), for any $k \geq 0$, we find

$$
\operatorname{Tr}\left(\left.\left(x_{1}\right)^{k}\right|_{E_{S_{n-1}}}\right)=\frac{1}{(n-1) !} \cdot \operatorname{Tr}_{E}\left(x_{1}\right)^{k}=\frac{1}{n !} \operatorname{Tr}_{E}\left(\sum_{i=1}^{n}\left(x_{i}\right)^{k}\right)=\frac{1}{n !} \operatorname{Tr}_{E}\left(z_{x, k}\right)=z_{x, k, E}
$$

Thus, we have: $\phi^{*}\left(\operatorname{Tr}_{x, k}\right)=z_{x, k} \in \mathrm{Z}_{0, c}$, and similarly: $\phi^{*}\left(\operatorname{Tr}_{y, k}\right)==z_{y, k}$, for any $k \geq 0$. Thus, 11.30 yields:

$$
\phi^{*}\left(\operatorname{Tr}_{y, 2}\right)=\Phi_{0, c}^{\mathrm{spher}}\left(\operatorname{Tr}_{y, 2}\right) \quad \text { and } \quad \phi^{*}\left(\operatorname{Tr}_{x, k}\right)=\Phi_{0, c}^{\mathrm{spher}}\left(\operatorname{Tr}_{x, k}\right) \quad, \quad \forall k \geq 0 .
$$

We claim next that

The elements: $\left\{\operatorname{Tr}_{y, 2} ; \operatorname{Tr}_{x, k}, k \geq 0\right\}$ generate $\mathbb{C}\left[\mathcal{M}_{n}\right]$ as a Poisson algebra. (11.33)

To prove this, consider the standard filtration on $\mathbb{C}\left[\mathcal{M}_{n}\right]$. Clearly, our claim reduces to a similar statement for $\operatorname{gr} \mathbb{C}\left[\mathcal{M}_{n}\right]$. The Poisson algebra gr $\mathbb{C}\left[\mathcal{M}_{n}\right]$ is isomorphic to gr $\mathrm{Z}_{0, c} \simeq \mathbb{C}\left[\mathfrak{h} \oplus \mathfrak{h}^{*}\right]^{S_{n}}$, due to Theorem 11.16. Moreover, we have shown above that the isomorphism $\operatorname{gr}\left(\phi^{*}\right)$ sends $\operatorname{gr}\left(\operatorname{Tr}_{x, k}\right)$ to $\operatorname{gr}\left(z_{x, k}\right)=\sum_{i} x_{i}^{k} \in \mathbb{C}\left[\mathfrak{h}^{*}\right]^{S_{n}}$, and sends $\operatorname{gr}\left(\operatorname{Tr}_{y, 2}\right)$ to $\operatorname{gr}\left(z_{y, 2}\right)=\sum_{i} y_{i}^{2} \in \mathbb{C}[\mathfrak{h}]^{S_{n}}$. Thus, it suffices to show that these elements generate $\mathbb{C}\left[\mathfrak{h} \oplus \mathfrak{h}^{*}\right]^{S_{n}}$ as a Poisson algebra.

To this end, observe first that the set of elements $\left\{\sum_{i} x_{i}^{k}, \sum_{i} y_{i}^{k}\right\}_{k \geq 0}$ generates $\mathbb{C}\left[\mathfrak{h} \oplus \mathfrak{h}^{*}\right]^{S_{n}}$ as a Poisson algebra, by the theorem of H. Weyl cited earlier. Thus, we have only to show that the set above generates the same Lie algebra as the element $\sum_{i} y_{i}^{2}$ 
together with the set $\left\{\sum_{i} x_{i}^{k}\right\}_{k \geq 0}$. But the two elements: $\sum_{i} x_{i}^{2}, \sum_{i} y_{i}^{2}$ generate a Lie subalgebra in $\mathbb{C}\left[\mathfrak{h} \oplus \mathfrak{h}^{*}\right]^{S_{n}}$ isomorphic to $\mathfrak{s l}_{2}$ and, moreover, this Lie algebra gives the standard $\mathfrak{s l}_{2}$-action on $\mathbb{C}\left[\mathfrak{h} \oplus \mathfrak{h}^{*}\right]^{S_{n}}$. It is therefore clear that applying the operator $\left\{\sum_{i} y_{i}^{2},-\right\}$ to $\sum_{i} x_{i}^{k}$ sufficiently many times one gets $\sum_{i} y_{i}^{k}$, for any $k \geq 2$. This proves (11.33).

We can now complete the proof of the Theorem. Since $\Phi_{0, c}\left(\operatorname{Tr}_{y, 2}\right)=\mathrm{L}_{0, c}$, it is immediate from (11.33) that $\operatorname{Im} \Phi_{0, c} \subset \mathcal{B}_{0, c}$. On the other hand, the discussion preceding Theorem 11.29 implies that the map $\Phi_{0, c}$ is injective and contains the algebra $\mathcal{B}_{0, c}$ in its image. This yields part (i) of Theorem 11.29. Part (ii) follows from equations (11.32), (11.33), and the fact that both $\phi^{*}$ and $\Phi_{0, c}^{\text {spher }}$ are Poisson algebra maps.

Remarks (i) Write $\phi_{+}=\phi$ for the isomorphism: Irreps $\left(\mathrm{H}_{0, c}\right) \stackrel{\sim}{\longrightarrow} \mathcal{M}_{n}$ of Theorem 11.16. One can show that replacing in the definition of $\phi_{+}$the space $E^{S_{n-1}} \subset E$ of $S_{n-1}$-invariants by the space of $S_{n-1}$-anti-invariants, one obtains a similar map

$\phi_{-}: \operatorname{Irreps}\left(\mathrm{H}_{0, c}\right) \rightarrow \mathcal{M}_{n}^{-}=\left\{(X, Y) \in \mathfrak{g} \times \mathfrak{g} \mid[X, Y]-\mathrm{Id}_{\mathbb{C}^{n}}=\operatorname{rank} 1\right.$ matrix $\} / \mathrm{Ad} \mathrm{PGL}_{n}$.

Furthermore, one can verify by an explicit computation, that the maps $\phi_{+}$and $\phi_{-}$are related to each other by the formula: $\phi_{-}=\varphi \circ \phi_{+}$, where $\varphi: \mathcal{M}_{n} \stackrel{\sim}{\longrightarrow} \mathcal{M}_{n}^{-}$is an isomorphism of algebraic varieties given by the formula $\varphi:(X, Y) \mapsto\left(X^{t}, Y^{t}\right)$.

(ii) Recall that Corollary 5.7 provides an explicit formula for the Poisson brackets: $\left\{\psi_{k}, \phi_{l}\right\}$, where the "power sum" elements: $\psi_{\ell}, \phi_{\ell} \in \mathrm{Z}_{0, c}, \ell \geq 0$, have been defined in (5.6). For each $k, l \geq 0$, put $z_{k, l}:=\left\{\psi_{k}, \phi_{l}\right\}$. It is easy to show that the set of elements: $\left\{z_{k, l}\right\}_{k, l \geq 0}$ generates $\mathrm{Z}_{0, c}$ as an associative algebra. Moreover, these elements are images of the functions: $(X, Y) \mapsto k \cdot l \cdot \operatorname{Tr}\left(X^{k} Y^{l}\right)$ under the isomorphism $\phi^{*}$. This gives, in principle, a constructive way to identify $\mathrm{Z}_{0, c}$ and $\mathbb{C}\left[\mathcal{M}_{n}\right]$ as associative algebras. $\diamond$

Observe next that the $\mathbb{G}$-action on $\mathrm{H}_{t, c}$ arising from Theorem 5.10 is trivial on $\mathbb{C} W \subset$ $\mathrm{H}_{0, c}$, hence induces a $\mathbb{G}$-action on the $\mathrm{H}_{0, c}$-module $\mathrm{H}_{0, c}$ e. The latter may be viewed, by Theorem 1.23, as a $\mathbb{G}$-action on the vector bundle $\mathcal{R}$ on $\operatorname{Spec} \mathrm{Z}_{0, c}$.

Recall that Berest and Wilson have defined in $[\mathrm{BW}]$ a $\mathbb{G}$-action on the Calogero-Moser variety $\mathcal{M}_{n}$ by the formulas, cf. (5.8):

$$
a_{\tau, \ell}(X, Y)=\left(X+\tau Y^{\ell}, Y\right) \quad \text { and } \quad b_{\tau, \ell}(X, Y)=\left(X, Y+\tau X^{\ell}\right) \quad, \quad(X, Y) \in \mathfrak{g l}_{n} \times \mathfrak{g l}_{n} .
$$

They showed further that the group $\mathbb{G}$ acts transitively on $\mathcal{M}_{n}$.

Repeating the proof of Proposition 7.7 in the 'limiting' case $\kappa=\infty$, we get

Corollary 11.34 (i) The spherical Harish-Chandra homomorphism $\Phi_{\infty}^{\text {spher }}: \mathcal{D}\left(\mathfrak{g l}_{n}\right)^{\mathfrak{g l}_{n}} \rightarrow$ $\mathbf{e H}_{0, c} \mathbf{e}$ intertwines the $\mathbb{G}$-actions, resp. the $\mathbb{L}$-actions, on $\mathcal{D}\left(\mathfrak{g l}_{n}\right)^{\mathfrak{g l}_{n}}$ and on $\mathbf{e H}_{0, c} \mathbf{e}$.

(ii) The (transitive) $\mathbb{G}$-action on $\mathcal{M}_{n}$ lifts to a $\mathbb{G}$-action on the vector bundle $\mathcal{R}$ on $\mathcal{M}_{n}$. A similar result holds for the Lie algebra $\mathbb{L}$.

Example: $\mathfrak{s l}_{\mathbf{2}}$-case. The algebra $\mathrm{H}_{0, c}\left(\mathbf{A}_{\mathbf{1}}\right)$ has 3 generators: $s, x, y$ with defining relations

$$
s^{2}=1, \quad s x=-x s, \quad s y=-y s, \quad[y, x]=-2 s .
$$


In the following proposition, we collect the results about $\mathrm{H}_{0, c}\left(\mathbf{A}_{\mathbf{1}}\right)$. The proofs are straightforward and are left to the reader.

Proposition 11.35 (i) The center of $\mathrm{H}_{0, c}\left(\mathbf{A}_{\mathbf{1}}\right)$ is generated by the 3 elements: $A=$ $x^{2}, B=y^{2}$, and $C=(x y+y x) / 2$, which satisfy the relation: $A B=C^{2}-1$. Thus, Spec $Z_{0, c}$ is a smooth quadric: $\left\{(a, b, c) \in \mathbb{C}^{3} \mid a b=c^{2}-1\right\}$.

(ii) Let $(a, b, c) \in \operatorname{Spec} \mathrm{Z}_{0, c} \subset \mathbb{C}^{3}$. The unique irreducible representation of $\mathrm{H}_{0, c}\left(\mathbf{A}_{\mathbf{1}}\right)$ corresponding to the point $(a, b, c)$ has a basis $\left\{v_{+}, v_{-}\right\}$such that:

$$
s\left(v_{ \pm}\right)= \pm v_{ \pm}, x\left(v_{+}\right)=\alpha \cdot v_{-}, x\left(v_{-}\right)=\beta \cdot v_{+}, y\left(v_{+}\right)=\gamma \cdot v_{-}, y\left(v_{-}\right)=\delta \cdot v_{+},
$$

where $(\alpha, \beta, \gamma, \delta)$ is a solution of the system of equations:

$$
\alpha \beta=a \quad, \quad \gamma \delta=b \quad, \quad \beta \gamma=c+1 \quad, \quad \alpha \delta=c-1,
$$

defined uniquely modulo rescaling transformations: $(\alpha, \beta, \gamma, \delta) \mapsto\left(t \cdot \alpha, t^{-1} \cdot \beta, t \cdot \gamma, t^{-1} \cdot \delta\right)$.

(iii) The vector bundle $\mathcal{R}$ is a direct sum of two line bundles: $\mathcal{R}=\mathbf{e} \mathcal{R} \oplus \mathbf{e}_{-} \mathcal{R}$, where $\mathbf{e} \mathcal{R}=\mathcal{O}$ is the trivial bundle, and $\mathbf{e}_{-} \mathcal{R}$ is isomorphic to the pullback of $\mathcal{O}(1)$ under the fibration $\pi: \operatorname{Spec} Z_{0, c} \rightarrow \mathbb{P}^{1}$, given by: $\pi(a, b, c)=(\beta, \delta)$.

(iv) The Poisson bracket on $\mathcal{M}_{2}$ is induced by a rotation-invariant symplectic form.

\section{Appendix A: Almost commuting matrices.}

In this appendix we discuss a statement in commutative algebra, which is a classical analogue of Theorem 9.1. Throughout this Appendix $\mathfrak{g}=\mathfrak{g l}_{n}$ and $G=\mathrm{GL}_{n}$.

Let $J \subset \mathbb{C}[\mathfrak{g} \oplus \mathfrak{g}]=\mathbb{C}[X, Y]$ be the ideal generated by the matrix entries of $[X, Y] \in$ $\mathfrak{g}=\operatorname{End}\left(\mathbb{C}^{n}\right)$, i.e. by all functions on $\mathfrak{g} \oplus \mathfrak{g}$ of the form: $X, Y \mapsto \lambda([X, Y])$, where $\lambda$ is a linear function on $\mathfrak{g}$. It is clear that the zero variety of $J$ equals $\mathcal{Z}$, the commuting variety. It is not known whether $J=\sqrt{J}$.

Recall next that any linear map $A: \mathbb{C}^{n} \rightarrow \mathbb{C}^{n}$ induces a linear map $\bigwedge^{2} A: \bigwedge^{2} \mathbb{C}^{n} \longrightarrow$ $\bigwedge^{2} \mathbb{C}^{n}$. Let $J_{1} \subset \mathbb{C}[\mathfrak{g} \oplus \mathfrak{g}]=\mathbb{C}[X, Y]$ be the ideal generated by the matrix entries of $\bigwedge^{2}[X, Y] \in \operatorname{End}\left(\bigwedge^{2} \mathbb{C}^{n}\right)$, i.e. all functions on $\mathfrak{g} \oplus \mathfrak{g}$ of the form: $X, Y \mapsto \eta\left(\bigwedge^{2}[X, Y]\right)$, where $\eta$ is a linear function on $\operatorname{End}\left(\bigwedge^{2} \mathbb{C}^{n}\right)$. The zero variety of $J_{1}$ is the variety $\mathcal{Z}_{1}=$ $\{(X, Y) \in \mathfrak{g} \oplus \mathfrak{g} \mid \operatorname{rk}([X, Y]) \leq 1\}$. Again, it is not known (to us) if $J_{1}=\sqrt{J_{1}}$.

We observe that $J_{1} \subset J$, in particular $\mathcal{Z} \subset \mathcal{Z}_{1}$. The classical analog of theorem 9.1 is

\section{Theorem 12.1 $J^{G}=\left(J_{1}\right)^{G}$.}

The proof of the Theorem requires several auxiliary lemmas. We write $X=\left(x_{i j}\right)$, $Y=\left(y_{i j}\right)$ for $n \times n$-matrices, where $x_{i j}, y_{j i}$ are indeterminates.

Lemma 12.2 The space of $\mathfrak{g}$-invariant polynomials on $\mathfrak{g} \oplus \mathfrak{g}$ with values in $\mathfrak{g}$ is spanned by functions of the form: $(X, Y) \mapsto \prod_{i=1}^{m} \operatorname{Tr}\left(P_{i}(X, Y)\right) \cdot Q(X, Y)$, where $P_{1}, \ldots, P_{m}, Q$ are noncommutative polynomials. 
Proof. Immediate from the Weyl fundamental theorem of invariant theory $\mathrm{We}$.

Lemma 12.3 Any element of $J$ is a linear combination of functions of the form: $(X, Y) \mapsto \prod_{i} \operatorname{Tr}\left(P_{i}(X, Y)\right) \cdot \operatorname{Tr}(Q(X, Y) \cdot[X, Y])$, where $P_{i}, Q$ are noncommutative polynomials.

Proof. Any element of $J$ is of the form $\operatorname{Tr}(Q(X, Y) \cdot[X, Y])$, where $Q$ is an invariant polynomial on $\mathfrak{g} \oplus \mathfrak{g}$ with values in $\mathfrak{g}$. Thus the lemma follows from Lemma 12.2 .

By the definition, the ideal $J_{1}$ is generated by the matrix elements of $\bigwedge^{2}([X, Y])$, i.e. by the elements

$$
R_{j k}^{i l}=[X, Y]_{j i} \cdot[X, Y]_{l k}-[X, Y]_{l i} \cdot[X, Y]_{j k} .
$$

We say that $D \in J$ has level $\leq d$ if it is a linear combination of elements of the form $\prod_{i} \operatorname{Tr}\left(P_{i}(X, Y)\right) \cdot \operatorname{Tr}(Q(X, Y) \cdot[X, Y])$ with degree of $Q$ being $\leq d$. By Lemma 12.3, level is defined for all elements $D \in J$.

To prove Theorem 12.1, we must show that any element $D \in J^{G}$ is zero in $\mathbb{C}[\mathfrak{g} \oplus \mathfrak{g}] / J_{1}$. We will prove that $D=0$ in $\mathbb{C}[\mathfrak{g} \oplus \mathfrak{g}] / J_{1}$ by induction in the level of $D$. The base of induction $(d=0)$ is obvious. Assume that the level of $D$ is $d$ and for levels $\leq d-1$ it has been proved that elements of $J$ vanish in $\mathbb{C}[\mathfrak{g} \oplus \mathfrak{g}] / J_{1}$. By Lemma 12.3, we may assume that $D=\operatorname{Tr}(Q(X, Y) \cdot[X, Y])$, where the degree of $Q$ is $d$.

The following lemma plays the main role in the proof of the induction step. It is here that we use the "rank 1" condition. Write $Q_{i}:=Q_{i}(X, Y)$, for short.

Lemma 12.4 Let $Q_{1}, Q_{2}$ be noncommutative polynomials in $X, Y$ of degrees $d_{1}, d_{2}$ such that $d_{1}+d_{2} \leq d-4$. Then in $\mathbb{C}[\mathfrak{g} \oplus \mathfrak{g}] / J_{1}$ one has: $\operatorname{Tr}\left(Q_{1} \cdot[X, Y] \cdot Q_{2} \cdot[X, Y]\right)=0$.

Proof. We write the trace of $Q_{1} \cdot[X, Y] \cdot Q_{2} \cdot[X, Y]$ as the following sum:

$$
\sum_{p q r s}\left(Q_{1}\right)_{p q} \cdot[X, Y]_{q r} \cdot\left(Q_{2}\right)_{r s} \cdot[X, Y]_{s p}=\sum_{p q r s}\left(Q_{1}\right)_{p q} \cdot\left(Q_{2}\right)_{r s} \cdot[X, Y]_{q r} \cdot[X, Y]_{s p}
$$

Using the relations of $J_{1}$, we conclude that in $\mathbb{C}[\mathfrak{g} \oplus \mathfrak{g}] / J_{1}$ one has:

$\operatorname{Tr}\left(Q_{1} \cdot[X, Y] \cdot Q_{2} \cdot[X, Y]\right)=\sum_{p q r s}\left(Q_{1}\right)_{p q} \cdot\left(Q_{2}\right)_{r s} \cdot[X, Y]_{s r} \cdot[X, Y]_{q p}=\operatorname{Tr}\left(Q_{1} \cdot[X, Y]\right) \cdot \operatorname{Tr}\left(Q_{2} \cdot[X, Y]\right)$,

which is zero in $\mathbb{C}[\mathfrak{g} \oplus \mathfrak{g}] / J_{1}$ by the induction hypothesis.

Lemma 12.5 Let $Q_{1}, Q_{2}$ be noncommutative polynomials in $X, Y$ of degrees $d_{1}, d_{2}$ such that $d_{1}+d_{2} \leq d-4$. Then in $\mathbb{C}[\mathfrak{g} \oplus \mathfrak{g}] / J_{1}$ one has: $\operatorname{Tr}\left(Q_{1} \cdot X \cdot Y \cdot Q_{2} \cdot[X, Y]\right)=$ $\operatorname{Tr}\left(Q_{1} \cdot Y \cdot X \cdot Q_{2} \cdot[X, Y]\right)$. 
Proof. We have

$$
\operatorname{Tr}\left(Q_{1} \cdot X \cdot Y \cdot Q_{2} \cdot[X, Y]\right)=\operatorname{Tr}\left(Q_{1} \cdot Y \cdot X \cdot Q_{2} \cdot[X, Y]\right)+\operatorname{Tr}\left(Q_{1} \cdot[X, Y] \cdot Q_{2} \cdot[X, Y]\right),
$$

By Lemma 12.4, $\operatorname{Tr}\left(Q_{1} \cdot[X, Y] \cdot Q_{2} \cdot[X, Y]\right)$ is zero in $\mathbb{C}[\mathfrak{g} \oplus \mathfrak{g}] / J_{1}$. The lemma is proved.

Proof of Theorem 12.1. The last lemma shows that in order to prove the theorem it is enough to check the statement of the theorem for the elements $D_{m}=\operatorname{Tr}\left(Q_{m} \cdot[X, Y]\right)$, $m=0, \ldots, d$, where $Q_{m}=\sum_{k=0}^{m} X^{m-k} Y^{d-m} X^{k}$. Thus, the theorem follows from the equation

$$
\begin{aligned}
D_{m} & =\sum_{k=0}^{m} \operatorname{Tr}\left(X^{m-k} Y^{d-m} X^{k}[X, Y]\right) \\
& =\sum_{k=0}^{m} \operatorname{Tr}\left(Y^{d-m} X^{k}[X, Y] X^{m-k}\right)=\operatorname{Tr}\left(\left[Y^{d-m} X^{m}, Y\right]\right)=0 .
\end{aligned}
$$

Corollary $12.6\left(\sqrt{J_{1}}\right)^{G}=(\sqrt{J})^{G}$.

There is a more geometric proof of Corollary 12.6, which does not use theorem 12.1. It is based on the following beautiful linear algebraic lemma, see e.g., [Gu]:

Lemma 12.7 Let $A, B$ be two $n \times n$-matrices whose commutator, $[A, B]$ is a rank 1 matrix. Then $A, B$ can be simultaneously conjugated into upper triangular matrices.

The proof of this lemma in [Gu is rather complicated, so we would like to give a simpler proof which we learned from A. Rudakov.

Proof. Without loss of generality we can suppose that the kernel of $A$ is non-zero; otherwise we can replace $A$ by $A-\lambda \cdot \mathrm{Id}$, where $\lambda$ is an eigenvalue of $A$. It is enough to show that there is a proper subspace which is both $A$ - and $B$-stable. Put $C:=[A, B]$.

If Ker $A$ belongs $\operatorname{Ker} C$ then $\operatorname{Ker} A$ is $B$-invariant: $A B x=B A x+C x=0$. Thus, $\operatorname{Ker} A$ is the needed subspace.

If $\operatorname{Ker} A$ is not in $\operatorname{Ker} C$ then there exists $z$ such that $A z=0, C z \neq 0$. This means $A B z=C z$ is not zero. Hence $\operatorname{Im} C$, which is a 1-dimensional space, is contained in $\operatorname{Im} A$. Now $\operatorname{Im} A$ is $B$-invariant, because $B A x=A B x+C x$, and both summands are in $\operatorname{Im} A$. Thus, $\operatorname{Im} A$ is the needed subspace.

We observe now that Lemma 12.7 implies Corollary 12.6. In more detail, recall the variety $\mathcal{Z}_{1}=\{(X, Y) \in \mathfrak{g} \oplus \mathfrak{g} \mid \operatorname{rk}([X, Y]) \leq 1\}$. It follows from Lemma 12.7 that the closure of any $G$-orbit in $\mathcal{Z}_{1}$ contains a point of $\mathcal{Z}$ (since a pair of upper triangular matrices can be conjugated arbitrarily close to their diagonal parts). Hence, any $\mathfrak{g}$-invariant polynomial on $\mathfrak{g} \oplus \mathfrak{g}$ that vanishes on $\mathcal{Z}$, must vanish on the whole of $\mathcal{Z}_{1}$.

Remarks (i) We have shown that Lemma 12.7 implies Corollary 12.6. In fact, the corollary also easily implies the lemma. Indeed, it is easy to show that any two matrices the 
closure of whose $G L_{n}$-orbit contains a pair of commuting matrices, generate a solvable Lie algebra.

(ii) There is a natural analogue of the claim of Lemma 12.7 for a general semisimple Lie algebra $\mathfrak{g}$ saying that any pair of elements $A, B \in \mathfrak{g}$ such that $[A, B]$ is a root element, generates a solvable Lie algebra. This claim is false in such generality; it is false for the Lie algebra $\mathfrak{g}=\mathfrak{s o}_{8}$, for instance.

\section{Appendix B: Geometric construction of $V_{k}$}

In this appendix we explain the geometric construction of the representation $V_{k}$ over $\mathfrak{g}=\mathfrak{s l}_{n}$ using the notion of twisted differential operators, see e.g., [BB].

Let $\omega$ be the canonical (line) bundle on $\mathbb{P}^{n-1}$, and let $\mathbf{L}$ denote its total space with zero-section removed. Thus, $\mathbf{L}$ is a principal $\mathbb{C}^{*}$-bundle on $\mathbb{P}^{n-1}$. Write eu for the Euler vector field on $\mathbf{L}$ generating the $\mathbb{C}^{*}$-action, and $\mathcal{D}(\mathbf{L})^{\text {eu }}$ for the centralizer of the Euler field in the algebra of global algebraic differential operators on $\mathbf{L}$. Note that eu is a central element in $\mathcal{D}(\mathbf{L})^{\mathrm{eu}}$. For any $k \in \mathbb{C}$, we set $\mathcal{D}_{k}(\mathbf{L}):=\mathcal{D}(\mathbf{L})^{\mathrm{eu}} /(\mathrm{eu}-k) \cdot \mathcal{D}(\mathbf{L})^{\mathrm{eu}}$.

Remark. The algebra $\mathcal{D}_{k}(\mathbf{L})$ is known as a ring of twisted differential operators on $\mathbb{P}^{n-1}$. In the special case when $k$ is an integer, the algebra $\mathcal{D}_{k}(\mathbf{L})$ turns out to be isomorphic to $\mathcal{D}\left(\mathbb{P}^{n-1}, \omega^{\otimes(-k)}\right)$, the algebra of global algebraic differential operators acting on the sections of the line bundle $\omega^{\otimes(-k)}$. $\diamond$

The torus $T \subset S L_{n}$, formed by diagonal matrices, has a unique open dense orbit in $\mathbb{P}^{n-1}$. Let $\mathbf{L}^{\text {reg }} \subset \mathbf{L}$ be the preimage of this $T$-orbit in $\mathbf{L}$, and let $\mathcal{D}_{k}\left(\mathbf{L}^{\text {reg }}\right):=\mathcal{D}\left(\mathbf{L}^{\text {reg }}\right)^{\text {eu }} /($ eu $k) \cdot \mathcal{D}\left(\mathbf{L}^{\text {reg }}\right)^{\text {eu }}$ be the corresponding ring of twisted differential operators.

For each $k \in \mathbb{C}$, we now define an $\mathcal{U} \mathfrak{g}$-module $V_{k}$ as follows. The tautological $S L_{n^{-}}$ action on $\mathbb{P}^{n-1}$ lifts naturally to an action on $\mathbf{L}$, that commutes with the $\mathbb{C}^{*}$-action. Differentiating the $S L_{n}$-action gives, by restriction to $\mathbf{L}^{\text {reg }}$, a Lie algebra morphism $\varrho_{k}$ : $\mathfrak{g}=\mathfrak{s l}_{n} \longrightarrow \mathbb{C}^{*}$-equivariant vector fields on $\mathbf{L}^{\text {reg }}$. This Lie algebra morphism extends to an associative algebra homomorphism: $\mathcal{U} \mathfrak{g} \rightarrow \mathcal{D}\left(\mathbf{L}^{\text {reg }}\right)^{\text {eu }}$, and we form the composite map:

$$
\varrho_{k}: \mathcal{U} \mathfrak{g} \longrightarrow \mathcal{D}\left(\mathbf{L}^{\mathrm{reg}}\right)^{\mathrm{eu}} \rightarrow \mathcal{D}\left(\mathbf{L}^{\mathrm{reg}}\right)^{\mathrm{eu}} /(\mathrm{eu}-k) \cdot \mathcal{D}\left(\mathbf{L}^{\mathrm{reg}}\right)^{\mathrm{eu}}=\mathcal{D}_{k}\left(\mathbf{L}^{\mathrm{reg}}\right) .
$$

Let $\mathcal{D}_{k}\left(\mathbf{L}^{\text {reg }}\right) \cdot \varrho_{k}(\mathfrak{h}) \subset \mathcal{D}_{k}\left(\mathbf{L}^{\text {reg }}\right)$ be the left ideal generated by the image of Cartan subalgebra $\mathfrak{h}=$ Lie $T$. Thus, $\mathcal{D}_{k}\left(\mathbf{L}^{\text {reg }}\right) / \mathcal{D}_{k}\left(\mathbf{L}^{\text {reg }}\right) \cdot \varrho_{k}(\mathfrak{h})$ is a left $\mathcal{D}_{k}\left(\mathbf{L}^{\text {reg }}\right)$-module, hence, an $\mathcal{U} \mathfrak{g}$-module via (13.1). The proof of the following result is left to the reader.

Proposition 13.2 The $\mathfrak{g}$-module $\mathcal{D}_{k}\left(\mathbf{L}^{\mathrm{reg}}\right) / \mathcal{D}_{k}\left(\mathbf{L}^{\mathrm{reg}}\right) \cdot \varrho_{k}(\mathfrak{h})$ is isomorphic to $V_{k}$.

\section{Appendix D: Small representations.}

In this appendix we will consider the special case of the radial part construction of $\S 6$ and the Dunkl-Cherednik construction in $\S 4$ in the case when $V$ is a small finite dimensional 
representation of $\mathfrak{g}$. This situation is of special interest because in the case of small representations both constructions give the same answer.

Let $\mathfrak{g}$ be a finite dimensional simple Lie algebra. Let $\varrho: \mathcal{U} \mathfrak{g} \rightarrow \operatorname{End} V$ be a finite dimensional (not necessarily irreducible) representation such that all of its weights belong to the root lattice of $\mathfrak{g}$. Then, $\varrho$ is called small if $\varrho\left(e_{\alpha}\right)^{2} v=0$, for any root vector $e_{\alpha} \in \mathfrak{g}$ and any $v \in V\langle 0\rangle$. This condition is equivalent to saying that $2 \alpha$ is not a weight of $V$, for any root $\alpha$. Note that $V=\mathfrak{g}$, the adjoint representation of any reductive Lie algebra, is small. We refer to the paper $[\mathrm{Br}]$ for more information about small representations.

For $\mathfrak{g}=\mathfrak{s l}_{n}$, one obtains a complete understanding of small representations as follows. Note that, for any finite-dimensional $\mathfrak{g}$-module $V$, the Weyl group $W$ acts naturally on $V\langle 0\rangle$. Write $\mathbb{C}^{n}$ for the tautological representation, and $\left(\mathbb{C}^{n}\right)^{\otimes n}$ for its $n$-th tensor power. We let $\mathcal{C}$ at denote the (semisimple) abelian category of finite dimensional $\mathfrak{s l}_{n^{-}}$ representations $V$ such that all simple constituents of $V$ are among the simple constituents of $\left(\mathbb{C}^{n}\right)^{\otimes n}$.

Proposition 14.1 (i) The representation $\left(\mathbb{C}^{n}\right)^{\otimes n}$, and its dual, are both small.

(ii) An irreducible $\mathfrak{s l}_{n}$-module is small if and only if it is a submodule of either $\left(\mathbb{C}^{n}\right)^{\otimes n}$, or of its dual. In particular, any object of $\mathcal{C}$ at is a small representation.

(iii) The assignment: $V \rightsquigarrow V\langle 0\rangle$ gives an equivalence between the category $\mathcal{C}$ at and the category of finite-dimensional $S_{n}$-modules. This equivalence sends $\left(\mathbb{C}^{n}\right)^{\otimes n}$ to the regular representation of $S_{n}$.

Proof. We leave the proof to the reader, and only comment on the last statement. Let $e_{1}, \ldots, e_{n}$ the standard basis in $\mathbb{C}^{n}$. It is clear that, for $V=\left(\mathbb{C}^{n}\right)^{\otimes n}$, the element $e_{i_{1}} \otimes \ldots \otimes e_{i_{n}}$ belongs to the zero weight subspace if and only if the indices $i_{1}, \ldots, i_{n}$ are pairwise distinct. Hence the space $V\langle 0\rangle$ has a basis formed by the elements $e_{\sigma(1)} \otimes$ $\ldots \otimes e_{\sigma(n)}, \sigma \in S_{n}$. Therefore we may identify $V\langle 0\rangle$ with $\mathbb{C} S_{n}$ via the correspondence: $\sigma \longleftrightarrow e_{\sigma(1)} \otimes \ldots \otimes e_{\sigma(n)}$

Recall the Harish-Chandra homomorphism $\Psi_{V}: \mathcal{D}\left(\mathfrak{g}^{\text {rs }}\right)^{\mathfrak{g}} \longrightarrow \mathcal{D}\left(\mathfrak{h}^{\text {reg }}, \operatorname{End}_{\mathbb{C}} V\langle 0\rangle\right)^{W}$, see 6.6). On the other hand, consider the rational Cherednik algebra $\mathrm{H}_{1,1}$, with parameters: $t=1$ and $c=1$. The renormalized Dunkl homomorphism $\Theta_{1,1}: \mathrm{H}_{1,1} \rightarrow \mathcal{D}\left(\mathfrak{h}^{\mathrm{reg}}, \mathbb{C} W\right)$, see above formula (4.7), restricts to a homomorphism $\Theta_{1,1}:\left(\mathrm{H}_{1,1}\right)^{W} \rightarrow \mathcal{D}\left(\mathfrak{h}^{\text {reg }}, \mathbb{C} W\right)^{W}$, where $\left(\mathrm{H}_{1,1}\right)^{W}$ stands for the centralizer of $W$ in the algebra $\mathrm{H}_{1,1}$. Further, the action of $W$ on $V\langle 0\rangle$ gives an algebra morphism $\varpi_{V}: \mathbb{C} W \rightarrow \operatorname{End}_{\mathbb{C}} V\langle 0\rangle$.

The following result explains the relevance of the notion of small representation for the study of the Harish-Chandra homomorphism, cf. also [Br, Thm.1] 
Theorem 14.2 If $t=1$ and $c=1$ then, for any small representation $V$, the following diagram commutes

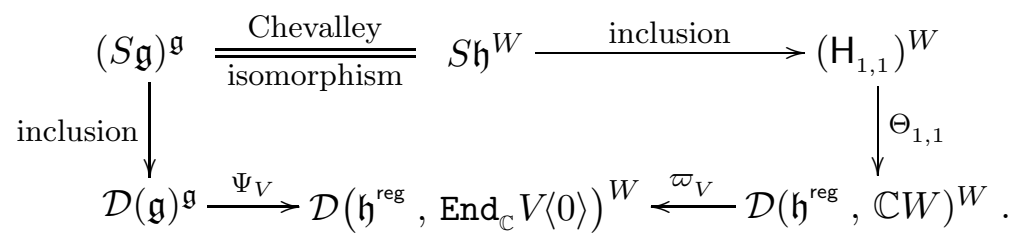

Example: Let $\varrho: \mathfrak{g} \rightarrow \operatorname{End}_{\mathbb{C}} V$ be a small representation. Then a simple calculation with 1 - and 3 -dimensional $\mathfrak{s l}_{2}$-modules shows that, for any $\alpha \in R$, the endomorphism $\varrho\left(e_{\alpha} \cdot e_{-\alpha}\right): V\langle 0\rangle \rightarrow V\langle 0\rangle$ is given by the formula: $\frac{(\alpha, \alpha)}{2} \cdot\left(\operatorname{Id}-s_{\alpha}\right)$, where $s_{\alpha}$ denotes the action on $V\langle 0\rangle$ of the simple reflection $s_{\alpha} \in W$. It follows from (6.3) that for the Harish-Chandra homomorphism $\Psi_{V}: \mathcal{D}(\mathfrak{g})^{\mathfrak{g}} \rightarrow \mathcal{D}\left(\mathfrak{h}^{\text {reg }}, \operatorname{End}_{\mathbb{C}} V\langle 0\rangle\right)^{W}$, associated to $V$, one obtains:

$$
\Psi_{V}\left(\Delta_{\mathfrak{g}}\right)=\Delta_{\mathfrak{h}}-\sum_{\alpha \in R} \frac{(\alpha, \alpha)}{2} \cdot \frac{\left(\mathrm{Id}-s_{\alpha}\right)}{\alpha^{2}}
$$

Proof of Theorem 14.2: Let $\Delta \in S \mathfrak{h}^{W} \subset \mathrm{H}_{\kappa}$ be the quadratic $W$-invariant corresponding to the Laplacian $\Delta_{\mathfrak{h}}$ on $\mathfrak{h}$. In the special case $t=1$ and $c=1$, one finds:

$$
\Theta_{1,1}(\Delta)=\Delta_{\mathfrak{h}}-\sum_{\alpha \in R} \frac{(\alpha, \alpha)}{2} \cdot \frac{\left(1-s_{\alpha}\right)}{\alpha^{2}} \in \mathcal{D}\left(\mathfrak{h}^{\text {reg }}, \mathbb{C} W\right)^{W} .
$$

Since $\Delta_{\mathfrak{g}} \in(S \mathfrak{g})^{\mathfrak{g}}$ goes under the Chevalley isomorphism to $\Delta \in S \mathfrak{h}^{W}$, comparing formulas (14.4) and (14.3) yields an equality: $\varpi_{V} \circ \Theta_{1,1}\left(\Delta_{\mathfrak{h}}\right)=\Psi_{V}\left(\Delta_{\mathfrak{g}}\right)$. Write $D$ for this element of $\mathcal{D}\left(\mathfrak{h}^{\text {reg }}, \operatorname{End}_{\mathbb{C}} V\langle 0\rangle\right)^{W}$.

Now, fix $f \in(S \mathfrak{h})^{W}$, a homogeneous element of degree $m$. First, view $f$ as an element of $(S \mathfrak{g})^{\mathfrak{g}}$, via the Chevalley isomorphism, write $f_{\mathfrak{g}}$ for the corresponding adg-invariant constant coefficient differential operator on $\mathfrak{g}$, and set: $D_{1}=\Psi_{V}\left(f_{\mathfrak{g}}\right)$. Next, view $f$ as an element of $\left(\mathrm{H}_{1,1}\right)^{W}$, and set: $D_{2}=\varpi_{V} \circ \Theta_{1,1}(f)$. The algebra $(S \mathfrak{h})^{W}$, being commutative and all the maps in the diagram of the Theorem being algebra homomorphisms, we conclude that both $D_{1}$ and $D_{2}$ commute with $D$. Further, $D_{1}, D_{2}$ are differential operators of order $\leq m$, whose principal symbols are both equal to $f$. Moreover, it is clear that $D_{1}, D_{2}$ both have homogeneity degree $-m$ with respect to the dilation action on $\mathfrak{h}^{\text {reg }}$. Thus, $D_{1}-D_{2}$ is a differential operator on $\mathfrak{h}^{\text {reg }}$ of order $\leq m-1$, that commutes with $D$ and has homogeneity degree $-m$. The proof of the Theorem is now completed by the following result.

Proposition 14.5 Let $M$ be $a \operatorname{End}_{\mathbb{C}} V\langle 0\rangle$-valued differential operator on an open subset of $\mathfrak{h}$ that commutes with $D$, has order $\leq m-1$, and homogeneity degree $-m$. Then $M=0$.

The remainder of the section is devoted to the proof of Proposition 14.5. We need an easy lemma from classical representation theory. Write $E: p \mapsto E(p)=|p|^{2}$ for the quadratic polynomial on $\mathfrak{h}^{*}$ corresponding to $\Delta \in S^{2} \mathfrak{h}$, and view it as a function on $\mathfrak{h} \oplus \mathfrak{h}^{*}$ constant along the first factor. 
Lemma 14.6 Let $S:(x, p) \mapsto S(x, p)$ be a rational function on $\mathfrak{h} \oplus \mathfrak{h}^{*}$, which is polynomial in $p \in \mathfrak{h}^{*}$. Suppose that the Poisson bracket $\{E, S\}$ equals to zero. Then $S \in \mathbb{C}\left[\mathfrak{h} \oplus \mathfrak{h}^{*}\right]$.

Proof of Lemma. We may assume without loss of generality that the function $S$ is homogeneous in the $p$-variable, say of degree $N$. By shifting $x$, we may assume further that $S$ is regular at $x=0$. Let $S=\sum_{k=0}^{\infty} S_{k}$ be the Taylor expansion of $S$ at the point $(0,0) \in \mathfrak{h} \oplus \mathfrak{h}^{*}$. Thus, $S_{k}$ is a homogeneous polynomial on $\mathfrak{h} \oplus \mathfrak{h}^{*}$ of total degree $k$. Clearly, $S_{k}$ has degree $N$ in the $p$-variable. Further, separating homogeneous components in the equation $\{E, S\}=0$ yields: $\left\{E, S_{k}\right\}=0$, for any $k \geq 0$.

The quadratic functions: $E(x, p)=|p|^{2}, H(x, p)=\langle x, p\rangle$, and $F(x, p)=|x|^{2},(x, p) \in$ $\mathfrak{h}^{\text {reg }} \times \mathfrak{h}^{*}$, are well-known to form an $\mathfrak{s l}_{2}$-triple: $\{E, H, F\}$, with respect to the Poisson bracket on $\mathbb{C}\left[\mathfrak{h} \oplus \mathfrak{h}^{*}\right]$. Hence, taking the brackets with these functions gives, for each $m \geq 0$, an $\mathfrak{s l}_{2}$-action on $\mathbb{C}\left[\mathfrak{h} \oplus \mathfrak{h}^{*}\right]^{(m)}$, the space of degree $m$ homogeneous polynomials. By representation theory of $\mathfrak{s l}_{2}$, any vector annihilated by $E$ (i.e. a highest weight vector) has to have a nonnegative weight. This means that the $p$-degree of such a polynomial is greater than or equal to its $x$-degree. In particular, the $x$-degree of $S_{k}$ is $\leq N$, which means that its total degree is $\leq 2 N$. Therefore, for $k>2 N$, we have $S_{k}=0$.

Proof of Proposition 14.5: Assume the contrary. Let $S:(x, p) \mapsto S(x, p)$ be the principal symbol of $M$, where $(x, p) \in \mathfrak{h} \oplus \mathfrak{h}^{*}$. Then $S$ is a polynomial in $p$ and $\{E, S\}=0$. We know that $S$ has homogeneity degree $-m$, in the grading where: $\operatorname{deg}(x)=1=$ $-\operatorname{deg}(p)$. Furthermore, $S$ has order $\leq m-1$ with respect to $p$. This implies that $S$ cannot be a polynomial in $x$. That contradicts the lemma, and we are done.

\section{Deformations, Poisson brackets and cohomology}

Deformations and Poisson structures. Let $\mathcal{A}$ be a flat formal (not necessarily commutative) deformation of an associative commutative algebra $A$, in other words, we are given a topologically free $\mathbb{C}[[t]]$-algebra $\mathcal{A}$ such that $\mathcal{A} / t \mathcal{A}=A$. Then, there is a canonical Poisson bracket on $A$ defined as follows. First, for each $\widetilde{u}, \widetilde{v} \in \mathcal{A}$ we have, since $A$ is commutative: $[\widetilde{u}, \widetilde{v}] \in t \mathcal{A}$, where $[\widetilde{u}, \widetilde{v}]:=\widetilde{u} \widetilde{v}-\widetilde{v} \widetilde{u}$. Let $m(\widetilde{u}, \widetilde{v}) \geq 1$ be the greatest integer (possibly $=\infty$ ) such that $[\widetilde{u}, \widetilde{v}] \in t^{m(\widetilde{u}, \widetilde{v})} \mathcal{A}$. Let $\mathbf{m} \geq 1$ be the minimum of the integers $m(\widetilde{u}, \widetilde{v})$ over all pairs $\widetilde{u}, \widetilde{v}$ as above. If $\mathbf{m}=\infty$ we set the Poisson bracket on $A$ to be zero. If $\mathbf{m}$ is finite then, given $u, v \in A$, choose any representatives $\widetilde{u}, \widetilde{v} \in \mathcal{A}$, so that $u=\widetilde{u} \bmod (t \mathcal{A})$ and $v=\widetilde{v} \bmod (t \mathcal{A})$, and put $\{u, v\}=\left(t^{-\mathbf{m}}[\widetilde{u}, \widetilde{v}]\right) \bmod (t \mathcal{A})$. It is known that the assignment: $u, v \mapsto\{u, v\}$ gives rise to a well-defined Poisson bracket on $A$, independent of the choices involved.

Observe that the equality: $\mathbf{m}=\infty$ in the construction above implies $[\widetilde{u}, \widetilde{v}] \in t^{\ell} \cdot \mathcal{A}$, $\forall \widetilde{u}, \widetilde{v} \in \mathcal{A}$, and $\forall \ell \geq 1$. Since $\bigcap_{\ell \geq 1} t^{\ell} \mathcal{A}=0$, we see that the vanishing of the Poisson bracket on $A$ forces the whole deformation to be commutative, i.e., we have

Lemma 15.1 If $\mathbf{m}=\infty$, then $\mathcal{A}$ is a commutative algebra. 
Remark. As has been observed by Hayashi [Ha], a slight modification of the above construction still applies in the case of a non-commutative algebra $A$, and gives a Poisson bracket on $\mathrm{Z}(A)$, the center of $A$. We note that, as opposed to the commutative case considered above, the Hayashi construction only works if $\mathbf{m}=1$, in particular, the bracket on $\mathrm{Z}(A)$ may turn out to be identically zero. This is so, for instance, if $\mathrm{Z}(A)$ is a specialization of the family $\left\{\mathrm{Z}\left(A_{t}\right)\right\}_{t \neq 0} . \quad \diamond$

From now until the end of this section we keep the following setup. Let $A$ be a commutative associative $\mathbb{C}$-algebra, and $\mathcal{A}$ a complete topological $\mathbb{C}[[t]]$-algebra such that $\mathcal{A} / t \cdot \mathcal{A}=A$. Write $\mathcal{K}=\mathbb{C}((t))$ for the field of formal Laurent series, and set: $\mathcal{A}_{\mathcal{K}}=$ $\mathcal{K} \widehat{\otimes}_{\mathbb{C}[t]]} \mathcal{A}$, a $\mathcal{K}$-algebra obtained by localizing at $t$ (here ' $\widehat{\otimes}$ ' stands for the completed tensor product). Let $H H^{\bullet}\left(\mathcal{A}_{\mathcal{K}}\right)$ denote the Hochschild cohomology of $\mathcal{A}_{\mathcal{K}}$ (relative to the ground field $\mathcal{K})$. Further, let $H^{\bullet}(\operatorname{Spec} A$ ) denote the ordinary singular cohomology (with complex coefficients) of $\operatorname{Spec} A$ viewed as a topological space. For any vector space $E$, write $E[[t]]$ for the space of formal power series with coefficients in $E$.

The deformation $\mathcal{A}$ gives rise to a Poisson structure $\{-,-\}$ on $A$, making $\operatorname{Spec} A$ a Poisson variety. The following result is based on the celebrated Formality theorem of Kontsevich [Kon].

Proposition 15.2 Assume that the canonical isomorphism: $\mathcal{A} / t \cdot \mathcal{A} \stackrel{\sim}{\longrightarrow} A$ can be lifted to a topological $\mathbb{C}[[t]]$-module isomorphism:

$$
\phi: \mathcal{A} \stackrel{\sim}{\longrightarrow} A[[t]] .
$$

If $\operatorname{Spec} A$ is smooth and the Poisson structure $\{-,-\}$ on $A$ is non-degenerate, i.e., makes Spec $A$ a symplectic manifold, then there is a graded $\mathcal{K}$-algebra isomorphism: $H H^{\bullet}\left(\mathcal{A}_{\mathcal{K}}\right)$ $\simeq \mathcal{K} \otimes_{\mathbb{C}} H^{\bullet}(\operatorname{Spec} A)$.

To prove the Proposition we transport the multiplication map from $\mathcal{A}$ to $A[[t]]$ via the bijection (15.3) to obtain a $\mathbb{C}[[t]]$-bilinear map $\star: A[[t]] \otimes A[[t]] \longrightarrow A[[t]],(P, Q) \mapsto$ $P \star Q=\phi\left(\phi^{-1}(P) \cdot \phi^{-1}(Q)\right)$. Expanding into powers of $t$ one can write it in the form of a star-product

$$
P \star Q=P \cdot Q+t \cdot \mu_{1}(P, Q)+t^{2} \cdot \mu_{2}(P, Q)+\ldots .
$$

The algebra $(A[[t]], \star)$ thus obtained is clearly isomorphic to $\mathcal{A}$. The following result is quite standard.

Lemma 15.5 Let $\mathcal{A}$ be a deformation of a commutative algebra A, as in Proposition 15.9. Then there exists $a \star$-product on $A[[t]]$ equivalent to (15.4), such that:

- Each map $\mu_{i}: A \times A \rightarrow A$ in this new $\star$-product is given by a certain regular bi-differential operator on $\operatorname{Spec} A$;

- $\mu_{1}(P, Q)=\{P, Q\}$, where $\{-,-\}$ stands for the Poisson bracket on $A$ induced by the deformation $\mathcal{A}$;

- The $\mathbb{C}[[t]]$-algebra $\mathcal{A}$ is isomorphic to $(A[[t]], \star)$. 
Proof of Lemma 15.5: Put $\mathcal{M}=\operatorname{Spec} A$. Then for any $k \geq 0$, the Hochschild cohomology, $H H^{k}(A)$, are well-known to be isomorphic to, $\Omega^{k}(\mathcal{M})$, the space of regular differential $k$-forms on $\mathcal{M}$. Let $C^{\bullet}(A)$ denote the standard Hochschild cochain complex, and $C_{\text {diff }}^{\bullet}(A) \subset C^{\bullet}(A)$ the subcomplex formed by poly-differential operators. Differential forms on $\mathcal{M}$ being local objects, it follows that the imbedding: $C_{\text {diff }}^{\bullet}(A) \hookrightarrow C^{\bullet}(A)$ is a quasi-isomorphism. Recall that all the terms $\mu_{i}$ in the expansion (15.4) are controlled by (second and third) Hochschild cohomology groups. Therefore, changing the star-product by an equivalent one, one may achieve that: $\mu_{i} \in C_{\text {diff }}^{2}(A)$, for all $i \geq 1$. This proves the claim about bi-differential operators. The claim concerning the Poisson bracket is well-known. The Lemma is proved.

Proof of Proposition 15.2: Set $\mathcal{M}:=\operatorname{Spec} A$, a smooth affine symplectic variety. Hence, the Poisson structure on $\mathcal{M}$ is given by a regular bi-vector field: $\beta \in \bigwedge^{2} T \mathcal{M}$. Now, fix a star-product on $A[[t]]$ as in Lemma 15.5. By (an algebraic version of) the Formality theorem of Kontsevich [Kon], this star-product is equivalent to the one obtained by the Kontsevich quantization procedure applied to an appropriate formal Poisson structure of the form: $\widehat{\beta}=\beta_{0}+t \cdot \beta_{1}+t^{2} \cdot \beta_{2}+\ldots \in\left(\bigwedge^{2} T \mathcal{M}\right)[[t]]$, such that $\beta_{0}=\beta$. Write $H P^{\bullet}(-)$, for the Poisson cohomology of a Poisson algebra, see Brylinski [Br] and references therein. Kontsevich's theorem on the cup-product on tangent cohomology, see [Kon, §8], yields a graded $\mathcal{K}$-algebra isomorphism:

$$
H H^{\bullet}\left(\mathcal{A}_{\mathcal{K}}\right) \simeq H P^{\bullet}(\mathcal{K}[\mathcal{M}], \widehat{\beta}) .
$$

Here, the RHS stands for the Poisson cohomology of the commutative $\mathcal{K}$-algebra $\mathcal{K}[\mathcal{M}]:=$ $\mathcal{K} \widehat{\otimes}_{\mathbb{C}} \mathbb{C}[\mathcal{M}]$ equipped with the $\mathcal{K}$-linear Poisson structure given by $\widehat{\beta}$.

The cohomology on the RHS of (15.6) are computed by means of $\mathcal{K} \widehat{\otimes}_{\mathbb{C}}\left(\wedge^{\bullet} T \mathcal{M}\right)$, the complex formed by poly-vector fields, with differential being induced by the Schouten bracket with the bivector $\widehat{\beta}$. It follows, since the Poisson structure $\beta=\beta_{0}$ on $\mathcal{M}$ is non-degenerate and $\mathbb{C}[[t]]$ is a local ring, that the natural pairing with $\widehat{\beta}$ induces a $\mathbb{C}[[t]]$-module isomorphism: $\left(T^{*} \mathcal{M}\right)[[t]] \stackrel{\sim}{\longrightarrow}(T \mathcal{M})[[t]]$, hence, a graded isomorphism: $\mathcal{K} \widehat{\otimes}_{\mathbb{C}}\left(\bigwedge^{\bullet} T^{*} \mathcal{M}\right) \stackrel{\sim}{\longrightarrow} \widehat{\otimes}_{\mathbb{C}}\left(\bigwedge^{\bullet} T \mathcal{M}\right)$. Brylinski showed [Br] that the de Rham differential goes under the latter isomorphism to the Poisson differential. Thus, writing $H_{D R}^{\bullet}(\mathcal{M})$ for the algebraic de Rham cohomology of $\mathcal{M}$ one obtains a natural $\mathcal{K}$-algebra isomorphism: $H P^{j}(\mathcal{K}[\mathcal{M}]) \simeq \mathcal{K} \otimes_{\mathbb{C}} H_{D R}^{\bullet}(\mathcal{M})$. But de Rham cohomology of the smooth affine variety $\mathcal{M}$ is known (Grothendieck) to be isomorphic to $H^{\bullet}(\mathcal{M})$, the ordinary singular cohomology of $\mathcal{M}$, viewed as a topological space. Combining these considerations with (15.6), we find

$$
H H^{\bullet}\left(\mathcal{A}_{\mathcal{K}}\right) \simeq H P^{\bullet}(\mathcal{K}[\mathcal{M}], \widehat{\beta}) \simeq \mathcal{K} \otimes_{\mathbb{C}} H_{D R}^{\bullet}(\mathcal{M}) \simeq \mathcal{K} \otimes_{\mathbb{C}} H^{\bullet}(\mathcal{M})
$$

In applications, Proposition 15.2 will be used in the following situation. Let $\mathcal{A}$ be a flat formal deformation of $A$, as above. Assume given an increasing filtration: $\mathbb{C}[[t]]=$ $F_{0} \mathcal{A} \subset F_{1} \mathcal{A} \subset \ldots$, on $\mathcal{A}$ such that: $\cup_{i \geq 0} F_{i} \mathcal{A}=\mathcal{A}$, and $F_{i} \mathcal{A} \cdot F_{j} \mathcal{A} \subset F_{i+j} \mathcal{A}, \quad \forall i, j \geq 0$. The filtration on $\mathcal{A}$ induces a filtration on $A=\mathcal{A} / t \cdot \mathcal{A}$, and we write $\operatorname{gr} \mathcal{A}$, resp. $\operatorname{gr} A$, for the corresponding associated graded algebra. The following result is proved by a routine argument involving, for each $i \geq 0$, an arbitrary choice of complementary subspace to $F_{i} A$ in $F_{i+1} A$. 
Lemma 15.7 Assume that: (i) gr $A$ is a finitely generated (commutative) $\mathbb{C}$-algebra, in particular, $\operatorname{dim} F_{i} A<\infty$, for any $i \geq 0$.

(ii) The algebra $\mathcal{A}$ is complete, and the isomorphism: $\operatorname{gr} \mathcal{A} / t \cdot \operatorname{gr} \mathcal{A} \stackrel{\sim}{\longrightarrow} \operatorname{gr} A$ can be lifted to a graded topological $\mathbb{C}[[t]]$-algebra isomorphism: $\operatorname{gr} \mathcal{A}=(\operatorname{gr} A)[[t]]$.

Then (15.3) holds.

Given an algebra $A$ and an idempotent $e \in A$, we say that the pair $(A, e)$ has Morita property if the map: $A \otimes A \rightarrow A$ given by $x \otimes y \rightarrow x \cdot e \cdot y$ is surjective. A similar definition applies for a complete topologically free $\mathbb{C}[[t]]$-algebra $\mathcal{A}$ in which case the map: $\mathcal{A} \widehat{\otimes}_{\mathbb{C}[t]]} \mathcal{A} \rightarrow \mathcal{A}$ involves the completed tensor product over $\mathbb{C}[[t]]$.

Let $\mathcal{A}$ be a complete topologically free $\mathbb{C}[[t]]$-algebra, and $A=\mathcal{A} / t \cdot \mathcal{A}$.

Proposition 15.8 Let $\mathbf{e} \in \mathcal{A}$ be an idempotent in $\mathcal{A}$, and $e \in A$ its reduction modulo $t$. If $(A, e)$ has Morita property, then so does $(\mathcal{A}, \mathbf{e})$.

Proof. This is an immediate consequence of the following general claim: Let $\mathbf{f}: \mathbf{V} \rightarrow$ $\mathbf{W}$ be a continuous homomorphism of complete topologically free C[[t]]-modules. If the induced map $f: \mathbf{V} / t \cdot \mathbf{V} \rightarrow \mathbf{W} / t \cdot \mathbf{W}$ is surjective, then $\mathbf{f}$ is surjective as well.

To prove the Claim, we can assume that $\mathbf{V}=V[[t]], \mathbf{W}=W[[t]]$, and $\mathbf{f}=\sum_{j \geq 0} t^{j} \cdot f_{j}$, where $f_{j}: V \rightarrow W$. Suppose we want to solve the equation $\mathbf{f}\left(\sum t^{j} \cdot v_{j}\right)=\sum t^{i} \cdot w_{i}$, where $w_{i}$ are given. Suppose we've solved it up to order $n-1$, let's solve in order $n$. We have $f_{0}\left(v_{n}\right)+$ known things $=w_{n}$. Since $f_{0}$ is surjective, we can always solve it.

\section{Some examples}

Let $\Gamma$ be a finite subgroup of $\mathrm{SL}_{2}$. Consider the wreath product $\boldsymbol{\Gamma}_{n}=S_{n} \ltimes \Gamma^{n}$, and use the notation as at the beginning of $\S 9$. We will consider the symplectic reflection algebra $\mathrm{H}_{0, k, c}\left(\boldsymbol{\Gamma}_{n}\right)$, for generic $k, c$. Our first result in this section is

Theorem 16.1 Any simple $\mathrm{H}_{0, k, c}$-module restricts to the regular representation of $\boldsymbol{\Gamma}_{n}$.

This theorem and proposition 3.8 imply the following corollary:

Corollary 16.2 For wreath-products, the Calogero-Moser space $\mathrm{Spec} \mathrm{Z}_{0, k, c}$ is smooth.

Before proving the theorem, we prove the following lemma.

Lemma 16.3 Let $E$ be a finite dimensional representation of $\mathrm{H}_{0, k, c}$. Then as a $\boldsymbol{\Gamma}_{n}$-module, $E$ has the form $\operatorname{Ind}_{S_{n}}^{\boldsymbol{\Gamma}_{n}} L$, where $L$ is a unique $S_{n}$-module. 
Proof of Lemma 16.3. To show that $E$ is induced, it suffices to show that $\operatorname{tr}_{E}(g)=0$ if $g \in \boldsymbol{\Gamma}_{n}$ is an element not conjugate to an element of $S_{n}$. We may assume that $g=$ $\left(g_{1}, g_{2}, \ldots, g_{m}\right)$, where $g_{i} \in S_{n_{i}} \ltimes \Gamma^{n_{i}}$, with $\sum_{i=1}^{m} n_{i}=n$, and where $g_{1}=\sigma \cdot(\alpha, 1, \ldots, 1)$, for some cycle $\sigma$, and $\alpha \in \Gamma$, a nontrivial element.

Let $a, b \in \mathbb{C}^{2}$, and consider the commutator $[(a, 0, \ldots, 0),(b, b, \ldots, b, 0, \ldots, 0)]$ in $\mathrm{H}_{0, k, c}$ (here and below, the number of copies of $b$ in the element $(b, b, \ldots, b, 0, \ldots, 0) \in V=$ $\left(\mathbb{C}^{2}\right)^{\oplus n} \subset \mathrm{H}_{0, k, c}$ equals $n_{1}=$ length of the cycle $\left.\sigma\right)$. According to the main commutation relation, we have

$$
[(a, 0, \ldots, 0),(b, b, \ldots, b, 0, \ldots, 0)]=(a, b) \cdot \sum_{\gamma \in \Gamma \backslash 1} c_{\gamma} \cdot(\gamma, 1, \ldots, 1)+k \cdot f(a, b),
$$

where $f(a, b) \in \mathbb{C}\left[\boldsymbol{\Gamma}_{n}\right]$. Therefore,

$$
\left[\left(\sigma, g_{2}, \ldots, g_{m}\right)(a, 0, \ldots, 0),(b, b, \ldots, b, 0, \ldots, 0)\right]=(a, b) \sum_{\gamma \in \Gamma \backslash 1} c_{\gamma} \cdot\left(\sigma \cdot(\gamma, 1, \ldots, 1), g_{2}, \ldots, g_{n}\right)+k \cdot \tilde{f}(a, b),
$$

where $\tilde{f}(a, b) \in \mathbb{C}\left[\boldsymbol{\Gamma}_{n}\right]$. Taking the trace of both sides of this equation in $E$, and using the facts that $k$ and $c_{\gamma}$ are generic and that traces of group elements are algebraic integers, we get

$$
\operatorname{tr}_{E}\left(\sigma \cdot(\gamma, 1, \ldots, 1), g_{2}, \ldots, g_{n}\right)=0 .
$$

The lemma is proved.

Proof of Theorem 16.1. In the previous lemma we showed that, for any $\gamma \in \boldsymbol{\Gamma}_{n}$, the trace of $\gamma$ in $E$ vanishes unless $\gamma$ is not conjugate to an element of $S_{n} \subset \boldsymbol{\Gamma}_{n}$. Thus, it remains only to show that the trace of any nontrivial permutation $\sigma$ in $E$ vanishes.

Let us take $y=(b, b, \ldots, b)$, and $x=(0, \ldots, 0, a, 0, \ldots, 0), a, b \in \mathbb{C}^{2}$, where $a$ stands in the $i$-th place. For any permutation $\sigma$ with $m$ cycles, let us consider the element $\sigma[x, y]=[\sigma x, y]$ of $\mathrm{H}_{0, k, c}$. The trace of this element in $E$ is obviously zero. Thus, writing this trace explicitly using the main commutation relation $(1.15($ iii $)$ ), and taking into account that $k, c$ are generic, we get

$$
\sum_{\{j \mid j \neq i\}} \sum_{\gamma \in \Gamma} \operatorname{Tr}\left(\sigma \cdot s_{i j} \cdot \gamma_{i} \cdot \gamma_{j}^{-1}, E\right) \cdot \omega_{s_{i j} \cdot \gamma_{i} \cdot \gamma_{j}^{-1}}(x, y)=0
$$

The last sum consists of two parts: the terms with $m+1$ cycles and with $m-1$ cycles. The first part is the sum over $j$ belonging to the same cycle of $\sigma$ as $i$. In this sum, the element $\sigma \cdot s_{i j}$ has $i, j$ in different cycles, so the term containing the trace of $\sigma \cdot s_{i j} \cdot \gamma_{i} \cdot \gamma_{j}^{-1}$ is zero. Indeed, for $\gamma=1$ this term comes with a zero coefficient, and for $\gamma \neq 1$ the element has nontrivial cycle monodromies (= conjugacy class of the product of the elements of $\Gamma$ in a cycle), hence, it cannot be conjugated into $S_{n}$. Thus, its trace is zero by Lemma 16.3. Thus, only the second part of the sum, over $j$ belonging to the cycles of $\sigma$ other than the one that contains $i$, remains. In this case, $i$ and $j$ are in the same cycle of $\sigma \cdot s_{i j}$, so the trace is $\gamma$-independent, and after computing $\omega_{s_{i j} \cdot \gamma_{i} \cdot \gamma_{j}^{-1}}$ and simplifications we have

$$
\sum_{\{j \mid i \notin<\sigma>j\}} \operatorname{Tr}\left(\sigma \cdot s_{i j}, E\right)=0
$$


The last equality involves only permutations with a fixed number of cycles, namely $m-1$. So the terms of the sum correspond to partitions of $n$ into $m-1$ parts. In terms of partitions, the latter sum can be expressed in a very simple way. Namely, let the length of the cycle of $\sigma$ containing $i$ be $l$. Then the other cycles define a partition $\mu=\left(\mu_{1}, \mu_{2}, \ldots, \mu_{m-1}\right), \mu_{1} \geq \mu_{2} \geq \ldots$ of $n-l$. The last equation has the form

$$
\sum_{p=1}^{m-1} \operatorname{Tr}\left(\mu_{1}, \ldots, \mu_{p}+l, \ldots, \mu_{m-1}\right)=0
$$

where trace of a partition is by definition the trace in $E$ of the corresponding permutation (to view $\left(\mu_{1}, \ldots, \mu_{p}+l, \ldots, \mu_{m-1}\right)$ as a partition one may rearrange the elements involved in the decreasing order if this happens not to be so).

Now, let $\lambda$ be a partition of $n$ into $n-m-1$ parts, $\lambda=\left(\lambda_{1}, \ldots, \lambda_{m-1}\right), \lambda_{1} \geq \lambda_{2} \geq \ldots$. Let $p$ be the largest index for which $\lambda_{p} \neq 1$. Let $l=\lambda_{p}-1$, and $\mu$ be the partition $\left(\lambda_{1}, \ldots, \lambda_{p}-l=1,1,1, \ldots, 1\right)$ of $n-l$. Then the above equation for this $\mu$ allows one to express $\operatorname{Tr}(\lambda)$ through traces of partitions which are bigger with respect to the standard ordering than $\lambda$ (i.e. obtainable from $\lambda$ by increasing $\lambda_{q}$ and decreasing $\lambda_{r}$ with $q<r$ ). This implies that $\operatorname{Tr}(\lambda)=0$, as desired.

Proposition 16.4 (i) If $W$ is the Weyl group of type $\mathbf{A}$ or of type $\mathbf{B}=\mathbf{C}$, then the variety $\operatorname{Spec} \mathrm{Z}\left(\mathrm{H}_{0, c}\right)$ is smooth, for generic $c$.

(ii) If $W$ is the Weyl group of type $\mathbf{G}_{\mathbf{2}}$, then the variety $\operatorname{Spec} \mathrm{Z}\left(\mathrm{H}_{0, c}\right)$ is never smooth.

Proof. Part (i) is a special case of Corollary 16.2 with $\Gamma=\{1\}$ for type $\mathbf{A}$, and $\Gamma=\mathbb{Z} / 2 \mathbb{Z}$, for type B. To prove (ii), by Proposition $3.8(\mathrm{i})$, it suffices to produce, for Weyl group $W$ of type $\mathbf{G}_{\mathbf{2}}$, a simple $\mathrm{H}_{0, c}$-module of dimension less than $|W|$. We have $\operatorname{dim} \mathrm{C}=2$, hence, $c=\left(c_{1}, c_{2}\right)$, where $c_{1} \in \mathbb{C}$ and $c_{2} \in \mathbb{C}$ correspond to long and short roots, respectively.

Recall the structure and representation theory of $W=W\left(\mathbf{G}_{\mathbf{2}}\right)$. The group $W$ is the group of symmeries of the regular hexagon, so it contains rotations $\left\{r^{j}\right\}_{j=0, \ldots, 5}$, where $r$ is the rotation by $\pi / 3$, and reflections: $s_{0}, \ldots, s_{5}$, where $s_{j}$ is the reflection with respect to the line making angle $\pi j / 6$ with the $x$-axis. The element $r^{3}$ (the central symmetry) is central in $W$, and $W=\mathbb{Z} / 2 \times S_{3}$, where $\mathbb{Z} / 2=\left\{1, \mathrm{r}^{3}\right\}$, and $S_{3}=\left\{1, \mathrm{r}^{2}, \mathrm{r}^{4}, s_{0}, s_{2}, s_{4}\right\}$.

Therefore, the group $W$ has six irreducible representations: $T_{+}, T_{-}, S_{+}, S_{-}, D_{+}, D_{-}$, where $T, S, D$ are the trivial, sign, and 2-dimensional representations of $S_{3}$, and \pm denotes the action of $r^{3}$. In particular, $\mathfrak{h}=D_{-}$, and the sign representation of $W$ is $S_{+}$. We note that $|W|=12$, and consider the following two reducible 6-dimensional representations $E_{+}, E_{-}$of $W: E_{+}=T_{+} \oplus S_{+} \oplus 2 D_{-}$, and $E_{-}=T_{-} \oplus S_{-} \oplus 2 D_{+}$. Clearly, $E_{+} \oplus E_{-}=\mathbb{C} W$.

The proof of part (ii) of Proposition 16.4 is now completed by the following result.

Proposition 16.5 Let $W$ be of type $\mathbf{G}_{\mathbf{2}}$ and assume $c \in \mathrm{C}$ is generic enough. Then

(i) Any finite dimensional representation of $\mathrm{H}_{0, c}$ is a linear combination of $E_{+}$and $E_{-}$ as a $W$-module.

(ii) Each of the $W$-modules $E_{+}$and $E_{-}$admits a unique extension to an $\mathrm{H}_{0, c}$-module; in particular, $E_{+}, E_{-}$are $\mathrm{H}_{0, c}$-modules of dimension $\frac{1}{2}|W|$. 
Proof. We first prove (i). Let $E$ be any finite dimensional $\mathrm{H}_{0, c}$-module. It is clear from $W$-invariance of the symplectic form $\omega$ on $\mathfrak{h}^{*} \oplus \mathfrak{h}$, that the forms $\sum_{j=0}^{2} \omega_{s_{2 j}}(x, y)$, and $\sum_{j=0}^{2} \omega_{s_{2 j+1}}(x, y)$ on $\mathfrak{h}^{*} \oplus \mathfrak{h}$ are both nonzero multiples of the standard pairing. Taking the trace in the $\mathrm{H}_{0, c}$-module $E$ of both sides of the main commutation relation (1.15iii) for the algebra $\mathrm{H}_{0, c}$, we get:

$$
[x, y]=c_{1} \cdot \sum_{j=0}^{2} \omega_{s_{2 j}}(x, y) \cdot s_{2 j}+c_{2} \cdot \sum_{j=0}^{2} \omega_{s_{2 j+1}}(x, y) \cdot s_{2 j+1} .
$$

Recall that $c_{1}, c_{2}$ are generic, all the $s_{2 j}$ are conjugate, and all the $s_{2 j+1}$ are conjugate. Thus, we get $\operatorname{Tr}\left(s_{i}, E\right)=0$. Next, we consider the commutator $\left[s_{0} \cdot x, y\left(e_{1}\right)\right]$, where $y\left(e_{1}\right) \in \mathfrak{h}$ corresponds to the vector $e_{1}=(1,0) \in \mathbb{C}^{2}$. Then $\omega_{s_{0}}\left(x, e_{1}\right)=0$, and we get

$$
\begin{aligned}
{\left[s_{0} \cdot x, e_{1}\right]=s_{0} \cdot\left[x, e_{1}\right] } & =c_{1} \cdot \sum_{j=1}^{2} \omega_{s_{2 j}}\left(x, e_{1}\right) \cdot s_{0} \cdot s_{2 j}+c_{2} \cdot \sum_{j=0}^{2} \omega_{s_{2 j+1}}\left(x, e_{1}\right) \cdot s_{0} \cdot s_{2 j+1} \\
& =c_{1} \cdot \sum_{j=1}^{2} \omega_{s_{2 j}}\left(x, e_{1}\right) \cdot r^{2 j}+c_{2} \cdot \sum_{j=0}^{2} \omega_{s_{2 j+1}}\left(x, e_{1}\right) \cdot r^{2 j+1} .
\end{aligned}
$$

Let us now take the trace in $E$ of both sides. Recall that $r^{2}$ is conjugate to $r^{4}$ and $r$ to $r^{5}$. Thus, using again that $c_{1}, c_{2}$ are generic, we get

$$
\operatorname{Tr}\left(\mathrm{r}^{2}, E\right)=0 \quad, \quad \operatorname{Tr}\left(\mathrm{r}^{3}, E\right)+\frac{1}{2} \operatorname{Tr}(\mathrm{r}, E)=0 .
$$

It is easy to check that these equations are equivalent to saying that $E=n_{+} E_{+} \oplus n_{-} E_{-}$. Statement (i) is proved.

Now we prove (ii), and introduce the structure of an $\mathrm{H}_{0, c}$-module on $E_{+}$. We have $E_{+}=T_{+} \oplus S_{+} \oplus L \otimes D_{-}$, where $L$ is a 2-dimensional space. An action of $\mathrm{H}_{0, c}$ is defined by $W$-equivariant linear maps $f_{1}: \mathfrak{h} \rightarrow \operatorname{End}\left(E_{+}\right)$and $f_{2}: \mathfrak{h}^{*} \rightarrow \operatorname{End}\left(E_{+}\right)$.

Let $I: \mathfrak{h} \rightarrow \mathfrak{h}$ be the operator of rotation by $\pi / 2$. Identifying $\mathfrak{h}$ and $\mathfrak{h}^{*}$ with $D_{-}$, and taking into account the $W$-equivariance of $f_{j}$, we find that

$$
f_{j}(v)=\left(\begin{array}{ccc}
0 & 0 & a_{j} \otimes(v, *) \\
0 & 0 & b_{j} \otimes(I v, *) \\
\alpha_{j} \otimes v & \beta_{j} \otimes I v & 0
\end{array}\right),
$$

where $a_{j}, b_{j} \in L^{*}, \alpha_{j}, \beta_{j} \in L$. Now consider the relation $\left[f_{j}(v), f_{j}(w)\right]=0$. It is easy to calculate that this relation is equivalent to the conditions $a_{j} \otimes \alpha_{j}+b_{j} \otimes \beta_{j}=0$, and $\left(a_{j}, \beta_{j}\right)=\left(b_{j}, \alpha_{j}\right)=0$. It is not hard to show that $\alpha_{j}, \beta_{j}, a_{j}, b_{j}$ must be nonzero. Thus, by changing scale in $S_{+}$we can make sure that $b_{j}=\sqrt{-1} \cdot a_{j}, \beta_{j}=\sqrt{-1} \cdot \alpha_{j}$, and we have: $\left(a_{j}, \alpha_{j}\right)=0$.

Next consider the main relation for $\left[f_{1}(v), f_{2}(w)\right]$. After a straightforward calculation, this relation turns out to be equivalent to an additional requirement that $a_{1} \alpha_{2}+a_{2} \alpha_{1}=0$, and $a_{1} \alpha_{2}-a_{2} \alpha_{1}=k$ (where $k$ is a constant multiple of $c_{1}+c_{2}$ ). There is obviously a unique solution to these equations, up to linear transformations of $L$ : namely, $a_{1}, a_{2}$ is the dual basis to $\left\{2 k^{-1} \alpha_{2},-2 k^{-1} \alpha_{1}\right\}$.

The case of $E_{-}$is treated similarly. In this case, $c_{1}+c_{2}$ is replaced with $c_{1}-c_{2}$. Part (ii) is proved. 
Remarks (i) It is easy to check that in $E_{ \pm}$the right hand side of the main relation depends only on $c_{1} \pm c_{2}$ since the sums which are multiplied by $c_{1}, c_{2}$ are the same in $E_{+}$ and differ by sign in $E_{-}$. Therefore, if $\mathrm{f}_{+}, \mathrm{f}_{-} \in \mathbb{C} W$ are the idempotents corresponding to $E_{+}, E_{-}$then one has: $\mathbf{f}_{ \pm} \cdot \mathrm{H}_{0, c_{1}, c_{2}}\left(\mathbf{G}_{\mathbf{2}}\right) \cdot \mathbf{f}_{ \pm}=\mathbb{C}[\mathbb{Z} / 2] \ltimes\left(\mathbf{f}_{ \pm} \cdot \mathrm{H}_{0, c_{1} \pm c_{2}}\left(\mathbf{A}_{\mathbf{2}}\right) \cdot \mathbf{f}_{ \pm}\right)$. Thus in effect we are taking a 6-dimensional representation of $\mathrm{H}_{0, l}\left(\mathbf{A}_{\mathbf{2}}\right)$ (regular as $S_{3}$-module, as it must be), which is compatible with the action of the central element of $W$. The claim we proved is that such a module exists and is unique.

(ii) Similar result holds more generally for $W=W_{n}$ being the Coxeter group of the symmetries of a regular $n$-gon, whenever $n \geq 5$ (with the $\mathbf{G}_{\mathbf{2}}$-case corresponding to $n=6$ ). Specifically, the algebra $\mathrm{H}_{0, c}\left(W_{n}\right)$ always has a simple module whose restriction to $\mathbb{C} W_{n}$ is isomorphic to a direct sum of the trivial representation, the sign representation, and of two copies of the 2-dimensional reflection-representation. Thus, $\mathrm{H}_{0, c}\left(W_{n}\right)$ has a 6-dimensional module, hence the variety $\operatorname{Spec} Z_{0, c}\left(W_{n}\right)$ is not smooth, if $n \geq 5$.

(iii) Note that our result on singularity of $\operatorname{Spec} \mathbf{Z}_{0, c}\left(\mathbf{G}_{\mathbf{2}}\right)$ agrees perfectly with a similar result for the $\mathbf{G}_{\mathbf{2}}$-type Hilbert scheme, obtained in [Ka2].

\section{Open questions}

Throughout this section $(V, \omega)$ is a finite dimensional symplectic vector space over $\mathbb{C}$, and $\Gamma \subset S p(V)$ is a finite group generated by symplectic reflections and, moreover, the triple $(V, \omega, \Gamma)$ is assumed to be indecomposable. We will identify $V^{*}$ with $V$ without further notice. Recall that $\mathrm{Z}_{0, c}:=\mathrm{Z}\left(\mathrm{H}_{0, c}\right)$. We have, cf. $\S 16$ :

Question 17.1 For which groups $\Gamma$ is the algebraic variety $\operatorname{Spec} \mathrm{Z}_{0, c}$ smooth, at least for generic values of $c \in \mathrm{C}$ ?

Recall that if $\operatorname{Spec} Z_{0, c}$ is smooth then the Poisson structure on $Z_{0, c}$ is induced by a symplectic form, by Lemma 3.5(ii).

Question 17.2 Assume that $c \in \mathrm{C}$ is such that $\mathrm{Spec} \mathrm{Z}_{0, c}$ is smooth. Is there a natural hyper-Kähler structure on $\mathrm{Spec} \mathrm{Z}_{0, c}$ compatible with the holomorphic symplectic 2-form ?

Question 17.3 For which finite groups $\Gamma \subset S p(V)$ the dimensions of the Poisson cohomology groups of the Poisson algebra $\mathbb{C}[V]^{\Gamma}$ are given by: $\operatorname{dim} H P^{2 i}\left(\mathbb{C}[V]^{\Gamma}\right)=\mathbf{n}(i)$, and $\operatorname{dim} H P^{\text {odd }}\left(\mathbb{C}[V]^{\Gamma}\right)=0$ ?

An argument analogous to our proof of Theorem 2.16 yields the following

Proposition 17.4 If the answer to question 17.3 is affirmative for $\Gamma$, then the family $\left\{Z_{0, c}\right\}_{c \in C}$ gives a universal deformation of $(S V)^{\Gamma}$ in the class of Poisson algebras.

Problem 17.5 Study representation theory of the algebra $\mathrm{H}_{t, c}$. Specifically, describe the set of those $(t, c) \in \mathbb{C} \oplus \mathrm{C}$ such that:

(i) The algebra $\mathrm{H}_{t, c}$ is not simple, and/or has modules whose Gelfand-Kirillov dimension is less than $\frac{1}{2} \operatorname{dim} V$.

(ii) The algebra $\mathrm{H}_{t, c}$ has finite-dimensional modules. 
For rational Cherednik algebras, problem 17.5(ii) has been studied by Cherednik (see e.g. $[\mathrm{ChM}]$ for the $\mathfrak{s l}_{2}$-case).

Let $\mathrm{Q}$ denote the skew field of fractions of the Weyl algebra $\mathrm{A}_{1}(V)$. The group $\Gamma$ acts naturally on $Q$ by algebra automorphisms. We have the following analogue of the Gelfand-Kirillov conjecture

Conjecture 17.6 For any $(t, c) \in \mathbb{C}^{*} \oplus \mathrm{C}$, there is an isomorphism between $\mathrm{Q} \# \Gamma$ and the Goldie ring of fractions of the algebra $\mathrm{H}_{t, c}$, which is identical on the subalgebra $\mathbb{C} \Gamma$.

This would imply the following result

Proposition 17.6* If Conjecture 17.6 holds then, for any $(t, c) \in \mathbb{C}^{*} \oplus \mathrm{C}$, the skew field of fractions of the algebra $\mathbf{e H}_{t, c} \mathbf{e}$ is isomorphic to $\mathrm{Q}^{\Gamma}$.

A 'quasi-classical' analogue of Proposition $17.6^{*}$ is

Conjecture 17.7 For any $c \in \mathrm{C}$, the variety $\operatorname{Spec} \mathrm{Z}_{0, c}$ is birationally isomorphic to $V / \Gamma$ as a Poisson variety.

We remark that the existence of a (faithful) Dunkl-type representation, see Proposition 4.11, for the rational Cherednik algebra $\mathrm{H}_{0, c}$ associated with any complex reflection group $\Gamma$ yields (without assuming Conjecture 17.6):

Proposition 17.7* Proposition 17.6* and Conjecture 17.7 hold true in the case where $\Gamma$ is a complex reflection group in $\mathfrak{h}$, and $V=\mathfrak{h} \oplus \mathfrak{h}^{*}$.

Question 17.8 Does the isomorphism: $H^{\bullet}\left(\mathrm{H}_{t, c}\right) \simeq \mathrm{gr}_{\bullet}^{F}(\mathrm{Z \Gamma})$ of Theorem 1.d(i) hold for all $(t, c) \in \mathbb{C}^{*} \times \mathrm{C}$ without exception?

Problem 17.9 Prove that the equality in Theorem 7.⿹ (ii) holds for all $k \in \mathbb{C}$ without exception.

Recall the setup of $\S 5$, esp. Definition 5.9. Let $P \in \mathbb{C}\langle x, y\rangle$ be a noncommutative polynomial in two variables. Call $P$ central if, for any $n$, the element $\sum_{i} P\left(x_{i}, y_{i}\right)$ is central in $\mathrm{H}_{0, c}$, where the algebra $\mathrm{H}_{0, c}$ is associated with $\mathfrak{g}=\mathfrak{g l}_{n}$.

Question 17.10 Find all central polynomials.

The above arguments show that the space of central polynomials is an $\mathbb{L}$-submodule of $\mathbb{C}\langle x, y\rangle$ which contains $x^{k}$ and $y^{k}$ for all $k$. So denoting by $E$ the $\mathbb{L}$-module generated by $x^{k}$ and $y^{k}$, we might ask if any central polynomial belongs to $E$. In particular, any element of $E$ is palindromic (i.e. invariant under the antiinvolution of $\mathbb{C}\langle x, y\rangle$ which fixes $x$ and $y$ ), so one might ask whether every central polynomial is palindromic.

Recall that according to Proposition 1.10, we have a canonical isomorphism: $K(\Gamma)$ $\simeq K\left(\operatorname{Spec} \mathrm{Z}_{0, c}\right)$. Further, Theorem 1.8 says: $H^{\bullet}\left(\operatorname{Spec} \mathrm{Z}_{0, c}\right) \simeq \mathrm{gr}_{\bullet}(\mathrm{Z} \Gamma)$. Therefore, the Chern character map $c h: \mathbb{C} \otimes_{\mathbb{Z}} K\left(\operatorname{Spec} Z_{0, c}\right) \stackrel{\sim}{\longrightarrow} H^{\bullet}\left(\operatorname{Spec} Z_{0, c}\right)$ may be viewed as an isomorphism $c h: \mathbb{C} \otimes_{\mathbb{Z}} K(\Gamma) \stackrel{\sim}{\longrightarrow} \operatorname{gr} F(Z \Gamma)$. On the other hand, identifying Z $\Gamma$ with the 
space of class functions on $\Gamma$, and associating to any $\Gamma$-module $E$ its character $\operatorname{Tr}_{E}$, gives a vector space isomorphism: $\mathbb{C} \otimes_{\mathbb{Z}} K(\Gamma) \stackrel{\sim}{\longrightarrow} \mathrm{Z} \Gamma, E \mapsto \mathrm{Tr}_{E}$.

Problem 17.11 Compute the composite map ch。 $\operatorname{Tr}^{-1}: \mathrm{Z} \Gamma \stackrel{\sim}{\longrightarrow} \mathbb{C} \otimes_{\mathbb{Z}} K(\Gamma) \rightarrow \operatorname{gr}_{\bullet}^{F}(\mathrm{Z} \Gamma)$.

Let $\mathrm{H}_{0, c}$ be the Cherednik algebra associated to a root system $R$ in $\mathfrak{h}^{*}$. Recall the finite map $\Upsilon: \operatorname{Spec} Z_{0, c} \longrightarrow \mathfrak{h}^{*} / W \times \mathfrak{h} / W$, see Proposition 4.16. Let $\chi \in \Upsilon^{-1}(0)$ be a point in the zero-fiber, viewed as a maximal ideal $\chi \subset \mathrm{Z}_{0, c}$, and $(\chi) \subset \mathrm{H}_{0, c}$ the two-sided ideal in $\mathrm{H}_{0, c}$ generated by $\chi$. We assume that $\operatorname{Spec} \mathrm{Z}_{0, c}$ is smooth, so that one has an algebra isomorphism: $\mathrm{H}_{0, c} /(\chi) \simeq$ End $_{\mathbb{C}} \mathbb{C} \Gamma$. It follows readily from this isomorphism that the space $\mathbf{e} \cdot\left(\mathrm{H}_{0, c} /(\chi)\right) \cdot \mathbf{e}_{-} \subset \mathrm{H}_{0, c} /(\chi)$ is 1-dimensional. Let $\varepsilon_{\chi}$ denote a base vector in $\mathbf{e} \cdot\left(\mathrm{H}_{0, c} /(\chi)\right) \cdot \mathbf{e}_{-}$.

Now, the action of two commutative subalgebras: $\mathbb{C}\left[\mathfrak{h}^{*}\right], \mathbb{C}[\mathfrak{h}] \subset \mathrm{H}_{0, c}$ generate two subspaces: $\mathbb{C}\left[\mathfrak{h}^{*}\right] \cdot \varepsilon_{\chi} \subset \mathrm{H}_{0, c} /(\chi)$, and $\varepsilon_{\chi} \cdot \mathbb{C}[\mathfrak{h}] \subset \mathrm{H}_{0, c} /(\chi)$, which are stable under left, resp. right, $\mathbb{C} W$-action on $\mathrm{H}_{0, c} /(\chi)$ by multiplication.

Conjecture 17.12 Assume $R$ is the root system of type $\mathbf{A}_{\mathbf{n}-\mathbf{1}}$. Then, for each $\chi \in$ $\Upsilon^{-1}(0)$ :

(i) There is a unique (up to $W$-conjugacy) pair of subsets $S_{1}, S_{2} \subset R$, such that one has $W$-module isomorphisms:

$$
\mathbb{C}\left[\mathfrak{h}^{*}\right] \cdot \varepsilon_{\chi} \simeq \operatorname{Ind}_{W\left(S_{1}\right)}^{W} \mathbf{1} \quad, \quad \varepsilon_{\chi} \cdot \mathbb{C}[\mathfrak{h}] \simeq \operatorname{Ind}_{W\left(S_{2}\right)}^{W} \operatorname{sign},
$$

where $W\left(S_{i}\right) \subset W$ denotes the Weyl subgroup generated by $S_{i}$. Moreover, each of the sets $S_{i}$ (but not $S_{1}$ and $S_{2}$ simultaneously) is a subset of a basis of simple roots in $R$.

(ii) The two $W$-modules in (i) have a single non-zero irreducible constituent, $L_{\chi} \in$ Irrep $(W)$, in common (see $[\mathrm{Gi}, 4.16,4.17])$.

Further, for $\mathfrak{g}=\mathfrak{s l}_{n}$, let $\phi:$ Spec $Z_{0, c} \stackrel{\sim}{\longrightarrow} \mathcal{M}_{n}$ be the isomorphism of Spec $Z_{0, c}$ with the Calogero-Moser space. Let $\mathbb{O}_{\chi} \subset \mathfrak{s l}_{n} \times \mathfrak{s l}_{n}$ be the $\mathrm{PGL}_{n}$-conjugacy class corresponding to a point $\chi \in \operatorname{Spec} Z_{0, c}$ under that isomorphism. Set $\mathbb{O}_{\chi}^{(i)}, i=1,2$, for the image of $\mathbb{O}_{\chi}$ under the corresponding projection $\mathrm{pr}_{i}: \mathfrak{s l}_{n} \times \mathfrak{s l}_{n} \rightarrow \mathfrak{s l}_{n}$. It is clear that, for any $\chi \in \Upsilon^{-1}(0)$, the set $\mathbb{O}_{\chi}^{(i)}$ is a nilpotent conjugacy class in $\mathfrak{s l}_{n}$.

Conjecture 17.13 The simple $W$-module $L_{\chi}$ introduced in Conjecture 17.12 corresponds to the nilpotent orbit $\mathbb{O}_{\chi}^{(1)} \subset \mathfrak{s l}_{n}$ via the Springer correspondence. Similarly, the module sign $\otimes L_{\chi}$ corresponds to the nilpotent orbit $\mathbb{O}_{\chi}^{(2)} \subset \mathfrak{s l}_{n}$ via the Springer correspondence.

Conjecture 17.14 The scheme-theoretic multiplicity of $\Upsilon^{-1}(0)$ at the point $\chi$ equals $\left(\operatorname{dim} L_{\chi}\right)^{2}$.

Problem 17.15 For any Weyl group $W$, and $c \in \mathrm{C}$ such that $\mathrm{Spec} \mathrm{Z}_{0, c}$ is smooth, find a natural bijection: $\Upsilon^{-1}(0) \stackrel{\sim}{\longleftrightarrow} \operatorname{Ireps}(W), \chi \mapsto L_{\chi}$, such that the scheme-theoretic multiplicity of $\Upsilon^{-1}(0)$ at any point $\chi \in \Upsilon^{-1}(0)$ equals $\left(\operatorname{dim} L_{\chi}\right)^{2}$. 
In the setup of Theorem 11.21, we expect the following $\Gamma$-analogue of [LS1] to hold Conjecture 17.16 Let $u \in \mathcal{D}\left(\mathrm{R}_{n}\right)^{\mathrm{PG}_{\Gamma, n}}$ be a differential operator which annihilates all $\mathrm{PG}_{\Gamma, n}$-invariant rational functions on $\mathrm{R} Q_{n}$. Then $u \in\left(\mathcal{D}\left(\mathrm{R} Q_{n}\right) \cdot\left(\operatorname{ad}-\chi_{c^{\prime}}\right)\left(\mathfrak{p g}_{\Gamma, n}\right)\right)^{\mathrm{PG}_{\Gamma, n}}$.

\section{Appendix G: Results of Kostant and shift operators}

The notion of shift operator is quite important in the theory of Calogero-Moser systems. The goal of this Appendix is to place this notion in the framework of Lie theory.

Fix $\mathfrak{g}$, a semisimple Lie algebra with a triangular decomposition $\mathfrak{g}=\mathfrak{n}_{-} \oplus \mathfrak{h} \oplus \mathfrak{n}$. Let $\operatorname{pr}_{\mathfrak{h}}: S \mathfrak{g} \rightarrow S \mathfrak{g} / S \mathfrak{g} \cdot\left(\mathfrak{n}+\mathfrak{n}_{-}\right)=S \mathfrak{h}$ be the corresponding projection. Set $\mathfrak{b}=\mathfrak{h}+\mathfrak{n}$, the fixed Borel subalgebra in $\mathfrak{g}$. Write $\rho$ for the half-sum of positive roots, and $\check{\delta} \in S \mathfrak{h}$ for the product of positive coroots. Let $L_{\mu}$ denote the irreducible $\mathfrak{g}$-module with highest weight $\mu$.

Below $\mathfrak{g}=\mathfrak{s l}_{n}$. Let $\mathrm{H}_{1, k}$ be the rational Cherednik algebra associated with the root system of $\mathfrak{s l}_{n}$. We view the family $\left\{\mathrm{H}_{1, k}\right\}_{k \in \mathbb{C}}$ as a single flat $\mathbb{C}[k]$-algebra. Also, we view $\check{\delta}$ as an element $\check{\delta}=\delta_{y} \in S \mathfrak{h} \subset \mathrm{H}_{1, k}$.

Recall the symmetriser idempotent $\mathbf{e}$ and the "anti-symmetriser idempotent": $\mathbf{e}_{-}=$ $\sum_{w \in W}(-1)^{\ell(w)} \cdot w$. The Dunkl representation $\Theta_{k}: \mathrm{H}_{k} \rightarrow\left(\mathcal{D}\left(\mathfrak{h}^{\text {reg }}\right) \# W\right)[k]$, see $\S 4$, restricts to a map

$$
\Theta_{k}: \mathbf{e}_{-} \cdot \mathrm{H}_{k} \cdot \mathbf{e} \longrightarrow \mathbf{e}_{-} \cdot\left(\mathcal{D}\left(\mathfrak{h}^{\text {reg }}\right) \# W\right) \cdot \mathbf{e} \otimes_{\mathbb{C}} \mathbb{C}[k] \simeq \mathcal{D}\left(\mathfrak{h}^{\text {reg }}\right)^{\text {sign }}[k],
$$

where $\mathcal{D}\left(\mathfrak{h}^{\text {reg }}\right)^{\text {sign }}$ stands for the subspace of $\mathcal{D}\left(\mathfrak{h}^{\text {reg }}\right)$ formed by the $W$-anti-symmetric differential operators. The operator $\mathbf{S}:=\Theta_{k}\left(\mathbf{e}_{-} \cdot \delta_{y} \cdot \mathbf{e}\right) \in \mathcal{D}\left(\mathfrak{h}^{\text {reg }}\right)^{\text {sign }}[k]$ is called the shift operator (this definition differs from the original one, and is due to Heckman). It is easy to check that $\mathrm{S}$ is a differential operator of order $m=$ number of positive roots, with the principal symbol $\sigma_{m}(\mathrm{~S})=\check{\delta}$. Furthermore, it is known from work of Opdam [O] that, for $\mathfrak{g}=\mathfrak{s l}_{n}$, we have: $\mathrm{S} \circ \mathrm{L}_{k}=\mathrm{L}_{k+1} \circ \mathrm{S}$. Moreover, there exists an isomorphism $\tau_{k}: \mathcal{C}_{k} \stackrel{\sim}{\longrightarrow} \mathcal{C}_{k+1}$ such that for all $L \in \mathcal{C}_{k}$ one has $\mathrm{S} \circ L=\tau_{k}(L) \circ \mathrm{S}$.

Example. For $n=2$, we have: $\mathrm{S}=\partial-\frac{k+1}{x}$.

Remark. In fact, Opdam considers the trigonometric Calogero-Moser system, while we are considering its rational degeneration. Let us point out that the results of Opdam are valid in the rational limit, while our construction below easily generalizes to the trigonometric case by replacing the Lie algebra with the corresponding group.

We are going to give a Lie theoretic construction of the shift operator.

Write $\mathbb{C}^{\circ}\left[x_{1}^{ \pm 1}, \ldots, x_{n}^{ \pm 1}\right] \subset \mathbb{C}\left[x_{1}^{ \pm 1}, \ldots, x_{n}^{ \pm 1}\right]$ for the subspace of Laurent polynomials of total degree zero. Recall the family $V_{k}=\left(x_{1} \cdot \ldots \cdot x_{n}\right)^{k} \cdot \mathbb{C}^{\circ}\left[x_{1}^{ \pm 1}, \ldots, x_{n}^{ \pm 1}\right], k \in \mathbb{C}$, of $\mathfrak{s l}_{n}$-representations, see the beginning of $\S 7$. Let $V$ be the nonzero finite dimensional subrepresentation of the representation $V_{1}$, i.e., $V=S^{n}\left(\mathbb{C}^{n}\right)=$ degree $n$ polynomials. The natural 'multiplication' map:

$$
f \otimes\left(x_{1} \cdot \ldots \cdot x_{n}\right)^{k} \cdot P \mapsto\left(x_{1} \cdot \ldots \cdot x_{n}\right)^{k} \cdot(f P)=\left(x_{1} \cdot \ldots \cdot x_{n}\right)^{k+1} \cdot\left(\frac{f}{x_{1} \cdot \ldots \cdot x_{n}} P\right)
$$


gives a $\mathfrak{g}$-module homomorphism: $V \otimes V_{k} \rightarrow V_{k+1}$. This morphism induces a $\mathfrak{g}$-equivariant pairing $\nu: \mathcal{D}\left(\mathfrak{g}^{\mathrm{rs}}, V\right)^{\mathfrak{g}} \otimes \mathbb{C}\left[\mathfrak{g}^{\mathrm{rs}}, V_{k}\right]^{\mathfrak{g}} \longrightarrow \mathbb{C}\left[\mathfrak{g}^{\mathrm{rs}}, V_{k+1}\right]^{\mathfrak{g}}$. Let $\left\{v_{i}\right\}$ be a basis of $V$, and $\left\{v^{i}\right\}$ the dual basis of $V^{*}$. Recall that since $V_{k}\langle 0\rangle$ is 1-dimensional, the restriction to $\mathfrak{h}^{\text {reg }}$ gives an isomorphism: $\mathbb{C}\left[\mathfrak{g}^{\text {rs }}, V_{k}\right]^{\mathfrak{g}} \stackrel{\sim}{\longrightarrow} \mathbb{C}\left[\mathfrak{h}^{\text {reg }}, V_{k}\langle 0\rangle\right]^{W}=\mathbb{C}\left[\mathfrak{h}^{\text {reg }}\right]^{W}$.

View elements of $S \mathfrak{g}$ as constant coefficient differential operators on $\mathfrak{g}$.

Theorem 18.1 (i) For $\mathfrak{g}=\mathfrak{s l}_{n}$, there exists a $\mathfrak{g}$-module embedding $j: V^{*} \hookrightarrow S^{n(n-1) / 2} \mathfrak{g}$, such that $\operatorname{pr}_{\mathfrak{h}}\left(j\left(V^{*}\right)\right)$ is the line in $S \mathfrak{h}$ spanned by $\check{\delta}$.

(ii) The assignment: $f \mapsto \sum j\left(v^{i}\right) f \otimes v_{i}$ defines a $V$-valued invariant differential operator $\widehat{S} \in \mathcal{D}(\mathfrak{g}, V)^{\mathfrak{g}}=(\mathcal{D}(\mathfrak{g}) \otimes V)^{\mathfrak{g}}$, such that, for any $\mathfrak{g}$-invariant function $f: \mathfrak{g}^{\mathrm{rs}} \rightarrow V_{k}$, one has:

$$
\left.(\nu(\widehat{\mathrm{S}} \otimes f))\right|_{\mathfrak{h}^{\text {reg }}}=\mathrm{S}\left(\left.f\right|_{\mathfrak{h}^{\text {reg }}}\right) .
$$

Before entering the proof of the Theorem, we are going to put it into a more general framework of Kostant's results. It is worth noticing that Kostant's motivation had nothing to do with the theory of Calogero-Moser systems and shift operators.

Kostant results. We keep the notation as at the beginning of this section. In particular, let $\mathfrak{g}=\mathfrak{n}_{-} \oplus \mathfrak{h} \oplus \mathfrak{n}$ be an arbitrary semisimple Lie algebra with a fixed triangular decomposition, and $\mathfrak{b}=\mathfrak{h}+\mathfrak{n}$. Set $m=$ number of positive roots, so that $\operatorname{dim} \mathfrak{g}=\mathrm{rk} \mathfrak{g}+2 m$. Write $\Lambda^{\bullet} \mathfrak{g}$ and $\Lambda^{\bullet} \mathfrak{g}^{*}$ for the exterior algebras over $\mathfrak{g}$ and $\mathfrak{g}^{*}$, respectively.

Let $A b$ denote the set parametrising all the abelian ideals $\mathfrak{a} \subset \mathfrak{b}$ having dimension rk g. For each $\mathfrak{a} \in A b$, the element $\Lambda^{\text {rk }} \mathfrak{a} \mathfrak{a} \in \Lambda^{\text {rk }} \mathfrak{g}$ is clearly an ad $\mathfrak{b}$-weight vector, hence a highest weight vector of a simple $\mathfrak{g}$-submodule in $\Lambda^{\text {rk }} \mathfrak{g} \mathfrak{g}$. The corresponding highest weight, $\mu_{\mathfrak{a}}$, equals the sum of all the positive roots whose root vectors form a basis of $\mathfrak{a}$. Further, the Hodge 'star-operator' relative to an invariant form on $\mathfrak{g}$ gives a $\mathfrak{g}$-equivariant isomorphism $\star: \Lambda^{\text {rk }} \mathfrak{g} \mathfrak{g} \stackrel{\sim}{\longrightarrow} \Lambda^{\operatorname{dim} \mathfrak{g}-\mathrm{rk} \mathfrak{g}} \mathfrak{g}=\Lambda^{2 m} \mathfrak{g}$. We let $L_{\mathfrak{a}} \subset \Lambda^{2 m} \mathfrak{g}$ denote the simple $\mathfrak{g}$-submodule corresponding to the $\mathfrak{g}$-submodule in $\Lambda^{\text {rk }} \mathfrak{g} \mathfrak{g}$ generated by $\Lambda^{\text {rk }} \mathfrak{g}_{\mathfrak{a}}$. Thus, $L_{\mathfrak{a}} \simeq L_{\mu_{\mathfrak{a}}} ;$ moreover, all the modules $L_{\mathfrak{a}}, a \in \mathrm{Ab}$, are known to be pairwise nonisomorphic.

Write $\Lambda^{\mathrm{ev}} \mathfrak{g}^{*} \subset \Lambda^{\bullet} \mathfrak{g}^{*}$ for the even part, which is a commutative algebra. Let $d: \mathfrak{g}^{*} \longrightarrow$ $\Lambda^{2} \mathfrak{g}^{*}$ denote the map dual to the commutator map: $\mathfrak{g} \wedge \mathfrak{g} \stackrel{[,]}{\longrightarrow} \mathfrak{g}$. The map $d$ can be uniquely extended to a commutative algebra homomorphism $s: S \mathfrak{g}^{*} \rightarrow \Lambda^{\mathrm{ev}} \mathfrak{g}^{*}$. Let $A(\mathfrak{g})=\bigoplus_{i} A^{2 i} \mathfrak{g}$, be the image of $s$, naturally graded by even integers.

Theorem 18.2 (侦o2], 吕o3]) (i) The maximal $i$ such that $A^{2 i} \mathfrak{g} \neq 0$ equals $m$.

(ii) $A^{2 m} \mathfrak{g}=\bigoplus_{\mathfrak{a} \in \mathrm{Ab}} L_{\mathfrak{a}}$.

(iii) A simple $\mathfrak{g}$-module occurs in $L_{\rho} \otimes L_{\rho}$ with non-zero multiplicity if and only if it occurs in $\Lambda^{\bullet} \mathfrak{g}$. Thus, $L_{\mathfrak{a}}$ occurs in $L_{\rho} \otimes L_{\rho}$ with non-zero multiplicity, for any $\mathfrak{a} \in \mathrm{Ab}$.

Example: $\mathfrak{s l}_{n}$-case. In this case $m=n(n-1) / 2$. The root vectors in any Young diagram occupying the upper right hand corner of the Borel subalgebra of the upper triangular matrices spans an ideal in the Borel subalgebra, where the Young diagram 
progresses downward and to the left of $E_{1 n}$. If there are exactly $n-1$ boxes then the ideal is abelian. Given such a Young diagram, the sum of the roots corresponding to the boxes of the diagram form the corresponding dominant weight $\mu_{\mathfrak{a}} . \diamond$

Following [Ko2], consider the map $s^{\dagger}: \Lambda^{\mathrm{ev}} \mathfrak{g} \longrightarrow S \mathfrak{g}$, transposed to $s$ (this map is related to the Amitsur-Levitski standard identity, as explained in [Ko3]). Put: $Q(\mathfrak{g})=$ $s^{\dagger}\left(\Lambda^{2 m} \mathfrak{g}\right)$. By self-duality of $A(\mathfrak{g})$, one gets a $\mathfrak{g}$-module isomorphism: $Q(\mathfrak{g}) \simeq A^{2 m}(\mathfrak{g})=$ $\bigoplus_{\mathfrak{a} \in \mathrm{Ab}} L_{\mathfrak{a}}$. From now on we will think of the $L_{\mathfrak{a}}$ 's as sitting inside $Q(\mathfrak{g})$ via this decomposition. Recall the notion of harmonic elements in $S \mathfrak{g}$ introduced in [Ko1].

Theorem 18.3 (Kostant [Ko4]) (i) Any element of $Q(\mathfrak{g}) \subset S^{m} \mathfrak{g}$ is harmonic.

(ii) For any $\mathfrak{a} \in \mathrm{Ab}$, the space $\operatorname{pr}_{\mathfrak{h}}\left(L_{\mathfrak{a}}\right) \subset S \mathfrak{h}$ is the 1-dimensional spact spanned by $\check{\delta}$.

(iii) For any $\mathfrak{a} \in \mathrm{Ab}$, and any integer $k=1,2, \ldots$, there exists a $\mathfrak{g}$-equivariant imbedding $j: L_{k \cdot \mu(a)} \hookrightarrow S^{k \cdot m} \mathfrak{g}$ such that $\operatorname{pr}_{\mathfrak{h}}\left(j\left(L_{k \cdot \mu(a)}\right)\right) \subset S^{k \cdot m} \mathfrak{h}$ is the one dimensional space spanned by $\check{\delta}^{k}$, the $k$-th power of the product of the positive roots.

Proof (after Kostant). Recall the standard Koszul differential $d$ on $\Lambda^{\bullet} \mathfrak{g}^{*}$. Note that the algebra $A(\mathfrak{g})$ is generated by exact 2 -forms, relative to $d$. Hence, any element of $A(\mathfrak{g})$ is exact. It follows that, for any ad $\mathfrak{g}$-invariant $u \in S \mathfrak{g}^{*}$, the element $s(u)$ is ad $\mathfrak{g}$-invariant and exact. But it is well-known that any non-zero ad $\mathfrak{g}$-invariant in $\Lambda^{\bullet} \mathfrak{g}^{*}$ represents a non-zero cohomology class in $H^{*}(\mathfrak{g})$, so that any exact ad $\mathfrak{g}$-invariant must be equal to zero. Thus, $s$ must vanish on the ideal $I \subset S \mathfrak{g}$ generated by the ad $\mathfrak{g}$-invariants of positive degree. Part (i) follows from this, by duality.

Let $\omega \in \Lambda^{2 m} \mathfrak{g}$ be defined as the wedge product of all the root vectors, with respect to the triangular decomposition. It is a zero weight vector which transforms according to the sign representation of the Weyl group since any simple reflection clearly takes $\omega$ to $-\omega$. Identify $\mathfrak{g}^{*}$ with $\mathfrak{g}$ and $\mathfrak{h}^{*}$ with $\mathfrak{h}$. It follows that the element $\operatorname{pr}_{\mathfrak{h}}\left(s^{\dagger}(\omega)\right)$ is an element of $S^{m} \mathfrak{h}$ that transforms, under the Weyl group action, according to the sign character of $W$. But any such element is known to be proportional to $\check{\delta}$. Thus, $\operatorname{pr}_{\mathfrak{h}}\left(s^{\dagger}(\omega)\right) \in \mathbb{C} \cdot \check{\delta}$.

To prove (ii), take the orthocomplement $D$ in $\Lambda^{2 m} \mathfrak{g}$ of the one dimensional space spanned by $\omega$. Since $s\left(x^{m}\right)$ is a non-zero multiple of $\omega$, for any $x \in \mathfrak{h}^{\text {reg }}$, it follows that $s^{\dagger}(D)$, viewed as an element of $S \mathfrak{g}^{*}$, vanishes on the elements of the form $x^{m}, x \in \mathfrak{h}^{\text {reg }}$. Hence, by the previous paragraph, $\operatorname{pr}_{\mathfrak{h}}\left(s^{\dagger}\left(\Lambda^{2 m} \mathfrak{g}\right)\right)$, is contained in the one dimensional space spanned by the product of positive coroots. But $L_{\mathfrak{a}}$ is contained in $\Lambda^{2 m} \mathfrak{g}$. Hence, for any $a \in \mathrm{Ab}$, either $\operatorname{pr}_{\mathfrak{h}}\left(s^{\dagger}\left(L_{\mathfrak{a}}\right)\right)$ vanishes or is contained in $\operatorname{pr}_{\mathfrak{h}}\left(s^{\dagger}\left(L_{\mathfrak{a}}\right)\right) \subset \mathbb{C} \cdot \check{\delta}$. We claim it cannot vanish. Indeed if $\operatorname{pr}_{\mathfrak{h}}\left(s^{\dagger}\left(L_{\mathfrak{a}}\right)\right)$ vanished then since it is stable under the Ad $G$-action it follows that $\operatorname{Ad} g\left(\operatorname{pr}_{\mathfrak{h}}\left(s^{\dagger}\left(L_{\mathfrak{a}}\right)\right)\right)$ vanishes, for all $g \in G$. Identifying $S \mathfrak{g}$ with $\mathbb{C}[\mathfrak{g}]$ via an invariant form, that would mean that elements of $\operatorname{pr}_{\mathfrak{h}}\left(s^{\dagger}\left(L_{\mathfrak{a}}\right)\right)$ vanish on all adjoint transforms of $\mathfrak{h}$. But that would imply that they vanish identically on $\mathfrak{g}$, which is impossible.

\footnotetext{
${ }^{2}$ this result has been first obtained (via a case by case argument) by Ranee Brylinski in her PhD Thesis.
} 
To prove (iii), view $L_{\mathfrak{a}}$ as a vector subspace in $S^{m} \mathfrak{g}$. Clearly, elements of the form $\left\{v^{k}, v \in L_{\mathfrak{a}} \subset S^{m} \mathfrak{g}\right\}$ span an ad $\mathfrak{g}$-stable subspace in $S^{k \cdot m} \mathfrak{g}$. Furthermore, let $v_{\mu}$ be a highest weight vector for $L_{\mathfrak{a}}$. Then $\left(v_{\mu}\right)^{k}$ is a highest weight vector, and the $\mathfrak{g}$-module generated by this vector has highest weight $k \cdot \mu_{\mathfrak{a}}$. This gives an imbedding $j_{k}: L_{k \cdot \mu_{\mathfrak{a}}} \hookrightarrow$ $S^{k \cdot m} \mathfrak{g}$. It remains to observe that, for any $v \in S^{m} \mathfrak{g}$, one has: $\operatorname{pr}_{\mathfrak{h}}\left(v^{k}\right)=\left(\operatorname{pr}_{\mathfrak{h}}(v)\right)^{k}$. It follows, that $\operatorname{pr}_{\mathfrak{h}}\left(L_{k \cdot \mu_{\mathfrak{a}}}\right)$ is contained in the linear span of $\left\{\operatorname{pr}_{\mathfrak{h}}\left(v^{k}\right), v \in L_{\mathfrak{a}}\right\}$, which has been shown to be the line spanned by $\check{\delta}^{k}$. Finally, $\operatorname{pr}_{\mathfrak{h}}\left(L_{k \cdot \mu_{\mathfrak{a}}}\right) \neq 0$ since $\operatorname{pr}_{\mathfrak{h}}\left(\left(v_{\mu}\right)^{k}\right) \neq 0$.

Fix $a \in \mathrm{Ab}$. Let $\left\{v_{i}\right\}$ be a basis of $L_{\mathfrak{a}}$, and $\left\{v^{i}\right\}$ the dual basis of $L_{\mathfrak{a}}^{*}$. Since, $Q(\mathfrak{g})$ is a self-dual module, the above results hold for $L_{\mathfrak{a}}^{*}$ as well as for $L_{\mathfrak{a}}$. In particular, we have the canonical $\mathfrak{g}$-module imbedding $j: L_{\mathfrak{a}}^{*} \hookrightarrow S^{m} \mathfrak{g}$, as a harmonic subspace. Put $S_{\mathfrak{a}}:=\sum j\left(v^{i}\right) \otimes v_{i}$. This is a $\mathfrak{g}$-invariant $L_{\mathfrak{a}}$-valued differential operator on $\mathfrak{g}$ with constant coefficients, of order $m$. Moreover, part (ii) of Theorem 18.3 insures that for $\sigma_{m}\left(S_{\mathfrak{a}}\right)$, the principal symbol of $S_{\mathfrak{a}}$, one has: $\operatorname{pr}_{\mathfrak{h}}\left(\sigma_{m}\left(S_{\mathfrak{a}}\right)\right)=\check{\delta}$.

For any integer $k \geq 0$, one has a canonical $\mathfrak{g}$-module projection: $L_{\mathfrak{a}} \otimes L_{k \cdot \mu_{\mathfrak{a}}} \rightarrow$ $L_{(k+1) \cdot \mu_{\mathfrak{a}}}$. It induces a natural pairing $\nu: \mathcal{D}\left(\mathfrak{g}^{\mathrm{rs}}, L_{\mathfrak{a}}\right)^{\mathfrak{g}} \otimes \mathbb{C}\left[\mathfrak{g}^{\mathrm{rs}}, L_{k \cdot \mu_{\mathfrak{a}}}\right]^{\mathfrak{g}} \longrightarrow \mathbb{C}\left[\mathfrak{g}^{\mathrm{rs}}, L_{(k+1) \cdot \mu_{\mathfrak{a}}}\right]^{\mathfrak{g}}$. Recall the isomorphism: $\mathbb{C}\left[\mathfrak{g}^{\text {rs }}, L_{k \cdot \mu_{\mathfrak{a}}}\right]^{\mathfrak{g}} \stackrel{\sim}{\longrightarrow} \mathbb{C}\left[\mathfrak{h}^{\text {reg }}, L_{k \cdot \mu_{\mathfrak{a}}}\langle 0\rangle\right]^{W}$, induced by restriction to $\mathfrak{h}^{\text {reg }}$. Thus, the radial part of $S_{\mathfrak{a}}$ gives a differential operator

$$
\Psi\left(S_{\mathfrak{a}}\right) \in \mathcal{D}\left(\mathfrak{h}^{\mathrm{reg}}, \operatorname{Hom}_{\mathbb{C}}\left(L_{k \cdot \mu_{\mathfrak{a}}}\langle 0\rangle, L_{(k+1) \cdot \mu_{\mathfrak{a}}}\langle 0\rangle\right)\right)^{W} .
$$

Proof of Theorem 18.1. If the Young diagram corresponding to $a \in \mathrm{Ab}$ is the top row or the last column we get $L_{\mathfrak{a}}=S^{n}\left(\mathbb{C}^{n}\right)(=V)$, or its dual. Thus, Theorem 18.2(iii),(vi) yields a canonical imbedding $j: V \hookrightarrow S^{n(n-1) / 2} \mathfrak{g}$. Part (i) of Theorem 18.1 now follows from Theorem 18.3(ii).

Further, since $V_{k}\langle 0\rangle$ is a 1-dimensional space, the operator (18.4) may be viewed in this case as a scalar valued differential operator on $\mathfrak{h}^{\text {reg }}$. The principal symbol of this differential operator equals $\check{\delta}$, by part (i) of the theorem.

Moreover, we have: $\Psi\left(S_{\mathfrak{a}}\right) \circ \mathrm{L}_{k}=\mathrm{L}_{k+1} \circ \Psi\left(S_{\mathfrak{a}}\right)$, because $S_{\mathfrak{a}}$ transforms $G$-invariant functions with values in $V_{k}$ into $G$-invariant functions with values in $V_{k+1}$.

Thus, the operator $\Psi\left(S_{\mathfrak{a}}\right)$ satisfies all the properties of the shift operator. Therefore, it must coincide with the shift operator, by the uniqueness results, due to Opdam.

Remark. An alternative proof of Theorem 18.1 can be deduced from the results of [EK]. Specifically, first it was proven in [EK] that there exists a nonzero homomorphism $\Xi: L_{\rho} \rightarrow$ $L_{\rho} \otimes V^{*}$. For any $X \in \mathfrak{g}$ the element $e^{X} \in G$ gives a well-defined automorphism of the vector space $L_{\rho}$, and one considers the composite map $\Xi_{\circ} e^{X}: L_{\rho} \stackrel{e^{X}}{\longrightarrow} L_{\rho} \stackrel{\Xi}{\longrightarrow} L_{\rho} \otimes V^{*}$ as an element of $\left(\operatorname{End}_{\mathbb{C}} L_{\rho}\right) \otimes V^{*}$. Thus, taking the trace: $\operatorname{End}_{\mathbb{C}}\left(L_{\rho}\right) \rightarrow \mathbb{C}$, on obtains a $V^{*}$-valued holomorphic function: $\left.X \mapsto \operatorname{Tr}\right|_{L_{\rho}}\left(\Xi_{\circ} e^{X}\right)$, on $\mathfrak{g}$. It has been shown in [EK] that, for any $X \in \mathfrak{h}$, one has the identity: $\left.\operatorname{Tr}\right|_{L_{\rho}}\left(\Xi \circ e^{X}\right)=\prod_{\alpha>0}\left(e^{\langle\alpha, X\rangle / 2}-e^{-\langle\alpha, X\rangle / 2}\right)=$ 
Weyl denominator, where we have identified $V^{*}\langle 0\rangle$ with $\mathbb{C}$. This implies that

$$
F:\left.\quad X \mapsto \frac{1}{\frac{n(n-1)}{2} !} \cdot \operatorname{Tr}\right|_{L_{\rho}}\left(\Xi \circ X^{n(n-1) / 2}\right) \quad, \quad X \in \mathfrak{g},
$$

is a $V^{*}$-valued function on $\mathfrak{g}$ which defines the required embedding, with property (i).

We note that this remark is in good agreement with Theorem 18.2(viii), since $L_{\rho}^{*}=L_{\rho}$.

\section{References}

[AFLS] J. Alev, M.A. Farinati, T. Lambre, and A.L. Solotar: Homologie des invariants d'une algèbre de Weyl sous l'action d'un groupe fini. J. of Algebra 232 (2000), 564-577.

[Alv] M.S. Alvarez: Algebra structure on the Hochschild cohomology of the ring of invariants of a Weyl algebra under a finite group. Preprint 1999.

[Ba] V. Batyrev: Non-Archimedean integrals and stringy Euler numbers of log-terminal pairs. J. Eur. Math. Soc. (JEMS) 1 (1999), 5-33.

[BB] A. Beilinson, J. Bernstein: A proof of Jantzen conjectures. I. M. Gelfand Seminar, 1-50, Adv. Soviet Math., 16, Part 1, Amer. Math. Soc., Providence, RI, 1993.

[BG] A. Braverman, and D. Gaitsgory: Poincaré-Birkhoff-Witt theorem for quadratic algebras of Koszul type. J. Algebra 181 (1996), 315-328.

[BBG] J. Bernstein, A. Braverman, and D. Gaitsgory: The Cohen-Macaulay property of the category of (g, K)-modules. Selecta Math.(New Ser.) 3(1997), 303-314.

[BGS] A. Beilinson, V. Ginzburg, W. Soergel: Koszul duality patterns in Representation Theory, J. Amer. Math. Soc. 9 (1996), 473-527.

[BJ] A. Braverman, A. Joseph: The minimal realization from deformation theory. J. Alg. 205 (1998), 13-36.

[Bj] J.-E. Bjork: The Auslander condition on Noetherian rings. Séminaire d'Algèbre Dubreil-Malliavin, Lecture Notes in Math., 1404 (1989), 137-173, Springer, Berlin/New York.

[BW] Yu. Berest, G. Wilson: Automorphisms and ideals of the Weyl algebra. Math. Ann. 318(2000), 127-147.

[Bl] J. Block: Cyclic homology of filtered algebras. K-Theory 1 (1987), 515-518.

[Br] A. Broer: The sum of generalized exponents and Chevalley's restriction theorem for modules of covariants. Indag. Mathem. 6 (1995), 4, 385-396.

[BMR] M. Broué, G. Malle, R. Rouquier: Complex reflection groups, braid groups, Hecke algebras. J. Reine Angew. Math. 500 (1998), 127-190.

[B] J.-L. Brylinski: A differential complex for Poisson manifolds. J. Diff. Geom. 28 (1988), 93-114.

[CG] N. Chriss, V. Ginzburg: Representation theory and complex geometry. Birkhäuser Boston, 1997.

[Ch] I. Cherednik: Double affine Hecke algebras, Knizhnik-Zamolodchikov equations, and Macdonald operators, IMRN (Duke math.J.) 9 (1992), p.171-180.

[Ch2] I. Cherednik: A unification of Knizhnik-Zamolodchikov and Dunkl operators via affine Hecke algebras. Invent. Math. 106 (1991), 411-431.

[ChM] I. Cherednik, Y. Markov: Hankel transform via double Hecke algebra, math.QA/0004116.

[Coh] A.M. Cohen: Finite quaternionic reflection groups. J. Algebra 64 (1980), 293-324.

[Co] A. Connes: Noncommutative geometry. Academic Press, Inc., 1994.

[CBH] W. Crawley-Boevey, M. Holland: Noncommutative deformations of Kleinian singularities. Duke Math. J. 92 (1998), 605-635. 
[CB] W. Crawley-Boevey: Geometry of the moment map for representations of quivers. Comp. Math. 126(2001), 257-293.

[Dr1] V. Drinfeld: On quadratic quasi-commutational relations in quasi-classical limit. Selecta Math. Sovietica. 11 (1992), 317-326.

[Dr2] V. Drinfeld: Degenerate affine Hecke algebras and Yangians. (Russian) Funktsional. Anal. i Prilozhen. 20 (1986), 69-70.

[D1] C. Dunkl: Differential-difference operators associated to reflection groups. Trans. Amer. Math. Soc. 311 (1989), 167-183.

[D2] C. Dunkl: Operators commuting with Coxeter group actions on polynomials. Invariant theory and tableaux, 107-117, IMA Vol. Math. Appl., 19, Springer, New York, 1990.

[EK] P. Etingof, A. Kirillov Jr.: Macdonald polynomials and representations of quantum groups, MRL, 1(1994), 279-296.

[EFK] P. Etingof, I. Frenkel, A. Kirillov Jr.: Spherical functions on affine Lie groups. Duke Math. J. 80 (1995), 59-90.

[E] P. Etingof: Quantum integrable systems and representations of Lie algebras. Jour. Math. Phys., 36(1995), 2636-2651.

[Gi] V. Ginzburg: Principal nilpotent pairs in a semisimple Lie algebra. I. Invent. Math. 140 (2000), 511-561.

[Gu] R. Guralnick: A note on pairs of matrices with rank 1 commutator. Lin. \& Multilin. Alg. 8(2), 97-99 (1979).

[J] A. Joseph: On a Harish-Chandra homomorphism. C.R.Acad. Sci. Paris, 324(1997), 759-764.

[Ha] T. Hayashi: Sugawara operators and Kac-Kazhdan conjecture. Invent. Math. 94 (1988), 13-52.

[HC] Harish-Chandra: Invariant differential operators and distributions on a semisimple Lie algebra. Amer. J. Math, 86 (1964), 534-564.

[He] S. Helgason: Groups and geometric analysis. Integral geometry, invariant differential operators, and spherical functions. Pure and Applied Mathematics. 113 Academic Press, 1984.

[Ho] M. Holland: Quantization of the Marsden-Weinstein reduction for extended Dynkin quivers. Ann. Sci. École Norm. Sup. 32 (1999), 813-834.

[Ka1] D. Kaledin: McKay correspondence for symplectic quotient singularities. Invent. Math. (2001), math. AG/9907087.

[Ka2] D. Kaledin: Symplectic resolutions: deformations and birational maps. math.AG/0012008

[Ki] A. Kirillov Jr.: Lectures on affine Hecke algebras and Macdonald conjectures, Bull. of the AMS, 34(1997), 251-292.

[KKS] D. Kazhdan, B. Kostant, and S. Sternberg: Hamiltonian group actions and dynamical systems of Calogero type, Comm.Pure Apll. Math., 31(1978), 481-507.

[Kon] M. Kontsevich: Deformation quantization of Poisson manifolds, I. math.QA/9709040

[Ko1] B. Kostant: Lie group representations on polynomial rings, Amer. J. Math. 85 (1963), 327-404.

[Ko2] B. Kostant: The set of abelian ideals of a Borel subalgebra, Cartan decompositions, and discrete series representations. Internat. Math. Res. Notices 5 (1998), 225-252.

[Ko3] B. Kostant: A Lie algebra generalization of the Amitsur-Levitski theorem. Adv. in Math. 40 (1981), 155-175.

[Ko4] B. Kostant: On Peterson's $2^{l}$ abelian ideals theorem, homology of loop spaces, and the Macdonald $\eta$-function formula, to appear.

[LSo] M. Lehn, C. Sorger: Symmetric groups and the cup product on the cohomology of Hilbert schemes. math.AG/0009131 (To appear in: Duke Math. Journ.).

[Le] T. Levasseur: Differential operators on a reductive Lie algebra, Lectures given at the University of Washington, Seattle, 1995. 
[LS1] T. Levasseur, J. Stafford: Invariant differential operators and a homomorphism of Harish-Chandra. J. Amer. Math. Soc. 8 (1995), 365-372.

[LS2] T. Levasseur, J. Stafford: The kernel of an homomorphism of Harish-Chandra. Ann. Sci. École Norm. Sup. 29 (1996), 385-397.

[Lu] G. Lusztig: Singularities, q-analogues, and weight multiplicities. Asterisque, 101 (1982), 208-229.

[ML] L. Makar-Limanov: On automorphisms of the free algebra with two generators. Funct. Anal. Appl. 4 (1970), 262-264.

[MR] J. C. McConnell, J. C. Robson: Noncommutative Noetherian rings. Pure and Applied Mathematics. John Wiley \& Sons, Ltd., Chichester, 1987.

[Mo] S. Montgomery: Fixed Rings of Finite Automorphism Groups of Associative Rings, LN in Math., 818 Springer-Verlag, Berlin/New-York, 1980.

[Na1] H. Nakajima: Instantons on ALE spaces, quiver varieties, and Kac-Moody algebras. Duke Math. J. 76 (1994), 365-416.

[Na2] H. Nakajima: Quiver varieties and Kac-Moody algebras. Duke Math. J. 91 (1998), 515-560.

[O] E.M. Opdam: Some applications of hypergeometric shift operators. Inv. Math., 98 (1988), 1-18.

[O2] E.M. Opdam: Lecture notes on Dunkl operators for Real and Complex reflection groups. MSJ Memoirs, Math. Soc. of Japan, 8 (2000).

[OP] M. A. Olshanetsky, A. M. Perelomov: Quantum integrable systems related to Lie algebras. Phys. Rep. 94 (1983), 313-404.

[PV] V. Popov, E. Vinberg: Invariant theory, Algebraic geometry VI, Encyclopedia of Math. Sciences, 55, pp. 123-284. Springer-Verlag, 1994.

[PP] A. Polishchuk, L. Positselsky: Quadratic Algebras. Unpublished manuscript 1996.

[Q] D. Quillen: Higher algebraic K-theory. I. Springer Lecture Notes in Math., 341 (1973), 85-147.

[Re] M. Reid: La correspondance de McKay. Séminaire Bourbaki, 867 (1999), math.AG/9911165.

[Sch] G. Schwarz: On a homomorphism of Harish-Chandra, Algebraic groups and Lie groups, 321-329, Austral. Math. Soc. Lect. Ser., 9, Cambridge Univ. Press, Cambridge, 1997.

[Se] J.-P. Serre: Algèbre locale. Multiplicites. Springer Lect. Notes in Math., 11, 1965.

[STS] M. Semenov-Tian-Shansky: Poisson Lie groups, quantum duality principle, and the quantum double. Contemp. Math., 175, 219-248. Amer. Math. Soc., Providence, RI, (1994).

[St] J. Stafford: Homological properties of the enveloping algebra $\mathcal{U}\left(\mathfrak{s l}_{2}\right)$, Math. Proc. Camb. Phil. Soc., 91(1982), 29-37.

[Va] E. Vasserot : Sur l'anneau de cohomologie du schèma de Hilbert de $\mathbf{C}^{2}$. C.R. Acad. Sci. Paris, 332 (2001), 7-12.

[VB] M. Van den Bergh: Existence theorems for dualizing complexes over non-commutative graded and filtered rings. J. Alg. 195 (1997) 662-679.

[Ve] M. Verbitsky: Holomorphic symplectic geometry and orbifold singularities. math.AG/9903175.

[VSC] A.P. Veselov, K.A. Styrkas, and O.A. Chalyh: Algebraic integrability for Schrödinger equation and finite reflection groups, Theor. and Math. physics, 94, 3, 1993.

[Wa] N. Wallach: Invariant differential operators on a reductive Lie algebra and Weyl group representations. J. Amer. Math. Soc. 6 (1993), 779-816.

[Wan] W. Wang: Hilbert schemes, wreath products, and the McKay correspondence. math.AG/9912104.

[Wat] K. Watanabe: Certain invariant subrings are Gorenstein. I, II. Osaka J. Math. 11 (1974), 1-8; 379-388.

[We] H. Weyl: Classical groups, their invariants, and representations, Princeton Univ. Press, 1949.

[Wi] G. Wilson: Collisions of Calogero-Moser particles and an adelic Grassmannian, Inv. Math. 133 (1998), 1-41. 
[YZ] A. Yekutieli, J. Zhang: Rings with Auslander dualizing complexes. J. Algebra 213 (1999), 1-51.

P.E.: Department of Mathematics, Rm 2-165, MIT, 77 Mass. Ave, Cambridge, MA 02139

etingof@math.mit.edu

V.G.: Department of Mathematics, University of Chicago, Chicago, IL 60637, USA;

ginzburg@math.uchicago.edu 